
cellular electroporation using

planar membrane models

\title{
and
}

\author{
miniaturized devices
}




\section{INVESTIGATING CELLULAR ELECTROPORATION USING PLANAR MEMBRANE MODELS AND MINIATURIZED DEVICES}

Iris van Uitert 
The research described in this thesis was carried out at the BIOS Lab-on-aChip Group of the MESA+ Institute for Nanotechnology of the University of Twente, Enschede, The Netherlands. The project was financially supported by the Physics of Fluids group from the University of Twente

Members of the committee:

Chairman

Promoter

Assistant promoter

Members
Prof.dr.ir. A.J. Mouthaan

Prof. dr. ir. A. van den Berg

Dr. ir. S. Le Gac

\author{
Prof. dr. V. Subramaniam \\ Prof. dr. ir. P.H. Veltink \\ Prof. dr. S.J Marrink \\ Dr. M.P Rols
}

Prof. T. Schmidt
University of Twente

University of Twente

University of Twente

University of Twente

University of Twente

University of Groningen

CNRS (FR)

University of Leiden

Title: Investigating cellular electroporation using planar membrane models and miniaturized devices

Author: $\quad$ Iris van Uitert

ISBN: $\quad$ 978-90-365-3093-4

Publisher: Wöhrmann Print Service, Zutphen, The Netherlands

Cover design: Wouter van den Berg and Iris van Uitert

Copyright ( 92010 by Iris van Uitert, Enschede, The Netherlands 


\section{INVESTIGATING CELLULAR ELECTROPORATION USING PLANAR MEMBRANE MODELS AND MINIATURIZED DEVICES}

PROEFSCHRIFT

ter verkrijging van

de graad van doctor aan de Universiteit Twente, op gezag van de rector magnificus, prof.dr. H. Brinksma, volgens besluit van het College voor Promoties in het openbaar te verdedigen op vrijdag 22 oktober 2010 om 15.00 uur door

Iris van Uitert

geboren op 3 april 1981

te Groningen 
Dit proefschrift is goedgekeurd door:

Promotor:

Assistant promotor:
Prof. dr. ir. A. van den Berg

Dr. ir. S. Le Gac 


\section{Table of Content}

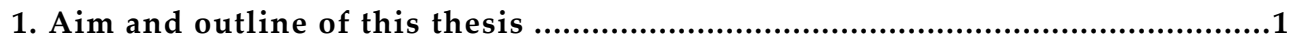

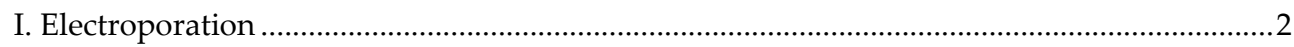

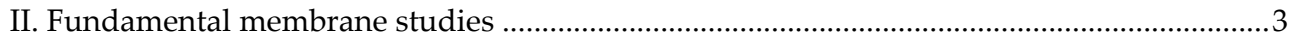

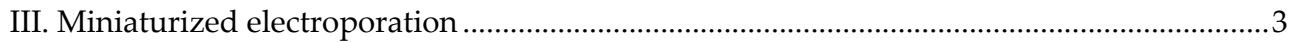

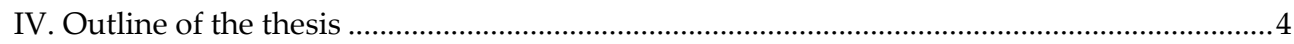

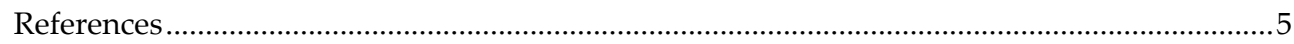

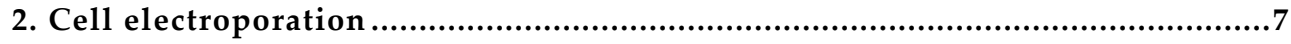

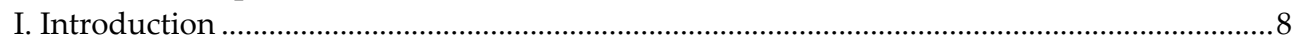

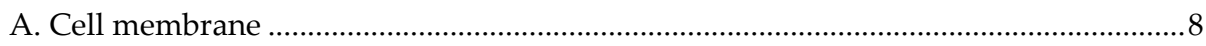

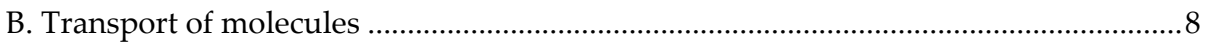

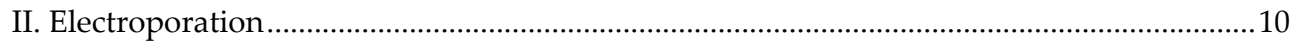

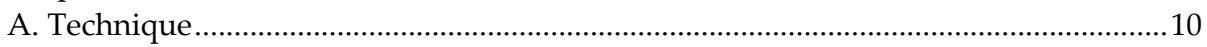

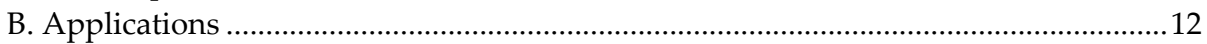

C. Theory describing the electroporation process .......................................................... 12

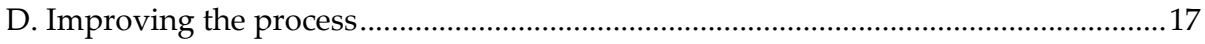

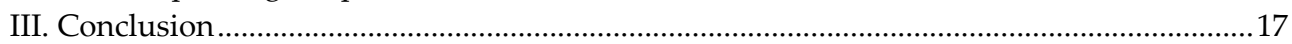

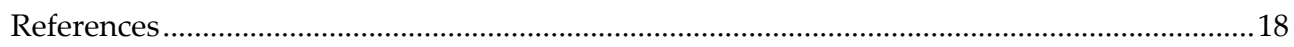

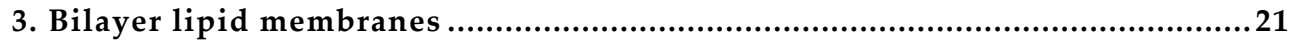

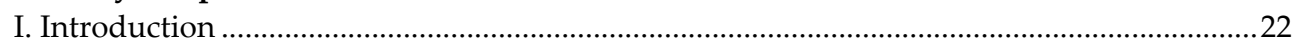

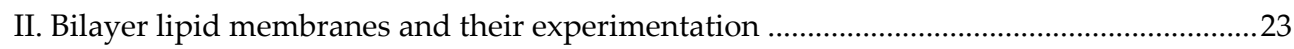

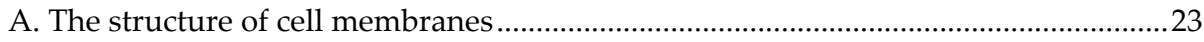

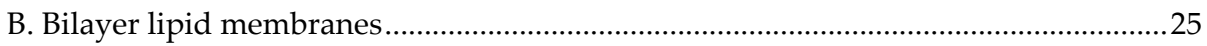

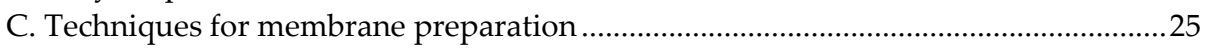

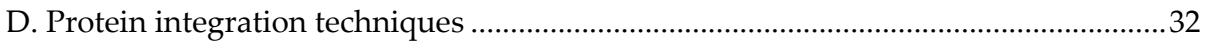

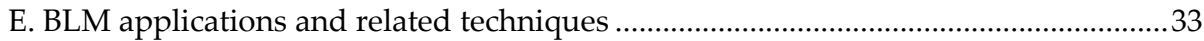

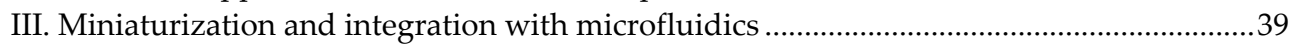

A. Motivation for the miniaturization of BLM experimentation and its integration in

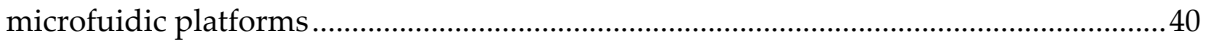

B. Different levels of miniaturization and micro-fluidic integration...............................43

IV. Microfluidics: A future standard for BLM experimentation? .............................................63

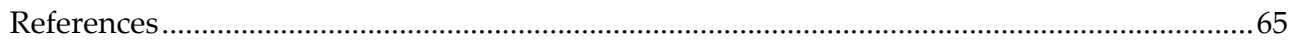

4. The influence of different membrane components on the electrical stability of

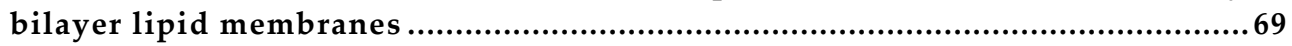

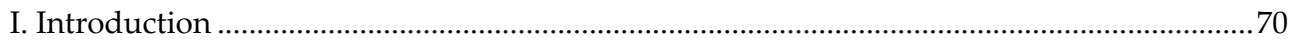

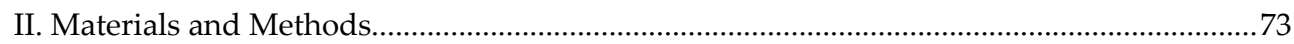

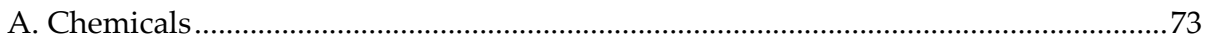

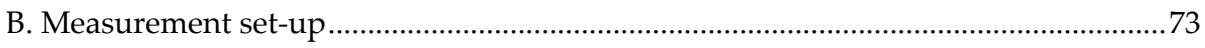

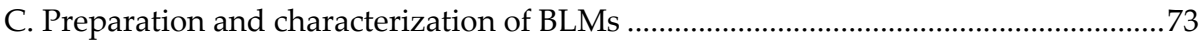

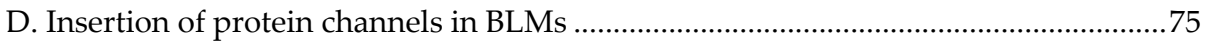

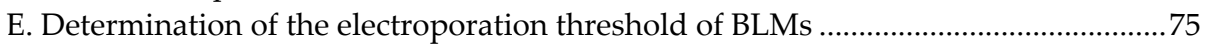

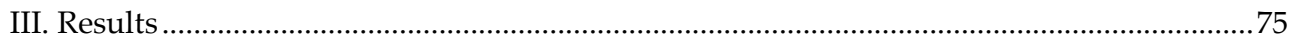

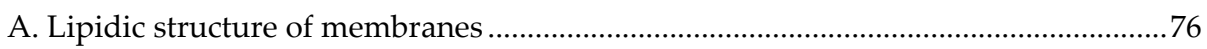




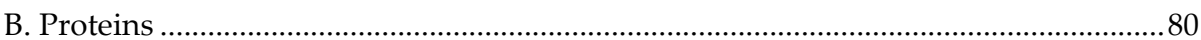

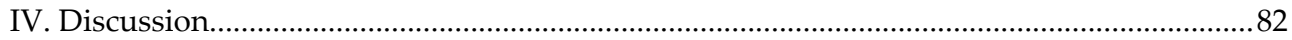

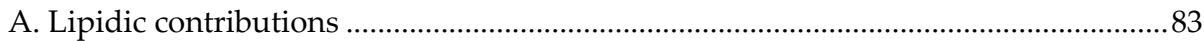

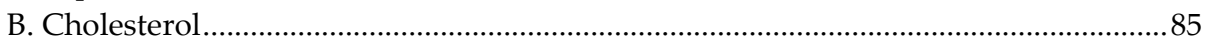

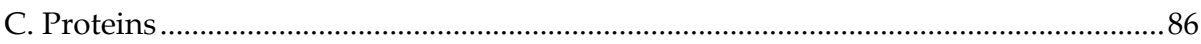

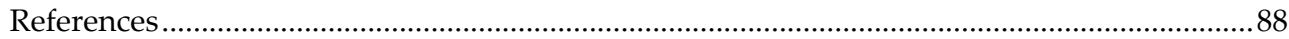

5. Determination of the electroporation onset of bilayer lipid membranes as a novel approach to establish ternary phase diagrams: example of the $L-\alpha-$

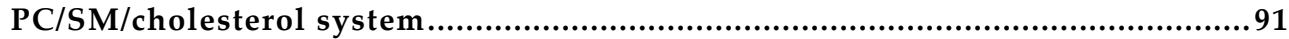

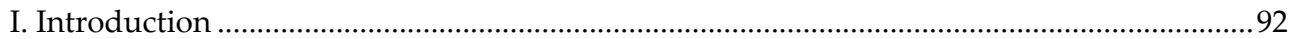

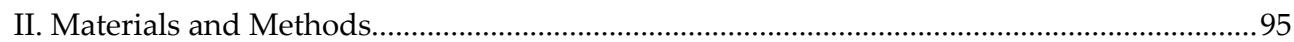

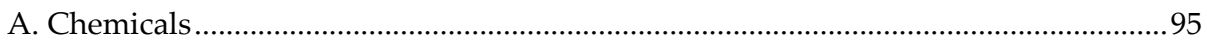

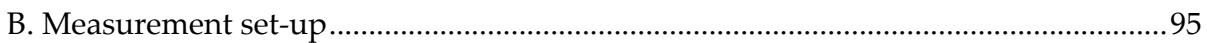

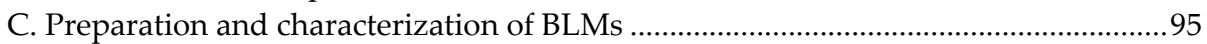

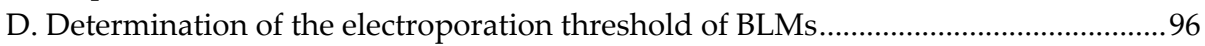

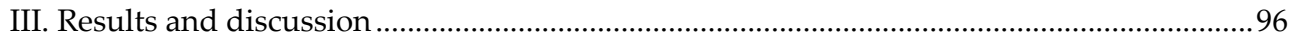

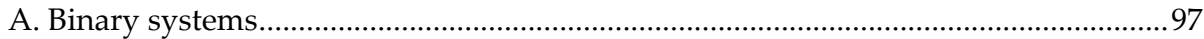

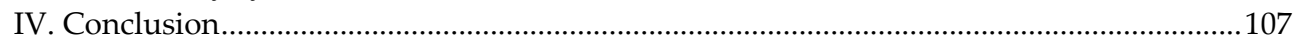

A. Electroporation threshold for complex membrane mixtures ....................................107

B. Electroporation measurements as a novel approach for establishing ternary

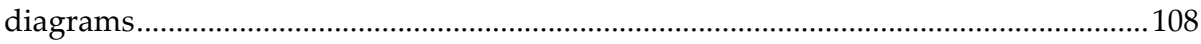

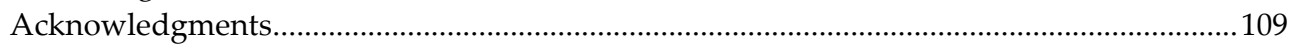

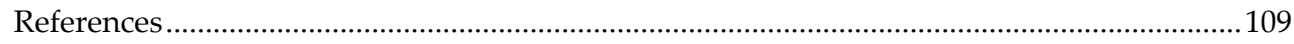

6. Cell membrane heterogeneities affect the outcome of electroporation: implications for in vivo treatment in a clinical setting ..............................113

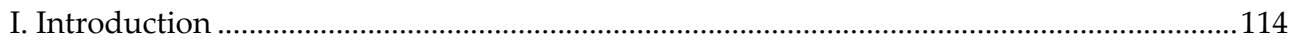

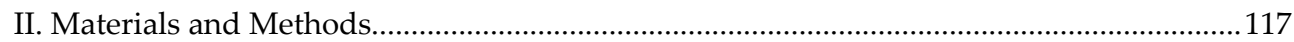

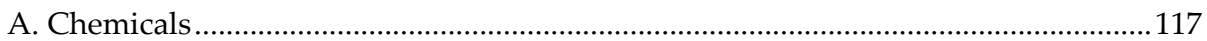

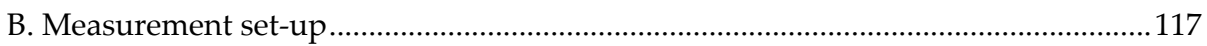

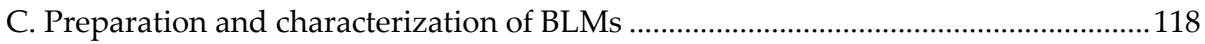

D. Determination of the electroporation threshold of BLMs........................................118

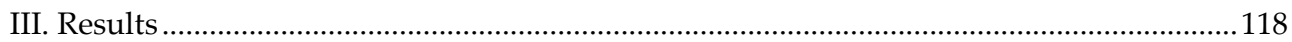

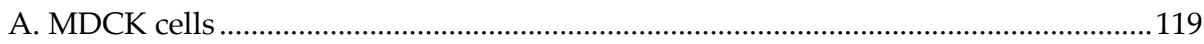

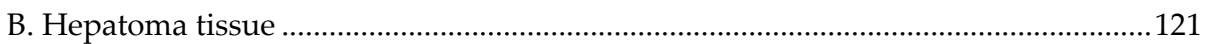

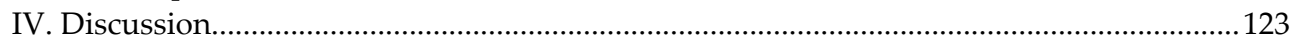

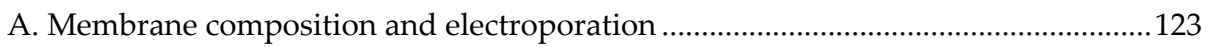

B. Membrane composition and electroporation-based treatment ..................................125

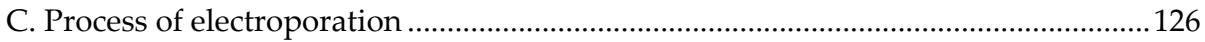

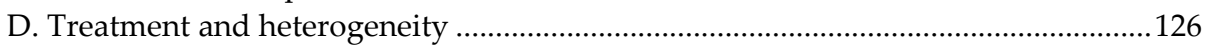

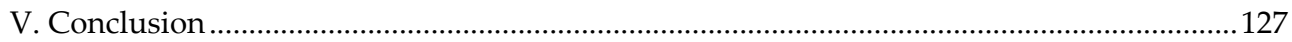

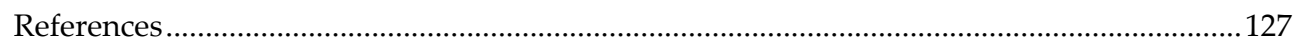

7. Bilayer lipid membranes on an integrated microfluidic platform................131

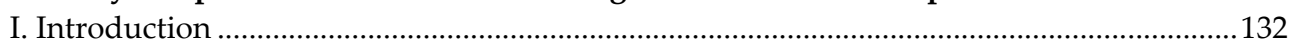

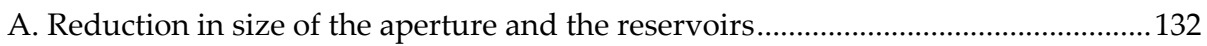




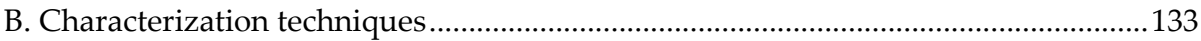

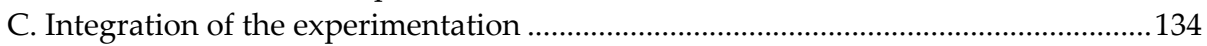

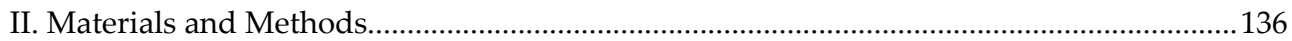

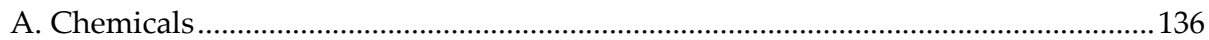

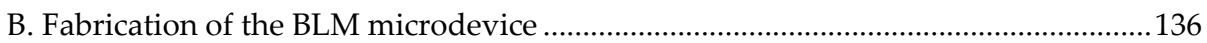

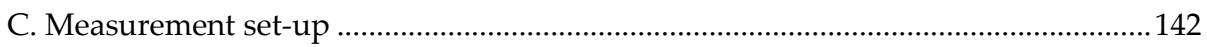

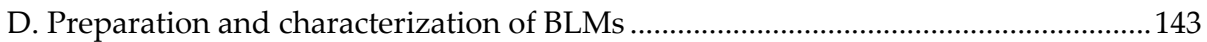

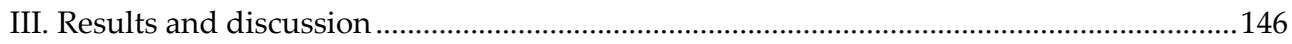

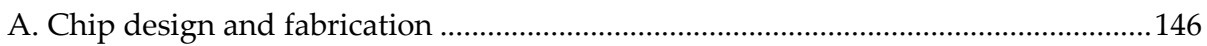

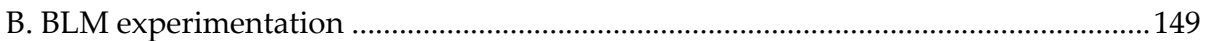

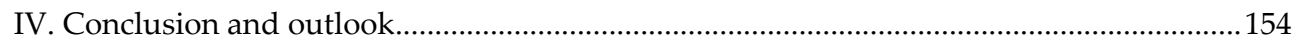

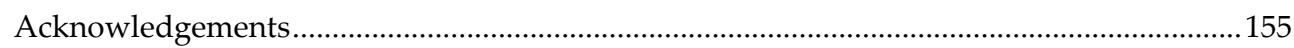

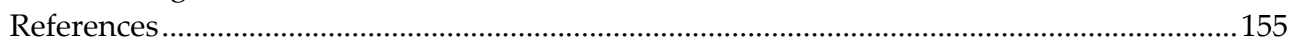

8. Miniaturized device for the electroporation of adherent (and polarized) cells

157

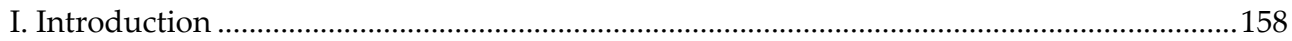

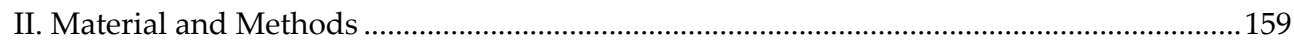

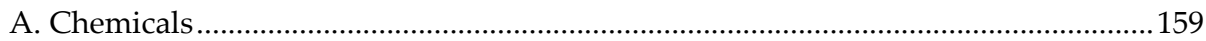

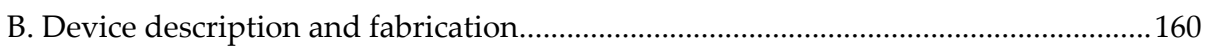

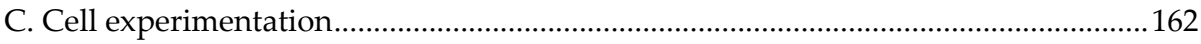

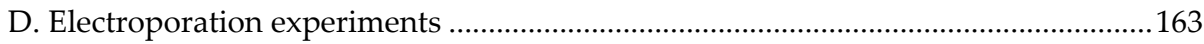

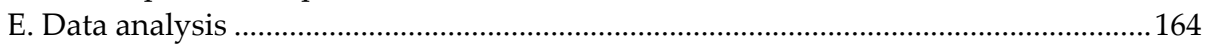

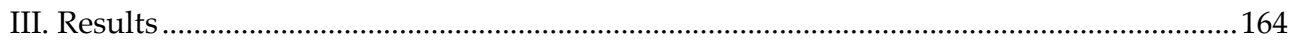

A. Characteristics of the electroporation and measurement set-up ................................164

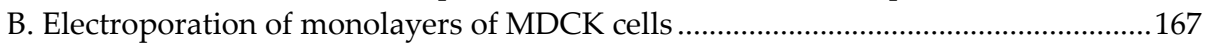

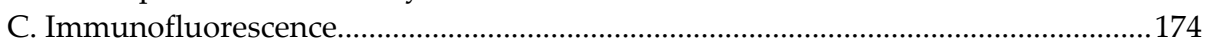

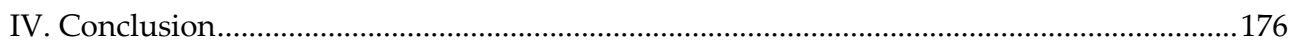

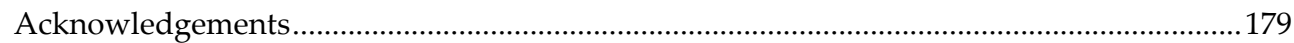

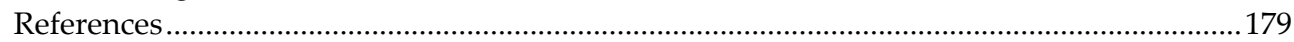

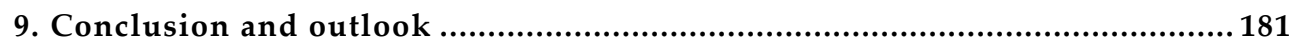

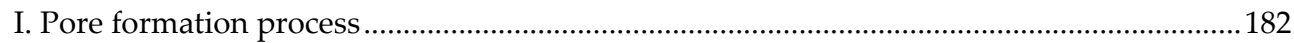

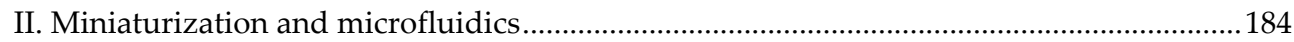

Appendix A. Review miniaturized electroporation devices .............................187

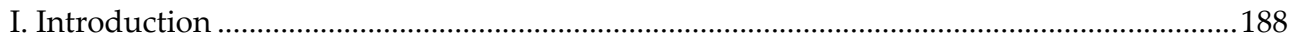

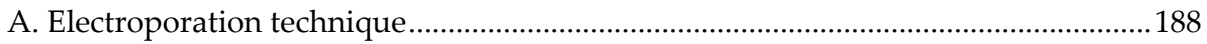

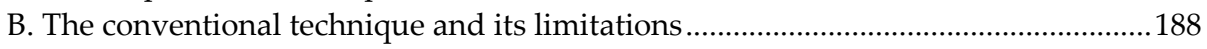

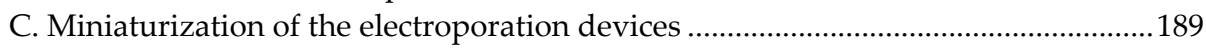

II. Miniaturized and microfluidic electroporation devices ..................................................190

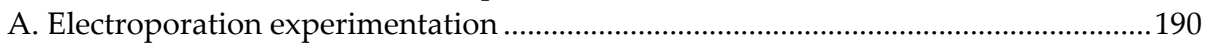

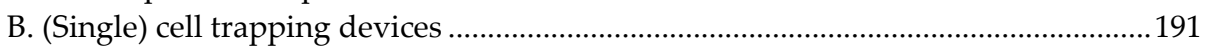

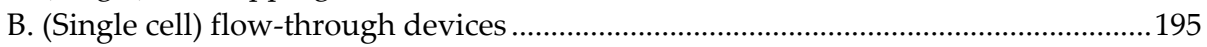

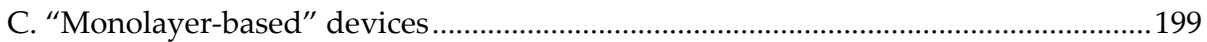

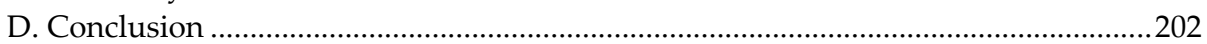

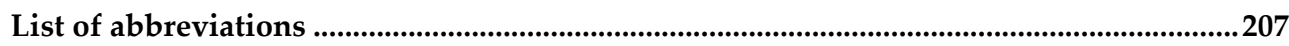




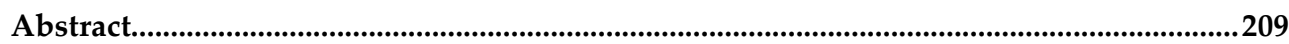

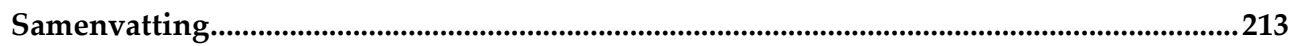

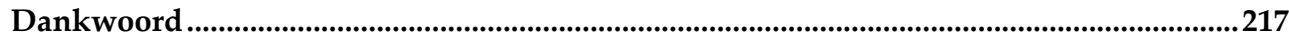

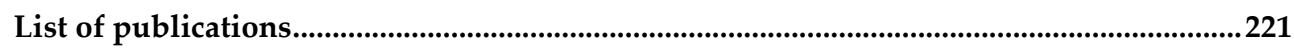




\section{Chapter 1}

\section{Aim and outline of this thesis}

The aim of this thesis, the study of the electroporation process, is briefly introduced in this chapter. The two directions taken to get a better understanding of the process and to increase the control over it are discussed. Furthermore, a brief description of the content of the different chapters is provided. 


\section{Electroporation}

A number of biotechnological and medical applications (such as drug delivery, particle delivery and gene transfection) rely on the introduction of foreign substances into a cell, and this requires a method to go through the cell membrane. Electroporation is a technique employed to transiently permeabilize cell membranes to enable access to the inside of the cell. It relies on the application of short but high electric field pulses that result in charging of the cell membrane. As soon as the transmembrane potential exceeds a certain threshold value of $0.2-1 \mathrm{~V}$, electrical breakdown of the membrane occurs, resulting in a change in the membrane conductance that indicates the formation of conducting pores. When the electric field parameters are controlled well enough, the potential across the membrane just exceeds its critical value and the jump in conductance remains transient (reversible electroporation). In this scenario, a short-lived rearrangement in the molecular structure of the membrane leads to the formation of pores and consequently to a substantial increase in the cell permeability to foreign entities [1-4]. Membrane recovery starts after termination of the pulse, allowing the cell to survive and possibly enclose any molecule added in the solution $[2,5-7]$.

Although the technique is widely employed for aforementioned applications, it still presents several limitations. Firstly, the relative success yield for viable transfection is low. This is caused both by the fact that all cells feel a different electric field depending on their properties and orientation with respect to the electric field (making the whole process difficult to control at the level of a whole cell population). Secondly, in a conventional setup, the electric field required to reach the critical potential value across the membrane is provided by a high voltage source. This not only brings along unwanted danger during experimentation, but also might results in the undesired production of harmful chemicals for the cells.

There are three different options to improve this. Firstly, by adapting the electrical parameters (for instance by decreasing the pulse width down to the ns range [8-12]), the amount of irreversible electroporated cells and the formation of unwanted chemicals at the electrode surface is reduced. Secondly, studying the fundamentals of the pore formation mechanism provides additional knowledge on the precise processes occurring in the cell membrane and consequently enables to better control the technique. Finally, miniaturization of the devices for electroporation alleviates the need for high voltage sources to generate the required voltage drop across the cell, as the distance between the electrodes is smaller. In addition to this, the electroporation process can be better controlled in miniaturized devices, since a few cells are treated, or even a single cell. This work particularly focuses on the latter two aspects of (i) getting a better understanding of pore formation, and (ii) miniaturization of the electroporation for improving the electroporation process. The motivation of this work can be found in previous research in our group, involving electroporation [13-14]. Triggered by the "still not $100 \%$ success-rate", we wanted to increase our understanding of 
the factors affecting pore formation to get a better understanding of (and hopefully control over) the electroporation process.

\section{Fundamental membrane studies}

Fundamental studies aiming at getting a better insight into the pore formation process can take two different directions: (i) theoretical and computational studies using for instance molecular dynamics simulations (MDS) [15-17] and (ii) experimental work [18-21]. In both cases, planar models are employed to simplify the situation; these models present a simpler structure and the applied electric field is homogeneous throughout the whole membrane, which is not the case for cell models and vesicles. For MDS studies, models consist of small membrane patches ( 5000 lipid molecules), whereas experimental investigations employ bilayer lipid membranes (BLMs). This thesis relies on experimental work and, therefore, the latter physical models are used. More specifically, molecular interactions in a membrane are examined to identify the key-parameters affecting the process of pore formation upon application of an electric field. This process is studied via the determination of the membrane resistance to pore formation by measuring the electroporation threshold $\left(V_{t h}\right)$ defined as the potential at which pores are detected in the membrane. Models with increasing levels of complexity are considered: bi-molecular models, ternary systems, and finally real cell membrane mimics. Finally, the BLM experimentation is implemented in a microfluidic system for enhanced experimental conditions and possible coupling of the standard electrical measurements to optical characterization of the membrane. Such a system also opens the route towards the development of multiplexed devices, which facilitates high throughput screening assays such as the development of new drugs.

\section{Miniaturized electroporation}

Devices employed for electroporating cells benefit greatly from miniaturization. These miniaturized and microfluidic devices present a wide variety of advantages compared to conventional systems. Some of those are general for all miniaturized devices such as an increase in the reproducibility of their production and experimental outcome, the presence of small-scale structures and an overall decrease of the experimentation cost. Other benefits are specific to this application (electroporation) and include the alleviation of high voltage sources, the option for the stimulation and monitoring of a few cells or even a single cell which greatly enhances the control over the process, and the possibility to shape the electric field by the addition of microstructures.

A microfluidic electroporation device for single cell electroporation has been developed in our group [13-14]. This device is capable of trapping nine cells between individual electrode pairs and to electroporate each cell with an individually optimized signal. Since the cells are trapped, their response to the applied pulses can be monitored in real time, as well as the success of gene transfection after a couple of days. Nevertheless, in the present work, adherent cells are considered and the effect of their membrane polarization on the 
process of electroporation studied. Since these cells grow as monolayers, the previously developed device is not suitable. Consequently, a novel design is developed, specifically suited for the electroporation of monolayers of adherent cells, which still enables the optical monitoring of individual cells during electroporation.

\section{Outline of the thesis}

Below an overview of the content of each of the chapters of this thesis is given.

In chapter 2 the process of electroporation is introduced and the most accepted theory for the pore formation process is presented. Besides, the limitations of the technique are discussed along with potential approaches for its improvement.

Chapter 3 introduces conventional membrane models, bilayer lipid membranes (BLMs), and evokes the current evolution in the field of BLM experimentation towards the miniaturization of the experimental set-up and its integration onto a microfluidic platform. To illustrate this, an overview of the existing miniaturized and microfluidic devices reported in the literature is given.

In chapter 4, a first series of experiments to study the process of pore formation during electroporation is described, employing the bilayer lipid membrane models (BLMs) described in chapter 3. The importance of the membrane composition on the process of electroporation is determined using binary systems of either (i) two phospholipids (glycerolipids), (ii) a phospholipid and cholesterol or (iii) a phospholipid and proteins.

In chapter 5, this study is extended to more complex models by the introduction of a third sphingolipid component; ternary systems of a glycerolipid (L- $\alpha$-PC), a sphingolipid $(\mathrm{SM})$ and cholesterol $(\mathrm{Ch})$ are employed in a similar way as in chapter 4 . The electroporation measurements reflect well the molecular interactions in the membranes and its phase composition. From these electroporation measurements, a ternary phase diagram for the L$\alpha$-PC/SM/Ch system is proposed.

In chapter 6, the complexity of the models is again increased, notably to study the influence of heterogeneities found in cell membranes (either between different cells in a tissue or in the membrane itself) on the process of membrane poration. The impact of the phospholipid composition of BLMs mimicking real cell membranes (i.e. the apical and basolateral part of MDCK cell membranes and of healthy and cancerous liver cells) is investigated on the outcome of the electroporation process. The results obtained on different cell lines are discussed as a potential methodology to derive protocols for in vivo treatment of cells with electroporation (for instance by electrochemotherapy).

In chapter 7, BLM experimentation is miniaturized and implemented in a microfluidic device. The development of the microfluidic device for dual electrical and optical measurements is described. A novel methodology for BLM preparation in a closed environment is proposed and early measurements on membrane proteins are presented.

In chapter 8, a miniaturized device for the electroporation of adherent cells is developed, where cells are grown under polarizing or not conditions. This simple device is 
subsequently applied to investigate the influence of the membrane organization of MDCK cells to their sensitivity to electroporation.

Finally, in chapter 9, the conclusions of this work are summarized. Furthermore, the envisioned fields of applications for both the fundamental and applied studies described in this thesis as well as of the two developed miniaturized devices are discussed.

\section{References}

[1] Neumann, E. et al. (1982). EMBO J. 1 (7), pp. 841-845.

[2] Neumann, E. et al., Electroporation and Electrofusion in Cell Biology. Plenum Press: New York, USA, 1989.

[3] Weaver, J.C. \& Chizmadzhev, Y.A. (1996). Bioelectrochem. Bioenerg. 41 (2), pp. 135-160.

[4] Zimmermann, U. et al. (1976). BBA 436 (2), pp. 460-474.

[5] Baker, P.F. \& Knight, D.E. (1978). J. Physiol.-London 284 (Nov), pp. 30-31.

[6] Gauger, B. \& Bentrup, F.W. (1979). J. Membr. Biol. 48 (3), pp. 249-264.

[7] Zimmermann, U. et al. (1980). Bioelectrochem. Bioenerg. 7 (3), pp. 553-574.

[8] Beebe, S.J. et al. (2002). IEEE T.Plasma Sci. 30 (1), pp. 286-292.

[9] Schoenbach, K.H. et al. (2001). Bioelectromagnetics 22 (6), pp. 440-448.

[10] Schoenbach, K.H. et al. (2004). P.IEEE 92 (7), pp. 1122-1137.

[11] Vernier, P.T. et al. (2004). FEBS Lett. 572 (1-3), pp. 103-108.

[12] Vernier, P.T. et al. (2004). Biophys. J. 86 (6), pp. 4040-4048.

[13] Valero, A. et al. (2008). Lab Chip 8 (1), pp. 62-67.

[14] Valero, A. (2006). Single Cell Electroporation on Chip. University of Twente, Enschede, ISBN: 90-365-2416-4

[15] Bockmann, R.A. et al. (2008). Biophys. J. 95 (4), pp. 1837-1850.

[16] Marrink, S.J. et al. (2009). BBA-Biomembranes 1788 (1), pp. 149-168.

[17] Tieleman, D.P. (2004). BMC Biochem 5 (1).

[18] Chernomordik, L.V. et al. (1987). BBA 902 (3), pp. 360-373.

[19] Hibino, M. et al. (1993). Biophys. J. 64 (6), pp. 1789-1800.

[20] Koronkiewicz, S. et al. (2002). BBA-Biomembranes 1561 (2), pp. 222-229.

[21] Melikov, K.C. et al. (2001). Biophys. J. 80 (4), pp. 1829-1836. 


\section{Chapter 2}

\section{Cell electroporation}

This chapter focuses on the technique of electroporation which is employed to permeabilize a cell membrane. The technique relies on the application of an electric field across the cell to allow for the transport of molecules through the cell membrane. In the first section, the motivation for cell membrane poration is explained. Following this, several possible techniques to pass the cell membrane are presented. This section is followed by an in-depth discussion about the technique of electroporation, along with the most acknowledged model to explain the pore formation process. Last, the limitations of the electroporation technique as well as possible routes towards its improvement are discussed. 


\section{Introduction}

\section{A. Cell membrane}

A cell and its organelles (such as the nucleus, mitochondria or endoplasmic reticulum) are delimited by membranes. These membranes not only define the shape of the structures they enclose, but also make up the boundaries between their content and the environment. The membrane that surrounds the entire cell is called the plasma membrane. It acts as a highly selective barrier that actively controls the transport of species between the intra- and extra-cellular media notably with the help of membrane protein channels. By doing this, the cell maintains a homeostasis with well-defined ion concentrations on both sides of its membrane. This difference in ion concentrations results in a potential difference of $-70 \mathrm{mV}$ across the cell membrane [1].

\section{B. Transport of molecules}

A number of biotechnological and medical applications (such as drug delivery, particle delivery, and gene transfection) rely on the introduction of foreign substances into a cell, and this requires a method to go through the cell membrane. To achieve this, the plasma membrane can be transiently permeabilized [2] or the molecules can be transported into the cell with the help of (i) particle bombardment (DNA coated tungsten- or goldnanoparticles [3-5]), (ii) viruses [2] or (iii) chemicals, such as liposomes, 2-(diethylamino)ether (DEAE)dextrans or calcium phosphate $[2,6]$. The viral and chemical methods possess several major drawbacks. Viral delivery is not only toxic for cells, but is also restricted to specific cell types, it has limited DNA carrying capacity, presents production and packaging problems, can result in recombination of the viral and the cellular genes and, finally, is expensive [2]. The application of chemicals for transfection is precluded by both the cytotoxity of the used reagent and by its difficult application in vivo [2]. Cell poration techniques do not present such limitations; they are less toxic to cells if the pore formation process is properly controlled, and their cost is reduced. In the next paragraph several poration techniques are presented.

\section{(i) Cell poration techniques}

A cell membrane can be porated (i) mechanically, with either a sharp tip or acoustic waves, (ii) optically, using laser beams, (iii) chemically, with detergents or digitonin or (iv) electrically, by means of applied electric field pulses.

\section{Mechanical poration}

Microinjection, the first mechanical technique discussed here, relies on the use of a sharp structure to pierce the membrane and successively load the cells with chemicals. For instance an AFM tip functionalized with a carbon nano-tube (CNT) [7] or a glass micropipette [8-10] have been reported, and molecules to be transferred into the cell are coated onto the CNT, or injected through the pipette, respectively. In the first case, the CNT is 
brought to the cell membrane to pierce it with the help of the AFM set-up which ensures a $\mathrm{nm}$-scale resolution. As CNTs are very small (1-20 nm in diameter) the amount of damage to the cell membrane is reported to be minimal [7]. In the second case, the thin extremity of the pipette is positioned onto the cell surface with the help of micromanipulators and lowered until it is pushed through the cell membrane $[8,10]$. The combination of the microinjection with a special dosing system, an attoliter syringe, enables to precisely control both the dosage down to the aL level of the reagent into the cell and the timing of the process [9]. For both techniques, specific cell targeting is possible as cells are pierced individually. However, suspended cells must be trapped first to facilitate the injection step. The actual immobilization and subsequent piercing of immobilized cells requires a complicated set-up or a highly skilled operator [8]. Consequently, the cell treatment throughput remains low [8, 10]. The poration and injection speed can be increased with automated micro-injectors [11] or by designing micro-fluidic devices where cells are automatically positioned onto a needle [8], but this is still at a developmental stage. Furthermore, the relatively large size of the microneedles (internal diameter in the $\mu \mathrm{m}$ range) as opposed to the CNT's is likely to damage the cell membrane upon piercing [12].

The second mechanical technique, sonoporation, relies on the implosion of (micro)bubbles, which leads to the formation of shockwaves and the emerging micro streaming phenomena result in shear stress on the cells. Thereby, the cell is "massaged" and pores are created in the membrane [13-16]. A key-issue in this technique is the distance between the bubble and the cells. At a too short distance the cells are lysed [17]. When the distance is optimal, pores are large enough to let foreign entities enter the cell but small enough to be transient, allowing the cell to recover. This technique can easily be applied both in vitro and in vivo, since ultrasound can be focused deep into tissues [15-16]. Besides, laser-induced acoustic waves can treat a large number of cells simultaneously [13, 15]. Unfortunately, stresses induced with this technique are not limited to the plasma membrane and can damage internal structures [15]. In vitro sonoporation is mostly applied for adherent cells as suspension cells follow the flow generated by the bubble expansion and collapse and, as a result, experience little shear stress [15]. The recent development of micro-fluidic structures that enable the confinement of suspension cells, restricting their movement, extends the applicability of the technique [13].

\section{Optical poration}

Optoporation makes use of a focused laser beam to directly create holes in a cell membrane [18]. This technique benefits from the fact that a laser beam can be focused with great precision on a certain nm-sized location [15]. However, the precise mechanism for pore creation is still unclear. As is also the case with sonoporation, optoporation is considered to be a mild treatment as it does not involve any contact with the cell [19]. Furthermore, it can be applied to both adherent and suspension cells [18]. Similar to microinjection, cells are treated individually and only certain cells from a multi-cell culture can be targeted. Nevertheless, as for mechanical techniques, the throughput might be 
limited by the time needed to precisely align the laser beam, although automation can again help speeding up the process. A novel approach to optoporation employs the irradiation of multiple cells that are coated with light-absorbing nano-particles attached to their membranes [20]. The illumination of these particles results in local heating of the medium giving rise to pore formation in the cell membrane. Although the throughput is increased this way, the membrane is permeabilized to a much larger extend than with normal optoporation. However, cell specific targeting is more straightforward, since the particles can be attached specifically to certain cells [15].

\section{Electrical poration}

The electrical poration of cell membranes, electroporation (EP), relies on the application of short pulses of a high electric field. Cells are permeabilized with nothing more than two electrodes and a voltage source. However, all cells have a different critical transmembrane potential for the onset of pore formation; as a result, the same electric field parameters can cause irreversible electroporation for certain cells while being applied for reversible electroporation in other cells. Electroporation can not only target the plasma membrane of both adherent and suspension cells, but also the internal membranes by decreasing the length of the pulses to the ns-range [21-23]. This technique is discussed in full detail in the next section of this chapter.

\section{Electroporation}

One goal of this thesis is to better understand the molecular events leading to the membrane electropermeabilization and to identify the parameters that affect this process. In the next paragraph, a more elaborate description of the technique of electroporation as well as its applications is given. This is followed by a theoretical model describing the steps involved in the formation and resealing of the pores and the molecular transport through them. In the last section, the current limitations of the technique are discussed together with possible ways to improve it.

\section{A. Technique}

The application of an electric field onto a cell results in charging of its membrane. The largest membrane potential is found at the poles of the cell facing the electrodes, whose magnitude depends on the strength of the applied field [24] (see Figure 2.1). When the transmembrane potential exceeds the critical value of $0.2-1 \mathrm{~V}$, a rapid rise in the membrane's conductance is measured, indicating the creation of current pathways [25-26]. Three scenarios are possible when an electric field is applied to a cell. (i) When only a weak treatment is adopted, i.e. very short or weak pulses, no detectable change takes place in the membrane (absence of pores or too small pores) (Figure 2.1A). (ii) When the treatment is drastic, i.e. if very high and long fields are applied, the cell is lysed (irreversible electroporation) (Figure 2.1C) [27-28]. (iii) When the electric treatment lies in between and consists of short DC pulses in the ns-ms range or exponentially decaying pulses in the order 
of $\mathrm{kV} / \mathrm{cm}[24]$, the potential across the membrane just exceeds the critical value and the jump in conductance remains transient (reversible electroporation) (Figure 2.1B). In this scenario, a short-lived rearrangement in the molecular structure of the membrane leads to the formation of pores and, consequently, to a substantial increase in the cell permeability to foreign entities [26, 29-31]. Membrane recovery starts after termination of the pulse, allowing the cell to survive and possibly enclose any molecule added in the solution [30, 3234].

Next to the formation of pores in the membrane, the application of an electric field can have additional but related effects on the membrane. Firstly, the electroporation signal promotes the process of endocytosis in the porated areas of the cell membrane up to one hour after the application of the pulse [35]. In vesicles, an additional phenomenon has been observed: the formation of nanotubules upon the application of a low electric field (2 - 200 $\mathrm{V} / \mathrm{cm})[36]$.

Originally, electroporation has been developed to transfect bacteria. To create functional colonies, only one transfected and surviving cell is needed, so a high viability of the transfected cells has not been a major point of interest [37]. Only when the transfection of mammalian cells has become more popular, cell survival has started to be an issue. Still, although the use of electroporation for the transfection of mammalian cells has been increasing the last few decades [38-39], the overall success rate of the process remains low. Using a batch protocol (see below) typically only 40 to $70 \%$ of the cells are viably electroporated [40], the largest part remains viable while being unaffected and a small amount of cells dies. This is partly a result of the equipment used to electroporate the cells. Electroporation is traditionally performed in a cuvette equipped with two electrodes opposite from each other separated by a distance of a few millimeters up to one centimeter. To reach the critical transmembrane potential of $0.2-1 \mathrm{~V}$, a typical high voltage of $10^{2}-10^{3}$ $\mathrm{V}$ is applied across the cuvette. The whole process is difficult to control at the level of a cell population as the outcome of the electroporation experiments notably depends on a number of cell-related parameters, such as the cell size, shape, for instance. Furthermore, all cells feel a different electric field, since not only the distance to the electrodes is different for each cell and the field is also less uniform depending on the cell density but also because it depends on the cell orientation with respect to the electric field. Possible methods to increase the control over the electroporation process are discussed in section IIC. 


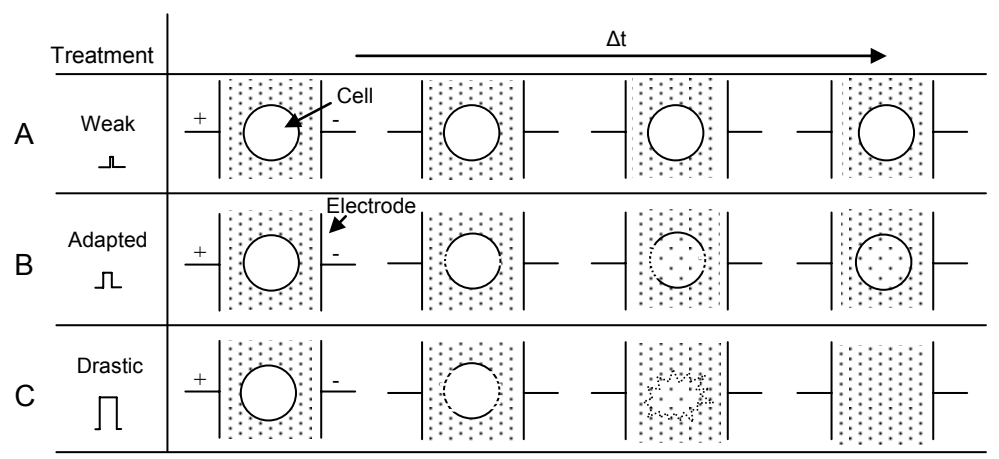

Figure 2.1 Schematic representation of the three possible scenarios of electroporation.

A) Weak treatment: the applied electric field is too low; the transmembrane potential does not reach its critical value. Subsequently, the cell is not permeabilized or pores are too small to be detected. B) Intermediate treatment: the transmembrane potential does reach its critical value and a finite amount of pores is created in the membrane. The cell membrane reseals and electroporation is successful (reversible electroporation). C) Drastic treatment: the applied electric field is too strong. Many and large pores are formed in the membrane. The cell dies (irreversible electroporation).

\section{B. Applications}

Traditionally, electroporation takes place in vitro; a cell suspension is placed in a cuvette equipped with two electrodes on which a high voltage (in the $\mathrm{kV}$ range) is applied. This conventional approach is mainly employed for the transport of molecules across the cell membrane, such as genes, drugs and particles [26, 29] but also as for sterilization purposes with the irreversible electroporation of bacteria [27].

These applications require the cells to be placed in suspension outside an organism; in other words, the cell must be isolated from their natural environment prior to the treatment. This limits the treatment possibilities to cells that can easily be retrieved from their natural environment, electroporated and placed back later. Applications to cells that cannot be removed from an organism, another approach has emerged for the in vivo treatment of cells. The popularity of the technique of electroporation for the treatment of the skin or tissues close to it has been increasing tremendously over the last two decades both for gene transfection as well as cancer treatment purposes (electro(chemo)therapy) [37].

\section{Theory describing the electroporation process}

Although electroporation has been widely employed for many years, precise knowledge about the fundamental mechanisms leading to the permeabilization in the membrane is still lacking. Still, various models have been developed to describe the nucleation and growth of pores in membranes; these models often concern planar models of cell membranes which lend themselves to experimentation and computation-based 
modelling. The coming section introduces the most accepted theory on the pore formation process and the transport of substances through these pores.

\section{(i) Transient aqueous pore model}

The transient aqueous pore model describes electropermeabilization as the growth of initial hydrophobic defects into hydrophilic pores in a planar membrane. As this model includes both the stochastic nature of the permeabilization and the dependency of the pore lifetime on the transmembrane potential it is recognized as the most realistic [31].

The basic principle behind this theory is that lipids are replaced by water molecules in a pore, and this costs a certain amount of energy. In theory, surpassing this barrier could happen due to thermal fluctuations although the probability of this event is very small as the amount of energy provided to the membrane by these fluctuations is too small at room temperature. However, this energy barrier can be lowered by application of a potential across the membrane, and the probability for pore formation increases non-linearly [31, 38].

Molecular dynamics simulations (MDS) on simple phospholipid systems using this theory have demonstrated that the actual formation of a pore proceeds in three steps upon application of a voltage across the membrane (see figure 2.2). In the first stage, the electric field is locally enhanced, causing water defects in the bilayer structure. In the second stage, water molecules form a water file that spans the bilayer by establishing hydrogen bonds with each other, resulting in the formation of a hydrophobic pore. In the last stage, molecular rearrangement of the phospholipids in the vicinity of this water defect occurs and phospholipid molecules move towards this water channel to give a hydrophilic pore lined with phospholipid head groups [41-42]. A pore can either grow unlimitedly until it reaches a boundary (rupture) or close again after the transmembrane potential returns back to below its critical value. Pores are widely spread in planar membranes, making pore-pore encounters unlikely. The formation of a pore results in the local decrease of the transmembrane potential around it, which reduces the likelihood for a second pore to form near the first one. For the same reason, a second pore would grow faster in the direction opposite to the first pore [31, 43]. As more and more pores form, the membrane starts to discharge as a result of the current leakage through the pores. Consequently, the overall transmembrane potential is stabilized, and decreases. No more pores are created, but existing pores still go on growing slowly for some time [44]. At the end of the pulse, the transmembrane voltage decreases back to its initial value, and pores start to shrink. Accordingly, the conductance of the membrane decreases, indicating the beginning of the recovery process [44]. 
1

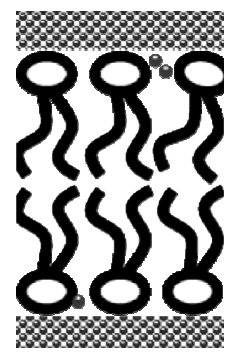

2

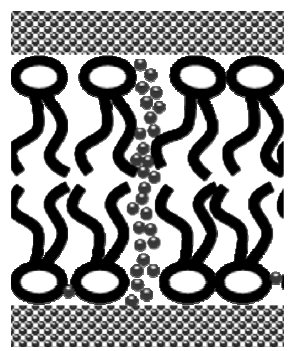

3

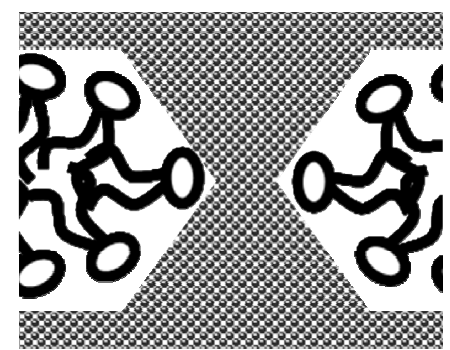

Figure 2.2. Pore formation process.

Three steps involved in pore formation process as demonstrated by MDS studies. 1) The creation of water defects. 2) The formation of a water file through the membrane (hydrophobic pore) 3) The rearrangement of the phospholipids resulting in a hydrophilic pore lined with phospholipid head groups.

The transient aqueous pore model is valid for planar homogenous membranes but for vesicles and cell membranes the situation is more complicated as the electric field is not homogeneous throughout the entire membrane. The transmembrane potential depends on the angle between the membrane and the field and the size of the vesicle or cell, as shown in equation. 2.1:

$$
U=1.5 \cdot r \cdot E_{0} \cdot \cos \theta
$$

with $U$ the transmembrane potential, $r$ the radius of the cell, $E_{0}$ the applied electric field and $\theta$ the angle between the electric field and the membrane (Figures 2.3 and 2.4) [31]. Since the resting potential of cells is $-70 \mathrm{mV}$ on the inside, the critical transmembrane potential is reached first at the side of the anode, and consequently the first pores appear in the pole of the membrane facing that electrode [45]. The pores are formed in and migrate to areas of large transmembrane potential, which correspond to the poles facing the electrodes (see Figure 2.3). As a consequence, in the case of vesicles and cells, pores merge to form more complex structures [43-44]. A recent model proposed by Krassowska and Filev showed that, although the highest pore density is found at the poles, the size of those pores is much smaller than those located at the border of the electroporated region (the areas the furthest away from the electrodes where the transmembrane potential just exceeds its critical value).

Nevertheless, pores in the area away from the electrodes go on growing to compensate for their small number and finally form the largest part of the total pore area. As a consequence, the conductivity in that region of the membrane is higher than at the poles. Another important difference between planar and spherical membranes is the existence of a boundary or not to take up the excess of material and the possibility to completely break. In BLMs a boundary is formed by the annulus, and/or the aperture, but in spherical objects no boundary is present. In other words, spontaneous rupture of the entire membrane is not possible in a vesicle [31,43]. For cells, the situation is again more complex. The cell membrane can only partially be ruptured, as any structure interacting with it, such as the 
cytoskeleton, might locally define the boundary required for rupture [31, 43, 46]. However, the main mechanism that causes irreversible electroporation is the rupture of the membrane due to osmotic pressure and extensive swelling of the cell.

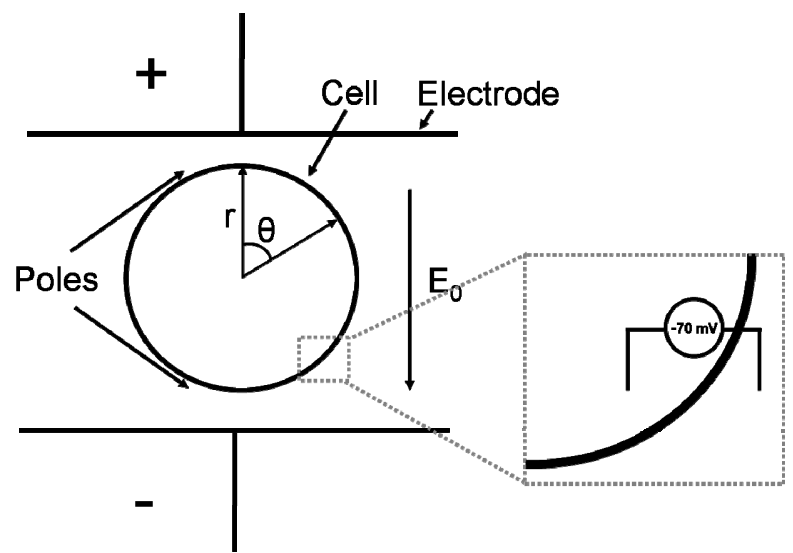

Figure 2.3 Transmembrane potential.

Schematic representation of the dependence of the transmembrane potential on the size of the cell, the position on the membrane and the applied the electric field with $\mathrm{r}$ the radius of the cell, $\mathrm{E}_{0}$ the applied electric field and $\theta$ the angle between the electric field and the membrane. Since the resting potential of cells is $-70 \mathrm{mV}$, the critical transmembrane potential is reached first at the side of the anode, where pore formation starts.

The lifetime and the size of the pores in both planar and spherical membranes have been determined using both computation and experimental results. Pores start to form nano- to microseconds after application of the electric field [42, 45, 47-48] and expand in a few milliseconds. The diameter of an electropore varies from 0.5 to $400 \mathrm{~nm}$ [42, 44-45, 47]. Pores shrink rapidly but close slowly until the membrane returns to its original state within seconds to minutes in cells and milliseconds to seconds in artificial membranes [48-53]. The time required for pores to reseal depends on the amount of applied pulses and their duration, but not on the strength of the electric field [54]. Besides, in bilayer lipid membranes, the recovery process is highly temperature dependent $[44,53]$, whereas, in cell membranes, active processes may also be involved [44] as well as the effect of the cytoskeleton on the pore closure process.

To summarize, the transient aqueous pore theory of electroporation has already provided insight in many different aspects of the process of pore formation, such as the dependence of the probability for pore formation on the transmembrane potential, the timescale involved and the size of the resulting pores. Using MDS, molecular events leading to the formation of the pores have been unraveled. Nevertheless, knowledge about the precise effects of the many constituents of a cell membrane and interactions between them is still missing. 


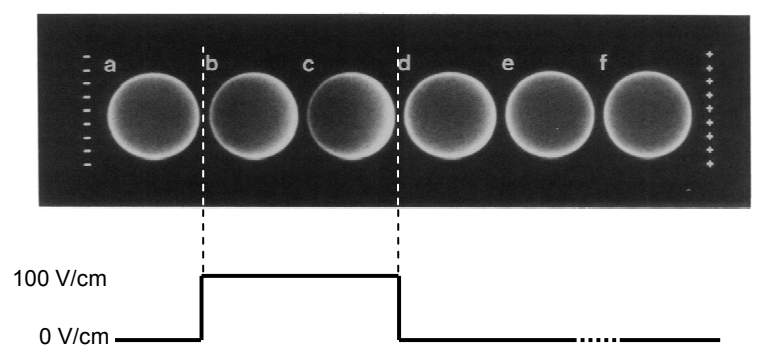

Figure 2.4. Membrane potential.

Fluorescence images of a sea urchin egg stained with a voltage sensitive dye (RH292), showing the distribution of the transmembrane potential in the membrane: a) before the application of an electric pulse, $b-c) 1$ and $5 \mu \mathrm{s}$ after the application of a pulse, $d-f) 1 \mu \mathrm{s}, 5 \mu \mathrm{s}$, and $2 \mathrm{~s}$ after the end of the pulse. Adapted from [48].

\section{(ii) Molecular transport}

The transport across the membrane combines two different processes: diffusion, electrophoresis $[31,38,44]$. The last mechanism is only found when a difference in potential is present; this can be either the resting potential of the cell $[38,44]$ or an externally applied voltage [55]. Transport through the membrane can occur in both directions, and this can be different for both poles of a cell [44]. The uptake of small molecules is purely based on diffusion while large molecules, such as DNA and proteins however, require more complex transportation mechanisms. Firstly, DNA must be unrolled before it fits through the pores. Furthermore, these molecules interact with the membranes and possibly reside transiently inside the bilayer structure (protruding on both sides) before being electrophoretically pulled through [56]. The presence of such long molecules inside the membrane also enhances the uptake of small molecules by inhibiting the resealing of the pore [44]. For the transport of large molecules, such as DNA, weak pulses are the most appropriate as higher pulses only favor the formation of a large amount of small pores at the poles of the cell and not of large pores at the edge of the electroporated region (see Figure 2.5) [45]. Furthermore, the application of weak but long pulses promotes the transport of molecules across the membrane not only by diffusion, but also by electrophoresis.

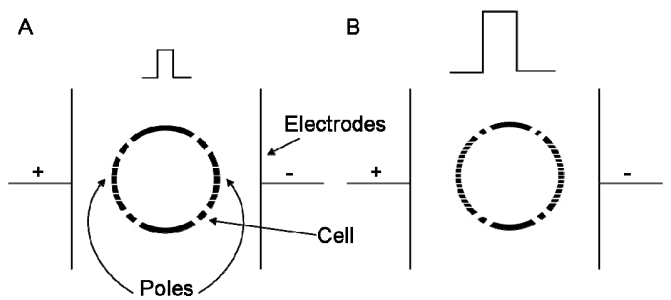

Figure 2.5. Pore size distribution as a function of the applied field.

A) Upon application of a weak electric field, small pores are created at the poles of the cell membrane facing the electrodes, and large pores at the edge of the porated regions. B) When the pulse strength is increased, more small pores are created, whereas the amount of large pores is unchanged. 


\section{Improving the process}

Electroporation is a relatively simple, straightforward and efficient method for membrane permeabilization and for the subsequent introduction of entities into a cell [29]. This technique lends itself to the treatment not only of large populations of cells, but also of individual cells [44]. Besides, electroporation alleviates the use of chemicals, viruses and complicated protocols. Nevertheless, other methods, like chemical or viral transfections, remain more popular for cellular engineering. A possible reason for this is that the technique of electroporation still presents a number of limitations that must be overcome before it becomes a routine technique.

Firstly, the relative success yield for viable transfection is low. Increasing this success rate may be facilitated by a deeper knowledge about the effect of the application of an electric field on the plasma membrane. This is however not straightforward, not only because of the complexity of the physical phenomena taking place in the membrane [31, 4445], but also due to a lack of techniques available to visualize them [31]. To get a better insight into the pore formation process and the transport of molecules, two different directions are possible, either via theoretical studies (MDS) or by experimental means. In both cases, simplified models are required to facilitate the study of the pore formation process. These models have a simpler structure, and the applied electric field is homogenous throughout the whole membrane. For MDS studies, models consist of small membrane patches, whereas for experimental investigations, planar models of cell membranes, i.e. bilayer lipid membranes (BLMs) are employed. These membrane models and their applications are discussed in detail in chapter 3 whereas experimental studies performed on both model membranes and cells are discussed in chapters 4-6 and chapter 8 of this thesis, respectively.

Furthermore, in conventional set-ups, the electric field required to reach the critical potential value across the membrane is provided by a high voltage source. The latter brings along unwanted danger and requires special precautions. Fortunately, these high voltage sources can be alleviated. By miniaturizing the device (e.g., by using micrometer-sized electrodes or microfluidic devices), the gap between the electrodes becomes smaller and as a consequence a lower total potential is necessary to reach the critical value of the transmembrane potential. For instance, the distance between the electrodes is no more than several hundreds of micrometers and the applied potential in the order of a few volts. A direct benefit of miniaturization is the possibility to study the response of individual (or a few) cell(s) and derive information on the heterogeneity of cell populations. Conversely, when individually addressable electrodes are used for single cell treatment, the electroporation protocol can be adjusted at the single cell level, increasing the overall yield of the process. An overview of miniaturized devices is given in Appendix A.

\section{Conclusion}

A cell membrane is impermeable to foreign molecules, such as drugs, genes, particles 
and certain dyes. Their transport into cells requires mild permeabilization of the cell membrane. A technique to achieve this is electroporation, which relies on the application of electrical pulses. When the transmembrane potential reaches the threshold value of $0.2-1 \mathrm{~V}$, small perturbations (hydrophobic pores) evolve into transient aqueous pathways (hydrophilic pores) where transport can occur across the membrane. The precise molecular processes in the membrane leading to the formation of pores (and the subsequent transport of molecules) are not yet fully understood. Nevertheless, models have been proposed to describe them. For pore formation, the most accepted model is the transient aqueous pore model. In this model, the probability for pore formation is described as a function of the transmembrane potential, along with the effect of the electrical signal on the nucleation and growth of the pores. Despite these attempts to better understand phenomena occurring in the membrane, the overall success yield for viable transfection is still low. Since each cell responds differently to the applied field, the outcome of the electroporation process is difficult to predict and to control, even at the single cell level. Two possible approaches are proposed to remedy this. Firstly, by studying electroporation with planar models of cell membranes, the processes involved in the pore formation process can be better envisioned, such as for instance the influence of both the individual membrane constituents and interactions between them. Secondly, the use of miniaturized devices to electroporate (single) cells allows for shaping the electric field and generates a better controlled environment, thereby tuning it for optimal transfection and viability at the single cell level.

\section{References}

[1] Alberts, B. et al. (1989). Molecular Biology of the Cell. Garland Publishing, Inc: New York, NY, USA

[2] Luo, D. \& Saltzman, W.M. (2000). Nat. Biotechnol. 18 (1), pp. 33-37.

[3] Klein, T.M. et al. (1987). Nature 327 (6117), pp. 70-73.

[4] Uchida, M. et al. (2009). Biochimica Et Biophysica Acta-General Subjects 1790 (8), pp. 754-764.

[5] Yang, N.S. (1992). Crit. Rev. Biotechnol. 12 (4), pp. 335-356.

[6] Graham, F.L. \& Vandereb, A.J. (1973). Virology 52 (2), pp. 456-467.

[7] Chen, X. et al. (2007). Proc. Natl. Acad. Sci. USA 104 (20), pp. 8218-8222.

[8] Adamo, A. \& Jensen, K.F. (2008). Lab Chip 8 (8), pp. 1258-1261.

[9] Laforge, F.O. et al. (2007). Proc. Natl. Acad. Sci. USA 104 (29), pp. 11895-11900.

[10] Zhang, Y. \& Yu, L.C. (2008). Bioessays 30 (6), pp. 606-610.

[11] Wang, J. et al. (2007). Anal. Chem. 79 (24), pp. 9584-9587.

[12] Sakaki, K. et al. (2009). IEEE Trans. Biomed. Eng. 56 (8), pp. 2064-2074.

[13] Le Gac, S. et al. (2007). Lab Chip 7 (12), pp. 1666-1672.

[14] Miller, M.W. et al. (1996). Ultrasound Med. Biol. 22 (9), pp. 1131-1154.

[15] Yao, C.P. et al. (2008). IEEE Trans. NanoBiosci. 7 (2), pp. 111-119.

[16] Wu, J. \& Nyborg, W.L. (2008). Adv. Drug Del. Rev. 60 (10), pp. 1103-1116.

[17] Dijkink, R. et al. (2008). Phys. Med. Biol. 53 (2), pp. 375-390.

[18] Stevenson, D. et al. (2006). Opt. Express 14 (16), pp. 7125-7133. 
[19] Palumbo, G. et al. (1996). J. Photochem. Photobiol. B 36 (1), pp. 41-46.

[20] Hüttmann, G. et al. (2005). Med. Laser Appl. 20 (2), pp. 135-139.

[21] Kolb, J.F. et al. (2006). Bioelectromagnetics 27 (3), pp. 172-187.

[22] Vasilkoski, Z. et al. (2006). Phys. Rev. E 74 (2).

[23] White, J.A. et al. (2004). J. Biol. Chem. 279 (22), pp. 22964-22972.

[24] Chang, D.C. et al., Guide to Electroporation and Electrofusion. Academic Press, Inc.: San Diego, CA, USA, 1992.

[25] Zimmermann, U. et al. (1974). Biophys. J. 14 (11), pp. 881-899.

[26] Zimmermann, U. et al. (1976). BBA 436 (2), pp. 460-474.

[27] Sale, A.J.H. \& Hamilton, W.A. (1967). BBA 148 (3), pp. 781-788.

[28] Sale, A.J.H. \& Hamilton, W.A. (1968). BBA 163 (1), pp. 37-43.

[29] Neumann, E. et al. (1982). EMBO J. 1 (7), pp. 841-845.

[30] Neumann, E. et al., Electroporation and Electrofusion in Cell Biology. Plenum Press: New York, USA, 1989.

[31] Weaver, J.C. \& Chizmadzhev, Y.A. (1996). Bioelectrochem. Bioenerg. 41 (2), pp. 135-160.

[32] Baker, P.F. \& Knight, D.E. (1978). J. Physiol.-London 284 (Nov), pp. P30-P31.

[33] Gauger, B. \& Bentrup, F.W. (1979). J. Membr. Biol. 48 (3), pp. 249-264.

[34] Zimmermann, U. et al. (1980). Bioelectrochem. Bioenerg. 7 (3), pp. 553-574.

[35] Rols, M.P. et al. (1995). Biochem. Biophys. Res. Commun. 208 (1), pp. 26-35.

[36] Castillo, J.A. et al. (2009). Langmuir 25 (1), pp. 391-396.

[37] Gehl, J. (2003). Acta Physiol. Scand. 177 (4), pp. 437-447.

[38] Chen, C. et al. (2006). Med Biol Eng Comput 44 (1-2), pp. 5-14.

[39] Golzio, M. et al. (2001). Bioelectrochemistry 53 (1), pp. 25-34.

[40] Chen, C. et al. (2008). Phys. Med. Biol. 53 (17), pp. 4747-4757.

[41] Marrink, S.J. et al. (2009). BBA-Biomembranes 1788 (1), pp. 149-168.

[42] Tieleman, D.P. (2004). BMC Biochem 5.

[43] Freeman, S.A. et al. (1994). Biophys. J. 67 (1), pp. 42-56.

[44] Weaver, J.C. (2003). IEEE T. Dielect. El. In. 10 (5), pp. 754-768.

[45] Krassowska, W. \& Filev, P.D. (2007). Biophys. J. 92 (2), pp. 404-417.

[46] Isambert, H. (1998). Phys. Rev. Lett. 80 (15), pp. 3404-3407.

[47] Bockmann, R.A. et al. (2008). Biophys. J. 95 (4), pp. 1837-1850.

[48] Hibino, M. et al. (1993). Biophys. J. 64 (6), pp. 1789-1800.

[49] Barnett, A. \& Weaver, J.C. (1991). Bioelectrochem. Bioenerg. 25 (2), pp. 163-182.

[50] Chernomordik, L.V. et al. (1987). BBA 902 (3), pp. 360-373.

[51] Koronkiewicz, S. et al. (2002). BBA-Biomembranes 1561 (2), pp. 222-229.

[52] Melikov, K.C. et al. (2001). Biophys. J. 80 (4), pp. 1829-1836.

[53] Saulis, G. et al. (1991). Bioelectrochem. Bioenerg. 26 (1), pp. 1-13.

[54] Rols, M.P. \& Teissie, J. (1990). Biophys. J. 58 (5), pp. 1089-1098.

[55] Ionescu-Zanetti, C. et al. (2008). Biomed. Microdevices 10 (1), pp. 113-116.

[56] Rols, M.P. (2006). BBA-Biomembranes 1758 (3), pp. 423-428. 


\section{Chapter 3}

\section{Bilayer lipid membranes}

\section{From a conventional set-up towards "high-throughput" integrated microfluidic platforms}

This chapter focuses on bilayer lipid membranes (BLMs). BLMs are simplified planar models of cell membranes, commonly employed for both fundamental and applied studies. BLMs are of great importance for improving our understanding of membrane properties or for drug screening purposes, after insertion of a membrane protein of interest. This chapter starts with a motivation for the use of such simplified models, and following this, their conventional preparation techniques and possible applications are presented. The second section of the chapter concerns the recent trend in miniaturizing devices for BLM experimentation and their implementation in a microfluidic platform. Firstly, the advantages brought by the miniaturization and the use of a microfluidic environment are listed. Thereafter, an overview is provided of existing miniaturized and microfluidic devices for BLM experimentation and their potential applications. Lastly, the promising future of miniaturized and microfluidic BLM systems is discussed. 


\section{Introduction}

Bilayer lipid membranes (BLMs) are simplified models of cell membranes since the latter are very complex structures. A BLM is a two-dimensional planar sheet formed by the spontaneous self-assembly of phospholipid molecules into a bilayer, either across a small aperture in a substrate or on the substrate itself [1-2]. BLMs can be adopted for different applications: (i) for fundamental studies on membrane properties, such as their thickness and the packing density of the phospholipids or (ii) as templates for (single) protein studies e.g., drug screening assays, sensing applications and as natural nanopores.

Conventionally, BLMs are prepared (across an aperture) in a vertical substrate, clamped between two buffer-containing chambers (suspended membranes). Several techniques are available to make such membranes, and each method presents its advantages and drawbacks, as discussed in section IIC of this chapter. As optical imaging of vertical substrates is not straightforward and requires dedicated set-ups, BLM characterization is mostly limited to electrical measurements. Nevertheless, optical studies bring additional information, for instance to precisely determine the membrane surface area, the mobility of the phospholipids (using FRAP imaging (Fluorescence recovery after photobleaching)) or the clustering of proteins. For optical measurements, the substrate containing the BLM must be mounted horizontally on the stage of a microscope. This requirement has led to the development of devices containing horizontal substrates and a transparent bottom.

Two decades ago, a novel trend emerged, towards the miniaturization of the BLM experimentation [3]. This miniaturization trend started with downscaling the apertures across which the membranes are prepared. Currently, whole set-ups are becoming miniaturized, containing $\mu \mathrm{L}$-scale reservoirs that lead to the development of completely microfluidic platforms. This novel miniaturization trend has been driven by the emergence of new applications requiring multiplexed and automated measurement platforms, such as the high throughput screening (HTS) of drugs for the pharmaceutical industry.

Next to the suspended membranes discussed above, membranes can also be created on a smooth substrate to yield so-called supported membranes. The latter membranes are more stable than their suspended counterparts. However, it is no longer possible to access both sides of the membrane and molecules protruding on both sides of the membranes, such as membrane proteins, cannot insert. Supported membranes are mostly employed as supports for biosensors or for scanning-based imaging techniques e.g., atomic force microscopy (AFM). Supported membranes are not the focus of this chapter, but they are still mentioned if necessary (i.e. to discuss certain membrane preparation techniques or specific applications). For more detailed information about supported membranes, the reader is referred to the review of Castellana and Cremer [4].

In the next section of this chapter (section II), the concept of bilayer lipid membranes, their conventional preparation techniques and applications are discussed. Section III focuses on the miniaturization of BLM experimentation; firstly, the advantages brought by 
miniaturized, microfluidic and integrated systems are considered and an overview of existing miniaturized and/or microfluidic devices is given together with their methods to characterize the BLM formation. The last section (IV) the envisioned future developments and applications for the miniaturized and microfluidic BLM are described.

\section{Bilayer lipid membranes and their experimentation}

\section{A. The structure of cell membranes}

A cell membrane consists of a (phospho)lipid matrix that firstly defines the structure and shape of the cell, but also serves as a substrate for membrane proteins (see figure 3.1). The latter are involved in many biochemical processes, such as the transport of materials and the cell's communication with its environment.

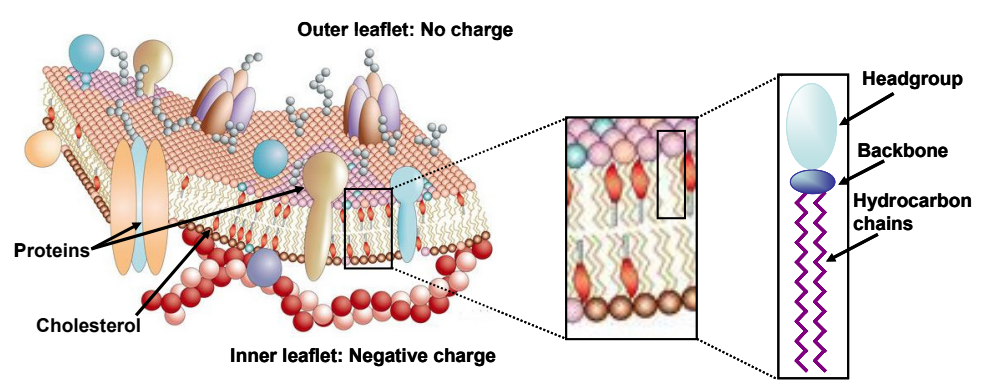

Figure 3.1. The plasma membrane.

A schematic representation of the plasma membrane on the left and a phospholipid molecule on the right. A plasma membrane (Left) consists of a bilayer composed from phospholipid molecules and cholesterol and that functions as a substrate for membrane proteins. A phospholipid molecule is composed of a head group structure that is connected via its backbone to two hydrocarbon tails (Right).

Already in 1925, Gorter and Grendel showed that ghost red blood cells provided enough lipids to form a two molecule-thick layer around the whole cell [5]. They were the first to suggest that the plasma membrane is formed from a bimolecular phospholipid sheet; this idea resulted later in the concept of the lipid bilayer as the basic structure of cell membranes.

Phospholipids (see figure 3.1) are amphiphilic molecules composed of two main parts: (i) a hydrophilic head consisting of a backbone molecule (either glycerol or sphingosine), a phosphate and a polar group and (ii) two "parallel" aliphatic chains (saturated or unsaturated fatty chains, and of various lengths). When phospholipids are immersed in an aqueous solution, they tend to self-assemble into well-defined structures to avoid any contact between the hydrophobic tails and the water. Two configurations are possible in that respect; either the molecules all go to the surface of the solution (with their tails sticking into the air) or they self-assemble into given shapes. In these structures, the tails point towards each other, forming either a micelle if the concentration of the phospholipids is above its critical value or a bilayer (see figure 3.2) [6]. 

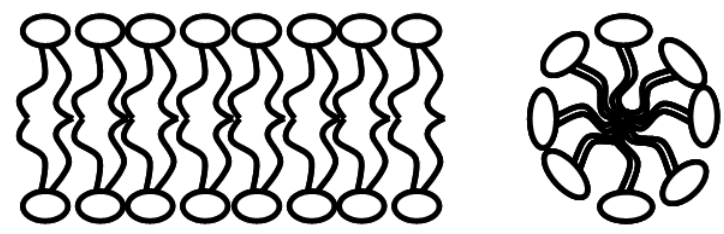

Figure 3.2. Phospholipid self-assembly.

Two possible configurations of phospholipid self-assembly in aqueous solutions. Left: bilayer structure. Right: micellar structure (observed only above the critical micelle concentration (CMC) of the phospholipids).

Inside the bilayer, London-van der Waals forces hold the tails of the phospholipids together. The phospholipids are in a liquid-crystalline state under physiological conditions so that the membrane is fluid and has the consistency of olive oil [6]. This bilayer structure is the only feature all biological membranes have in common, but the actual composition strongly varies between membranes. Hundreds or thousands of different types of phospholipids are described [7] with variations in the length of the hydrocarbon chains, the amount of unsaturations present in the chains, the molecular shape, the nature of the backbone structure and the nature of the head group. These characteristics determine the individual properties of the phospholipids. Phospholipids are firstly classified into two main families, glycerolipids and sphingolipids, according to the nature of their backbone, glycerol and sphingosine, respectively. A second level of classification is linked to the presence of a carbohydrate onto the head groups; those phospholipids are known as glycolipids.

The distinct characteristics of phospholipids affect their packing density and, consequently, the fluidity and stability of the membranes. Besides, the overall structure of the membrane is asymmetrical; not only do the proteins have a specific orientation in the membrane, but the phospholipid distribution of both membrane leaflets does also differ (see figure 3.1). The outer leaflet contains only zwitterionic phospholipids whereas negatively charged phospholipids are found in the inner leaflet.

Another important lipidic constituent of the cell membrane is cholesterol that typically represents $30 \% \mathrm{~mol}$ of the lipid matrix. Cholesterol greatly affects the fluidity of the membrane. Its polar hydroxyl group is positioned close to the phospholipid phosphate head group and its rigid rings interact with the region of the tails located close to the head group [6, 8-9]. Although there is debate on whether cholesterol changes the confirmation of the head groups, all models agree that due to the interaction of the rigid rings with the tails, their mobility is reduced. This increases both the rigidity of the membrane and the phospholipid packing density.

The last main component found in cell membranes is proteins and they account for $50 \%$ weight of the membrane [10]. Membrane proteins contain a hydrophobic part that resides in the hydrophobic core of the membrane. The so-called transmembrane proteins span the 
membrane and present hydrophilic parts protruding on both sides. Cell membranes count two main types of proteins: pore proteins or receptors. The pore proteins transport material through their pore structure across the membrane. Only water, non-polar molecules and some small polar molecules can cross the membrane without assistance; however, channel proteins still actively pump them across the membrane to maintain the cell homeostasis. Conversely, the receptor proteins are responsible for the cell communication with its environment; upon activation by external chemical signals, they trigger signaling cascades in the cell.

\section{B. Bilayer lipid membranes}

As explained in the previous section, a cell membrane is a highly complex structure. Therefore, model systems are employed for a wide variety of studies, in which cholesterol and proteins can be incorporated. Such model membranes can have two possible shapes; planar (bilayer lipid membrane, BLM) or spherical (vesicle) (phospho)lipid bilayers. The development of these model membranes dates back to more than forty years ago, when Rudin and his co-workers made a "black lipid bubble" in an aqueous solution[1]. Their discovery was directly related with the experiments of Hooke and Newton three centuries ago on the appearance and thickness of "black" areas in thinning soap bubbles [6] and the bilayer model of Gorter and Grendel (beginning of the 20th century) [5]. All this led to the realization that the black area of the soap bubble (which is its final stage of thinning before bursting) also consists of a bilayer of amphiphilic molecules.

Planar, suspended BLMs are prepared across a small aperture in a hydrophobic substrate placed between two fluidic compartments. They form a G $\Omega$ seal with the hydrophobic substrate, and this limits any problems of electrical noise. Advantages of such models are numerous. Firstly, both sides of the membrane are easily and independently accessible to both electrical and chemical signals, which is not the case for a cell membrane or a vesicle. Secondly, modulating the cell membrane composition is straightforward. Finally, the membrane can be flat depending on the phospholipid used, which facilitates electrical measurements and modeling. Nowadays, BLMs are not only used for the study on the membrane properties [11-14], but also as platforms for studies on proteins, and notably on (single) channel proteins for sensing [15-16] and drug screening purposes [17].

\section{Techniques for membrane preparation}

Many different techniques are available to prepare bilayer lipid membranes. Suspended membranes, the focus of this chapter, are formed across a small aperture in a hydrophobic substrate, for instance Teflon or poly(methyl methacrylate) (PMMA), or are suspended in a liquid environment. The substrate is conventionally mounted vertically, but recently horizontal substrates have become more and more popular. An advantage of horizontal membranes is their compatibility with optical measurements in addition to conventional electrical measurements, without the need for special optical equipment.

In the next paragraph, the different membrane preparation techniques for both vertical 
and horizontal membranes are introduced, followed by a discussion on the advantages and limitations of these preparation techniques.

\section{(i) Mueller-Rudin technique}

In the Mueller-Rudin technique, the membranes are prepared across an aperture in a (horizontal or vertical) substrate that separates two liquid compartments. Prior to membrane formation, the phospholipids are dissolved in a non-volatile solvent, usually n-decane. The aperture and the substrate are pre-treated with either a pure organic solvent or the phospholipid solution, and the solvent is allowed to evaporate before the substrate is immersed in the buffer solution. Membranes are formed by brushing across the aperture either a small pipette-tip, a thin brush or a thin Teflon stick or sheet, which has been dipped in the phospholipid solution beforehand (see figure 3.3) [1, 18]. The painted solution spreads thinly in the middle of the aperture, resulting in the self-assembly of the phospholipids into a bilayer. As the solvent cannot evaporate, a small and uncontrollable amount of solvent remains between the two phospholipid monolayers and at the edge of the aperture forming a "reservoir" of solvent and lipids, known as the annulus. The presence of a layer of solvent between the two membrane leaflets can be prevented by using specific solvents such as squalene $\left(\mathrm{C}_{30} \mathrm{H}_{50}\right)$ [19]. In that case, the solvent molecules are too large to insert in the bilayer and they move out of the membrane area into the annulus during membrane formation. Unfortunately, squalene does not dissolve all phospholipids, and n-decane is still mostly employed as a solvent for the Mueller-Rudin technique, which limits its applicability [20].

\section{(ii) Langmuir-Blodgett derived techniques}

This class of membrane preparation methods relies on the transfer of phospholipids from a lipid film formed on the surface of a buffer solution onto a substrate. As phospholipids are amphiphilic molecules, they orient themselves in the floating film, their head groups being immersed in the buffer while their hydrocarbon tails stick up in the air. A main advantage of this method is the absence of solvent between the two monolayers, since the solvent evaporates before membrane preparation, although an annulus can still be present [19]. Different techniques employ this principle which are all discussed due to their similarities: (i) the combination of the Langmuir-Blodgett and Langmuir-Schaefer techniques (supported membranes); (ii) the combination of the Langmuir-Blodgett method and vesicle collapsing (supported membranes); (iii) the Montal-Mueller technique (suspended membranes); and (iv) the tip-dip technique (both supported and suspended membranes) as detailed in the following paragraphs. 


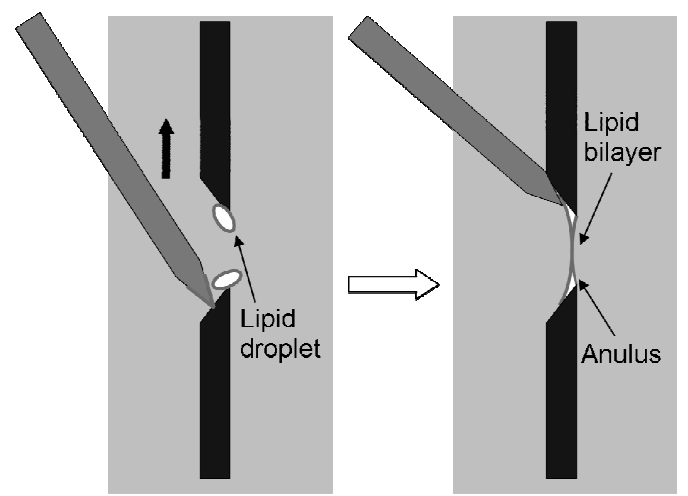

Figure 3.3. Muller-Rudin technique.

The painting technique used to form a suspended membrane. Left: A droplet of lipids dissolved in a solvent is spread across an aperture using a Teflon tip. Right: Due to the thinning of the layer across the aperture, the phospholipids self-assemble into a bilayer.

With the Langmuir-Blodgett and Langmuir-Schaefer technique, a flat vertical substrate is immersed in a buffer solution, after a monolayer of phospholipids has been created on the surface of the aqueous solution. This phospholipid film is achieved by carefully pipetting a small amount of the phospholipid solution onto the surface of the buffer solution and by letting it spread out while the solvent evaporates [21]. Consequently, a more volatile solvent than for the Mueller-Rudin technique is usually employed, such as hexane. When the substrate is pulled out of the buffer solution, a monolayer of phospholipids is formed on each side of the substrate; this is the Langmuir-Blodgett technique. The resulting "coated" substrate is rotated by ninety degrees and pushed on the monolayer of lipids remaining on the buffer surface (Langmuir-Schaefer procedure) and a bilayer is formed as the two phospholipid monolayers bind to each other (see figure 3.4) [2, 4]. An alternative to this technique consists of collapsing a vesicle on the first monolayer to form a second monolayer (see figure 3.5) [22].

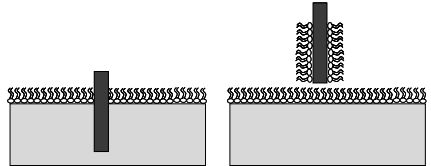

Figure 3.4. Langmuir-Blodgett and LangmuirSchaefer technique.

Left and Middle: Formation of a monolayer of phospholipids on a flat substrate by the LangmuirBlodgett technique. Right: Formation of a bilayer by the Langmuir-Schaefer procedure. The substrate is pushed on the lipid monolayer on the buffer solution
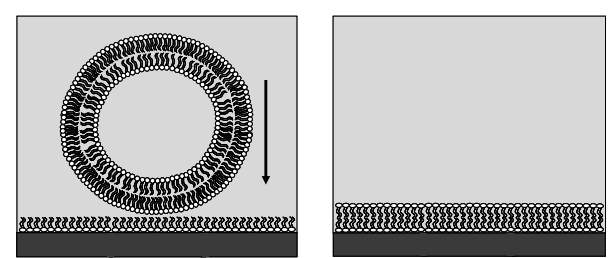

Figure 3.5. Langmuir-Blodgett and vesicle collapse technique.

Left: After a monolayer is formed on a flat substrate using the Langmuir-Blodgett technique (see figure 3.4), vesicles are brought into contact with that monolayer. Right: After vesicle bursting, a bilayer is formed on the substrate. 
The Montal-Mueller technique is employed to make suspended membranes. It requires a pretreatment of the substrate containing the aperture, similarly to the Mueller-Rudin technique; however, this preliminary step is performed here using only organic solvents (e.g., a mixture of hexadecane and hexane) and not lipids [23]. As for the Langmuir-Blodgett technique, a monolayer of phospholipids is formed on the surface of the buffer solution on both sides of the substrate prior to membrane formation. Once the two phospholipid films are formed, the liquid levels on both sides of the substrate are successively and slowly raised and lowered several times, as illustrated in figure 3.6. Inside the aperture, the tails of the phospholipid molecules contact each other and "zip" together to form a bilayer structure (see figure 3.6). This technique presents two main advantages over the Mueller-Rudin technique. Firstly, solvent-less membranes are created, as no solvent is present between the two phospholipid layers. Secondly, as the two liquid compartments are separated by the aperture containing substrate, the phospholipid composition of the two lipid films can be different, resulting in the formation of asymmetrical membranes [24].

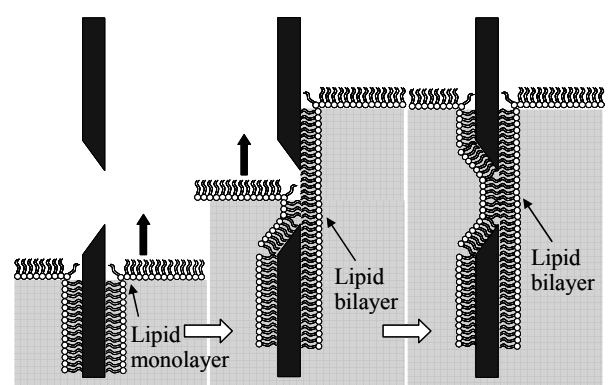

Figure 3.6. Montal-Mueller technique.

Left: A monolayer of phospholipids is formed of top of the buffer on both sides of the substrate. Middle and Right: the liquid levels on both sides of the substrate are raised slowly. Inside the aperture, the tails of the phospholipid molecules contact each other and "zip" together to forming a bilayer structure.

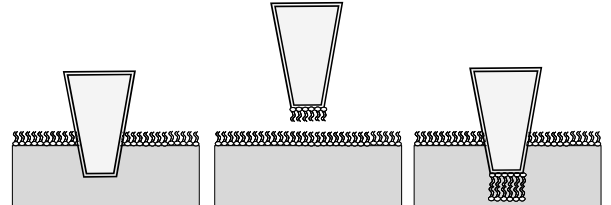

Figure 3.7. Tip-dip technique.

Left and Middle: A monolayer of lipids is formed at the liquid-air interface of the pipette tip by retracting a glass patch clamp pipette from the buffer solution covered with a phospholipid film. Right: When the pipette is dipped again in the buffer reservoir, a bilayer structure is formed.

Alternatively, horizontal suspended membranes can be prepared across the aperture of a capillary tube using the so-called tip-dip technique (see figure 3.7). As for the MontalMueller technique, a film of phospholipids is prepared by evaporation of an organic solvent at the interface of a buffer reservoir around glass patch-clamp that is dipped into the reservoir. The tip is retracted from the buffer solution to leave a monolayer of lipids at the liquid-air interface at the pipette tip. When the pipette is inserted again in the buffer reservoir through the phospholipid monolayer, a second monolayer of phospholipids attaches to the previous one to give a bilayer structure at the pipette tip. As for the MontalMueller technique, the two leaflets of the membranes are created independently, so this technique lends itself well to the formation of asymmetrical membranes [18, 25]. When the 
pipette tip is not filled with a buffer solution, but with an agarose gel prior to the membrane formation, a supported membrane is created. The advantage of such a membrane is that they have a longer lifetime than suspended ones [18].

\section{(iii) Thinning of a lipid plug}

There are two methods reported in the literature that describe the formation membranes by the thinning of a lipid plug [26-28]. These techniques are, on the contrary to several of the previous described techniques, exclusively reported for horizontal substrates.

The air-exposure technique is, on the contrary to several of the previous described techniques, exclusively reported for horizontal substrates. There, the substrate containing the aperture(s) is placed between an upper well and a lower liquid reservoir. Figure 3.8 illustrates how membranes are prepared [26] [27-28]. In a first step, the lower reservoir is filled with buffer solution, while the upper well is kept empty. A small amount of phospholipid solution in n-decane is introduced into the upper well, followed by fresh buffer, resulting in a lipid plug in the aperture. A few seconds after addition of the fresh buffer, the upper well is emptied and the remaining phospholipid layer exposed to air. As a result, the solvent drains away from the aperture and the lipid plug starts to thin and rearrange; since the lipid monolayers at the two interfaces of the plug are attracted to each other by van der Waals forces. As soon as they come into close proximity of each other, they "zip" together, forming a bilayer structure. The thinning of the phospholipid film is monitored optically. When the bilayer occupies $70-80 \%$ of the aperture, fresh buffer is injected in the upper well to stop the thinning process to prevent membrane rupture. The area at the edge of the bilayer structure forms the annulus that stabilizes the membrane. As for the Montal-Mueller technique, a thin layer of buffer probably remains present between the two leaflets and the annulus. Furthermore, as the membrane formation is mostly monitored optically, this technique does not lend itself easily to automation.

In the second technique, a similar set-up is employed, but now the lipid plug is not thinned by the exposure to air but by carefully controlling the application of a hydrostatic pressure [27-28]. Since this technique does not depend on optical monitoring, it lends itself well for automation.

\section{(iv) Vesicle fusion}

The most employed technique to prepare horizontal (mostly supported) BLMs makes use of vesicles, which are burst on a substrate (see figure 3.9). The substrate is hydrophilic to promote the interactions of the phospholipid head groups with it. Several types of vesicles are possibly employed. Vesicles that consist of only phospholipids, such as giant unilammelar vesicles (GUVs), are relatively easy to make. Nevertheless, for many applications, proteins need to be inserted into the membranes, and this is not always straightforward, as is discussed in a later paragraph. Artificial vesicles that contain reconstituted and functional proteins can also be produced. This increases the complexity of the vesicle preparation process, but also the functionality of the resulting membranes. A last 
option is to use microsomal vesicles, formed from the endoplasmic reticulum during cell disruption. The composition of these vesicles mimics that of real cell membranes, and so do the resulting BLMs. These last types of vesicles allow for the study of more complex and asymmetrical membranes that better mimic real cell membrane compositions [29].

The first step in the membrane creation consists of trapping the vesicle on the aperture by employing a negative pressure [30-31], by applying an attracting dielectrophoretic force [32] or by maneuvering it to the aperture using optical tweezers [33]. After being trapped for a short while, the vesicle bursts to give rise to a planar bilayer structure. As vesicles are solvent-free bilayers, membranes obtained with this technique are also solvent-free. Furthermore, additional flexibility concerning the membrane composition is obtained as the composition of the vesicles can easily be adapted and proteins can be added to them.

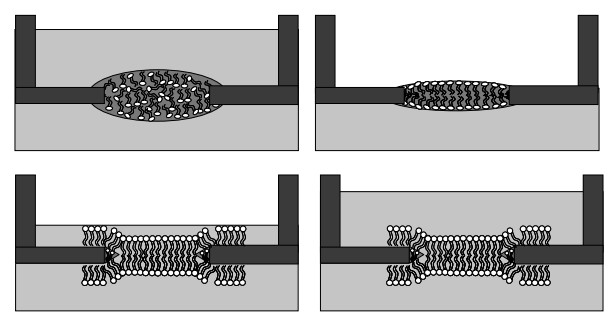

Figure 3.8. Air exposure technique.

Top: Formation of the lipid plug in the aperture and its subsequent exposure to the air. Bottom, left: Upon solvent evaporation, the lipid plug starts to thin automatically, forming a bilayer structure in the middle of the aperture. Bottom, right: To stop further bilayer formation, fresh buffer is added to the top well when the bilayer area fills $\sim 80 \%$ of the aperture.
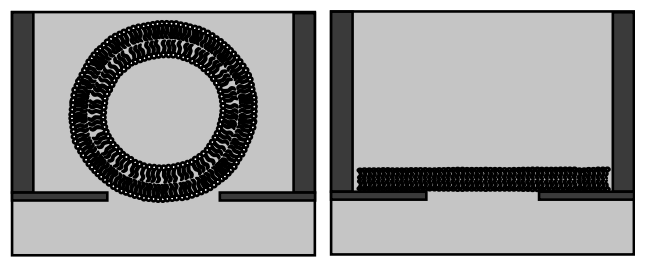

Figure 3.9. Vesicle fusion technique.

A vesicle that is brought in contact with the substrate containing the aperture (left) (by either a dielectric force, optical tweezers or the application of a suction force) will burst either after the application of an external force, such as a suction force, or spontaneously. This results in the formation of a bilayer across the aperture (right).

\section{(v) Droplet interfacing}

A last technique described here consists of forming a bilayer at the interface between two aqueous droplets coated with a stabilizing monolayer of phospholipids and immersed in an organic environment (see figure 3.10) [34-36]. The droplets are either formed by injecting drops of an aqueous solution in an organic-phospholipid solution, or by injecting drops of aqueous solution containing vesicles into an organic solvent (see figure 3.10). In the latter case, different phospholipids can be used for the different droplets, offering the possibility to form asymmetrical membranes. Droplets can be created either manually or in a microfluidic device [36-39]. In the latter case, droplets are generated at the junction between an oil-lipid flow and an aqueous flow. This allows for automatic and large-scale production of droplets having a uniform size. After the droplet formation, the phospholipids self-assemble at the interface between the organic and the aqueous solution. The coated droplets are next moved towards each other until they touch and the bilayer is 
formed (see figure 3.10). Protein can be incorporated into these bilayers for instance by dissolving them in the aqueous solution inside the droplets [35]; after bilayer formation spontaneous insertions occur.

The movement of the droplets is controlled, either manually by placing electrodes in each droplets and moving these together using micromanipulators [35], by a dielectrophoretic force [34] or due to a fluidic flow [36]. Electrical measurements are possible after insertion of electrodes in the droplets, as the oil forms an insulating layer around the droplets, which makes experimentation on the BLMs less straightforward as for the membranes suspended across an aperture. However, a large advantage of this technique is the increased stability of the membranes from a few hours to a few days [35]. Furthermore, it is possible to separate the droplets after they have formed a bilayer, considerably increasing the flexibility of this technique $[35,40]$. One single moving droplet, containing a specific type of proteins, can, for instance, be scanned against several static droplets that have a different buffer solution inside, giving information about the proteins response to the different buffers. With this technique, it is also possible to create networks of multiple membranes by forming straight or branched chains of multiple droplets [40]. Such networks are for instance employed to study the communication between different droplets through bilayer-incorporated proteins [35].

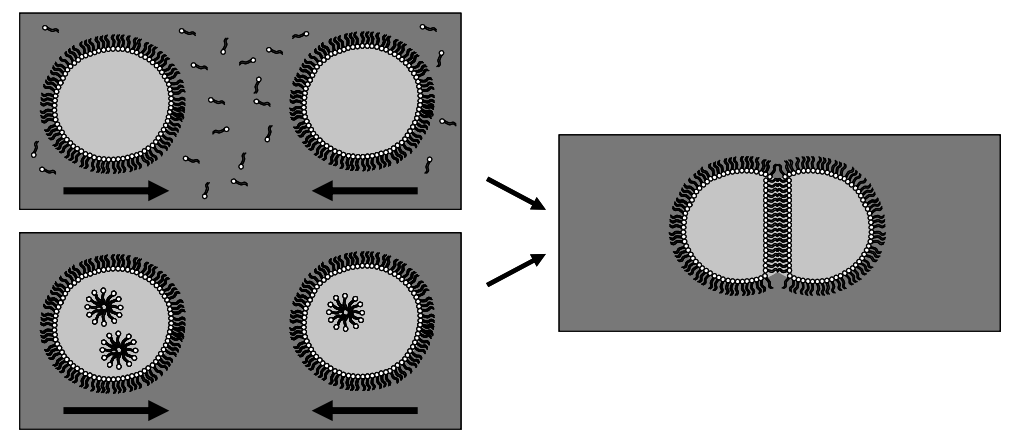

Figure 3.10. The droplet interfacing technique.

Left: After the creation of two aqueous droplets in an organic solvent, phospholipids self-assemble into a monolayer at the interface between the two buffers. Right: When these two droplets are brought in contact with each other, a phospholipid bilayer forms in the area where they touch each other.

\section{(vi) Advantages/disadvantages of the BLM preparation techniques}

The most straightforward technique to produce bilayer lipid membranes is the MuellerRudin technique as it does not involve any (complex) fluid handling or the production of vesicles. Nevertheless, these membranes always contain solvent, both between the leaflets and in the annulus region. Therefore, the characteristics of the membranes, such as their thickness or their surface area, are ill-controlled and ill-reproducible. Furthermore, with this technique only symmetric membranes can be formed. As a cell membrane has an 
asymmetrical composition, other techniques that lend themselves better to the production of asymmetrical membranes, such as the Montal-Mueller, the tip-dip or the vesicle fusion technique, provide more realistic experimentation platforms when looking into cell membrane properties. On other aspects, the droplet interfacing technique results in the most flexible approach with respect to membrane composition, as it allows for fast changes in the leaflet structure and in the bilayer networks.

\section{Protein integration techniques}

A large amount of BLM experimentations employ membrane proteins for drug screening purposes or for investigating their functioning. However, the insertion of proteins in BLMs is not always straightforward, and requires different strategies depending on the nature of the proteins and their "solubility".

In the most favorable case, the proteins are soluble in aqueous buffers. They are simply expressed in bacteria, recovered from the bacterial lysate, and frozen as a powder. This powder is solubilized for further experimentation. Peptides are either isolated in the same way as the proteins or synthesized. Most of the time, the proteins or peptides are added to the buffer in one of the reservoirs and they incorporate spontaneously in the membrane when a small pressure is applied or the solution is stirred [41-42]. Alternatively, peptides can be dissolved in the phospholipid-solvent mixture used to prepare the membranes, and are thereby directly included in the BLM

Unfortunately, most of the membrane species are not soluble in water as they contain a hydrophobic part and they must be preserved in a membrane-like environment. In that case, they are extracted from bacteria in microsomes from the endoplasmic reticulum (ER). Subsequently, two strategies are conceivable for their insertion in a BLM. Firstly, the microsomes are directly fused with the BLM, as described in the article from Miller and Racker [43]. Alternatively, the microsomes are re-suspended in a solution containing the detergent Triton X-100 [44], so that the proteins are isolated in micelles. Following this, phospholipids are added to the resulting solution in order to reconstitute the proteins in the vesicles. The detergent is subsequently removed and absorbed in porous beads [44-45], and the resulting artificial vesicle solution employed for BLM experimentation. To fuse these vesicles with horizontal membranes, the vesicles containing the proteins are brought into contact with the membrane by simply letting them "sink" into the bilayer surface where they spontaneously fuse. For their fusion with vertical membranes a salt gradient is created across the membrane, from the trans- to the cis-compartment, and the diffusion of the vesicles in the cis-compartment to(wards) the membrane is promoted by stirring the solution. As soon as a vesicle approaches the membrane, water is transported from the transcompartment into the vesicle as a result of the salt gradient across the membrane, causing it to swell, stretch and eventually fuse with the BLM [46]. Vesicle fusion can be monitored by adding in the membrane of the vesicles the antibiotic nystatin and ergosterol (a plant sterol species) that form a pore together if the concentration of ergosterol is large enough [47]. 
When the vesicle fuses with the ergosterol-free membrane, the conductance of this pore can be measured briefly across the BLM, indicating successful vesicle fusion. However, in the planar bilayer, the nystatin-ergosterol complex quickly dissociates and has little chance to form again as their concentration is too low, allowing measurements only on the proteins of interest [47-48]. After insertion of the protein in the membrane, the salt concentration is equilibrated on both sides of the membrane, since a salt gradient across the membrane results in the formation of a trans-membrane potential.

A last technique to insert proteins in the membrane relies on their transfer from bacterial colonies into a BLM. For this purpose, a sharp glass probe is placed into contact with the bacterial membrane to isolate the proteins and subsequently brought into contact with the BLM to allow the proteins to insert in the BLM [49]. This last technique is employed the least often, as it involves extensive micromanipulation to bring the probe into contact with both membranes.

\section{E. BLM applications and related techniques}

BLMs are mostly used for two different types of applications: (i) fundamental investigations on membrane properties and (ii) experiments employing membrane proteins for sensing and drug screening purposes, for instance. In the latter case, the BLM is acting as a matrix for the proteins. The following sections illustrate these two main classes of experiments.

\section{(i) Fundamental studies on membrane properties}

The overall goal of fundamental studies on membranes is to establish a relationship between the composition of the membrane and its properties. Motivations for these fundamental studies are manifold as elucidating this composition-property relationship is a first step towards understanding how a cell membrane functions and regulates its properties. With this knowledge, techniques to pass the cell membrane, can be developed. An example of this is the topic of this thesis, electroporation, which has been discussed in detail in the previous chapter. The formation of pores in the membrane due to the application of an electric field greatly depends on its precise composition $[12,50]$. By better understanding the molecular interactions in the membrane and the steps involved in the pore formation process, improved protocols can be designed to enhance both the efficiency of electroporation and the cell survival rate. Crucial and interesting properties of a cell membrane are (i) its thickness, (ii) the packing density of the phospholipids (and the resulting fluidity of the membrane) and (iii) its possible curvature. These characteristics of the membrane directly correlate to (i) its phospholipid content via the nature of both the phospholipid head groups (i.e. glycero- vs. sphingo- vs. glycolipids) and the hydrocarbon chains (i.e. length and presence/number of unsaturations); (ii) the cholesterol content; and (iii) to the presence of proteins. Consequently, an alteration in the membrane composition directly affects its structure.

The membrane composition can be altered due to external or internal stimuli. For 
instance, when a cell becomes sick, intracellular changes occur that lead to a change in the membrane constitution. In the case of cancer, it is now well-established that the phospholipid and cholesterol compositions of the plasma membrane are altered compared to healthy cells [51], and this is discussed in more detail in chapter 6 of this thesis. This implies that the membrane behavior e.g., its barrier properties, is likely to be modified. Recently, a clear relationship between an external influence, i.e. the diet, and the cell membrane composition has led to a question about the impact of nutrition on the cell membrane properties [52-53]. Besides, the membrane composition and properties, on one side, and the functioning of peptides and proteins, on the other side, have a mutual influence on each other. This is particularly illustrated with the small peptide gramicidin A: its pore-forming ability is lowered in case of a thicker membrane [13] and its presence in a membrane influences the properties of the membrane by locally modulating the membrane thickness [54-55]. The presence of small-sized domains, or so-called lipid rafts, in membranes is another important property of membranes, which is currently generating a lot of interest and excitation. Depending on the precise ratio between different phospholipids and cholesterol, the lipids may separate into different domains, according to their affinity for each other. These domains are thought to play a major role in modulating the rigidity of the membranes, and to be involved in various cell signaling processes [56] as they host specific membrane proteins that cluster in the domains. Furthermore, raft proteins might display an altered behavior as they are located in a changed lipidic environment.

To study the membrane properties a great variety of techniques can be employed: structural analysis techniques, optical techniques, mechanical techniques, electrical techniques and molecular dynamics simulations (MDS). The choice of the most suitable technique(s) is most often motivated by the properties that are examined.

Structural analysis techniques, such as nuclear magnetic resonance (NMR), neutron scattering and X-ray diffraction enable to determine the thickness of the membrane, the ordering of the phospholipid tails, the packing density and the conformation of the molecules in the membranes. While most of these methods require cooling of the membrane to slow down or stop the motion of the molecules, NMR analysis can be performed at different temperatures. This provides additional information on the mobility of individual molecules in the membrane, which correlates to its phase composition. For instance, NMR studies have enabled to show the dependency of the membrane phase composition on both the temperature and its cholesterol content [57-58].

Optical techniques are the most employed for BLM experimentation, although not for planar (vertical) BLMs but for vesicles. Despite the fact that this chapter mainly discusses planar BLMs, this class of techniques is still reviewed here as the novel trend of miniaturization enables horizontal membrane preparation on which optical studies become possible. Regular microscopy has a size-dependent detection limit (the optical resolution is $0.2 \mu \mathrm{m}$ ) so that only relatively large structures, compared to the size of the lipids and 
proteins, can be observed, such as domains larger than $0.2 \mu \mathrm{m}$. The detection of the phase composition of the membrane and the presence of domains relies on the use of fluorescent phospholipids or markers that specifically reside in certain phases [57, 59-60]. When looking directly at the fluorescent emission of these markers, the existence of different phases and/or domains gives rise to specific fluorescent patterns. The motion of lipids can be studied by related techniques, such as FRAP. From this, the nature of the phospholipid phase as well as their related packing density can be extrapolated [61-63]. Last, fluorescence techniques have been applied to visualize the formation of pores in a membrane, both in the lipid matrix or due to the insertion of a pore-forming entity such as $\alpha$-synuclein or $\alpha$-hemolysin [33, 64]. Pore formation is detected here by the leakage of a dye introduced on one side of the membrane. Although optical techniques are dominating in the field of vesicle experimentation, the addition of fluorophores in the membrane changes its properties and subsequently may affect the experimental outcome [60]. Furthermore, since the optical resolution is limited to $0.2 \mu \mathrm{m}$, the observation of nanometer-sized structures is precluded. Nevertheless, optical techniques still hold great value, not only due to their relative ease of application but also by the multitude of markers available to show different membrane constituents and properties.

The third class of techniques, mechanical techniques, makes use of force measurements to determine indirectly membrane properties. A mechanical property which is widely investigated is the compressibility of a lipid monolayer on a water surface as this directly correlates with the phase composition of the membrane [7]. The compressibility of the monolayer is studied in a Langmuir through by changing the area of the monolayer and measuring the lateral pressure [7]. However, the currently most popular technique to study mechanical properties of membranes is certainly AFM. This scanning technique is widely employed to study the rigidity and the deformability of membranes, and its nanometer lateral and axial resolutions make it very attractive to detect and characterize nano-domains [65-67]. However, AFM is not suitable to scan membranes suspended across conventional apertures having a size $>50 \mu \mathrm{m}$, as the deflection of the membrane affects the experimental outcome. Consequently, supported membranes must be employed [65-67] or, alternatively, membranes suspended on nm-scale apertures [68]. AFM-based experimentation is also employed to measure the force required to push the AFM tip through a bilayer, as this gives information about the mechanical resistance as a function of the membrane composition. This, in turn, provides indirect information on the packing density of the phospholipids and the phase composition of the membrane [69].

The next group of techniques relies on electrical measurements, i.e. on the detection of a leakage current through a membrane. Electrical techniques are particularly appropriate for BLM experimentation as both sides of the membrane are easily accessible for the introduction of electrodes (which is not the case for vesicles and cells). The most frequently used electrical characterization method consists of measuring possible leakage currents through the membrane. Firstly, this allows for monitoring the formation of the BLM and 
measuring its seal with the substrate. Secondly, the incorporation of pore structures (e.g. $\alpha$ hemolysin) can be followed in real-time as each protein insertion gives rise to a specific leakage current. Similarly, the ability of peptides such as alamethicin and gramicidin A or D to form pores as a function of the membrane composition can be elucidated. Both peptides are freely floating monomers and form transient pores upon dimerization. This process of dimerization is affected by the composition of the membrane, as already mentioned; when the membrane is thick, for instance if the chain length of the phospholipids is long, the two monomers cannot contact each other, preventing thereby the formation of a pore [13]. Thirdly, leakage current measurements are employed to investigate the process of pore formation during electroporation, i.e. upon application of an electric field on a membrane. Not only the size and the stability of the pore but also the effect of the membrane constituents on the probability of pore formation can be determined using electrical measurements (see chapter 2). We have demonstrated, for instance, that the glycerolipid composition has little effect on the process of pore formation. However, the addition of sphingolipids or cholesterol dramatically changes the behavior of the membrane. Cholesterol has a bidirectional effect depending on both the nature of the phospholipid used and its concentration [12, 14, 70]. Moreover, the addition of sphingolipids to membranes containing glycerolipids and cholesterol may lead to the formation of domains in the membranes. The formation of those domains can have both a destabilizing and a strengthening effect on the membrane either caused by phospholipid packing problems at the edge of these areas or by the condensed phospholipid density in the domains, respectively [50]. The presence of pore-forming entities such as gramicidin D [55] or prestin [71] results in a stepwise and concentration-dependent increase of the membrane resistance. These findings have been confirmed in the case of gramicidin D by MDS: the peptide locally changes the thickness of the membrane, and in doing so, reduces the chance for pore formation [54-55]. On other aspects, the electrical characterization of membranes provides indirect information on the membrane properties such as its thickness, the phospholipid packing density and the phase composition of the membrane [70], as these different parameters influence the process of pore formation [72-74].

A last approach for fundamental studies on BLMs relies on computation, and consists of MDS. MDS provide a powerful toolbox to study molecular interactions in membranes and determine how these interactions may affect the membrane properties. The goal of MDS is obviously to describe in detail the membrane, in a dynamic way [75]. Since a membrane is a very complex structure with very intricate interactions, simplification of the situation is often required. However, computers have become powerful enough nowadays to enable a comparison between simulated situations and experiments. Membrane simulations require (i) a molecular model that describes the interactions taking place in the membrane at the molecular or atomic level and (ii) computational techniques to calculate the overall properties of the membranes (such as the thickness, the phospholipid organization or orientation) [72]. Three different classes of molecular models are dominating for MDS [72]. 
Firstly, the so-called all-atom models take into account every single atom in the membrane model. While this approach is the most precise and complete, it requires extensive computational power to model a small piece of membrane ( 5000 lipid molecules) for a very short time period (in the order of $\mu$ s ) [72], and this reduces its applicability. To increase both the membrane area that can be simulated and the duration of the simulation, the molecular models must be simplified. Less complicated approaches employ for instance united-atom models or coarse grain models (CG). In the former approach, aliphatic carbons and their associated hydrogens, for instance, are modeled into one particle, while for the latter the building blocks are even larger molecular units. CG models can be so "simple" that they only capture the essential elements of the molecules; e.g., a phospholipid is represented by a chain of three particles, one for each tail and one for the head group, with different interaction strengths between them. However, CG models can also be more detailed, consisting of more separate units, to possibly distinguish between different phospholipids.

The major selling point of MDS is that all parameters can be controlled and changed, which is not possible during experimental studies, resulting in an unlimited flexibility in situations that can be simulated. They can not only provide any type of information on the membrane properties, for instance on the membrane thickness or the interaction between the different phospholipids (phase diagram / raft formation) [76-79], but also about the response of a membrane to external stimuli such as an electric field or mechanical stress [80]. To summarize, MDS are a strong tool to study molecular interactions that are involved in changing the properties of BLMs in detail. Since computing power is still increasing, the use and application of these simulations will only grow.

\section{(ii) BLMs as platforms for protein-based experimentation}

Although basic knowledge on membrane properties is primordial to understand how a cell can protect itself from its environment, fundamental studies do not represent the main class of BLM experimentation. The primary application of membrane models is their use as platforms for protein-based experiments, and this includes three categories of studies: (i) drug screening on membrane proteins, and especially ion channels (ii) fundamental and engineering studies on channel proteins, and (iii) the use of ion channels (primarily $\alpha$ hemolysin) as nanopores for (fundamental) biophysical analysis. In the following paragraphs, these three types of experiments are reviewed in more detail.

Miniaturization of BLM experimentation is mainly driven nowadays by the development of "high-throughput" drug screening platforms on membrane proteins. The latter have indeed become preferred targets for the development of new drugs, and now represent about $50 \%$ of the targets for drug design. Firstly, membrane proteins are sitting in the membrane; they are therefore easily accessible. Secondly, they have a crucial role in the regulation of the cell functioning: they are involved in the trans-membrane transport and in the communication of the cell with its environment and they often (de)activate intracellular signaling pathways. The disturbance of the function of ion channels, either due to a 
mutation or by autoimmune attacks, is consequently often correlated to diseases, so-called channelopathies [81]. One example of channelopathies is cystic fibrosis which is caused by a dysfunctioning chloride channel (cystic fibrosis transmembrane conductance regulator, CFTR) [81]. For these screening applications, dysfunctioning ion channels are incorporated into BLMs, and the effect of different medicines on their channel properties can be monitored by looking at the leakage current through the membrane.

The second class of protein-based applications focuses either on getting a better understanding the channeling properties of membrane proteins or on tuning these properties by protein engineering. The ion conductivity properties of a protein channel include the nature of the ions that possibly pass through the pore, the direction of the ion flow, and the rate of the ion flow. These properties are affected, for instance, by the composition of the buffer solution, its ionic strength and $\mathrm{pH}$ [82-84] or they can be modulated upon addition of chemicals (partially) blocking the pore. For example, polyvalent cations or dextrans are shown to transiently block the pore of $\alpha$-hemolysin [8486]. Alternatively, the channel properties can be altered or "tailored" by engineering the protein and mutating specific amino-acids [15]. Mutated or engineered proteins can display a changed behavior depending on the location of the mutation(s); they do not conduct certain ions, or open or close upon a specific external trigger. BLM provides a platform for screening the mutated proteins and identify the impact of certain mutations on the functioning of the proteins. By engineering $\mathrm{OmpF}$ (outer membrane protein $\mathrm{F}$, a nonselective porin molecules found in the outer membrane of Escherichia coli), Miedema et al. successfully modified its behavior from non-rectifying (resistance-like behavior) to rectifying (diode-like behavior) [87-88]. Furthermore, Koçer et al. modified the switching behavior of MscL (mechanosensitive channel of large conductance) from Escherichia coli by attaching photosensitive modulators; the modified channel opens when exposed to light and not anymore to a mechanical stimulus [89].

The last application discussed in this section is the use of proteins or peptides that are always in the open state, and primarily of $\alpha$-hemolysin, as an alternative to solid-state nanopores for analysis purposes and fundamental biophysical studies on DNA (Deoxyribonucleic acid) [90]. $\alpha$-Hemolysin has a very well defined pore diameter and the formation of a BLM containing an $\alpha$-hemolysin pore does not require complex fabrication processes, as is the case for artificially manufactured nanopores [91-92]. Studies of DNA with $\alpha$-hemolysin rely of the fact that the negatively charged DNA is forced through the pore upon application of a voltage across the membrane [93]. A first "holy grail" for these biophysical studies is the possibility to sequence DNA molecules. As each base interacts differently with the nanopore, a base-specific decrease in the amount of current through the pore is expected [93]. The measured signal gives in theory the sequence of the ssDNA (single-stranded DNA) molecule. Unfortunately, it remains difficult to distinguish reliably between the different bases using $\alpha$-hemolysin nanopores, and no successful sequencing on whole DNA has been performed using this approach. Similarly, $\alpha$-hemolysin has been 
applied for polymer analysis, not only in terms of the nature of the polymer but also regarding its length. Polymers with different chemical natures give different amounts of blockade of the current, depending on their interaction with the $\alpha$-hemolysin pore, while the time duration of the blockade is directly related to the length of the polymer. [93-94]. Next to these analytical applications, $\alpha$-hemolysin is also widely employed for more fundamental and biophysical studies on DNA molecules, for instance for uncoiling ssDNA and unzipping dsDNA (double-stranded DNA). The pore of $\alpha$-hemolysin is wide enough to transport ssDNA but not dsDNA. By studying the translocation times of ssDNA through the pore of $\alpha$-hemolysin, the dynamics of the uncoiling process and the length of the piece of DNA can be determined [95-96]. Furthermore, the amount of force required to unzip dsDNA and the residential and translocation times as a function of that force gives information about the interaction strength between the two strands of DNA [97].

To summarize, experimentations using BLMs are very versatile, and concern many different fields of applications. On one hand, fundamental studies provide increased knowledge about the structure and the functioning of cell membranes. This is of prime importance to understand better the response of cells to certain (external) stimuli, such as the effect of an electric field, diseases or diet. On the other hand, BLMs are increasingly employed as platforms for encapsulating membrane proteins. In this category, the two leading applications are the use of $\alpha$-hemolysin as a natural nanopore and the development of "high-throughput" platforms for drug screening assays. By miniaturizing the BLM devices, matrices of BLMs can be realized, that allow for the simultaneous recording on multiple proteins. Furthermore, by integrating such a matrix with microfluidics, the resulting multiplexed tools move towards the dream of having artificial and reliable experimentation platforms for "high-throughput" screening of proteins [98-100]. In this context, especially multiplexed protein assays for drug screening applications and DNA sequencing are evolving rapidly to become research tools for pharmaceutical purposes. The introduction of such devices will decrease the time required to perform screening assays, considerably reducing the development time and related cost for new medicine and genome determination. The development in the direction of these devices is discussed in the next section of this chapter.

\section{Miniaturization and integration with microfluidics}

The use of miniaturized and microfluidic devices for BLM experimentation has increased in popularity due to the numerous advantages they bring. In the coming section, first the motivations for both the miniaturization of BLM experimentation and its combination with microfluidics are discussed. Following this, an overview of existing miniaturized and microfluidic devices dedicated to BLM experimentation is given. 


\section{A. Motivation for the miniaturization of BLM experimentation and its integration in microfuidic platforms}

BLM experimentation can benefit greatly from its miniaturization and its combination with microfluidics. Both approaches present distinct advantages, for specific aspects of BLM experimentation. We firstly describe the trend in miniaturization especially in the frame of microfluidic applications as well as the general advantages found in miniaturized and microfluidic devices. Following this, the two levels of miniaturization of BLMs, on one hand, and their implementation in a microfluidic platform, on the other hand, are presented and the distinct advantages brought by the two approaches discussed.

\section{(i) Miniaturization and microfluidics}

The field of microfluidics started with the miniaturization of separation tools such as gas chromatography (GC) and capillary electrophoresis (CE) [101] down to the micrometerscale, and this still represents one of the key-applications of microfluidic devices. For these applications, the driving forces for the miniaturization are the gains in separation time, separation quality and sensitivity while using portable devices exhibiting an overall reduced consumption. This led to the emergence of the new field of Lab-on-a-Chip (LOC) technology. LOC devices are defined as systems with a size of a few $\mathrm{cm}^{2}$ that present internal structures with typical dimensions of 10-100 $\mu \mathrm{m}$. These devices are either produced using microtechnology techniques in glass or silicon or machined in polymer materials using emerging techniques or standard processes from the polymer industry [102]. Since their early development, LOC devices have seen their applications diversified and extended to the fields of, among others, sensors, analysis, chemical synthesis and reactions, cell analysis, cell biology and tissue engineering [103].

LOC devices present a number of general advantages compared to conventional labscale instrumentation. A first series of advantages is linked to their small size. LOC devices are portable, which is of great interest for on-site environmental and point-of-care analysis. Although they are still, in general, coupled to standard bulky labware, completely portable devices that integrate everything, from sampling to detection to communicating the results to the user are emerging (for instance, Medimate Multireader ${ }^{\circledR}$, Medimate B.V., Enschede, The Netherlands). On other aspects, for environmental and medical analysis, the capacity of the system is comparable to the amount of sample usually available (low $\mu \mathrm{L}$ range) so that the analysis can proceed directly without a first dilution of the sample. Small-sized devices also bring faster processes especially for separation and diffusion-limited processes as molecular diffusion scales with the square of the internal dimension of the device. Similarly, heat exchange is promoted, and highly exothermic processes can safely be carried out without any risk of explosion. In addition to this, the overall consumption (and waste production) is highly reduced, not only in terms of reagents and solvents but also in terms of energy. Finally, these reduced distances and volumes found in microfluidic devices give specific flow patterns and a laminar flow profile, which brings novel experimental 
possibilities [101]. Flows are better controlled at the microscale and liquids are easily and quickly replaced in micrometer-sized channels, making the experimental conditions highly dynamic.

Another series of advantages of microfluidic systems over traditional systems originates from the processes employed for their production. LOC devices fabricated with microtechnology techniques exhibit a high level of reproducibility, and this in turns guarantees the repeatability of the processes carried out in the devices. On other aspects, LOC devices are produced using batch fabrication techniques (both microtechnology techniques and standard polymer processes), which greatly reduces the fabrication costs. Finally, LOC devices present a high level of integration; they can integrate on a single device not only a large number of identical components (horizontal integration) but also several steps of a whole analytical process (vertical integration). Finally, LOC devices are easily connected to dedicated interfaces for automation of processes, i.e. liquid flows and the measurements, facilitating high throughput assays.

\section{(ii) Miniaturization of BLM experimentation}

The first step of miniaturization of BLM experimentation is limited to downscaling the aperture and/or the fluidic reservoirs. The realization of miniaturized apertures down to a few tens of nanometers with a high level of reliability and reproducibility is made possible using micro- and nanofabrication techniques. Miniaturized apertures not only bring about more stable membranes with a lifetime of up to a few days instead of a few hours [3] but also a reduction in the electrical noise. This is accounted for by an increase in the seal resistance to hundreds of $\mathrm{G} \Omega$ and a reduction in the membrane capacitance to several $\mathrm{pF}$, or even below since the surface area is very small [31, 104-107]. Consequently, experiments on the membranes can be performed for a longer period of time and the enhanced electrical detection scheme favors sensitive recording, which is especially important when studying the activity of single ion channels [108]. On other aspects, smaller apertures also increase the likelihood for single protein studies, since the surface area in which proteins insert is reduced. Finally, the BLMs created in apertures with a $1-10 \mu \mathrm{m}$ diameter have a size comparable to that of a whole cell membrane, and those with an even smaller aperture dimension to a section of a membrane; this provides a more relevant experimental environment for membrane studies.

On other aspects, miniaturized apertures produced using microfabrication techniques can easily be created as aperture arrays towards parallelized and simultaneous analysis on as series of membranes. However, the issue here is to be able to develop proper fluidical and electrical connections to address the membranes individually [109-110].

The other feature of the miniaturization is the reduction in size of the fluidic reservoirs. In this case, the devices can contain one or two microliter-sized fluidic wells. The smaller volume of these reservoirs allows for faster perfusion or replacement of the solutions, and gives shorter diffusion times for the molecules in solution (such as proteins or chemicals) to 
reach the membrane. The combination of these two factors result in faster experiments, increasing not only the amount of assays that can be performed in a certain period of time, but also the likelihood for a successful outcome (since the longer an experiment takes, the higher the probability for rupture of the membrane). Furthermore, downscaling the reservoir also increases the sensitivity of the electrical measurements [94]; the stray capacitance existing between the electrodes and the electrolyte depends on the volume of electrolyte: the larger the volume the higher the current noise.

\section{(iii) Implementation of BLM experimentation in a microfluidic device}

The second level of miniaturization consists of implementing the BLM experimentation onto a LOC device. In that case, the aperture is miniaturized, the fluidic reservoirs are replaced by one or two microfluidic channels (as detailed below), and electrodes, as well as other fluidic components can be integrated into the same device. Microfluidic devices for BLM experimentation globally presents the same advantages as mentioned previously for microfluidic devices in general. Still, additional advantages specific to this type of applications are found. Firstly, perfusion of solutions in the fluidic reservoirs is facilitated in a microfluidic environment and dynamic experiments become possible. This opens the route to novel types of experimentation such as the transient exposure of the membrane to a given flow, as would occur in vivo. Thereby, the response of both cell membranes and specific proteins to a shear stress can be investigated. A key-feature of microfluidic devices is their high level of integration. Aperture arrays produced using microfabrication techniques are easier to address individually when combined with microfluidic networks towards multiplexed experiments [100, 111]. The electrodes can be integrated close to the apertures and fabricated using microtechnology techniques such as the deposition of metals using evaporation techniques and patterning of the resulting metal layer using etching techniques [112]. This contributes to the automation of the experimentation [32, 112-113], which, as explained in section IEii, is the future for many bilayer assays. Furthermore, the microfluidic platform can also include other fluidic components, as discussed in more details in chapter 7. The lipid mixtures can be generated ,for instance, on the chip, and the membrane preparation can easily be automated in a LOC environment. When the experimentation is automated in a multiplexed platform, "high-throughput" studies are possible, for instance, for screening a number of experimental parameters in a single assay. This aspect is particularly relevant for pharmaceutical applications of BLMs, i.e. the high throughput screening (HTS) of drugs on membrane proteins, as discussed in section IEii of this chapter.

However, the main advantage of microfluidic platform for BLM experimentation is found in the range of characterization techniques available for the BLMs. Using a conventional set-up, BLMs are vertical and their study is mainly restricted to electrical techniques. On the contrary, in microfluidic devices, membranes are mostly horizontal and the fluidic reservoir under the membrane is "shallow" $(<100 \mu \mathrm{m}$ height). Consequently, optical techniques are routinely coupled to the electrical measurements towards a more 
complete characterization of the BLMs. In addition to this, if the fluidic reservoir located above the membrane is not a microfluidic channel but an open well, other characterization techniques are conceivable such as scanning techniques (for instance, atomic force microscopy (AFM), scanning ion conductance microscopy (SICM) and scanning electrochemical microscopy (SECM)) which can provide nm-scale information on the membranes.

\section{B. Different levels of miniaturization and micro-fluidic integration}

In the following section an overview is given of miniaturized and microfluidic platforms for BLM experimentation, and this overview is restricted to suspended BLMs. The devices are classified in three categories: (i) devices that include miniaturized apertures and/or fluidic reservoirs, (ii) half-microfluidic devices where only one fluidic reservoir is replaced by a microfluidic channel and (iii) full microfluidic devices where both fluidic reservoirs consist of microfluidic channels. The three categories are considered successively in the three following sections.

\section{(i) Miniaturized conventional systems}

The first step towards miniaturization concerns the aperture and/or the reservoirs, although fewer examples are found for this second aspect. In the first case, the aperture size lies in the micro- down to the nanometer-range. Small and well-defined apertures are produced using micro- or nanofabrication processes in a great variety of substrates and with different fabrication processes, as reviewed in table 3.1. The fabrication process is dictated by the nature of the substrate in which the orifices are made as well as their final size, as reviewed below. In this category of miniaturized devices, the substrates containing the apertures are fixed between two conventional $\mathrm{mL}$ - or $\mu \mathrm{L}$-sized reservoirs usually made from polymers such as Teflon [3, 114] or PDMS (polydimethylsiloxane) [32].

One of the first reports on the microfabrication of an aperture for BLM preparation employs a membrane made from polyimide suspended on an oxidized silicon substrate (see figure 3.11A) [3]. The thin layer (6- $\mu$ m thickness) of polyimide contains a $50-\mu \mathrm{m}$ aperture produced using photolithography. Thereafter, the back of the silicon wafer is etched using wet-etching techniques to result in a suspended polyimide film on the silicon substrate, with the aperture accessible for measurements. The resulting device is clamped between two Teflon reservoirs (volume in the order of several $\mathrm{mL}$ ) and the Montal-Mueller technique is employed to prepare the BLMs. For the first time, membranes with a very long lifetime (>50 h) are reported with a high electric seal of 20-30 G $\Omega$. In addition to this, the device performance is illustrated with the insertion of pore-forming species such as the voltagegated pore alamethicin and the ligand-gated ion channel $\mathrm{nAChR}$ (nicotinic acetylcholine receptor) and the recording of their electrical activity.

Not surprisingly, the most employed material for miniaturized apertures is silicon, as 
shown in table 3.1, and this in different forms: as "bulk" silicon, silicon-based materials (such as silicon dioxide or silicon nitride) or even "porous" silicon. The popularity of these materials is easily explained by the fact that technologies employed to process them are amongst the most mature in the field of micro- and nanofabrication, and that low-micronsized structures can easily be produced in these materials. Different processing techniques are reported to fabricate the aperture depending on the nature of the substrate employed to make the membrane, as detailed in table 3.1. Amongst them, ion-milling [107] and electronbeam lithography $[107,113]$ enable the fabrication of the smallest aperture, with a minimal size of $30 \mathrm{~nm}[107,113]$, as opposed to the micrometer-sized orifices produced by laser drilling techniques and the most popular etching techniques [32, 107-108, 112, 114-117].

However, silicon itself is a semi-conductive material, which precludes any electrical measurements so that the device must comprise an insulating material. Consequently, in most of the cases, an insulating (silicon-based) material is directly employed to make the membrane that contains the aperture. To achieve this, an additional layer of insulating material must be deposited on the silicon membrane, either by thermal growth (silicon dioxide) [112, 114-117] or using plasma enhanced chemical vapor deposition (PECVD) (silicon nitride) [32, 107-108, 113].

On other aspects, most of the devices contain a free-standing membrane, as shown in figure 3.1. Such a structure is characterized by a large capacitance that deteriorates the sensitivity of the electrical measurements. Consequently, an additional (thick) layer of insulating material is required to restore the quality of the electrical measurements, and SU$8[112,116-117]$ or polyimide [108] are commonly employed for that purpose. A final step consists of coating the substrate around the aperture with a hydrophobic material, such as a fluorinated polymer [112, 114, 116-117] to promote sealing of the BLM on the substrate (seal resistance $>20 \mathrm{G} \Omega$ ) and subsequently to enhance the quality of the electrical measurements. Figure 3.11B shows one example of such a multiple-layer device.

A more straightforward option consists of directly employing insulating materials such as glass or fluorinated polymers like Teflon (the standard material for conventional BLM setup) and polyvinylidene fluoride (PVDF). In that case, the device is fabricated in a single step and comprises of a single substrate (see table 3.1). Both glass and Teflon are also transparent, enabling optical measurements. In glass, apertures in the micrometer-range are formed by standard wet-etching [31, 104-105] or laser drilling techniques [107]. On other aspects, Teflon is mostly used as commercially available Teflon films, and apertures are conventionally formed by non-microtechnological means such as mechanical punching, drilling or spark assisted chemical engraving (SACE) [106]. They have a limited size of $>15 \mu \mathrm{m}$. Smaller apertures (down to $2 \mu \mathrm{m}$ ) are reported when spin-coating liquid Teflon either around a sharp probe tip for the production of a single aperture or on a PDMS master for the formation of an array of pores (see Figure 3.12A) [106]. Alternatively, apertures in PVDF are formed by laser drilling and have smaller diameters, down to $1 \mu \mathrm{m}$ [107]. 

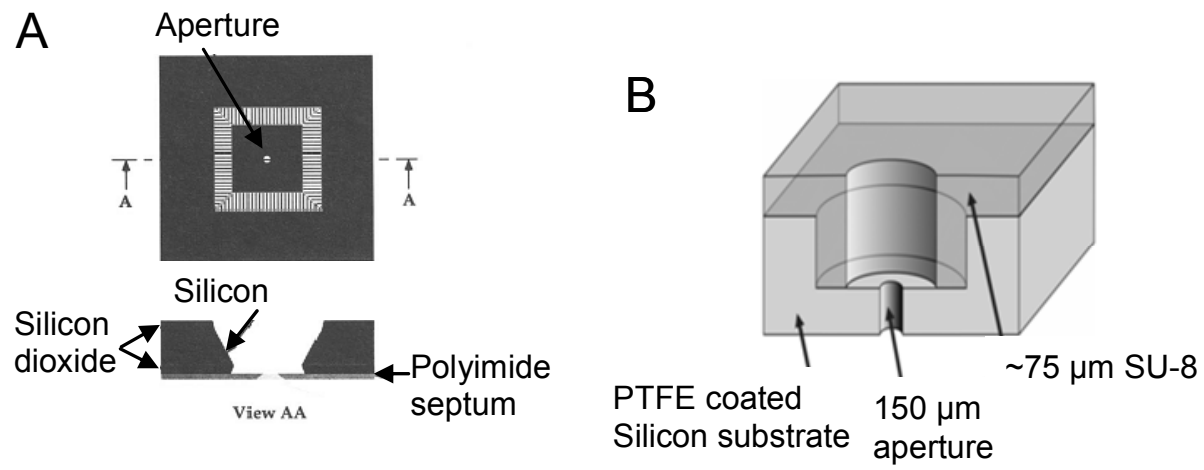

Figure 3.11. Miniaturized devices.

Examples of miniaturized devices including a polyimide- (A) or silicon-based membrane (B). A) A 50- $\mu$ m aperture is produced by photolithography in a 6- $\mu \mathrm{m}$ thick polyimide layer placed a silicon substrate [3]. B) A 150- $\mu \mathrm{m}$ aperture is realized using the DRIE technique in a thinned silicon wafer; a 75- $\mu m$ thick capacitance-reducing SU8 layer is subsequently patterned on the silicon-based membrane and the vicinity of the aperture is coated with Teflon [116].

With most of the mentioned fabrication techniques, arrays of apertures can be fabricated in the silicon, glass and Teflon substrates. However, an alternative approach has been reported by a group to produce large arrays of micro- or nanometer-sized BLMs in silicon and alumina to give "porous" substrates (i.e. with a large number of apertures), as shown in figure 3.12B. The pores (size < $100 \mu \mathrm{m}$ [118]) are created in a silicon substrate by a photolithography step, followed by an anodization process whereas for the alumina substrates only an anodization process is employed and pores are much smaller $(<300 \mathrm{~nm}$ [118-122]). Furthermore, as before, a layer of insulating silicon oxide is added onto the silicon substrate by thermal oxidation. In both cases, the substrate is coated with gold on which a layer of phospholipids is adsorbed to make the surface hydrophilic and promote the formation of a BLM. As a result, the individual membranes are mechanically decoupled; i.e. pore-spanning membranes, formed from the same phospholipid layer, rupture individually instead of simultaneously. The advantage of such porous membrane supports is the prolonged bilayer stability of up to several days and the possibility to perform single channel recording in a large array of membranes simultaneously.

It should be noted that another material is also reported for producing substrates with miniaturized apertures for BLM experimentation, PMMA (polymethylmethacrylate) [123]. PMMA is routinely processed using various techniques such as laser and mechanical machining, but also with nanolithography techniques [102]. Consequently, this material appears to be a good alternative material for the production of nm-sized apertures. 
Table 3.1 presents an overview of the different approaches for miniaturizing the aperture in a conventional set-up. It notably includes: (i) the different materials that have been used (including the possible additional layers); (ii) the fabrication techniques employed to make the apertures; (iii) the characteristics of the resulting devices; (iv) and additional information about the BLM experimentation such as the technique chosen to prepare the BLMs, the determined seal resistance and the different types of experiments carried out on the BLMs.

\section{Table 3.1. Miniaturized devices.}

Overview of devices employing miniaturized apertures, classified according to their substrate material into siliconbased, polymer-based, glass-based and porous material-based devices. For each device, the material of the aperturecontaining substrate, the coating material, the aperture formation technique, the aperture size, the reservoir material and its size, the membrane formation methods, the position of the membrane, the seal resistance and the $B L M$ assays are mentioned. 


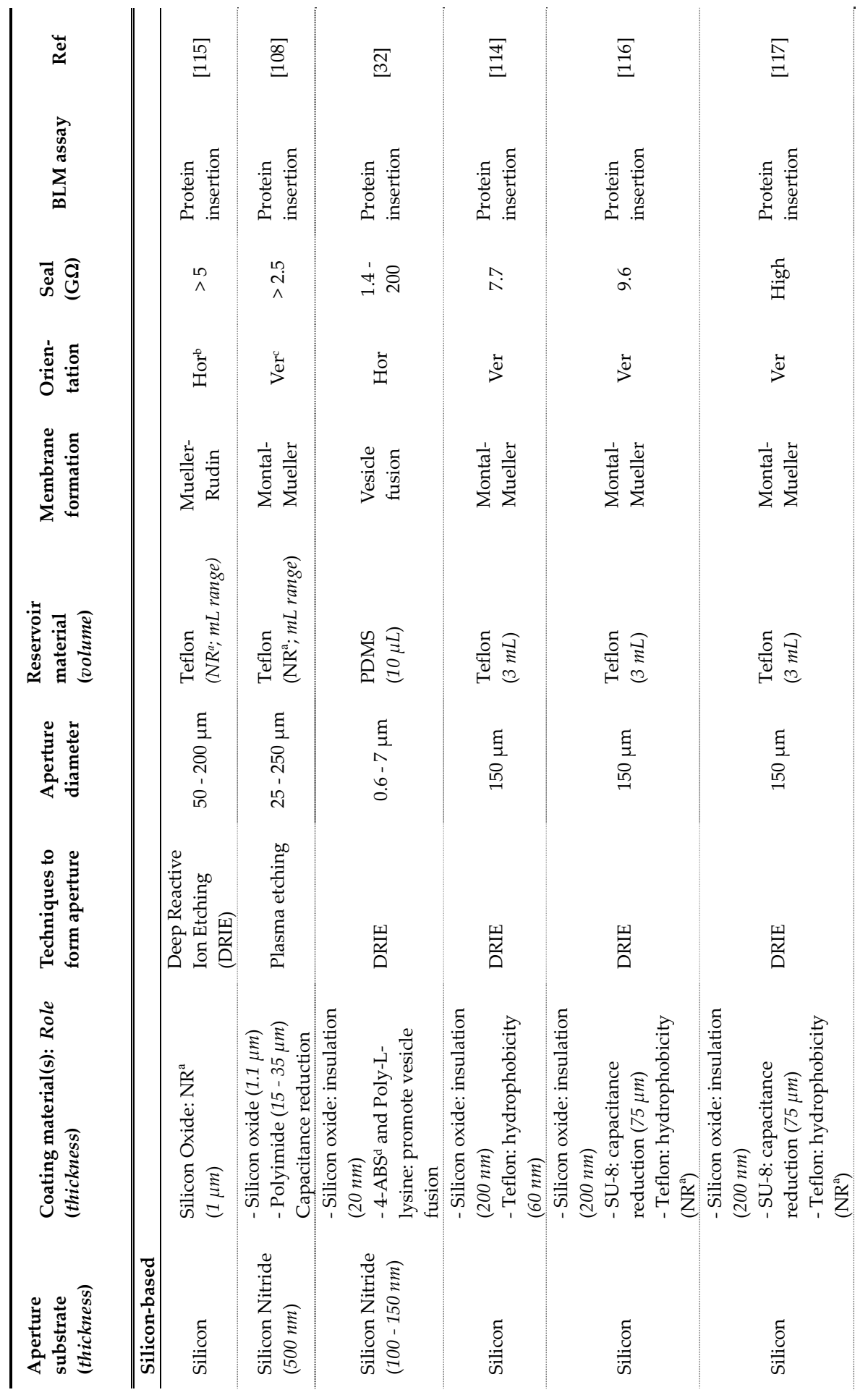




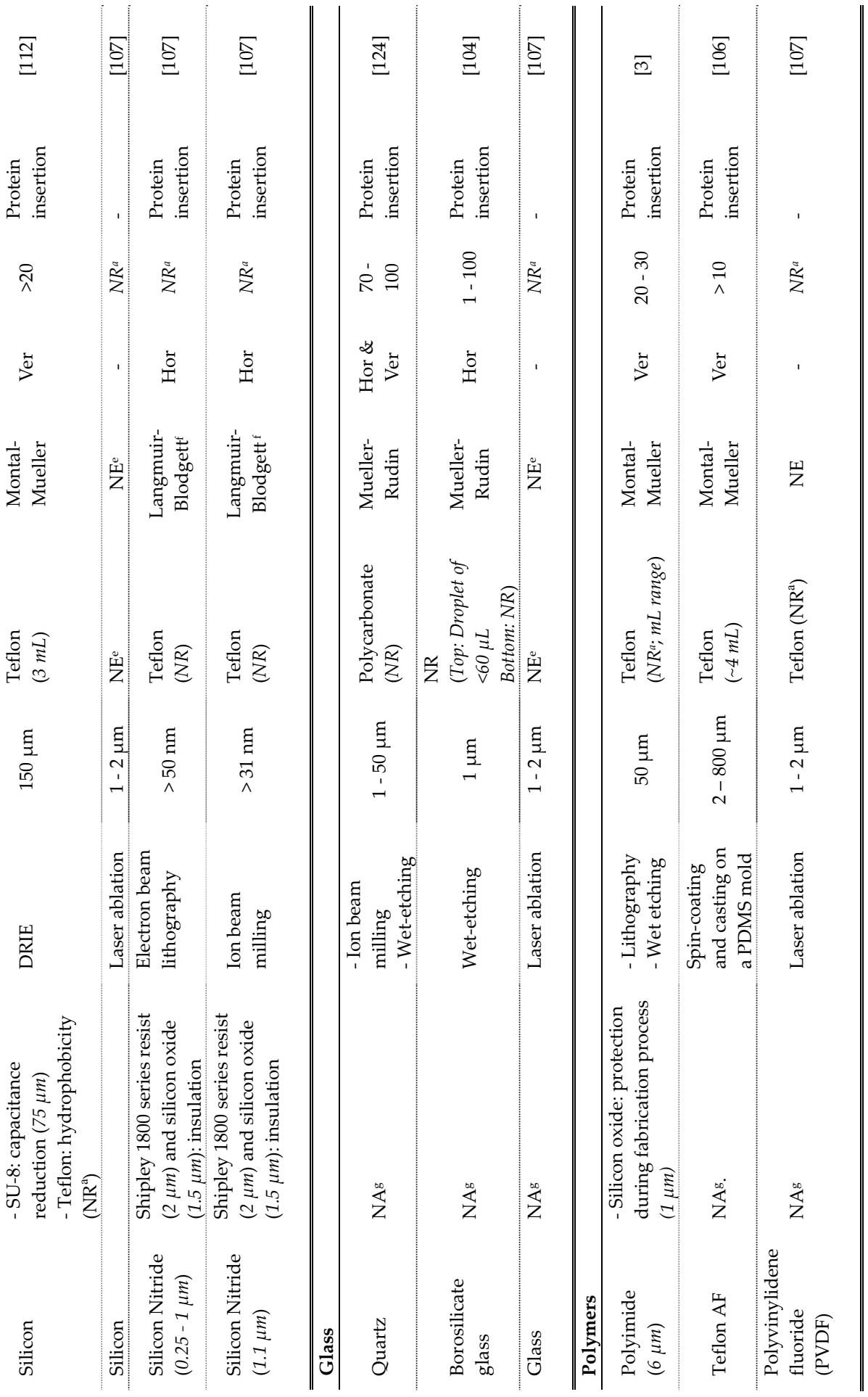




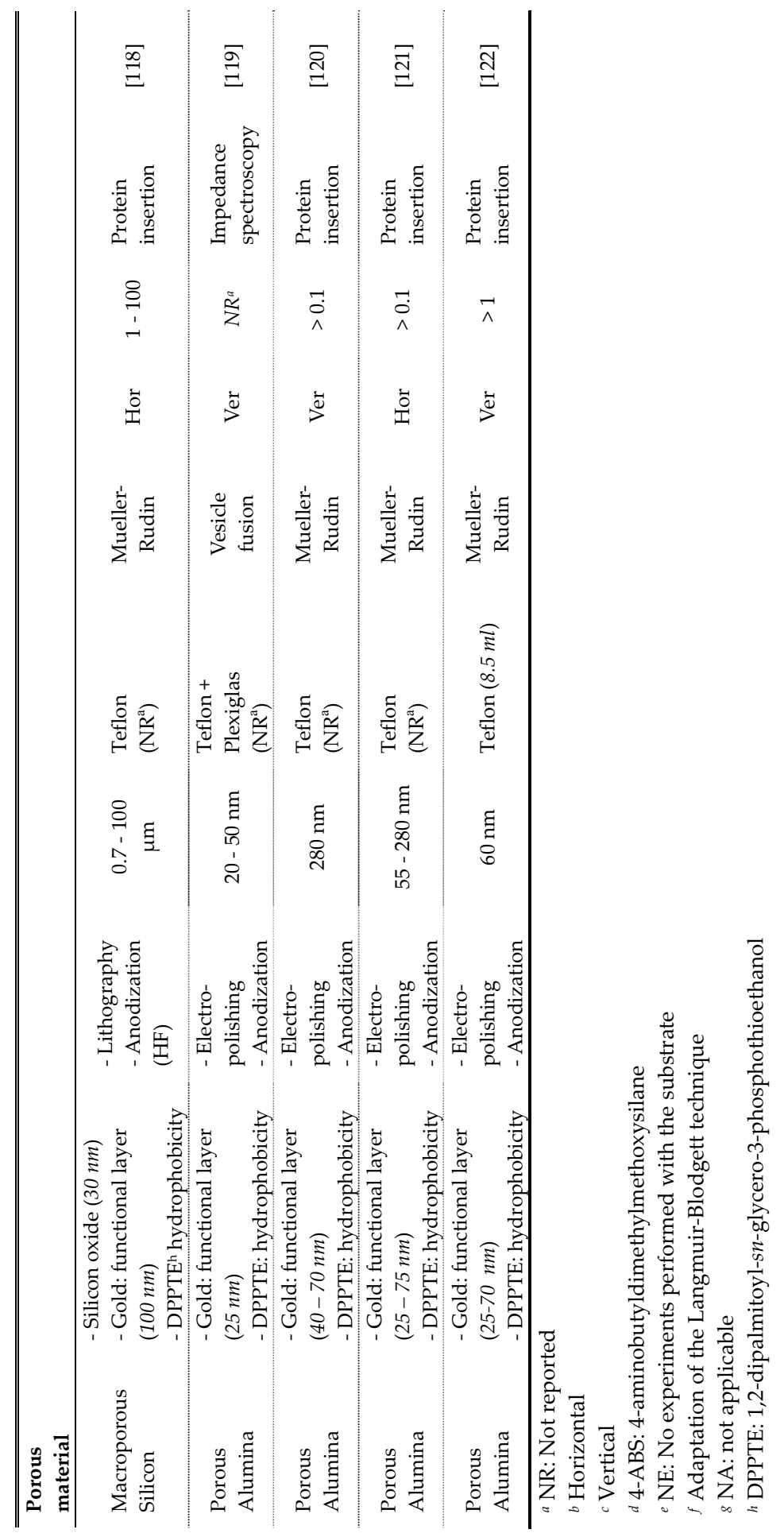


The size of the microapertures is strongly dependent on the material in which the aperture is machined and on the fabrication technique. Nanometer-sized apertures are reported for silicon, silicon nitride and porous alumina substrates [107, 113, 118-122], whereas in glass and polymer-based devices the apertures mainly remain in the micrometer range. Similarly, the seal resistance is affected by the nature of the material, and does not only depend on the size of the aperture. For instance, although the smallest apertures are reported for porous alumina substrates, the measured seal resistances are the lowest $(0.1-1$ $\mathrm{G} \Omega$ ). Conversely, the highest seal resistance is found for silicon substrates, for aperture diameters in the low micrometer range with a maximum value of $200 \mathrm{G} \Omega$ [32]. Therefore, the size of the aperture is not the only critical factor for improving BLM experimentation and other parameters such as the surface properties must be taken into account. For instance, the smoothness of the surface affects the seal quality (e.g., the rougher the surface, the less good the seal), and this depends on the fabrication process.

In general, the miniaturization of the aperture does not change the protocols for BLM experimentation. Membranes are prepared with the same standard techniques developed for conventional BLM set-ups, and any technique is in principle applicable for smaller apertures. Similarly, the same approaches as for conventional set-ups are employed to electrically characterize the BLMs. Membrane formation is monitored electrically and often confirmed by the insertion of various peptides and proteins (see table 3.1) whose activity is recorded electrically [3, 31-32, 104-106, 112, 114-118, 121]. In some devices, protein insertion is followed by studies that are more complex. Examples of those are the effects of the binding of chemicals to proteins, such as $\beta$-cyclodextrins, DNA or ATP (Adenosine triphosphate) [85, 107-108, 122] and the recording of the photocurrent generated by bacteriorhodopsin upon illumination [120]. Besides, in some of the systems the membranes are now positioned horizontally, allowing for the optical detection of the membrane surface area [115]. However, this optical information is not employed (next to the proof-of-principle aspect) to gain additional experimental freedom, since the recording of both the membrane properties and ion channel activities remains purely electrical.

The second aspect of the miniaturization of the BLM set-up concerns the fluidic reservoirs placed on both sides of the substrate containing the small aperture.

A first report found in this category aims at high-resolution and high-sensitivity optical measurements to resolve the unitary permeability of single protein transporters. The device consists of a thin transparent polycarbonate foil (100- $\mu \mathrm{m}$ thickness) containing a dense array of microapertures produced by laser drilling $(0.8-50 \mu \mathrm{m})$ and connected to microholes (30 $70 \mu \mathrm{m}$ ) or microcavities (50 fL) (See fig 3.13A). BLMs are formed in the foil using an adaptation of the Muller-Rudin technique. Following this, $\alpha$-hemolysin proteins are added in the buffer solution and self-insert in the resulting BLMs. In a first stage, BLM formation and the insertion of individual $\alpha$-hemolysin proteins in the BLMs is monitored electrically using devices with microholes, while microcavities are employed to characterize the flux of 
ions $\left(\mathrm{Ca}^{2+}\right)$ or small molecules (Calcein) through unitary pores of $\alpha$-hemolysin using confocal microscopy. In the latter case, the progressive increase in fluorescence measured in the microcavities located below the bilayers enables to quantify the flux of small molecules through $\alpha$-hemolysin proteins into this confined environment, and subsequently to determine its unitary permeability to specific small species (Calcein and $\mathrm{Ca}^{2+}$ ions, here).

A
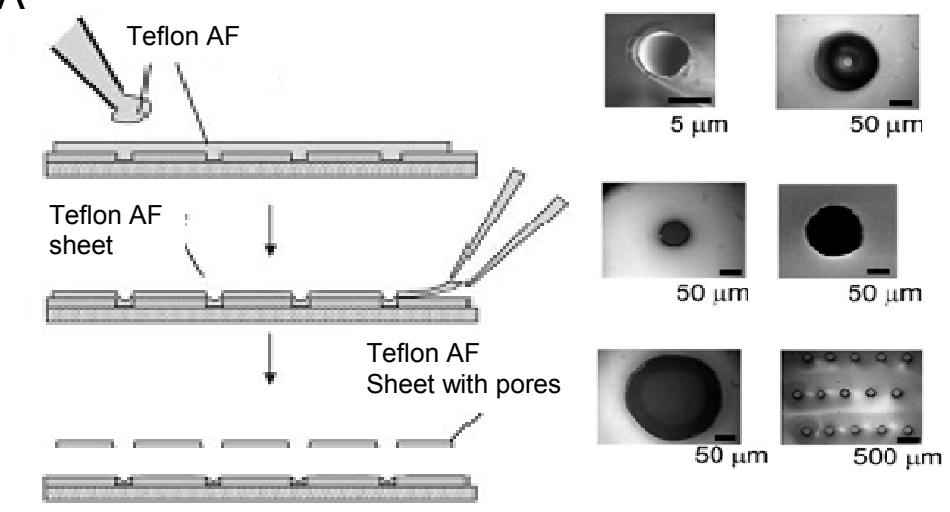

B
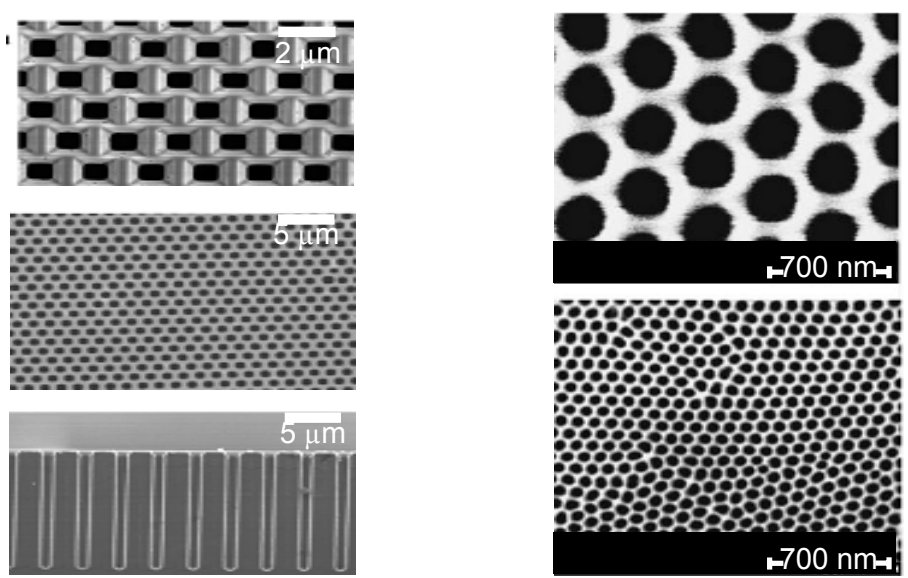

Figure 3.12. Aperture arrays.

Aperture arrays based on Teflon $(A)$ and porous materials (B). A) Left: Fabrication process of pore arrays in Teflon AF films by spin-coating it on top of a PDMS master. Right: Pictures of the pores in the Teflon films [106]. B) Left, and from top to bottom: Scanning electron microscopy (SEM) pictures of the front, bottom and side of macroporous silicon substrates covered with a gold layer (pore diameter: $1 \mu \mathrm{m}$ ) [118]. Right: SEM pictures of nanopores in porous aluminium, anodized under different conditions of 5 wt \% phosphoric acid at $2^{\circ} \mathrm{C}$ under 160 $V$ (top) and in $0.3 \mathrm{M}$ oxalic acid at $2^{\circ} \mathrm{C}$ under $40 \mathrm{~V}$ (bottom) [121]. Pore diameters are $280 \mathrm{~nm}$ (top) and $55 \mathrm{~nm}$ (bottom).

A similar approach is taken by Baaken et al. [94] but here with the goal of reducing the electrical noise, and enhance the electrical sensitivity for the BLM experimentation. Sub- 
picoliter cavities are placed under the BLMs (see figure 3.13B) to give a high-density of BLM experimentation sites on a single device. The cavities ( 3 - $10 \mu \mathrm{m}$ diam.) are produced in a 1$10 \mu \mathrm{m}$ thick SU-8 layer on a glass substrate using standard photolithography. Gold conductor lines are fabricated on the glass substrate using electron beam evaporation, before the SU-8 is spun on the glass wafer. A silver layer that forms the electrode is electrochemically deposited in each well on top of the end-points of the gold lines, after fabrication of the SU-8 microwells, and subsequently chloridized. This procedure enables to produce 16 individually addressable cavities on a single device. BLMs are formed using the Mueller-Rudin technique by swiping a Teflon stick on the SU-8 structure. Ion channel recordings (alamethicin channels and blocking of $\alpha$-hemolysin pores with PEG (Polyethylene glycol)) are performed to confirm the formation of bilayer structures and to assess the electrical performance of the device. The electrical noise is extremely low, in the fA range (against a few $\mathrm{pA}$ in standard conditions). Furthermore, simultaneous measurements on different cavities are demonstrated, showing the capability of the device for parallelization of the experimentation.

Although the miniaturization of the fluidic reservoir is less considered, this approach gives more sensitive measurements for the two most commonly used detection techniques employed to characterize BLMs, optical measurements and electrical measurements. In the former case, the confinement brought by fL-reservoirs opens the way to optical studies on a low amount of fluorescent molecules, while in the latter case, the smaller volumes considered bring about lower noise in the electrical measurements and facilitate studies on single membrane proteins.

A

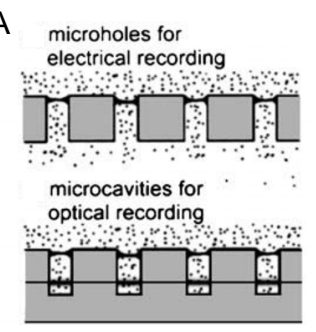

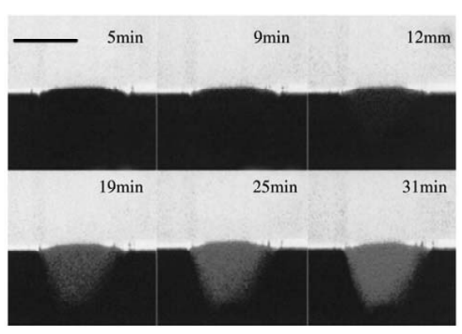

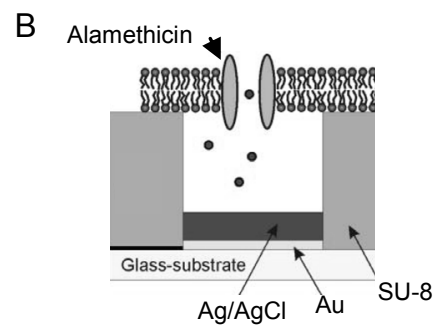

Figure 3.13. Miniaturization of the fluidic reservoirs.

A) Array of low micrometer-sized apertures employed for resolving the permeability of single $\alpha$-hemolysin protein channel; these apertures are connected to microholes for electrical measurements or microcavities for highresolution optical studies (left). Right: confocal microscopy pictures of one microcavity showing the permeability of one $\alpha$-hemolysin channel to Calcein. B) Microcavity (3-10 $\mu \mathrm{m}$ diam.) made in a $1-10 \mu \mathrm{m}$ thick SU-8 layer on top of a glass substrate. The layer of $\mathrm{Ag} / \mathrm{AgCl}$ is used as an electrode to measure protein activities [94].

\section{(ii) Half microfluidic systems}

The next class of miniaturized devices for membrane preparation includes halfmicrofluidic devices. In these systems, a microfluidic channel located below the substrate 
containing the aperture replaces one of the reservoirs while the other reservoir remains a $\mathrm{mL}-$ well. In these systems, membranes are prepared horizontally.

Two main advantages are found in half-microfluidic devices. Firstly, the space below the substrate containing the aperture is reduced, which makes optical measurements on the BLMs possible. The height of the microchannels and the thickness of the bottom substrates still dictate the quality of the optical measurements. Secondly, the presence of a microchannel provides the opportunity of flushing liquids in one reservoir and subsequently performing dynamic assays.

Interestingly, the work reported along this trend mainly originates from two research groups, while a few isolated reports are found on half-microfluidic devices.

The first two reports published along this line introduce the concept of a micromachined support for bilayers (MSB) [125-126]. A first motivation for this work is the creation of artificial cells by benefiting from the small reservoirs $(\sim 25 \mathrm{~nL})$ located below the membrane (see figure 3.14). A second driving force is the ability to study membrane transport phenomena and perform conductance measurements at the single ion channel level, towards the development of new types of biosensors. For that purpose, MSB devices bring enhanced electrical detection capabilities. A MSB device consists of a SU-8 structure that contains both a microfluidic channel $(100 \mu \mathrm{m} \times 50 \mu \mathrm{m})$ [125] and the microaperture (100 $\mu \mathrm{m}$ diam.) required for BLM experimentation, which are fabricated using standard photolithography techniques. This SU-8 structure is bonded using PDMS or an epoxy glue on a thin glass substrate, and subsequently coated with gold on which a self-assembled monolayer of a perfluoroalkanethiol is prepared to make the surface highly hydrophobic. The resulting device is mounted vertically in a PTFE (polytetrafluoroethylene) flow cell, and electrical measurements are performed using one integrated $\mathrm{Ag} / \mathrm{AgCl}$ electrode (fabricated on the thin glass substrate at the bottom of the microfluidic channel) and one $\mathrm{Ag} / \mathrm{AgCl}$ counter electrode introduced in the bulk liquid [125]. Membranes are prepared using the Montal-Mueller [126] and Mueller-Rudin [125-126] techniques, and the lifetime of the BLMs is circa 20 minutes [126]. The formation of a bilayer is verified electrically with the insertion of the single ion channels gramicidin $\mathrm{D}$ and alamethicin, or optically by adding fluorescent phospholipids in the membrane [125]. A targeted application for these MSB devices is the realization of high-sensitivity biosensors based on ligand-gated ion channel receptors.

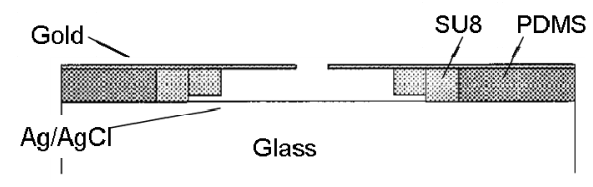

Figure 3.14. MSB device.

SU-8 channel structure containing a microfluidic channel $(100 \mu \mathrm{m} \times 50 \mu \mathrm{m})$ and an aperture (100 $\mu \mathrm{m}$ diameter) in the top layer, bonded to a glass wafer using PDMS [125-126]. 
The first group highly active in the field of miniaturization of BLM experimentation is the group of Shoji Takeuchi at the University of Tokyo (Japan). Their ultimate goal is to establish a novel platform for electrophysiology studies on single membrane proteins, called the "membrane protein chip". Interestingly, their work in this line of research starts with a fully microfluidic approach [127] but they quickly adopt half-microfluidic devices. A reason for this may be the accessibility to the BLM which is preserved in a half-microfluidic device, and that brings better control on the pressure applied on the BLM. Their work is mainly marked by the introduction of a novel methodology for BLM preparation that relies on the successive flushing of a lipid solution, air and buffer across a micrometer-sized aperture until a bilayer structure is obtained in the aperture. In that perspective, they mainly focus on the optimization of their novel BLM preparation technique, by adjusting the geometry and the features of the device. Following this, the devices are thoroughly characterized for single protein electrophysiology.

This group develops two series of microdevices for BLM experimentation. The first generation of systems is made from PMMA [27-28], and the apertures (100 $\mu \mathrm{m}$ diam.) employed as BLM experimentation sites are fabricated in a $1.5-\mathrm{mm}$ thick sheet of polymer using mechanical drilling techniques to produce a tapered structure and a microaperture at the bottom of this structure. In a second series of devices, they employ parylene as $20-\mu \mathrm{m}$ thickness membranes in which arrays of $15-50-\mu \mathrm{m}$ apertures are produced using dryetching techniques [128]. In both cases, the top reservoir consists of a large well (plastic tubing for a single BLM site against arrays of wells machined in a PMMA substrate for multiple BLM sites) and the bottom reservoirs are formed by microfluidic channels, also processed in a PMMA substrate.

In a first article, Suzuki et al. report preliminary studies on their novel methodology to prepare BLMs in a miniaturized aperture (150 $\mu \mathrm{m}$ diam.) fabricated in a silicon substrate using wet-etching techniques [127], and operated in an "open system". They deposit a small amount of a phospholipid solution $(1 \mu \mathrm{L})$ in the microaperture on a large droplet of buffer. Following this, a second droplet of buffer is added above the phospholipid solution and, when the initial layer of phospholipids is thin enough, a bilayer forms in the aperture by spontaneous thinning. BLM formation is monitored only using microscopy.

The following report includes more in-depth studies on this novel BLM preparation methodology with, more specifically, a discussion and the identification of the keyparameters that guarantee successful BLM preparation [27-28]. BLM formation is monitored using a combination of electrical and optical techniques. Two aspects are shown to be crucial: (i) the amount of lipids deposited in the aperture (thickness of the initial lipid plug); and (ii) the pressure applied to trigger thinning of the lipid plug into a bilayer by applying a hydrostatic pressure by raising the level of water in the upper well. After optimization of the whole device (height of the aperture where lipids are deposited and where BLMs are subsequently prepared) and the technique (removal of the excess of lipid solution, adjustment of the pressure applied on the lipid plug), a $90 \%$ success yield is routinely 
achieved for BLM preparation. On other aspects, the technique is demonstrated for a single BLM site and an array of four independent $100-\mu \mathrm{m}$ size apertures where four BLMs are simultaneously successfully created. Finally, the capability of the device (figure 3.15A) is shortly illustrated for single molecule electrophysiology using the pore-forming structure gramicidin, which is injected in the system from the bottom channel. Following this, the capability of the same device is fully demonstrated for electrophysiological measurements [27-28]. They firstly characterize the rms in the electrical noise in their chip $(100 \mu \mathrm{m}$ aperture); this is decreased down to $0.17 \mathrm{pA}$ in comparison with former silicon-based devices, exhibiting a noise level of up to 105 pA. Following this, ion channel species, alamethicin and a-hemolysin, are inserted in the BLM, either via liposome fusion with the membrane for the former or by the simple addition in the buffer solution, for the latter. This demonstrates the flexibility of the device for different protein integration techniques.

The following reports from this group concern a different device, where not only the aperture-containing substrate is replaced by a parylene thin sheet but where also simultaneous measurements on multiple BLM sites are described, as shown in figure 3.15B [128]. The use of a thinner substrate influences greatly the formation and the stability of the membranes; no pressure is required anymore to initiate thinning of the lipid plug and the membrane lifetime reaches $15 \mathrm{~h}$ (without insertion of any trans-membrane pores). As both the mechanical stability and seal resistance increase when the aperture size decreases and the probability of ion channel incorporation increases proportionally with the surface area of the membranes, such a layout provides the best of both worlds for protein studies. For multiplexed measurements, firstly, an array of nine independent measurement wells is fabricated in PMMA, and each well is connected to an array of 9-16 BLM apertures in the parylene sheet. The electrical activity of alamethicin and gramicidin is recorded in parallel in three separately addressed wells, which is the first report on simultaneous measurements of different trans-membrane pores inserted in independently addressable arrays of BLMs in a single device. Secondly, the multiplexed structure is extended to a 96-well array connected to eight channels both produced using the same fabrication process [111, 129]. A series of eight independent electrodes is employed for simultaneous measurements on eight BLM arrays. The procedure for BLM formation is improved further by using a mixture of two solvents, n-decane and 1-hexanol, for the phospholipid solution; this accelerates and promotes thinning of lipid plug, and enhances BLM reproducibility. Eight wells are monitored at once, after insertion of the protein $\alpha$-hemolysin in the BLMs, and the translocation events of nucleic acids are recorded simultaneously through individual nanopores. 

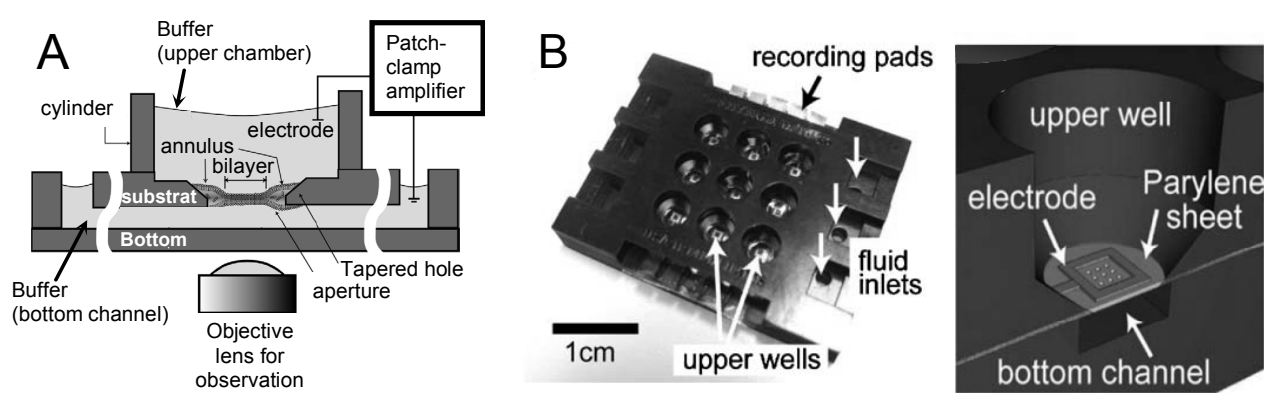

Figure 3.15. Polymer devices from the group of Shoji Takeuchi.

A) PMMA design used to optimize the membrane formation method. The substrate containing the channel $4 x$ $0.5 \mathrm{~mm}$ ), access-holes and one or four $100 \mu \mathrm{m}$ apertures is bonded to the top chamber ( $7 \mathrm{~mm}$ in diameter) and the bottom plate. The success rate for membrane formation is as 90\% [27-28]. B) Left: Photo of the device containing $4 \times 4$ individually addressable wells. Right: Schematic cross-section of one the wells containing integrated electrodes. The channels $\left(2 \times 0.5 \mathrm{~mm}^{2}\right)$ and wells $(2 \mathrm{~mm}$ diam. $)$ are formed by hybrid micro stereolithography. Half-way through the fabrication process, the 20- $\mu \mathrm{m}$ thin parylene sheet containing the apertures $(15-50 \mu \mathrm{m}$ in diameter) and integrated electrodes is placed on the device, after which fabrication is continued [111].

The second main group which have contributed noticeably to the development of miniaturized systems for BLM experimentation is the group of Hywell Morgan at the University of Southampton. As for the group of Suzuki, their first report describes the development of a microfluidic platform [123] while the rest of their work since then focuses on half-microfluidic systems. Again, this change in strategy is explained by the lack of reproducibility in membrane formation in a fully microfluidic environment, which precludes the use of such closed systems as automated and "high-throughput" platforms for membrane protein studies. A clear evolution is seen in their successive reports towards this goal of realizing "high-throughput" platforms for single protein studies. This evolution notably concerns (i) the method employed to prepare the BLM to automate this step, (ii) the design of the platform for multiplexed measurements on multiple and independent BLM sites and (iii) the demonstration of the device performance not only on model proteins but also on biologically relevant membrane proteins.

Almost all devices reported by that group are produced using the same fabrication process and materials. The polymer PMMA is mainly employed while one report mentions the use of fluoropolymers. Early reports employ silicon and glass [130-131], but this approach was quickly abandoned in favor of cheaper materials. Polymers are processed using both hot embossing to produce large structures (i.e. microchannels, wells, reservoirs) and laser-micromachining to realize the micrometer-sized apertures required for BLM experimentation in a thin substrate (50-125 $\mu \mathrm{m}$ thickness). The three substrates containing the well, the microchannels and the aperture(s), respectively, are assembled together using chemical bonding or adhesive tape, or are simply clamped. The clamping approach relies on the use of a home-made packaging device that includes additional layers based on an elastomer material to exclude any risk of leakage. 
Their first report focuses on the development of a reliable technique to create BLMs across $80-150 \mu \mathrm{m}$ diam. apertures (substrate thickness of $125 \mu \mathrm{m}$ ) in a half-microfluidic device (see figure 3.16A), and this technique should easily be amenable to automation. Membranes are formed using the "air-exposure" technique [26] that borrows much from the technique previously reported by Suzuki et al. [27]. However, here, thinning of the plug of phospholipids deposited in the aperture is triggered through its exposure to air, and not by application of a hydrostatic pressure and its exposure to a buffer solution, as detailed in section IIC of this chapter. With this technique, they report a $88 \%$ success yield for membrane formation, BLMs are stable for several hours. The performance of the device for electrical measurements is illustrated with early experiments using the pore-forming peptide, gramicidin.

In a second report, the same half-microfluidic device is applied for more biologically relevant studies on membrane proteins [48]. The potassium channel KcsA is delivered by the proteoliposome fusion technique into a membrane prepared with the air exposure technique. The vesicles containing both nystatin-ergosterol complexes and KcsA channels are fused with the BLMs by the establishment of a salt gradient across it, as discussed before [48]. With this article, Sandison et al. demonstrate the potential of their device as a single molecule biosensor.

In a next step, the platform is upgraded for multiplexed measurements. An array of 12 independent BLM sites is implemented in the thin PMMA substrate instead of a single BLM site, and the design of the rest of the platform is adapted for multiple measurements, as shown in figure 3.16B, with the use of 12 independent wells and 12 microfluidic channels [100]. Silver-based electrodes are also integrated in the platform, and they are fabricated in the bottom microfluidic layer. The air-exposure technique is still employed for membrane preparation in this system. The process of membrane thinning is studied in more detail for possible automation of its membrane formation: the time the membrane is exposed to air is critical for successful membrane formation, and this is calibrated for automation of the technique. Typically, a $50 \%$ yield is obtained in the multiplexed device for simultaneous membrane preparation against $80 \%$ if membranes are made successively while being actively monitored.

As the air exposure technique appears not to be suitable for the automated formation of membranes in an array of apertures, a second methodology of "falling droplets" [132] is developed which resembles the droplet interfacing technique [132]. The well placed above the aperture is filled with the phospholipid solution in alkane while the lower microchannel contains buffer solution. A droplet of buffer (few $\mu \mathrm{L}$ ) is dispensed in the top reservoir; it sinks to the bottom of the reservoir, down to the aperture, resulting in the spontaneous formation of a bilayer. A similar device as before is employed, where the geometry of the well has slightly been modified to a conical shape for better controlled delivery of droplets to the solvent and the aperture size is much larger $(400-800 \mu \mathrm{m}$ diam.). Membranes are formed with a $95 \%$ success yield, and exhibit an extended lifetime of 2 hours (or $<1$ hour 
after insertion of proteins). Pore-forming peptides (e.g., alamethicin or gramicidin) are inserted in the membranes; they are dissolved in a second droplet of buffer, which is successively deposited in the aperture. This technique is especially suitable for automation of the membrane formation, as no other parameter as the volume of the droplets is important to guarantee successful membrane preparation, and droplets can easily be dispensed using a robot.
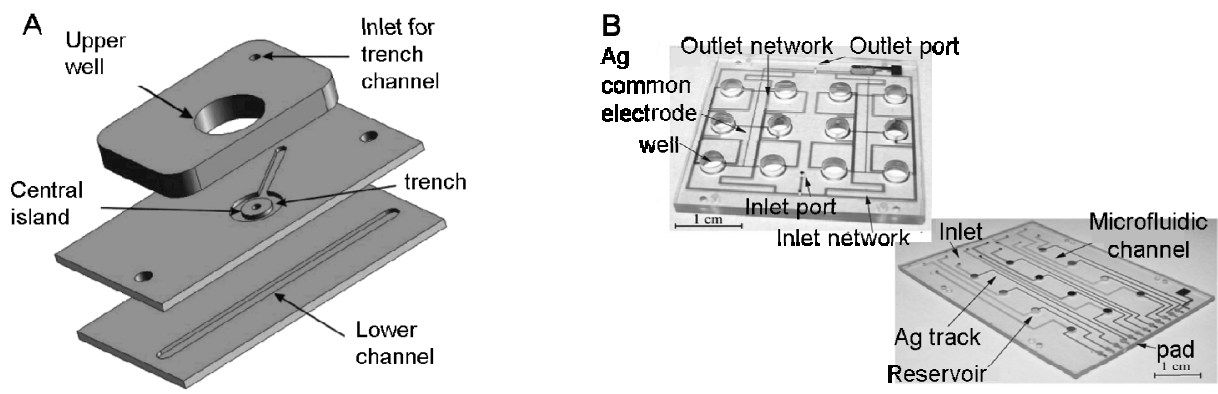

Figure 3.16. Polymer devices from the group of Hywell Morgan.

A) Fully PMMA system employed to optimize the air exposure technique. A raised area in the middle containing the $80-150 \mu \mathrm{m}$ aperture is bonded to two substrates containing a $1-2 \mathrm{~mm}$ wide channel and a well $(18 \times 18 \times 3$ $\mathrm{mm}^{3}$ ) respectively. Via the $70 \mu \mathrm{m}$ deep and $1 \mathrm{~mm}$ wide trench around this "island", solvent is added or removed from the well to form the membranes [26]. B. Photos of the top (left) and bottom (right) microfluidic parts of the multiplexed device that contains integrated $\mathrm{Ag} / \mathrm{AgCl}$ electrodes. The bottom part consists of 12 reservoirs $(2 \mathrm{~mm}$ diam.) that are individually addressable both electrically (via the integrated $\mathrm{Ag} / \mathrm{AgCl}$ electrodes) and fluidically (via $500 \mu \mathrm{m} \times 100 \mu \mathrm{m}$ channels, indicated here with food dyes). The top part also contains 12 wells that are all connected via individual channels $(500 \mu \mathrm{m} \times 100 \mu \mathrm{m})$. Consequently, 12 individually addressable BLMs are formed across 100- $\mu$ m apertures in a 50- $\mu \mathrm{m}$ thick PMMA or PTFE film [100].

Two last reports in this category could be classified either as half microfluidic devices or fully microfluidic device, as the reservoir placed above the aperture consists of a microfluidic channel that ends with a large well above the microaperture, as shown in figures 3.17A and 3.17B [133]. Thereby, chemicals can easily be flushed in the top reservoir while the aperture remains accessible for membrane formation.

The first device (figure 3.17A) is fabricated from two polycarbonate (PC) substrates using a combination of hot embossing techniques to produce the top and bottom microchannels $(60 \mu \mathrm{m} \times 50 \mu \mathrm{m})$ and mechanical drilling to form the access holes, the access well above the microaperture and the holes for the electrodes. The $20-40 \mu \mathrm{m}$ aperture is machined by burning a hole in a $12.5 \mu \mathrm{m}$ thin polyvinylidene chloride (PVDC) using a hot needle. Finally, $\mathrm{Ag} / \mathrm{AgCl}$ electrodes are fabricated on this film using thermal evaporation and electroplating. The three layers of the device are assembled and bonded together thermally in a hot press. BLMs are made using the painting method, and their formation is followed optically as well as electrically. Bilayer formation and the performance of the device are tested with the incorporation of $\alpha$-hemolysin, the electrical characterization of its 
pore and the subsequent translocation of PEG molecules through $\alpha$-hemolysin nanopores.
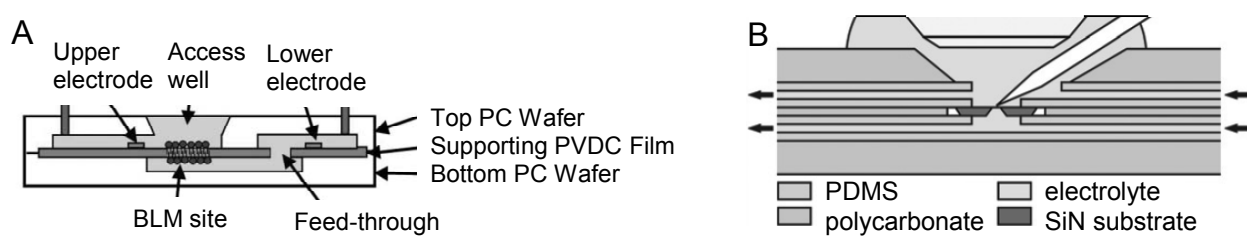

Figure 3.17. Half-microfluidic systems including an upper channel and a large well.

A) PC channel $(60 \mu \mathrm{m} \times 50 \mu \mathrm{m})$ and well structure containing a 12.5- $\mu \mathrm{m}$ thin PVDC in the middle where the aperture $(20-40 \mu \mathrm{m})$ is positioned. The channels are employed for fast buffer exchange [133]. B) Alternative PC channels and well architecture with a silicon nitride substrate containing an array of $3-12$ nanopores $(50-20$ $n m$ diam.) clamped in between. The resulting membranes have a lifetime of up to several days [134].

The second device makes use of an array of nanopores on which a large number of nanoscale-BLMs is prepared, and which is inserted between different layers of PDMS and polycarbonate (PC) to yield an integrated device (see figure 3.17B) [134]. A silicon nitride membrane is first produced on a silicon substrate by depositing silicon nitride (100-nm thickness) and by back wet-etching the silicon wafer to release a $330-\mu \mathrm{m}$ diameter freestanding membrane. Following this, the membrane area is patterned using FIB (focused ion beam) techniques to produce pore arrays or single pore of 50-600 nm diameter. The resulting substrate is finally clamped in a microfluidic cell, as shown in figure 3.17B, consisting of several layers of PDMS and PC. BLMs are created in this device by letting GUVs collapse on the nanoaperture-array, with a $90 \%$ membrane formation yield. The resulting solvent-free nanopore spanning BLMs (nsBLMs) present an extended stability and lifetime (up to several days) and are suitable for highly sensitive electrical measurements (sealing resistance of up to tens of G $\Omega$ ). The device performance is notably characterized with the insertion of poreforming peptides such as gramicidin $\mathrm{D}$ and alemithicin.

Half-microfluidic devices for BLM experimentation have emerged "late" if one considers that the first miniaturized devices appeared in the mid-90's and that the explosion of the field of microfluidics started in the late 90's. However, these devices have known a very fast development into mature platforms. The maturity of these platforms is firstly seen in the reliable, reproducible and automated process for membrane formation, most of the time using novel methodologies such as that developed by Suzuki et al. [134], the airexposure [26] and the droplet-falling [134] techniques. Membranes exhibit a longer lifetime thanks to the smaller aperture. Secondly, all platforms are suitable for highly sensitive electrical and optical measurements, on the BLMs themselves or on pore-forming peptides and even biologically relevant proteins, and are successfully applied for single molecule electrophysiology. This is obviously accounted by the miniaturization of both the aperture and the fluidic reservoirs, which dramatically decreases issues of electrical noise and 
facilitates optical characterization of the membranes. The presence of a microchannel is of great interest for rapid flushing of solutions, of proteins to be inserted in the BLM or chemicals. On other aspects, most of the proposed platforms have successfully been brought into a multiplexed format with the integration of multiple sites for BLM experimentation that are independently addressable for fluidic aspects and electrical measurements. Simultaneous measurements on unitary $\alpha$-hemolysin nanopores have been performed for the first time using a set of eight independent electrodes. Finally, all platforms are produced with a "cheap" fabrication process, employing polymer materials (PMMA, PTFE, parylene) and most of the time large-scale fabrication processes. As a conclusion, they come very close to the targeted fully automated platforms needed by the pharmaceutical industry for "highthroughput" screening of drugs on unitary protein channels.

\section{(iii) Fully microfluidic systems}

Only a few reports in the literature fall into this last category [30, 36, 123, 127, 135] in spite of the numerous additional advantages brought by fully integrated microfluidic devices. A possible reason for the limited number of examples is found in the step of membrane formation as the aperture is located in a fully closed environment while most techniques employed to prepare BLMs require the aperture to be accessible.

Most of the devices in this category consist of a sandwich composed of two substrates containing the microchannels and a thin substrate placed in between where the aperture is positioned, and the BLMs are mostly horizontal allowing for optical studies.

The first microfluidic device was developed in the group of Takeuchi and contains an array of three apertures for BLM experimentation [127]. This device is composed of a thinned oxidized silicon substrate coated with parylene for electrical insulation, and that includes $150-\mu \mathrm{m}$ apertures and the microfluidic channels $(500 \mu \mathrm{m} \times 100 \mu \mathrm{m})$ and two glass substrates on which electrodes have been patterned. The aperture, access holes and the top channel are etched into the oxidized silicon using DRIE techniques while the bottom channels are fabricated using wet-etching techniques. The three substrates are bonded together by employing SU-8 as an intermediate bonding layer [127]. BLMs are formed by filling the bottom channel with buffer and by flushing the top channel with the lipid containing solvent. The top channel is subsequently emptied to leave a thin layer of solvent in the aperture. BLM formation proceeds spontaneously when the top channel is slowly filled with buffer solution. Three BLMs are successfully created simultaneously on the three independent apertures. BLM formation is only monitored optically in spite of the presence of electrodes in the device. The buffer in the channel connects the BLMs with each other, which precludes de-coupled electrical measurements on the individual BLMs. Two parameters affect the formation of the BLMs and their reproducibility, as discussed in other articles of the same group [127]: the amount of phospholipids deposited in the aperture and the pressure applied to thin the phospholipid plug into a bilayer structure. A main 
advantage of this system is its suitability to perform lengthy optical measurements [127] as evaporation issues are alleviated in a closed system.

The second of the three-layer sandwich discussed here originates from the group of Morgan and is fully made of polymer materials [123]. Different polymers were tested (such as poly(ethylene terephthalate) (PET), poly(tetrafluoroethylene) (PTFE), and fluorinated ethylene propylene copolymer (FEP)), but poly(methyl methacrylate) (PMMA) was found to be the best material for manufacturing the device. PMMA is easy to process, transparent and its chemical resistance is satisfactory for the current application. The channel structures (500 $\mu \mathrm{m}$ wide) are formed into the PMMA by hot embossing and the $20-\mu \mathrm{m}$ apertures by lasermicromachining in a 50- $\mu \mathrm{m}$ thick PMMA film (see figure 3.18A) [123]. The three layers are bonded using solvent or thermal bonding. The device performance is demonstrated with the formation of BLMs using an adapted version of the air-exposure technique and, following this, by the insertion of gramicidin channels in the BLMs. As the materials are transparent, the BLMs are observed with microscopy techniques. However, no further information such as the membrane surface area is derived from these optical observations. As for the device discussed before, the stability and reproducibility of the membranes are not discussed. However, in a later work, the authors do mention that the membrane formation process is not very reproducible in such closed devices [26].

A further option to fabricate a multilayer system for BLM formation employs a commercially available glass substrate that contains a 1- $\mu \mathrm{m}$ size aperture [30]. In this device, the glass substrate is clamped between two PDMS layers including microfluidic channels (see figure 3.18B). The channels ( $200 \mu \mathrm{m}$ deep, $800 \mu \mathrm{m}$ wide and $15 \mathrm{~mm}$ long) are fabricated using soft-lithography techniques, and the three layers are assembled using plasmaactivation techniques. Once the device is assembled, BLMs are prepared by collapsing vesicles on the top of the aperture with the help of a negative pressure. One parameter is highly crucial to the reproducibility of the membranes: the speed of the vesicle solution in the channel must be regulated precisely for reproducible measurements. The resulting membranes are reported to exhibit a greater stability compared to membranes made with conventional techniques, although their precise lifetime is not mentioned. Following this, the noise level in the device is measured to be equivalent to what is routinely obtained with a conventional set-up, and the protein channel $\alpha$-hemolysin is inserted in the BLM.

A different approach to make BLMs in fully microfluidic devices is described by Malmstadt et al. and Funakoshi et al. [36, 135], although the device reported by Funakoshi et al. present millimeter-scale dimensions. It borrows much from the droplet interfacing technique in that the bilayer is formed between two plugs of aqueous solution through the interfacing of two monolayers of phospholipids formed at the interface between an aqueous and an organic solution. In both cases, BLM formation proceeds via the removal of the organic solvent in which the phospholipids are dissolved. However, the two groups employ 
different methods to remove the solvent.
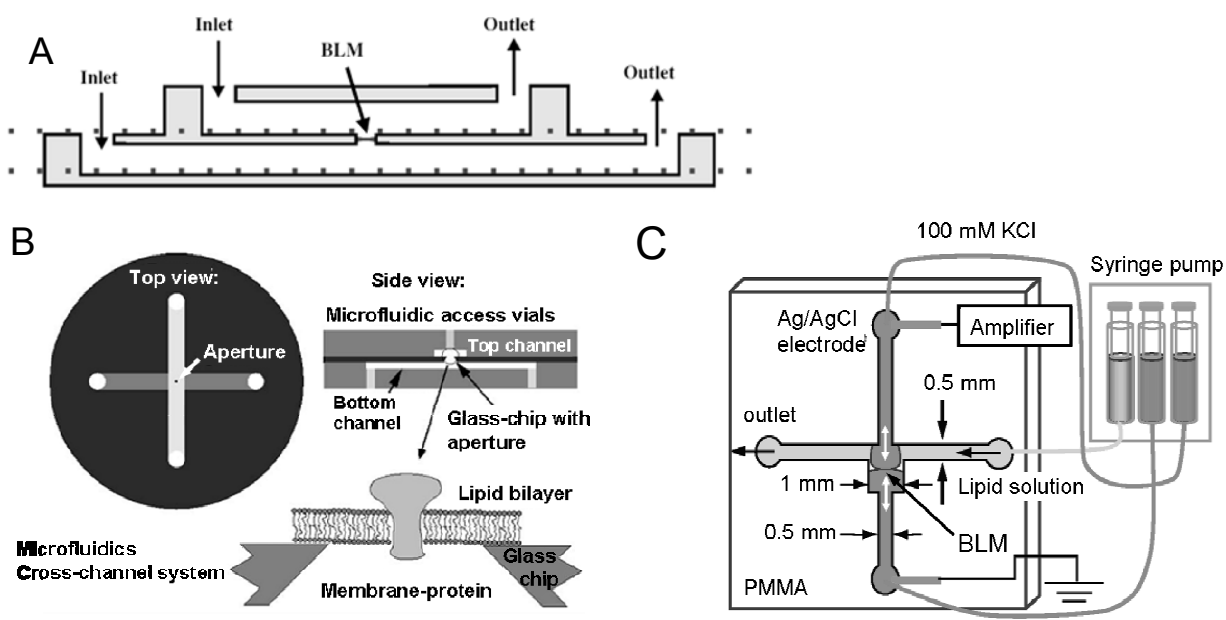

Channel depth: $0.5 \mathrm{~mm}$

Figure 3.18. Fully integrated microfluidic devices.

A) PMMA sandwich device consisting of two PMMA slabs containing the 500- $\mu m$ wide microfluidic channels with a 50- $\mu m$ thick PMMA sheet in between where the 20- $\mu m$ aperture is fabricated in [123]. B) Alternative sandwich device made using a commercially available glass slide containing the aperture [30]. Left: Top view of the two crossing channels (200 $\mu \mathrm{m}$ deep and $800 \mu \mathrm{m}$ wide) in PDMS with the 1- $\mu \mathrm{m}$ aperture in the middle. Right, top: Side view of the stacked device; the glass slide is clamped between the two PDMS slabs. Right, bottom: Zoom of the membrane containing a protein sitting on top of the aperture. C) Schematic drawing of a chip where a vertical BLM is formed at the channel intersection. When the aqueous solution comes in contact with the phospholipid-containing organic solution, a monolayer of lipids forms at the interface. When the two aqueous solutions are brought into contact, a bilayer forms [36].

Malmstadt et al. use a PDMS-based device and exploit the fact that organic solvents diffuse into the PDMS. A small plug of organic solvent containing a low concentration of lipids is introduced between two aqueous plugs in a PDMS microfluidic channel $(100 \mu \mathrm{m}$ wide). As the solvent (1:1 n-decane-squalene) is extracted through the PDMS, the two phospholipid monolayers present at the water/organic interface eventually meet to give a bilayer. The formation of the bilayer is confirmed by the insertion of the channel-forming protein $\alpha$-hemolysin, which is monitored electrically with the help of two electrodes inserted in the system.

The device of Funakoshi et al. is made from PMMA and consists of two crossing channels $(0.5 \mathrm{~mm} \times 0.5 \mathrm{~mm})$. One of the channels presents a wider section close to the channel intersection and is employed to remove the solvent between the phospholipid monolayers (see figure 3.18C) [36]. BLMs are formed as follows. The channel with a constant cross-section is firstly filled with the phospholipid solution in n-decane. In the other channel, an aqueous buffer is pumped from both sides until the two aqueous plugs meet in the wider section of the channel. The organic solution is expulsed between the two aqueous 
flows to give a bilayer, BLM formation is confirmed with the insertion of gramicidin A and its dimerization to form channels in the membrane. With this methodology, membranes can be formed repeatedly, broken and formed again.

Although these two systems appear to be attractive as BLMs form easily, their utilization remains limited in terms of applications and they do not present most of the advantages sought in microfluidic devices such as potential optical detection, fast perfusion of liquid on both sides of the membrane, and small-sized reservoirs.

The use of these microfluidic devices for experimentation on membrane models is still in its infancy. A low number of devices truly fit into this last category of fully-microfluidic systems and most of the experimentation reported is limited to proof-of-principle experiments (BLM formation, insertion of pore-forming species). One possible reason for this is the limited accessibility to the aperture, which appears to be an issue for membrane formation. In both miniaturized and half-microfluidics devices, existing preparation techniques are easily employed, and simply downscaled. On the contrary, in microfluidic devices, the aperture across which the BLMs are prepared is not accessible so that other techniques must be developed to produce BLMs. A key-issue in a microfluidic context is to precisely control the amount of phospholipid solution injected into the device to form the bilayer, which is cumbersome as pL-volumes are considered. Several methodologies rely on thinning of a phospholipid plug into a bilayer [26-28, 127-129]. There, the thickness of the lipid plug is decisive for its spontaneous thinning. A thin plug spontaneously thins into a bilayer after application of a pressure (air, buffer flow) while a too thick plug is likely not to evolve [127]. The pressure applies is also very critical in this process; a too high pressure breaks the lipid plug while a too low pressure does not affect the lipid plug.

\section{Microfluidics: A future standard for BLM experimentation?}

Cell membranes are very complex structures, and simplified models are required to understand precise molecular interactions taking place in the membrane and the specific functioning of its constituents. The simplest and most flexible models available are planar bilayer lipid membranes, or BLMs. As suggested by their name, these structures are planar patches of a phospholipid bilayer, and they are suspended across an aperture or supported on top of a surface. The complexity of these models can range from one phospholipid component to a mixture of different phospholipid components, or a mixture of phospholipids and cholesterol and/or proteins, depending on the targeted application.

Conventional systems to form suspended BLMs consist of two large reservoirs separated by a $150 \mu \mathrm{m}-1 \mathrm{~mm}$ diameter aperture in a certain substrate. For these devices, different membrane formation techniques have been developed, each presenting specific advantages and drawbacks. For instance, the Mueller-Rudin technique is probably the simplest method to form a membrane, but due to the ever-present layer of solvent between the two bilayer leaflets (unless specific solvents such as squalene are employed), and their relatively large annulus, the membrane is poorly reproducible in terms of thickness and 
surface area. On other aspects, techniques based on the Langmuir-Blodgett approach, such as the Montal-Mueller technique, are more cumbersome, and not suited for every phospholipid, but they are more reproducible in terms of the properties of the BLMs.

BLMs are of great interest for different fields of investigations, ranging from fundamental studies on the membrane properties to their use as matrixes for membrane proteins. The latter scenario requires protein to be inserted into the membranes, and again different strategies are conceivable depending on the protein of interest. However, two main approaches dominate; soluble proteins are added to the buffer solution and insert spontaneously while most of the proteins that must remain in a membrane-like environment, are isolated in a vesicle, which is fused with the BLM.

Conventional devices present a number of drawbacks. Firstly, BLMs are formed vertically, limiting their characterization to electrical techniques and precluding optical measurements, which would bring along additional information. Secondly, the large reservoirs and aperture reduce the electrical sensitivity of the systems. A high electrical sensitivity is especially crucial when working with single protein channels. The large volume of the reservoirs also results in longer diffusion times for molecules added in solution. Consequently, not only the experimentation time is increased, but also the probability for an unsuccessful outcome becomes larger, as the risk for membrane rupture rises over time.

A possible route to alleviate these issues consists of using miniaturized and/or microfluidic devices for BLM experimentation. In most of the cases, membranes are formed horizontally, extending the range of characterization schemes to optical and scanning techniques. Smaller apertures bring along more stable membranes, and the opportunity for longer experimentations. This finds a particular interest in screening assays, where (many) compounds are flushed successively on the same membrane. The electrical noise level also decreases dramatically for smaller membranes as both the sealing resistance and the capacitance of the membrane becomes higher. A last, and maybe the most promising aspect found in miniaturized devices is the possibility to realize automated and multiplexed systems, containing arrays of individually addressable apertures (both fluidically and electrically), which would enable "high-throughput" studies on the properties of membranes and membrane proteins.

Miniaturized devices can be classified into three categories presenting more and more microfluidics: miniaturized, half-microfluidics and fully-microfluidics. The two latter appear as good candidates to become novel golden standard in the field of BLM experimentation. They present additional values compared to the miniaturized devices; they include smaller reservoirs, liquid exchange is faster when a microfluidic channel is implemented, and they lend themselves better to the realization of multiplexed and automated systems for membrane-based studies.

Two fully-microfluidic systems are already commercially available in that line of 
miniaturized devices, the port-o-patch system from Nanion Technologies $\mathrm{GmbH}$ (München, Germany) and the bilayer explorer from Ionovation $\mathrm{GmbH}$ (Osnabrück, Germany), and both systems are designed for membrane protein studies [48-49, 138-140]. However, for both sensing and pharmaceutical applications, multiplexed systems are highly desired, to test simultaneously a large number of parameters under similar experimentation conditions, whereas these two systems include a single BLM experimentation site. On other aspects, they do not fully belong to the category of microfluidic devices although the fluidic reservoirs are smaller to decrease the perfusion times.

However, the question remains; which level of miniaturization is going to become predominant? This is likely to be influenced by the application the system is designed for. The key-difference between both approaches is the accessibility of the BLMs, and the question is whether this is crucial or not for automated experimentation, and notably for the preparation of BLMs. The system developed by Ionovation GmbH or the work of Mach et al. [30] demonstrate that membrane formation can be automated in a closed device, while being reproducible. Real required key-features of such a BLM platform are the ease of membrane formation, whatever technique is employed for this step, enhanced detection capability (decrease in electrical noise and coupling to optical techniques), no issue of solvent evaporation and possible fast perfusion on both sides of the membranes, and these are found in both half-microfluidic and fully microfluidic devices.

To conclude, miniaturization and microfluidics both provide a large number of advantages compared to conventional set-ups as well as opportunities for the automation and multiplexing of BLM assays. It is not to be doubted that the coming years will see a fast development in these devices, especially for "high-throughput" screening assays of drugs on membrane proteins or the development of novel types of biosensors.

\section{References}

[1] Mueller, P. et al. (1963). J. Phys. Chem 67 (2), pp. 534-535.

[2] Tamm, L.K. \& Mcconnell, H.M. (1985). Biophys J 47 (1), pp. 105-113.

[3] Eray, M. et al. (1994). Biosens. Bioelectron. 9 (4-5), pp. 343-351.

[4] Castellana, E.T. \& Cremer, P.S. (2006). Surf Sci Rep 61 (10), pp. 429-444.

[5] Gorter, E. \& Grendel, F. (1925). J. Exp. Med. 41 (4), pp. 439-443.

[6] Tien, H.T. \& Ottova, A.L. (2001). J Membrane Sci 189 (1), pp. 83-117.

[7] Heimburg, T. (2007). Thermal Biophysics of Membranes. Wiley-VCH Verlag GmbH: Wienheim, Germany

[8] Huang, J.Y. \& Feigenson, G.W. (1999). Biophys J 76 (1), pp. A272-A272.

[9] Ohvo-Rekila, H. et al. (2002). Prog Lipid Res 41 (1), pp. 66-97.

[10] Alberts, B. et al. (1989). Molecular Biology of the Cell. Garland Publishing, Inc: New York, NY, USA

[11] Cornell, B.A. \& Separovic, F. (1983). BBA 733 (1), pp. 189-193.

[12] Koronkiewicz, S. \& Kalinowski, S. (2004). BBA-Biomembranes 1661 (2), pp. 196-203.

[13] Lundbaek, J.A. et al. (2010). J R Soc Interface 7 (44), pp. 373-395. 
[14] van Uitert, I. et al. (2010). BBA - Biomembranes 1798 (1), pp. 21-31.

[15] Miedema, H. et al. (2004). Biophys J 87 (5), pp. 3137-3147.

[16] Nikolelis, D.P. et al. (1996). Bilayer lipid membranes and other lipd-based methods. In Handbook of Chemical and Biological Sensors, pp 221-256.

[17] Schindler, H. \& Quast, U. (1980). Proceedings of the National Academy of Sciences of the United States of America-Biological Sciences 77 (5), pp. 3052-3056.

[18] Matsuno, Y. et al. (2004). Analytical Sciences 20 (8), pp. 1217-1221.

[19] White, S.H. (1978). Biophys J 23 (3), pp. 337-347.

[20] Chernomordik, L.V. et al. (1985). Biochim Biophys Acta 812 (3), pp. 643-655.

[21] Montal, M. \& Mueller, P. (1972). P Natl Acad Sci USA 69 (12), pp. 3561-3566.

[22] Kalb, E. et al. (1992). Biochim Biophys Acta 1103 (2), pp. 307-316.

[23] Wiese, A. \& Seydel, U. (2000). Electrophysiological measurements on reconstituted outer membranes. In Methods in molecular biology., Vol. 145.

[24] Krylov, A.V. et al. (2001). J Gen Physiol 118 (4), pp. 333-339.

[25] Coronado, R. \& Latorre, R. (1983). Biophys J 43 (2), pp. 231-236.

[26] Sandison, M.E. et al. (2007). Langmuir 23 (15), pp. 8277-8284.

[27] Suzuki, H. et al. (2006). Langmuir 22 (4), pp. 1937-1942.

[28] Suzuki, H. et al. (2007). Biosens Bioelectron 22 (6), pp. 1111-1115.

[29] Tao, T. et al. (1996). Biophys J 70 (2), pp. 743-753.

[30] Mach, T. et al. (2008). Analytical and Bioanalytical Chemistry 390 (3), pp. 841-846.

[31] Sondermann, M. et al. (2006). Bba-Biomembranes 1758 (4), pp. 545-551.

[32] Schmidt, C. et al. (2000). Angew Chem Int Edit 39 (17), pp. 3137-3140.

[33] Dudia, A. et al. (2008). Nano Lett 8 (4), pp. 1105-1110.

[34] Aghdaei, S. et al. (2008). Lab Chip 8 (10), pp. 1617-1620.

[35] Bayley, H. et al. (2008). Mol Biosyst 4 (12), pp. 1191-1208.

[36] Funakoshi, K. et al. (2006). Analytical Chemistry 78 (24), pp. 8169-8174.

[37] Garstecki, P. et al. (2006). Lab Chip 6 (5), pp. 693-693.

[38] Link, D.R. et al. (2004). Phys Rev Lett 92 (5).

[39] Shui, L. et al. (2007). Adv. Colloid Interface Sci. 133 (1), pp. 35-49.

[40] Holden, M.A. et al. (2007). J Am Chem Soc 129 (27), pp. 8650-8655.

[41] Nozawa, K. et al. (2007). Sensor Actuat B-Chem 126 (2), pp. 632-640.

[42] White, R.J. et al. (2007). J Am Chem Soc 129 (38), pp. 11766-11775.

[43] Miller, C. \& Racker, E. (1976). J Membr Biol 30 (3), pp. 283-300.

[44] Wolosin, J.M. (1980). Biochem J 189 (1), pp. 35-44.

[45] Holloway, P.W. (1973). Anal Biochem 53 (1), pp. 304-8.

[46] Woodbury, D.J. \& Rognlien, K. (2000). Cell Biol Int 24 (11), pp. 809-818.

[47] de Planque, M.R.R. et al. (2006). Iee P-Nanobiotechnol 153 (2), pp. 21-30.

[48] Zagnoni, M. et al. (2007). Lab Chip 7 (9), pp. 1176-1183.

[49] Hirano-Iwata, A. et al. (2008). Trac-Trend Anal Chem 27 (6), pp. 512-520.

[50] van Uitert, I. et al. (2010). Biochimica et Biophysica Acta (BBA) - Biomembranes 1798 (1), pp. 21-31.

[51] Hendrich, A.B. \& Michalak, K. (2003). Curr. Drug Targets 4 (1), pp. 23-30.

[52] Benatti, P. et al. (2004). J Am Coll Nutr 23 (4), pp. 281-302.

[53] Yaqoob, P. \& Shaikh, S.R. (2010). Curr Opin Clin Nutr 13 (2), pp. 156-166. 
[54] Siu, S.W.I. \& Bockmann, R.A. (2007). Journal of Structural Biology 157 (3), pp. 545-556.

[55] Troiano, G.C. et al. (1999). Biophys. J. 76 (6), pp. 3150-3157.

[56] Brown, D.A. \& London, E. (2000). J. Biol. Chem. 275 (23), pp. 17221-17224.

[57] Veatch, S.L. et al. (2004). Biophys. J. 86 (5), pp. 2910-2922.

[58] Vist, M.R. \& Davis, J.H. (1990). Biochemistry 29 (2), pp. 451-464.

[59] Feigenson, G.W. \& Buboltz, J.T. (2001). Biophys. J. 80 (6), pp. 2775-2788.

[60] Veatch, S.L. \& Keller, S.L. (2005). Phys. Rev. Lett. 94 (14).

[61] Alecio, M.R. et al. (1982). Proc Natl Acad Sci U S A 79 (17), pp. 5171-4.

[62] de Almeida, R.F.M. et al. (2003). Biophys. J. 85 (4), pp. 2406-2416.

[63] Rubenstein, J.L. et al. (1979). Proc Natl Acad Sci U S A 76 (1), pp. 15-8.

[64] van Rooijen, B.D. et al. (2009). Biochim Biophys Acta 1788 (6), pp. 1271-8.

[65] Milhiet, P.E. et al. (2001). Biophys. J. 81 (1), pp. 547-555.

[66] Milhiet, P.E. et al. (2002). J. Biol. Chem. 277 (2), pp. 875-878.

[67] Yuan, C.B. \& Johnston, L.J. (2001). Biophys. J. 81 (2), pp. 1059-1069.

[68] Quist, A.P. et al. (2007). Langmuir 23 (3), pp. 1375-1380.

[69] Sullan, R.M. et al. (2009). Langmuir 25 (13), pp. 7471-7.

[70] van Uitert, I. et al. (2010). Soft Matter 6 (18), pp. 4420-4429.

[71] Navarrete, E.G. \& Santos-Sacchi, J. (2006). Biophys J 90 (3), pp. 967-974.

[72] Marrink, S.J. et al. (2009). Bba-Biomembranes 1788 (1), pp. 149-168.

[73] Tieleman, D.P. (2004). BMC Biochem 5.

[74] Tieleman, D.P. et al. (2003). J. Am. Chem. Soc. 125 (21), pp. 6382-6383.

[75] Berkowitz, M.L. (2009). Biochim Biophys Acta 1788 (1), pp. 86-96.

[76] Ipsen, J.H. et al. (1987). Biochim Biophys Acta 905 (1), pp. 162-72.

[77] Mouritsen, O.G. (1991). Chem Phys Lipids 57 (2-3), pp. 179-94.

[78] Nielsen, M. et al. (1999). Phys Rev E Stat Phys Plasmas Fluids Relat Interdiscip Topics 59 (5 Pt B), pp. 5790-803.

[79] Risselada, H.J. \& Marrink, S.J. (2008). Proc Natl Acad Sci U S A 105 (45), pp. 17367-72.

[80] Tieleman, D.P. et al. (2003). J Am Chem Soc 125 (21), pp. 6382-3.

[81] Tien, H.T. \& Ottova-Leitmannova, A., Planar Lipid Bilayers (BLM's) and Their Applications (Membrane Science and Technology) Elsevier Science: 2003.

[82] Bashford, C.L. (2004). Eur Biophys J 33 (3), pp. 280-2.

[83] Kasianowicz, J.J. \& Bezrukov, S.M. (1995). Biophys J 69 (1), pp. 94-105.

[84] Menestrina, G. (1986). J Membrane Biol 90 (2), pp. 177-190.

[85] Ervin, E.N. et al. (2008). Anal Chem 80 (6), pp. 2069-2076.

[86] Karginov, V.A. et al. (2007). Bioorgan Med Chem 15 (16), pp. 5424-5431.

[87] Miedema, H. et al. (2006). Eur Biophys J Biophy 36 (1), pp. 13-22.

[88] Miedema, H. et al. (2007). Nano Lett 7 (9), pp. 2886-2891.

[89] Kocer, A. et al. (2005). Science 309 (5735), pp. 755-758.

[90] Nielsen, C.H. (2009). Anal Bioanal Chem 395 (3), pp. 697-718.

[91] Gouaux, E. (1998). Journal of Structural Biology 121 (2), pp. 110-122.

[92] Song, L.Z. et al. (1996). Science 274 (5294), pp. 1859-1866.

[93] Kasianowicz, J.J. et al. (1996). P Natl Acad Sci USA 93 (24), pp. 13770-13773.

[94] Baaken, G. et al. (2008). Lab on a Chip 8 (6), pp. 938-944.

[95] Meller, A. et al. (2001). Phys Rev Lett 86 (15), pp. 3435-3438. 
[96] Vercoutere, W. et al. (2001). Nat Biotechnol 19 (3), pp. 248-252.

[97] Howorka, S. et al. (2001). P Natl Acad Sci USA 98 (23), pp. 12996-13001.

[98] Brüggemann, A. et al. (2003). Assay Drug Dev. Technol. 1 (5), pp. 665-673.

[99] Brüggemann, A. et al. (2004). Curr Drug Discov Technol 1 (1), pp. 91-6.

[100] Zagnoni, M. et al. (2009). Biosens Bioelectron 24 (5), pp. 1235-1240.

[101] Whitesides, G.M. (2006). Nature 442 (7101), pp. 368-373.

[102] Becker, H. \& Gartner, C. (2008). Anal Bioanal Chem 390 (1), pp. 89-111.

[103] van den Berg, A. \& Bergveld, P. (2006). Lab Chip 6 (10), pp. 1266-73.

[104] Fertig, N. et al. (2002). Biophysical Journal 82 (6), pp. 3056-3062.

[105] Fertig, N. et al. (2001). Phys. Rev. E 6404 (4).

[106] Mayer, M. et al. (2003). Biophys J 85 (4), pp. 2684-2695.

[107] McGeoch, J.E.M. et al. (2000). Med Biol Eng Comput 38 (1), pp. 113-119.

[108] Peterman, M.C. et al. (2002). Biomed Microdevices 4 (3), pp. 231-236.

[109] Hemmler, R. et al. (2005). Biophys. J. 88 (6), pp. 4000-4007.

[110] Simon, A. et al. (2007). J. Colloid Interface Sci. 308 (2), pp. 337-343.

[111] Suzuki, H. et al. (2009). Biomed Microdevices 11 (1), pp. 17-22.

[112] Wilk, S.J. et al. (2007). Biosens. Bioelectron. 23 (2), pp. 183-190.

[113] Fertig, N. et al. (2000). Appl Phys Lett 77 (8), pp. 1218-1220.

[114] Wilk, S.J. et al. (2004). Applied Physics Letters 85 (15), pp. 3307-3309.

[115] Pantoja, R. et al. (2001). Biophysical Journal 81 (4), pp. 2389-2394.

[116] Wilk, S.J. et al. In NSTI Nanotechnology Conference 2005, 2005; Nano Science and Technology Institute, Cambridge, MA 02139, United States, Vol. 2, pp 504-507.

[117] Wilk, S.J. \& et al. (2006). J. Phys. 38 (1), p. 21.

[118] Romer, W. et al. (2004). J Am Chem Soc 126 (49), pp. 16267-16274.

[119] Drexler, J. \& Steinem, C. (2003). Journal of Physical Chemistry B 107 (40), pp. 1124511254.

[120] Horn, C. \& Steinem, C. (2005). Biophys J 89 (2), pp. 1046-1054.

[121] Romer, W. \& Steinem, C. (2004). Biophysical Journal 86 (2), pp. 955-965.

[122] Schmitt, E.K. et al. (2006). Biophys J 91 (6), pp. 2163-2171.

[123] Sandison, M.E. \& Morgan, H. (2005). J Micromech Microeng 15 (7), pp. S139-S144.

[124] Fertig, N. et al. (2001). Physical Review E 6404 (4).

[125] Cheng, Y.L. et al. (2001). Langmuir 17 (4), pp. 1240-1242.

[126] Ogier, S.D. et al. (2000). Langmuir 16 (13), pp. 5696-5701.

[127] Suzuki, H. et al. (2004). Lab Chip 4 (5), pp. 502-505.

[128] Le Pioufle, B. et al. (2008). Anal Chem 80 (1), pp. 328-332.

[129] Osaki, T. et al. (2009). Anal. Chem. 81 (24), pp. 9866-9870.

[130] Sandison, M.E. et al. (2007). J Micromech Microeng 17 (7), pp. S189-S196.

[131] Morgan, H. et al. In Proceedings of uTAS 2004 2004, Vol. 1, pp 330-332.

[132] Zagnoni, M. et al. (2009). Anal Bioanal Chem 393 (6-7), pp. 1601-1605.

[133] Hromada, L.P. et al. (2008). Lab Chip 8 (4), pp. 602-608.

[134] Kresak, S. et al. (2009). Soft Matter 5 (20), pp. 4021-4032.

[135] Malmstadt, N. et al. (2006). Nano Lett 6 (9), pp. 1961-1965. 


\section{Chapter 4}

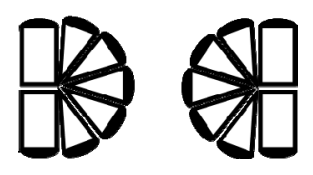

\section{The influence of different membrane components on the electrical stability of bilayer lipid}

\section{membranes}

A good understanding of cell membrane properties is crucial for better controlled and reproducible experiments, particularly for cell electroporation where the mechanism of pore formation is not fully elucidated. In this chapter we describe the influence on that process of several constituents found in natural membranes using bilayer lipid membranes. This is achieved by measuring the electroporation threshold $\left(V_{t h}\right)$ defined as the potential at which pores appear in the membrane. We start from highly stable 1,2-diphytanoyl-sn-glycero-3phosphocholine (DPhPC) membranes $\left(V_{t h} \sim 200 \mathrm{mV}\right.$ ), and subsequently add therein other phospholipids, cholesterol and a channel protein. While the phospholipid composition has a slight effect $\left(100 \mathrm{mV} \leq V_{\text {th }} \leq 290 \mathrm{mV}\right)$, cholesterol gives a concentration-dependent effect: a slight stabilization until $5 \%$ weight $\left(V_{t h} \sim 250 \mathrm{mV}\right.$ ) followed by a noticeable destabilization ( $V_{\text {th }} \sim 100 \mathrm{mV}$ at $20 \%$ ). Surprisingly, the presence of a channel protein, $\alpha$-hemolysin, does not affect the sensitivity of the membrane to the application of an electroporation voltage. A reason for this absence of effect is obviously to be found in the low protein density $\left(1.1 \times 10^{-3}\right.$ proteins $/ \mu \mathrm{m}^{2}$ ) that could be tested here. In general, we find that pore formation is affected by the molecular organization (packing and ordering) in the membrane and by its thickness. We correlate the resulting changes in molecular interactions to theories on pore formation.

Part of this chapter was published in BBA-Biomembranes, 1: 21-31 (2010). 


\section{Introduction}

As mentioned in the previous chapter, a cell membrane consists of a (phospho)lipid matrix defining its structure and shape and serving as a substrate for membrane proteins. This matrix consists of a large variety of phospholipids together with a substantial amount of cholesterol (typically 30\%). Cholesterol greatly affects the fluidity of the membrane by establishing specific interactions with the hydrocarbon chains and head groups of the phospholipids [1]. The last main component found in cell membranes is proteins which account for $50 \%$ of the membrane weight [2]. The so-called transmembrane proteins span the membrane and present hydrophilic parts eventually protruding on both sides and a hydrophobic part located in the hydrophobic core of the membrane.

This cell membrane forms an impermeable barrier to foreign entities, among which genes, drugs, particles, and certain dyes. For many applications, such as DNA transfection or drug research, it is necessary to transport these substances into a cell, and this requires the transient permeabilization of the cell membrane. A commonly used technique for this purpose is electroporation [3-5]. Thereby, pores are temporarily created in the membrane upon application of a high external electric field $(\mathrm{kV} / \mathrm{cm})$, usually short DC pulses ( $\mu \mathrm{s}-\mathrm{ms}$ range) or exponentially decaying pulses [6]. When the imposed transmembrane potential reaches a threshold value of about $0.2-1 \mathrm{~V}$, a rearrangement in the molecular structure of the membrane occurs, leading to the formation of pores and a substantial increase in the cell's permeability to ions and molecules. These last few decades, the popularity of the technique of electroporation has been increasing, notably for cell transfection [7-8]. However, the overall success rate of the process remains low: using batch electroporation typically only 40 to $70 \%$ of the cells are viably electroporated [9], most of them remain viable while being unaffected by the electrical stimulus and a small amount dies. Indeed, the whole process is difficult to control at the level of a cell population as the outcome of the electroporation process depends on a number of uncontrollable parameters, such as the cell size, shape and "vulnerability" [6].

One approach to increase the success rate of electroporation experiments consists of getting a better understanding of the mechanism(s) of pore formation and identifying keyparameters for this process through experimental work and/or theoretical modeling. Studies on the mechanism of pore formation provide insight into the pore location and into the sequence of molecular events leading to pore formation. For instance, the comparison between the electrical breakdown of cell membranes and model membranes has demonstrated that the pores originate in the lipid matrix of the membrane [10]. Furthermore, molecular dynamics simulations have demonstrated that the formation of a pore proceeds in three steps upon application of a voltage across the membrane. In the first stage, the electric field is locally enhanced, causing water defects in the bilayer structure. In the second stage, water molecules form a water file that spans the bilayer by establishing hydrogen bonds with each other. In the last stage, molecular rearrangement of the 
phospholipids in the vicinity of this water defect occurs and phospholipids molecules move towards this water channel to give a hydrophilic pore lined with phospholipid head groups [11-12]. Pores start to form nano- to microseconds after application of the electric field [1215], expand in a few milliseconds and close again in seconds to minutes in cells and milliseconds to seconds in artificial membranes [10, 14, 16-17]. The diameter of an electropore varies from 0.5 to $400 \mathrm{~nm}[12-13,15,18]$.

Another crucial aspect towards understanding the process of electroporation is the identification of key parameters affecting the stability of membranes. Experimental and theoretical studies have shown that this strongly depends on the composition of the membrane: the structural properties of the phospholipids found therein and the presence and amount of other membrane constituents. For instance, the electroporation threshold depends on the bilayer thickness, and consequently on the length of the phospholipid hydrocarbon chains [19-20]. Another key-factor is the presence of non-zero intrinsic monolayer curvature phospholipids in the membrane. Such lipids have a conical shape with either a large head group (positive intrinsic monolayer curvature) or a small head group (negative intrinsic monolayer curvature) compared to the hydrocarbon chains (see figure 4.1). These molecules cause packing defects in the membrane that facilitate the process of pore formation [21-22]. Interestingly, it has been demonstrated that the head group charge does not influence the membrane stability although one could expect that electrostatic interactions (repulsion or attraction) between different head groups would result in a change in the packing density of the membrane [19-20]. A second membrane compound of importance is cholesterol; it affects membrane properties not only through specific interactions with the hydrocarbon chains [23-27] but also because of its negative intrinsic monolayer curvature [21, 28-30]. Depending on the nature of the phospholipid molecules and the amount of cholesterol used this effect can be two-fold [24, 31-33]. The last main component found in natural membranes, peptides and proteins, also influences the stability although this phenomenon is still not poorly understood. For instance, Troiano et. al. showed that the presence of small peptides such as gramicidin $\mathrm{D}$ in the membrane decreases the probability of pore formation [34]. These results have been corroborated by recent molecular dynamics studies [35-36] and studies employing different proteins such as prestin [37].

Alternatively, external (natural or synthetic) molecules can also be employed to modulate the membrane properties: they strengthen molecular interactions between phospholipids or loosen the intermolecular structure of the membrane. For instance, both the detergent octaethyleneglycol mono n-dodecyl ether $\left(\mathrm{C}_{12} \mathrm{E} 8\right)$ [38] and the solvent dimethylsulfoxide (DMSO) [39] decrease the stability of the membranes by acting as positive curvature molecules. Additionally, the surfactant sodium dodecyl sulfate (SDS) reduces the membrane stability by lowering the interactions between phospholipid molecules due to its incorporation in the cell membranes [40]. On the other hand, the addition of the surfactant poloxamer 188 leads to an increase in the membrane stability; this is caused by either 
specific interactions with the phospholipids or by the formation of an insulating layer at the membrane surface, behaving as a shunting/screening layer for the applied voltage [41].
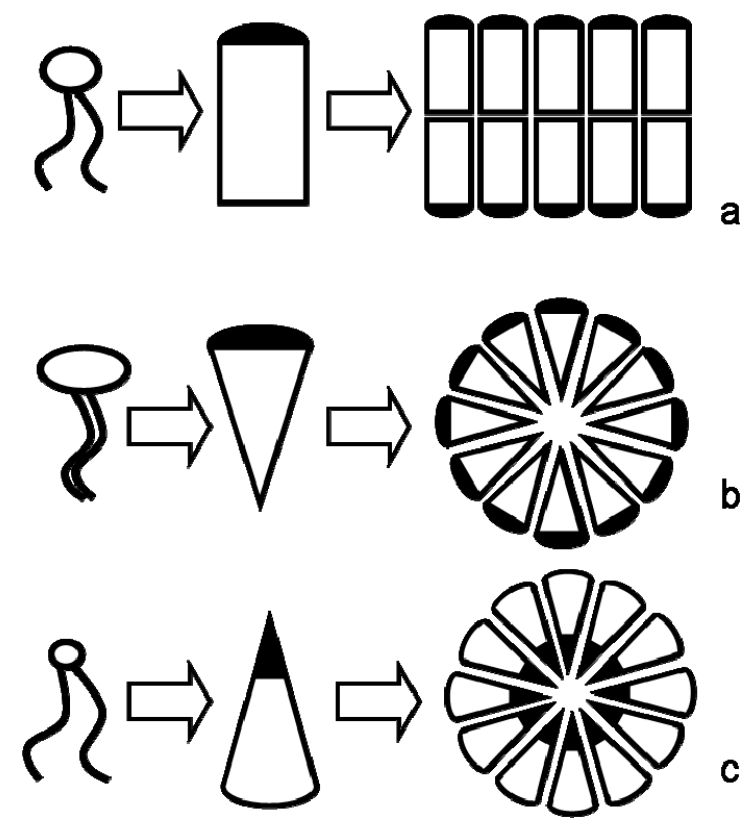

Figure 4.1. Phospholipid shapes

a) Phospholipid holding no curvature. These phospholipids will self-assemble into a bilayer structure. $b$ ) Phospholipid presenting a positive monolayer curvature. There phospholipids will prefer to assemble into micelles. c) Phospholipid having a negative intrinsic monolayer curvature. They will prefer to group into inverted micelles.

These different results suggest that the molecular aspects of the composition of the membrane are important for the creation of pores and electrical stability of the membranes, and that the interactions between the different molecules in the membrane are keyparameters in the process of pore formation.

In this chapter, we aim to get a better insight into the mechanism of electropore formation. For that purpose, we investigate the influence of the membrane composition and of its individual constituents on its (electrical) stability. Our approach consists of using artificial membrane models, bilayer lipid membranes (BLMs) and assessing the membrane stability by measuring the electroporation threshold. This threshold is defined as the potential at which pores are observed in the membrane. The basic "building block" we use to prepare the membranes is a synthetic phospholipid, 1,2-diphytanoyl-sn-glycero-3phosphocholine (DPhPC). This phospholipid is composed of two identical saturated hydrocarbon chains functionalized with methyl groups and a relatively large head group. It yields densely packed and very stable membranes. In a first stage, we investigate to which 
extent the electrical stability may be affected by the properties of the individual phospholipids present in the membrane. For that purpose, BLMs are prepared using a mixture of two phospholipids; DPhPC mixed with other phospholipids found in natural cell membranes that are unsaturated and have a non-zero intrinsic monolayer curvature. Following this, we examine how cholesterol affects pore formation in DPhPC membranes, and finally the contribution of one type of channel proteins, $\alpha$-hemolysin, on the same process.

\section{Materials and Methods}

\section{A. Chemicals}

Lipids (1,2-diphytanoyl-sn-glycero-3-phosphocholine (DPhPC), bovine heart L- $\alpha$ phosphatidylethanolamine (PE), bovine liver L- $\alpha$-phosphatidylinositol (PI), porcine brain L$\alpha$-phosphatidylserine (PS) and cholesterol (Ch) (Figure 4.2)) are purchased from Avanti Polar Lipids (Alabaster, AL). The protein $\alpha$-hemolysin is purchased from Sigma-Aldrich (St. Louis, $\mathrm{MO}$ ). $\mathrm{KCl}$, Hepes, Tris and n-decane are purchased from Sigma-Aldrich (St. Louis, MO). Chloroform is purchased from Merck Chemicals (Darmstadt, Germany). Deionized water $(18.2 \mathrm{~m} \Omega \times \mathrm{cm})$ which is used for all solution preparation and cleaning procedure is obtained using a MilliQ system (Millipore, Billerica, MA).

\section{B. Measurement set-up}

A conventional bilayer system (Warner Instruments, Hamden, CT) is used for BLM experimentation. This system comprises of a delrin cup and chamber containing two round compartments. The cup is inserted in the trans-compartment of the chamber, and the compartments are connected via a $150 \mu \mathrm{m}$ aperture in the cup across which BLMs are prepared. Both compartments contain $1 \mathrm{~mL}$ buffer solution and $\mathrm{Ag} / \mathrm{AgCl}$ electrodes (used for the electrical characterization of the BLMs). Electrical measurements are carried out with an Axopatch 200b amplifier (Molecular devices, Sunnyvale, CA), applying voltages and measuring currents across the bilayer. Data acquisition is performed with LabVIEW and a PCI-6259 data acquisition card (National Instruments, Austin, TX).

\section{Preparation and characterization of BLMs}

All lipids are used as chloroform-based solutions. DPhPC and PI are purchased at 10 $\mathrm{mg} / \mathrm{mL}$ solutions in chloroform. Other lipids (PS, PI, PE, and Ch) are purchased as powders and dissolved in chloroform to yield $25 \mathrm{mg} / \mathrm{mL}$ solutions. Before the preparation of BLMs, a few tenths of $\mu \mathrm{L}$ of the lipid solution in chloroform is left to evaporate overnight, yielding an amount of $500 \mu \mathrm{g}$ dried lipids, and subsequently dissolved in n-decane at a final concentration of $25 \mathrm{mg} / \mathrm{mL}$. To improve the solubility of the lipids 2-3\% ethanol can be added. The resulting n-decane solutions are used for the preparation of BLMs, and eventually mixed with other lipid solutions in case of multi-lipid membranes. 


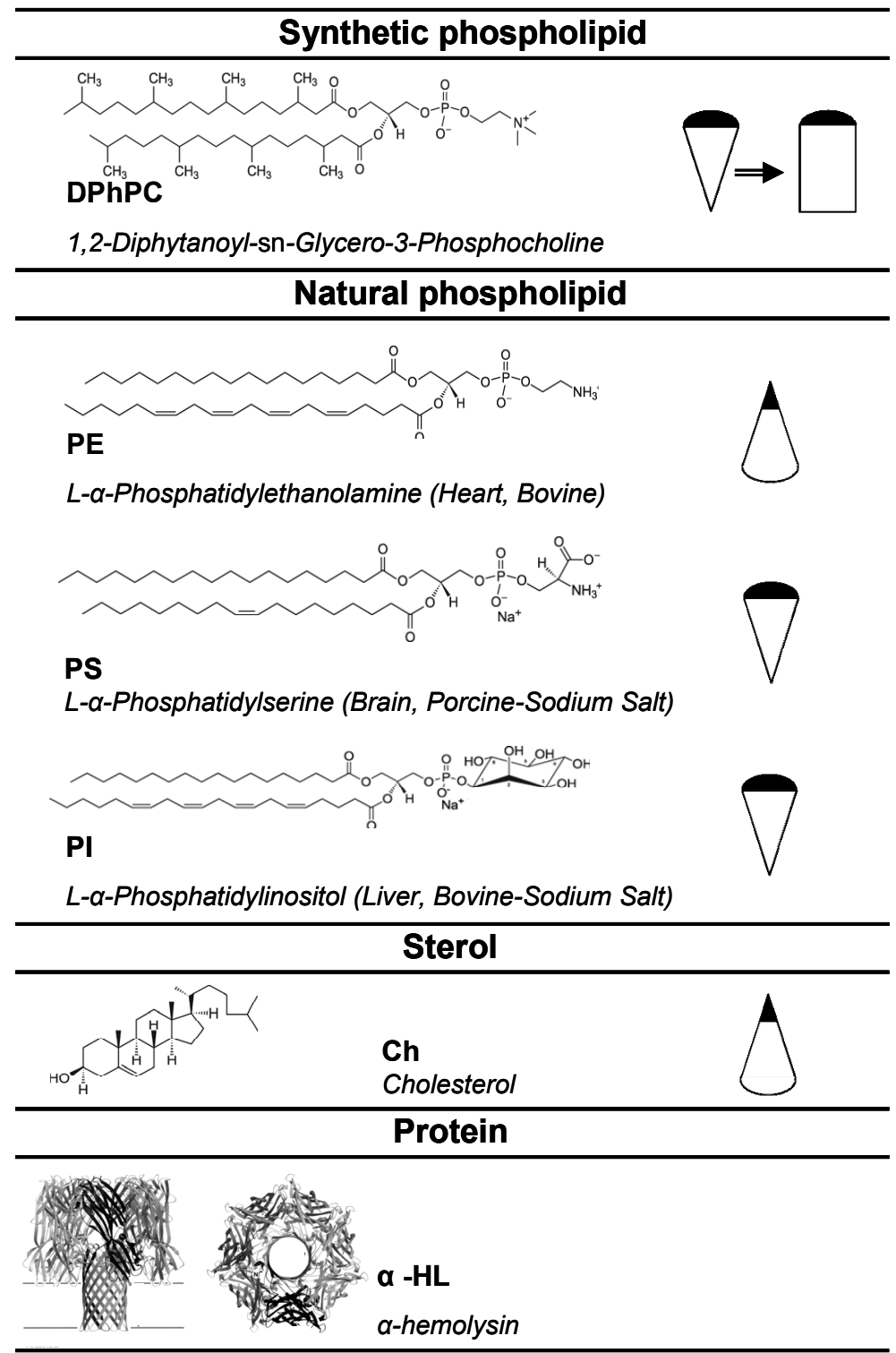

Figure 4.2. Molecular structures

Molecular structures of the phospholipids used in this study, cholesterol and the protein $\alpha$-hemolysin [42]. In the right column, the corresponding molecular shapes of the phospholipids and cholesterol are shown according to the classification of Israelachvili [29]. DPhPC can have two conformational shapes as explained in more detail in the text.

Two buffers are used for the BLM experimentation: a BLM buffer $(10 \mathrm{mM}$ Tris, $1 \mathrm{M} \mathrm{KCl}$, $\mathrm{pH}$ 8.5) for most experiments and an $\alpha$-HL buffer (5 mM Hepes, $1 \mathrm{M} \mathrm{KCl}, \mathrm{pH}$ 7.4) for 
experiments with $\alpha$-hemolysin as using the buffer of $\mathrm{pH} 8.5$ results in a noisy signal when $\alpha$ hemolysin molecules insert.

Bilayer lipid membranes are created using the Mueller-Rudin technique [43]. Bilayer formation is monitored using the Axopatch amplifier and characterized by a drop in the current due to the G $\Omega$ seal between the bilayer and the aperture. Thereafter, the membrane capacitance is measured as previously described [44] to confirm the formation of a BLM and as indication of the membrane properties; the membrane surface area is derived from the capacitance using equation 4.1 :

$$
A=\frac{C_{\text {mensured }}}{C_{\text {specific }}}
$$

where $A$ stands for the membrane surface area in $\mathrm{cm}^{2}, C_{\text {meas }}$ for the measured capacitance in $\mu \mathrm{F}$ and $C_{\text {specific }}=0.45 \pm 0.05 \mu \mathrm{F} / \mathrm{cm}^{2}$ [45].

\section{Insertion of protein channels in BLMs}

Alpha-hemolysin is dissolved in MilliQ water at a concentration of $2 \mathrm{mg} / \mathrm{mL}$. This initial solution is further diluted before any experiment to the desired concentration. $5 \mu \mathrm{L}$ of a diluted $\alpha$-hemolysin solution is added to $1 \mathrm{~mL}$ of the buffer ( $\alpha$-HL buffer) in the front compartment while stirring to promote protein diffusion to the membrane and their insertion. The insertion of individual proteins is monitored by recording the current. Every insertion gives rise to a characteristic jump in the current. After the insertion of a first protein, stirring is stopped as proteins continue inserting in the membrane.

\section{E. Determination of the electroporation threshold of BLMs}

The electroporation threshold $\left(V_{t h}\right)$ of the membrane is defined as the voltage at which the membrane conductivity increases due to the creation of pore(s). This value is determined as the value at which a leakage current is measured through the membrane. For that purpose, a DC voltage of $100 \mathrm{mV}$ is applied to the membrane using the Axopatch amplifier and gradually increased (every $30 \mathrm{sec}$ ) by steps of $20 \mathrm{mV}$, until peaks larger than three times the root mean-square of the noise are observed in the measured current. Pore formation is checked by re-applying the voltage at which peaks are detected; this confirms that the peaks are not due to imperfections in the membrane. The voltage value found corresponds to the electroporation threshold $V_{t h}$. For every membrane composition, $V_{\text {th }}$ is determined at least 3 independent times and the indicated value corresponds to the average of the three separate measurements.

\section{Results}

The ultimate goal of this study is to get a better insight into the influence of the molecular properties and the composition of the cell membrane on the mechanism of (electro)pore formation. For that purpose, we consider phospholipids, cholesterol and proteins found in natural cell membranes. The two main sections of this chapter focus on the lipidic formulation (phospholipid and cholesterol) and the protein content of the membrane. 


\section{A. Lipidic structure of membranes}

\section{(i) Phospholipid-phospholipid interactions}

In the first series of experiments we examine the interactions between different phospholipids placed in a membrane and their influence on the membrane's resistance to an applied electric field. We use membranes prepared from a mixture of two phospholipids, DPhPC and another phospholipid found in cell membranes (DPhPC-PE, DPhPC-PS, and DPhPC-PI), introduced in various weight percentages. We measure the membrane electroporation threshold $\left(V_{t h}\right)$ of the resulting membranes. Figure 4.3 (A-C) illustrates the variations of the measured electroporation threshold $V_{\text {th }}$ when the membrane composition was varied from $100 \%$ DPhPC membranes to $100 \%$ of the second phospholipid.

Some trends are apparent in these variations depending on the added phospholipid molecule. The differences in the membrane stability can be explained by a change in the packing density of the phospholipid molecules. These are induced by the unsaturations present in the hydrocarbon chains of the phospholipids or by their non-zero intrinsic monolayer curvature. Firstly, phospholipids that contain unsaturations in the chain are forced further apart due to kinking of the hydrocarbon chains [2]. Secondly, according to the classification proposed by Israelachvili [29], the packing density of phospholipids correlates with their shape: cylindrical, tapered or frayed (See Figure 4.2). The two latter groups display a non-zero intrinsic monolayer curvature (negative or positive, respectively) and the individual molecules prefer to assemble as micellar structures. As a consequence, their presence in a planar membrane results in defects in the molecule packing [21,39], a decrease in the membrane stability upon application of an external voltage and a facilitated pore formation. These two factors (the eventual presence of unsaturations and the intrinsic monolayer curvature of the phospholipid molecules) correspond well to the observed trends in $V_{\text {th }}$ for the three systems considered here.

DPhPC possess two identical saturated hydrocarbon chains functionalized with four methyl groups (in positions 3, 7, 11 and 15) and a relatively large head group (see Figure 4.2). DPhPC molecules are slightly tapered, but once they are introduced in a planar membrane they tend to adopt a cylindrical shape by the formation of interdigitated structures by the methyl-functionalized chains [29, 46-47]. As a consequence, the lateral and rotational motion of individual phospholipid molecules is dramatically decreased compared to other PC species [48]. DPhPC forms highly packed and ordered membranes, reflected by their low surface tension (32-37 vs. $54-56 \mathrm{mN} / \mathrm{m}$ for most of the phospholipids) [49] and they are in the liquid crystal phase (between -120 and $120^{\circ} \mathrm{C}$ typically). The addition of other (phospho)lipid species in the membrane breaks the DPhPC interdigitated network, allowing molecules to move freely and subsequently yielding less stable membranes.

PE (L- $\alpha$-Phosphatidylethanolamine) was first added in DPhPC membranes. As shown in Figure 4.3A, we find a linear decrease in the electroporation threshold upon the progressive introduction of PE in DPhPC membranes: from $204 \pm 16 \mathrm{mV}$ for $100 \% \mathrm{DPhPC}$ 
membranes to $160 \pm 28 \mathrm{mV}$ for $100 \%$ PE membranes. This marked decrease in $V_{\text {th }}$ is accounted for by both the structural properties of PE molecules and the destruction of the DPhPC intertwined network. The predominant species of the used PE holds four unsaturations (see Figure 4.2) so its addition leads to a progressive loosening of the phospholipid packing [2]. Moreover, PE presents a slight conical shape due to its smaller head group where methyl groups are replaced by hydrogen atoms (see Figure 4.2). It displays a negative intrinsic monolayer curvature [21, 29] and is a non-bilayer preferring lipid under physiological conditions [50]. As a result, PE-based membranes are reported as highly porous [51] with a large amount of packing defects [21]. We can conclude that the linear decrease we see in the electroporation threshold is caused by the sum of three factors: the loss of the intertwining network, the progressive addition of unsaturations and the introduction of packing defects in the membrane [21, 50-51].

A similar trend in the variations of $V_{\text {th }}$ was expected for DPhPC-PS systems as the predominant species of the used PS (L- $\alpha$-phosphatidylserine) presents one unsaturation, a large head group and consequently a positive intrinsic monolayer curvature. Interestingly, although we measure a lower electroporation threshold for 100\% PS membranes (168 \pm 39 $\mathrm{mV}$ against $204 \pm 16 \mathrm{mV}$ for $100 \%$ DPhPC membranes), the variations of $V_{\text {th }}$ do not follow a monotonous trend upon addition of PS. They resemble the shape of a bell (see Figure 4.3B), and $V_{\text {th }}$ reaches the maximum value of $290 \pm 26 \mathrm{mV}$ around 2:1 DPhPC:PS membrane. Firstly, as for PE, the addition of PS results in a decrease of the phospholipid packing density due to the loss of the chain entanglement and the addition of unsaturations. Furthermore, packing defects are created in the membrane since PS has a positive intrinsic monolayer curvature. However, for low amounts of PS, another phenomenon must counteract these three factors to explain the increase in $V_{\text {th }}$ we observe for PS amounts up to around $33 \%$. We attribute this stabilization parameter to electrostatic interactions taking place between the negative charge found in the head group of PS and the zwitterionic head group of DPhPC, as illustrated in Figure 4.3D. While it is known from literature that the phospholipid charge does not affect the membrane stability and the process of pore formation [19-20], we hypothesize that this charge interaction strengthens the membrane. As a summary, four contributions are found for DPhPC-PS systems; the same three destabilizing effects as for DPhPC-PE membranes and a counteracting electrostatic stabilizing effect, resulting in the observed bell-shaped variation of $V_{t h}$.

Comparable behaviour is demonstrated for PI (L- $\alpha$-phosphatidylinositol) which correlates well to the facts that the predominant species of the used PI and PS both present unsaturations (four in the case of PI), a positive intrinsic monolayer curvature and a negative charge (see Figure 4.2). As shown in Figure 4.3C, $V_{\text {th }}$ first remains more or less stable (up to $~ 33 \% \mathrm{PI}$ ), and subsequently strongly decreases to reach a value of $92 \pm 33 \mathrm{mV}$ for mono-PI membranes. This value is notably smaller than the $V_{\text {th }}$ observed for $100 \%$ PE and PS membranes. The same four contributions are found for PI and PS: three destabilizing contributions (destruction of the DPhPC network, addition of unsaturations and a positive 
intrinsic monolayer curvature) and one stabilizing contribution (charge interactions between PI and DPhPC head groups (see Figure 4.3D)). However, this last stabilizing contribution is quickly overcome by the other opposite factors, most probably due to the higher number of unsaturations found in PI and the larger head group. This translates into the observed plateau in $V_{\text {th }}$ for PI amounts lower than $\sim 33 \%$, instead of an increase.
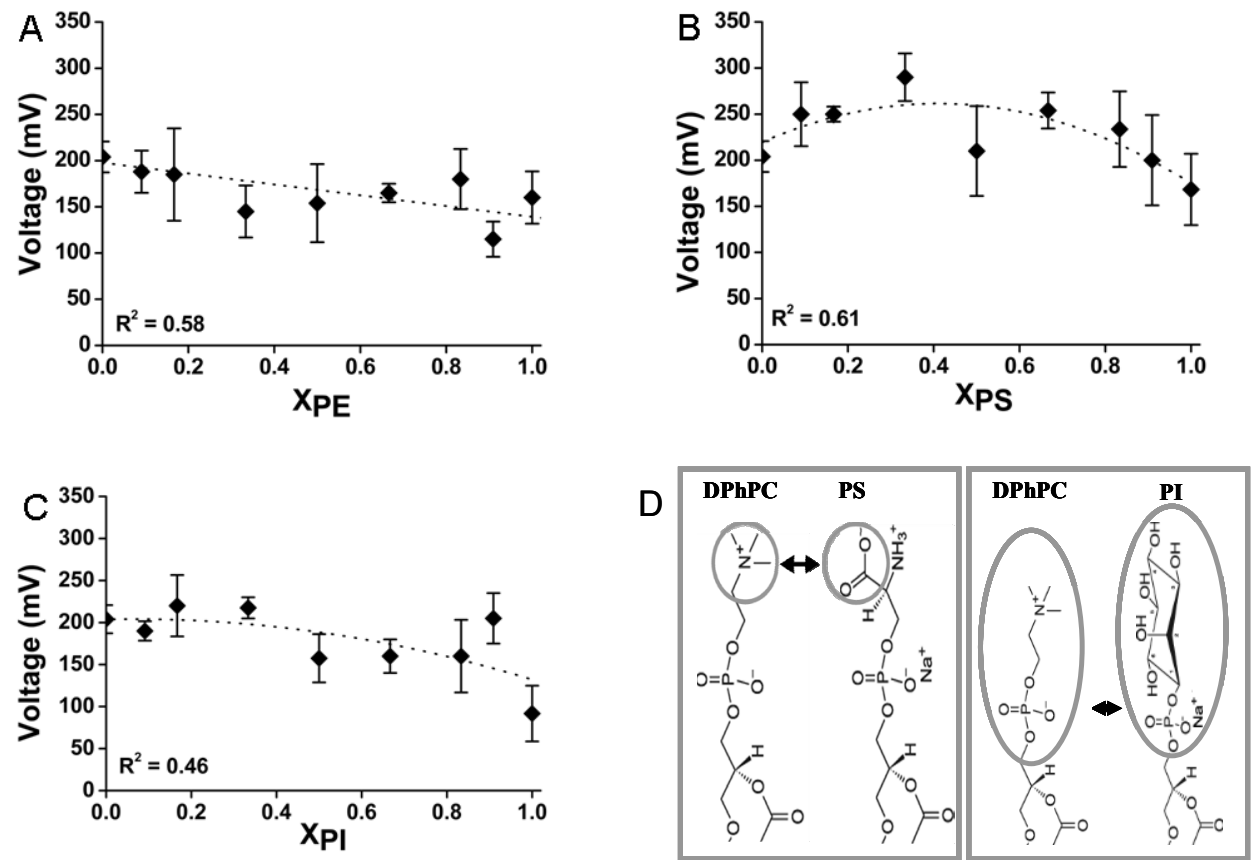

Figure 4.3. Results for the phospholipid-phospholipid interactions

(A-C) Electroporation threshold $V_{\text {th }}$ measured for bi-phospholipid BLMs plotted as a function of the weight percentage of the second phospholipid. The BLMs are prepared using mixtures of DPhPC-PE (A), DPhPC-PS (B) and DPhPC-PI (C). Dotted lines represent the best fit for the data derived in Origin (OriginLab, Northampton, $M A)$. (D) Illustration of the charge interactions taking place between the negative head groups of PS (left) and PI (right) and the zwitterionic head group of DPhPC.

These three series of experiments confirm that the chemical and structural properties of individual phospholipids influence the membrane stability and the creation of pores upon application of an electric field. Depending on the molecular properties of the phospholipids this effect can be either stabilizing or destabilizing. These results also confirm that more than one type of interactions is interfering with the pore formation process.

\section{(ii) Phospholipid-cholesterol interactions}

Another lipidic parameter we consider here is cholesterol, found in natural membranes at up to $14 \%$ weight (equivalent to $30 \% \mathrm{~mol}$ ) [1,52]. The variations of the electroporation 
threshold $V_{\text {th }}$ for membranes containing $0-20 \%$ weight cholesterol clearly present two different trends (see Figure 4.4). At low cholesterol concentrations (1-5\%), we observe a progressive increase in $V_{\text {th }}$ that reaches the value of $254 \pm 13 \mathrm{mV}$ for $5 \%$ weight cholesterol. At higher cholesterol concentration, the tendency is inversed, and the addition of cholesterol leads to a decrease in $V_{\text {th }}$ down to $104 \pm 52 \mathrm{mV}$ for $20 \%$ cholesterol. Interestingly, this final value of $V_{\text {th }}$ is approximately half of the value measured for $100 \%$ DPhPC membranes (204 \pm $16 \mathrm{mV})$.

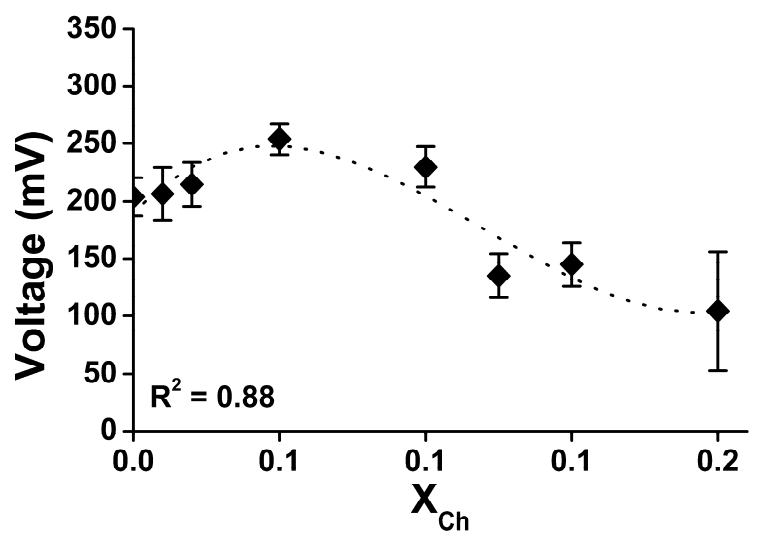

Figure 4.4. Result for the phospholipidcholesterol interaction

Electroporation threshold $V_{\text {th }}$ measured for DPhPC-Ch membranes. $V_{\text {th }}$ is plotted against the weight percentage $(0-20 \%)$ of cholesterol in the membrane. The dotted line represents the best fit for the data derived in Origin (OriginLab, Northampton, MA).

In our experiments, we observe two opposite effects: (i) a stabilization of DPhPC membranes at low cholesterol amounts followed by (ii) a destabilization for cholesterol amounts higher than $5 \%$. These tendencies are explained, as for the former experiments with bi-phospholipid systems, by a combination of different parameters that affect the molecular packing density.

The well-known function of cholesterol is to regulate the permeability and the properties of the membranes of mammalian cells [1-2]. This is achieved through various effects on the phospholipid molecules, ordering or condensing effects and the establishment of specific interactions with the phospholipids [1]. The presence of cholesterol subsequently results in (i) an increase in the order and the density of the phospholipid network [29, 53], (ii) thickening of the membranes [54] and (iii) a reduction in the mobility of the phospholipid molecules [30,54]. These factors consequently disfavour the deformation of the bilayer and pore formation [24, 33, 54]. Interestingly, the effect of cholesterol depends on the structural properties of the phospholipids, and specifically occurs with saturated phospholipids [29, 50] and lipids having a high melting temperature $\left(T_{m}\right)$ [55]. However, while DPhPC is saturated, it has a low $T_{m}\left(<-20^{\circ} \mathrm{C}\right)$ [49] and it has been reported to have a similar effect on membranes as cholesterol [48]. As a consequence, DPhPC membranes are already densely packed and rigid. This density found in DPhPC membranes is accounted for by the formation of an intertwined network with neighbouring hydrocarbon chains 
being interdigitated [46-47]. Still, we assume that not all hydrocarbon chains are involved in this network and that there is some room for a few cholesterol molecules to insert into the membrane, bind to DPhPC molecules and condense the phospholipid network. This results in a further rigidification and stabilization of the membrane, and yields an increased resistance to the application of an electric field, as observed for cholesterol amount up to $5 \%$ weight.

For higher cholesterol amounts, the membrane stability decreases. As already mentioned, there is little free space for cholesterol to insert into the dense network formed by $\mathrm{DPhPC}$ molecules, so further addition of cholesterol translates into the disentanglement of the hydrocarbon chains of DPhPC molecules and the subsequent rupture of the intertwined network. The membrane becomes less dense and cholesterol stops behaving as a condensing molecule but starts acting as a curvature-inducing molecule in the same manner as PE [21], further destabilizing the membrane. Consequently, above $5 \%$ cholesterol in the $\mathrm{DPhPC}$ membranes the progressive decrease of the electroporation threshold we measure is due to the rupture of the dense DPhPC network and the addition of a negative intrinsic monolayer curvature molecule, as observed for PE. Interestingly, the destabilizing effect of cholesterol is much higher than for PE. $20 \%$ weight of cholesterol in a DPhPC membrane leads to a $V_{\text {th }}$ of $104 \pm 52 \mathrm{mV}$ against $160 \pm 28 \mathrm{mV}$ for $100 \%$ PE membranes. An explanation for this effect is that cholesterol is a rigid planar molecule, whereas PE is deformable. Cholesterol subsequently introduces larger defects in the mixed membrane and thereby facilitates pore formation upon application of an electric field.

\section{B. Proteins}

In the coming section, we consider the influence of another essential constituent of cellular membranes, proteins. We investigate not only the effect of the presence of proteins, but also that of the amount of proteins in a BLM. We use a well-studied protein channel, $\alpha$ hemolysin, that fairly easily incorporates in BLMs, as a model for membrane proteins. As its pore is always in the open state $[42,56-57]$, the introduction of every pore generates a leakage pathway in the membrane and protein insertion can easily be identified by the apparition of characteristic jumps in the trans-membrane current. The insertion of every protein corresponds to a jump in the current of $\sim 50 \mathrm{pA}$ upon application of a DC voltage of $50 \mathrm{mV}$ across the membrane as the channel has a $1 \mathrm{nS}$ conductance when a buffer of $1 \mathrm{M} \mathrm{KCl}$ is employed [58].

\section{(i) Monitoring the protein density}

Most experiments on proteins in BLMs focus on getting as few proteins as possible in these membranes enabling the study of individual proteins [59]. As we want to simulate real cell membrane conditions where proteins represent $50 \%$ of the weight of the membranes, experimentation with only a few proteins in the BLMs is not representative. Therefore, we adopt a more comprehensive approach and endeavour to monitor the amount of proteins we introduce in DPhPC membranes: from a single protein per membrane to a weight 
percentage of $\sim 50 \%$, to come as close as possible to a real-world situation. This differs from similar experiments found in the literature where small peptides (gramicidin A) that are able to form channels across the membrane are used, and the peptide is added in a known concentration to the lipid mixture before making the membranes [34]. We observe here the insertion of individual proteins in an already prepared DPhPC membrane.

The results of the experiments are summarized in Table 4.1. When the concentration of $\alpha$-hemolysin in the reservoir is $2.5 \times 10^{-2} \mu \mathrm{g} / \mathrm{mL}$, we get dynamics of insertion of 1 protein every 10 minutes. This implies that if the buffer is refreshed after the first insertion, experiments at the single protein level are conceivable. When raising the $\alpha$-hemolysin concentration in solution to 5 or $10 \times 10^{-2} \mu \mathrm{g} / \mathrm{mL}$, insertion proceeds much faster, with typically $3-10$ or $60-100$ proteins per 10 minutes, respectively (see Figure 4.5 ). Subsequently, by varying the protein concentration in solution and by refreshing the buffer solution after 10 minutes, we are able to modulate the protein density in the membrane from $0.11 \times 10^{-3}(1$ protein in the membrane) to $11 \times 10^{-3}$ proteins $/ \mu \mathrm{m}^{2}(\sim 100$ proteins in the membrane $)$ for membranes having an average surface area of $\sim 9 \times 10^{3} \mu \mathrm{m}^{2}$. We will discuss these density values with respect to real-world situations in the discussion.

\section{Table 4.1. Dynamics of insertion of $\alpha$-hemolysin}

Dynamics of insertion of $\alpha$-hemolysin as a function of the protein concentration in solution: number of proteins inserted in the membrane every 10 minutes and corresponding protein densities (for a membrane with a given surface area of $\sim 9 \times 10^{3} \mu \mathrm{m}^{2}$ )

\begin{tabular}{|c|c|c|}
\hline $\begin{array}{l}\text { Protein concentration in solution } \\
\qquad(\mu \mathrm{g} / \mathrm{mL})\end{array}$ & $\begin{array}{l}\text { Dynamics of insertion } \\
\text { (\# proteins/10 minutes) }\end{array}$ & $\begin{array}{c}\text { Protein density } \\
\left(\times 10^{-3} \text { proteins } / \mu \mathrm{m}^{2}\right)^{\mathrm{b}}\end{array}$ \\
\hline $2.5 \times 10^{-2}$ & 1 & 0.11 \\
\hline $5 \times 10^{-2}$ & $3-10$ & $0.33-1.1$ \\
\hline $10 \times 10^{-2}$ & $60-100$ & $6.8-11$ \\
\hline
\end{tabular}

a Since one jump of $\sim 50 \mathrm{pA}$ corresponds to the insertion of one protein, the amount of proteins is determined using the total current increase.

${ }^{b}$ The protein density is calculated by dividing the total amount of proteins by the membrane surface area. The membrane surface area is calculated using equation 4.1 and an average measured capacitance of $40 \mathrm{pF} \pm 11 p F$.

\section{(ii) Phospholipid-protein interactions}

Since we are able to monitor the insertion of $\alpha$-hemolysin in BLMs, we can characterize the membrane stability not only as a function of the presence of proteins but also as a function of their density in the membrane. First of all, although we are able to insert a large amount of proteins in the membranes (>6000 proteins), it is not possible to conduct electroporation experiments with a lot of proteins due to the patch-amplifier employed for the experiments and the buffer concentration chosen $(1 \mathrm{M} \mathrm{KCl})$. As mentioned, one protein has a conductance of $1 \mathrm{nS}$, every protein gives rise to a leakage current whose amplitude depends on the voltage applied on the membrane. Electroporation studies start with a 
voltage of $200 \mathrm{mV}$, which gives a total leakage current of $100 \mathrm{nA}$ if 500 proteins are present in the membranes, while the measurement range of the patch-amplifier is restricted to $198 \mathrm{nA}$. Consequently, for more than 990 proteins, the current saturates before the electroporation experiments can be performed.

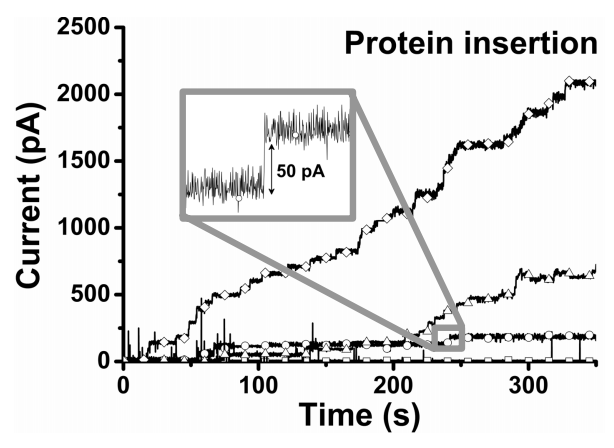

Figure 4.5. Monitoring the protein insertions Monitoring the insertion of $\alpha$-hemolysin in DPhPC membranes. Leakage current measured across a DPhPC membrane as a function of the time upon application a $50 \mathrm{mV}$ voltage (1 $\mathrm{M} \mathrm{KCl}$ buffer). Protein concentrations of $2.5 \times 10^{-3} \mu \mathrm{g} / \mathrm{mL}$ (squares), $5 \times 10^{-3}$ $\mu \mathrm{g} / \mathrm{mL}$ (circles), $10 \times 10^{-3} \mu \mathrm{g} / \mathrm{mL}$ (triangles) and $25 \times$ $10^{-3} \mu \mathrm{g} / \mathrm{mL}$ (diamonds). The jumps in the current correspond to the insertions of individual proteins (see inset) as the insertion of one protein corresponds to an increase of $50 \mathrm{pA}$ in the current (the conductance of a single $\alpha$-hemolysin protein is $1 \mathrm{nS}$ ).

For the range where measurements could be carried out (i.e. until $\sim 1000$ proteins), $V_{\text {th }}$ is measured as a function of the amount of proteins inserted in the membranes. In that range, no real change of $V_{\text {th }}$ is detected. It remains equal to $200 \mathrm{mV}$, as observed for membranes without proteins (see figure 4.6).

\section{Discussion}

We have studied the effect of three components found in natural cell membrane on the stability of planar mimics of membranes based on the DPhPC phospholipid: (i) the chemical and structural properties of various phospholipids, (ii) the effect of cholesterol and (iii) the influence of proteins ( $\alpha$-hemolysin). These three factors affect the stability of the membrane, and subsequently the probability to form pores in a membrane upon application of an electric field.

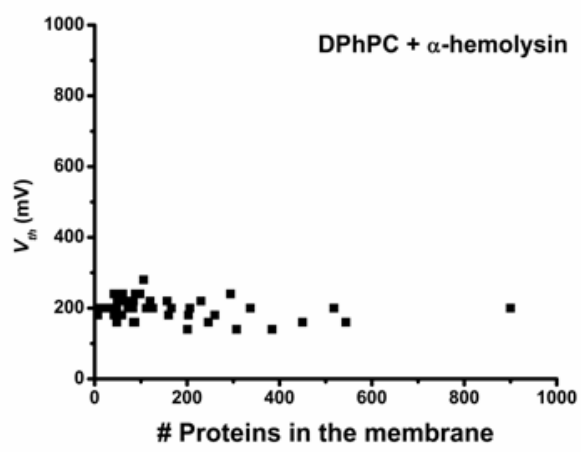

Figure 4.6. Results for phospholipid-protein interactions

Electroporation threshold $V_{\text {th }}$ of $\alpha$-hemolysin containing DPhPC membranes plotted against the amount of proteins introduced in the membrane. The black squares represent individual measurements of $V_{\text {th }}$ for a given amount of proteins. 


\section{A. Lipidic contributions}

The phospholipid composition has a low contribution on the membrane stability. However, we can still see clear trends in $V_{\text {th }}$ variations related to the different chemical and structural phospholipid properties and particularly the subsequent packing density of the individual molecules. On one hand, the packing density of the phospholipids is increased when specific interactions are established between the molecules. This is illustrated with the condensing effect observed with cholesterol [52] but also to a less extent when electrostatic interactions take place between head groups of opposite charges (zwitterionic and negative). In both cases, the membrane becomes more stable and has a stronger resistance to the application of an electric field. On the other hand, the packing density becomes loosened when unsaturated or non-zero intrinsic monolayer curvature phospholipids are used. The use of non-zero intrinsic monolayer curvature phospholipids results in bending of the membrane and the creation of packing defects while unsaturations tear phospholipid molecules apart.

While the mechanism of electropore formation is not yet fully elucidated, different models proposed from molecular dynamic simulation studies give insight into this process $[12-13,36]$ and highlight how the packing density or pattern of the phospholipids affect the process of pore formation. As already mentioned in the introduction and described in more detail in chapter 2, pore formation proceeds in three steps, (i) a local enhancement of the electric field and the creation of water defects, (ii) the formation of a water file through the membrane and (iii) the rearrangement of the phospholipid molecules to create a hydrophilic pore [12], which are favoured or disfavoured depending on the phospholipid packing pattern, as explained below.

\section{(i) Local enhancement of the electric field and the creation of water defects.}

This first step is favoured when packing defects are present in the membrane. DPhPC membranes are very stable and well-packed membranes [46-48]; there is little or no defect and little chance for insertion of water molecules. As a result, the probability for pore formation remains very low. The addition of non-zero intrinsic monolayer curvature phospholipids (PI, PE, PS) causes the amount of defects to increase [21], enabling local enhancement of the electric field and facilitating the insertion of water molecules.

\section{(ii) Formation of a water file through the membrane, or creation of a hydrophilic} pore.

Water molecules can move more easily into less densely packed membranes [12] to form a file through the membrane or a hydrophilic pore once a water defect is created. In case of DPhPC membranes, this step is again not likely as the creation of a water line requires the rupture of the intertwined network formed by the hydrocarbon chains. Similarly, if electrostatic interactions take place between phospholipid head groups (DPhPC/PI or DPhPC/PS) these interactions must be broken for water molecules to span the membrane. On the other hand, unsaturated phospholipids are less densely packed giving 
space for water molecules to insert in the membrane and create a water file, increasing the likelihood of the second step of pore formation.

\section{(iii) Rearrangement of the phospholipid molecules to create a hydrophobic pore.}

The last step in the process of pore formation is the rearrangement of the phospholipid molecules to line the water file and create a hydrophilic pore. In general, this last step is favoured with non-zero curvature lipids. The largest effect is still observed for lipids having a positive intrinsic monolayer curvature (PS, PI), those prefer to assemble as micellar structures and the edge of the pore resembles a semi-micelle (see Figure 4.7A). In that case, a bulkier headgroup would result in a larger intrinsic monolayer curvature and a more marked stabilization effect. PI has a larger head group than PS, and thus a larger intrinsic monolayer curvature, so it will favour this step more than PS. Interestingly, lipids presenting a negative intrinsic monolayer curvature such as PE also contribute to pore stabilization but more for small pores: these lipids would line and circumvent the pore in the plane of the bilayer membrane [60], as represented in Figure 4.7B. Again, the pore seen from the top resembles an inverted micelle, the preferred structure for those phospholipids. As the curvature of the pore in the plane of the membrane is larger for smaller pores, this stabilizing effect is more important for smaller pores than for larger ones for which the curvature will be negligible.
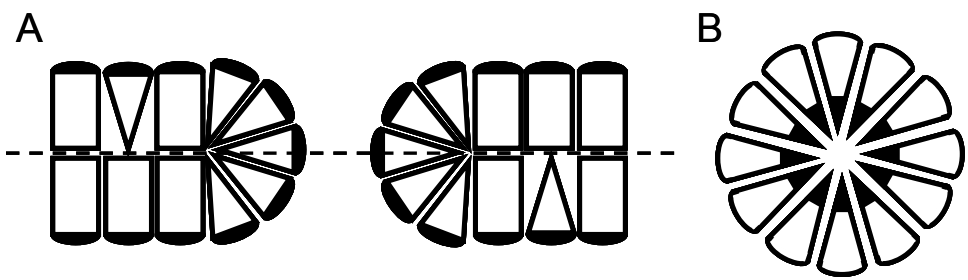

Figure 4.7. The effect of the intrinsic monolayer curvatures

Schematic showing of the effect of the intrinsic monolayer curvatures of the phospholipids on the last step of the pore formation process during electroporation; the lining of the pore by the phospholipid head groups. A) Side view of a pore in a bilayer membrane containing zero and positive intrinsic monolayer curvature phospholipids. Phospholipids having a positive intrinsic monolayer curvature will prefer to line the edge of the pore along the normal of the bilayer as it resembles a semi-micellar structure. B) Top view of a pore in the middle of the bilayer plane. The structure of the pore seen from the top resembles an inverted micelle; consequently, phospholipids having a negative intrinsic monolayer curvature prefer to surround the pore in the plane of the bilayer (indicated by the dotted line in (a)) and stabilize it.

As a conclusion, all three steps are disfavoured in DPhPC membranes, and so is the creation of electropores. PE will favour the two first steps and the third step only in a poresize dependent manner. Globally, its presence in the membrane has only a slight influence on the electroporation threshold (160 against $200 \mathrm{mV}$ for DPhPC membranes). PI and PS favour all three steps, but the second step to a lesser extent as the presence of unsaturations is counterbalanced by the existence of electrostatic interactions between the head groups. 
Finally, as PI presents 4 unsaturations and a larger head group, its contribution to pore formation is higher. All together, the probability of pore formation would increase in the following order DPhPC $<$ PE $<$ PS < PI for the bi-phospholipidic systems we consider here, and this is in good agreement with our observations.

\section{B. Cholesterol}

Cholesterol gives an interesting and concentration-dependent two-fold effect in the 0$20 \%$ weight range. These results are nonetheless specific to our phospholipidic system as $\mathrm{DPhPC}$ forms an extremely dense and stable membrane, and they are expected to be different for other phospholipid species.

The natural role of cholesterol is to stiffen and order the membrane by strengthening the interactions between individual phospholipids forming the membrane [1, 52]; this conformational order makes the membrane more resistant to external stress, increases its stability and lowers its permeability to water and ions. In presence of cholesterol, the energetic cost to create water defects in natural membranes is dramatically increased, and cholesterol will prevent the creation of defects and water pores in the membrane [54]. In addition, the presence of cholesterol mostly induces thickening of the membrane, depending on the length of the phospholipid hydrocarbon chains [54].

However, we use DPhPC as basic units to prepare our membranes, and this belongs to the family of archaeal lipids [48-49]. This phospholipid is not found in natural membranes of mammalian cells but in those of archaebacteria that must resist harsh conditions such as high temperatures [49]. As a consequence, archaeal lipids must confer strong barrier properties and great stability to the membranes, as cholesterol would do for mammalian cell membranes. The effects of DPhPC are indeed similar to those of cholesterol [48]. DPhPC gives high molecular ordering of the membrane; it limits the trans/gauche conformation of the phospholipid molecules and their lateral and rotational motion while enhancing the motion of head groups (tilting). Finally, in both systems, a larger distance is found between head groups and subsequently, less head group interactions, either due to dominating cholesterol-phospholipid interactions or to the larger molecular area accounted by for the branched acyl chains [48].

It would be interesting to mix cholesterol to another phospholipid found in natural systems to be able to compare the results better to the outcome of mammalian cell electroporation experiments. In natural membranes, cholesterol is mainly present in stable microdomains found in the membrane, known as lipid rafts [61-62]. These domains are composed of a mixture of glycerolipids, sphingolipids and cholesterol. Therein, cholesterol prefers to bind to sphingolipids for shape reasons as sphingolipids are mostly saturated while glycerolipids present at least one unsaturation in natural systems. Future experiments with cholesterol should also take into account these microdomains, notably with the introduction of sphingolipids in our membranes and the study of ternary systems based on a mixture of glycero-, sphingolipids and cholesterol. 


\section{Proteins}

Our double goal for the experiments with proteins was to be able to control their density in the membrane and to assess how the membrane stability would be affected by different amounts of proteins. For that purpose, our idea was to be able to cover a wide range of protein density, from the single protein level up to a situation close to what is found in natural membranes, i.e. a $50 \%$ weight amount of proteins. To our best knowledge, no comparable study on the electrical stability of membranes depending on the concentration of channel proteins is reported in literature. Existing studies on the concentration dependency are limited to small peptides such a gramicidin D that can form transient pores, and these peptides were directly introduced in the lipid mixture in a known ratio [34], while we monitor their individual insertion in the membrane. Navarette et al. studied the effect of the motor protein prestin found in the lateral plasma membrane of outer hair cells (OHCs) on the susceptibility to electroporation of these cells or transfected cells homogeneously expressing the protein [37].

In this study, we benefit from the fact that $\alpha$-hemolysin is an open channel protein with a $1 \mathrm{nS}$ conductance in a $1 \mathrm{M} \mathrm{KCl}$ solution [58] and the insertion of a single protein results in a leakage current. We use a $50 \mathrm{mV}$ voltage signal to make the insertion easy to detect from the noise of the signal, and every protein insertion gives rise to a $50 \mathrm{pA}$ leakage current. The presence of 360 proteins in the membranes consequently leads to a leakage current of $18 \mathrm{nA}$ which is the maximum current we can measure with our patch amplifier. As a consequence, in this configuration we are limited to a protein range of 1-360 proteins per membrane. To overcome this, the protein amount in the membrane can be measured afterwards using another method; employing the patch-amplifier mode used to detect the formation of the $\mathrm{G} \Omega$ seal during membrane preparation. This mode uses a low $1 \mathrm{mV}$ voltage. Thereby, every protein gives rise to a leakage current of $1 \mathrm{pA}$ allowing us to measure the presence of up to 18,000 proteins in the membrane. Nonetheless, this method is more suitable for measuring large numbers of proteins afterwards and not to monitor the insertion of individual protein as the $1 \mathrm{pA}$ leakage current given by a single protein may be difficult to distinguish from the signal noise.

The protein density is subsequently calculated from the total number of proteins inserted in the membrane and using the surface area of the membrane $\left(\sim 9 \times 10^{3} \mu \mathrm{m}^{2}\right)$ assessed from a capacitance measurement (see section 2.3). Until now we have achieved protein densities of up to 0.74 proteins $/ \mu \mathrm{m}^{2}$ (6500 proteins). For a comparison, a cell membrane contains a protein density of $1.25 \times 10^{5}$ proteins $/ \mu \mathrm{m}^{2}$ as $50 \%$ weight of proteins in a membrane corresponds to a $\sim 40: 1$ molar ratio between phospholipids and proteins. As the molecular weights of DPhPC and $\alpha$-hemolysin are 0.85 and $33 \mathrm{kDa}$ and a cell membrane contains approximately $5 \times 10^{6}$ phospholipids/ $\mu \mathrm{m}^{2}$ [2], a "natural" protein density in our model would be $1.25 \times 10^{5}$ proteins $/ \mu \mathrm{m}^{2}$. This is $\sim 5-6$ orders of magnitude higher than what we have achieved until now. An option to reach a higher protein density is to use smaller membranes. The advantage of using smaller membranes is that the smaller the membrane, 
the lower the noise level and the more sensitive the measurements, so that protein insertion could simply be followed by applying a $1 \mathrm{mV}$ voltage. In that case, a "natural" protein density can be reached for instance by using a $\sim 0.12 \mu \mathrm{m}^{2}$ membrane for 15,000 proteins inserted, which is achievable with a $0.5-1 \mu \mathrm{m}$ diameter aperture.

Originally, an increase in $V_{\text {th }}$ was expected as a function of the amount of proteins present in the membrane, as reported for gramicidin by Troiano et al. [34]. They describe an increase in the electroporation threshold of 16 and $40 \%$ when the small peptide gramicidin was added in a 1:500 and 1:15 peptide:phospholipid molar ratio, respectively. Furthermore, at a lower peptide concentration (1:10,000 molar ratio) the electroporation threshold remained unchanged. The experimental results of Troiano et al. have later been corroborated by molecular dynamics simulations, showing a significant reduction in pore formation in membranes containing gramicidin A peptides [35]. These simulations provide a model for the influence such a small peptide would have on a membrane and its properties; it locally change the thickness of the membrane and by consequence, its resistance to the application of an electric field. Similarly, Navarrete et al. found a change in the mechanical properties and the stiffness of the membrane in the presence of a protein, prestin [37]. Here, another pore-forming entity is used, $\alpha$-hemolysin. If the presence of a small peptide, gramicidin, affects the mechanical properties of membranes, a largest influence is to be expected for $\alpha$ hemolysin which is bigger than gramicidin ( $30 \mathrm{kDa}$ vs. $3.76 \mathrm{kDa})$. In addition, while the electrical contribution for gramicidin can be excluded, the pore of $\alpha$-hemolysin is bigger. Therefore, the presence of an $\alpha$-hemolysin pore in the membrane results in a local drop in the membrane potential ( $4 \%$ at the mouth of the pore), and this lowers the probability for membrane poration. This goes in the same direction as the standard theory for pore formation, which predicts that the existence of a pore in an area of the membrane diminishes the probability for the formation of another pore in this area.

However, two issues were encountered here that prevented from performing experiments with large amount of proteins. The first issue is the measurement set-up (measurement equipment and buffer) which prevents from determining the electroporation threshold for more than 1000 proteins with the employed $1 \mathrm{M} \mathrm{KCl}$ buffer. Only the membrane breakthrough could be studied, and this is much higher and does reflect membrane poration phenomena but would correspond to cell lysis. There, a clear increase could be measured as a function of the protein amount (up to 6000 proteins). This first issue could be solved, for instance, by (i) changing the buffer solution and performing the same experiments again with a buffer with a lower conductivity (100 $\mathrm{mM}$ or $10 \mathrm{mM} \mathrm{KCl})$, by (ii) switching the measurement equipment between the step of protein insertion and the electroporation experiments, or by (iii) inserting a large resistor in parallel between the two electrodes. The first option has been tested here; a lower buffer concentration (100 and 10 $\mathrm{mM} \mathrm{KCl}$ ). However, in that case, the noise level increases as a result of the higher buffer resistance. For the $100 \mathrm{mM}$ buffer, this increase in noise is limited and individual protein insertions can still be distinguished clearly with a 5 pA step in the current trace when $50 \mathrm{mV}$ 
is applied. However, for the $10 \mathrm{mM} \mathrm{KCl}$ buffer, the noise level prevents the detection of individual proteins, and the membranes become very unstable upon protein insertion. These limitations in the dynamic range of the amplifier thus preclude any experiments. The other two options still need to be tested to see if they allow for the insertion of and measurement with more proteins. This issue with the measurement set-up is not found for other studies. For prestin experiments, only a patch of a cell membrane is considered, and this contains lower amount of proteins and the total leakage current is lower. The same applies for gramicidin; the peptide forms pores only transiently so that, most of the time, most of the peptides are not involved in a pore structure. Furthermore, the pore conductance is much lower than for $\alpha$-hemolysin (15 pS) [34]. As a result, the overall the leakage current for gramicidin peptides, even in a much higher concentration, remains lower.

The second issue is found in the first step where proteins insert in the membrane. Obviously, a much higher protein density is required to see an effect of proteins. Although insertions proceed fast with a high concentration of proteins in the solution (see figure 4.5), this is not fast enough and membranes often break before a large amount of proteins has inserted. This indicates that the lifetime and the stability of the membrane may be another limitation. Again, no such limitation is found for other studies. Gramicidin is directly added to the phospholipid mixture employed to prepare BLMs, and no waiting time is involved in the measurements, so membranes with a short lifetime can be employed. Prestin is already expressed and present in a cell or is introduced in vesicles, so the stability of the membranes is not an issue there.

Two other approaches can be conceived to alleviate these two issues; the use of smaller membranes or the use of channel species whose pore is only transiently open. The first solution, a smaller membrane surface area, is easily achieved by miniaturizing the aperture. This would enable such electroporation studies on BLMs containing a relevant density on proteins. Here, the highest density employed for the electroporation experiments is about $1.1 \times 10^{-3}$ proteins $/ \mu \mathrm{m}^{2}$ (1000 proteins) while a natural protein density lies in the $1.25 \times 10^{5}$ proteins $/ \mu \mathrm{m}^{2}$ range.). In other words, our membrane density is 5-6 orders of magnitude too low to perform relevant studies.

Future work will aim at solving this measurement issue to be able to investigate membranes with a higher protein density; this is likely to be achieved by switching equipment between the protein insertion step and the electroporation experiments to meet a greater measurement range. A second approach is to employ smaller membranes made inside a miniaturized or even microfluidic system; they exhibit a higher stability and fewer proteins are needed to reach "interesting" protein densities. Thereby, the influence of $\alpha$ hemolysin on the membrane electroporation can better be investigated.

\section{References}

[1] Ohvo-Rekila, H. et al. (2002). Prog. Lipid Res. 41 (1), pp. 66-97. 
[2] Alberts, B. et al. (1989). Molecular Biology of the Cell. Garland Publishing, Inc: New York, NY, USA

[3] Neumann, E. et al. (1982). EMBO J. 1 (7), pp. 841-845.

[4] Neumann, E. et al., Electroporation and Electrofusion in Cell Biology. Plenum Press: New York, USA, 1989.

[5] Weaver, J.C. \& Chizmadzhev, Y.A. (1996). Bioelectrochem. Bioenerg. 41 (2), pp. 135-160.

[6] Chang, D.C. et al., Guide to Electroporation and Electrofusion. Academic Press, Inc.: San Diego, CA, USA, 1992.

[7] Chen, C. et al. (2006). Medical and Biological Engineering and Computing 44 (1-2), pp. 514.

[8] Golzio, M. et al. (2001). Bioelectrochemistry 53 (1), pp. 25-34.

[9] Chen, C. et al. (2008). Phys. Med. Biol. 53 (17), pp. 4747-4757.

[10] Chernomordik, L.V. et al. (1987). BBA 902 (3), pp. 360-373.

[11] Marrink, S.J. et al. (2009). BBA-Biomembranes 1788 (1), pp. 149-168.

[12] Tieleman, D.P. (2004). Biophys. J. 86 (1), pp. 371a-372a.

[13] Bockmann, R.A. et al. (2008). Biophys. J. 95 (4), pp. 1837-1850.

[14] Hibino, M. et al. (1993). Biophys. J. 64 (6), pp. 1789-1800.

[15] Krassowska, W. \& Filev, P.D. (2007). Biophys. J. 92 (2), pp. 404-417.

[16] Koronkiewicz, S. et al. (2002). BBA-Biomembranes 1561 (2), pp. 222-229.

[17] Melikov, K.C. et al. (2001). Biophys. J. 80 (4), pp. 1829-1836.

[18] Weaver, J.C. (2003). IEEE T. Dielect. El. In. 10 (5), pp. 754-768.

[19] Diederich, A. et al. (1998). Phys. Rev. E 58 (4), pp. 4883-4889.

[20] Ziegler, M.J. \& Vernier, P.T. (2008). J. Phys. Chem. B 112 (43), pp. 13588-13596.

[21] Hui, S.W. \& Sen, A. (1989). Proc. Natl. Acad. Sci. USA 86 (15), pp. 5825-5829.

[22] Chernomordik, L.V. et al. (1985). BBA 812 (3), pp. 643-655.

[23] Kakorin, S. et al. (2005). Biophys. Chem. 117 (2), pp. 155-171.

[24] Koronkiewicz, S. \& Kalinowski, S. (2004). BBA-Biomembranes 1661 (2), pp. 196-203.

[25] Kotulska, M. et al. (2002). Acta Phys. Pol., B 33 (4), pp. 1115-1129.

[26] Needham, D. \& Hochmuth, R.M. (1989). Biophys. J. 55 (5), pp. 1001-1009.

[27] Shil, P. et al. (2008). J. Phys. D: Appl. Phys. 41 (5).

[28] Dekruijff, B. et al. (1976). BBA 436 (4), pp. 729-740.

[29] Israelachvili, J.N. \& Mitchell, D.J. (1975). BBA 389 (1), pp. 13-19.

[30] Karatekin, E. et al. (2003). Biophys. J. 84 (3), pp. 1734-1749.

[31] Chen, Z. \& Rand, R.P. (1997). Biophys. J. 73 (1), pp. 267-276.

[32] Dekker, C.J. et al. (1983). Chem. Phys. Lipids 33 (1), pp. 93-106.

[33] Raffy, S. \& Teissie, J. (1999). Biophys. J. 76 (4), pp. 2072-2080.

[34] Troiano, G.C. et al. (1999). Biophys. J. 76 (6), pp. 3150-3157.

[35] Siu, S.W.I. \& Bockmann, R.A. (2007). Journal of Structural Biology 157 (3), pp. 545-556.

[36] Tarek, M. (2005). Biophys. J. 88 (6), pp. 4045-4053.

[37] Navarrete, E.G. \& Santos-Sacchi, J. (2006). Biophys. J. 90 (3), pp. 967-974.

[38] Troiano, G.C. et al. (1998). Biophys. J. 75 (2), pp. 880-888.

[39] Moldovan, D. et al. (2007). Appl. Phys. Lett. 91 (20).

[40] Murthy, S.N. et al. (2004). J. Controlled Release 98 (2), pp. 307-315.

[41] Tung, L. et al. (1999). Occupational Electrical Injury: An International Symposium 888, 
pp. 249-265.

[42] Gouaux, E. (1998). Journal of Structural Biology 121 (2), pp. 110-122.

[43] Mueller, P. et al. (1963). J. Phys. Chem 67 (2), pp. 534-535.

[44] Wiese, A. \& Seydel, U. (2000). Bacterial toxins: Methods and Protocols. In Methods in Molecular Biology, pp 355-370.

[45] Requena, J. \& Haydon, D.A. (1975). P Roy Soc Lond a Mat 347 (1649), pp. 161-177.

[46] Husslein, T. et al. (1998). J. Chem. Phys. 109 (7), pp. 2826-2832.

[47] Shinoda, W. et al. (2003). J. Phys. Chem. B 107 (50), pp. 14030-14035.

[48] Shinoda, W. et al. (2004). Chem. Phys. Lett. 390 (1-3), pp. 35-40.

[49] Kitano, T. et al. (2003). Chem. Phys. Lipids 126 (2), pp. 225-232.

[50] Cullis, P.R. \& Dekruijff, B. (1979). BBA 559 (4), pp. 399-420.

[51] Papahadjopoulos, D. \& Miller, N. (1967). BBA 135 (4), pp. 624-638.

[52] Rog, T. et al. (2009). BBA-Biomembranes 1788 (1), pp. 97-121.

[53] Demel, R.A. et al. (1967). BBA 135 (1), pp. 11-19.

[54] Bennett, W.F.D. et al. (2009). J. Am. Chem. Soc. 131 (5), pp. 1972-1978.

[55] Keller, S.L. et al. (2000). J. Phys. Chem. B 104 (31), pp. 7522-7527.

[56] Bhakdi, S. \& Tranumjensen, J. (1991). Microbiological Reviews 55 (4), pp. 733-751.

[57] Song, L.Z. et al. (1996). Science 274 (5294), pp. 1859-1866.

[58] Menestrina, G. (1986). J. Membr. Biol. 90 (2), pp. 177-190.

[59] Suzuki, H. \& Takeuchi, S. (2008). Anal. Bioanal. Chem. 391 (8), pp. 2695-2702.

[60] Epand, R.M. (1998). BBA - Rev. Biomemb. 1376 (3), pp. 353-368.

[61] Brown, D.A. \& London, E. (1997). Biochem. Biophys. Res. Commun. 240 (1), pp. 1-7.

[62] Brown, D.A. \& London, E. (2000). J. Biol. Chem. 275 (23), pp. 17221-17224. 


\title{
Chapter 5
}

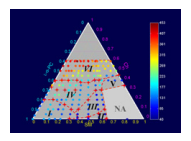

\section{Determination of the electroporation onset of}

\author{
bilayer lipid membranes as a novel approach to
}

establish ternary phase diagrams: example of the

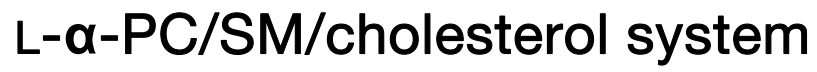

The lipid matrix of cell membranes contains phospholipids belonging to two main classes, glycero- and sphingolipids, as well as cholesterol. This matrix can exist in different phases, liquid disordered $\left(l_{d}\right)$, liquid ordered $\left(l_{0}\right)$ and possibly solid $\left(s_{o}\right)$, or even a combination of these. The precise phase composition of a membrane depends on its molecular content and more specifically on the presence and amount of cholesterol. This in turn dictates the membrane properties. In this work, the resistance of membranes to the process of electroporation is studied and related to the membrane phase composition. Specifically, the threshold voltage for electroporation is measured $\left(V_{t h}\right)$ when DC pulses with increasing amplitude are applied to membranes prepared from various mixtures of a glycerolipid (Heart PC (L- $\alpha$-PC)), a sphingolipid (Egg SM (SM)) and cholesterol (Ch), introduced in various ratios. Binary mixtures (L- $\alpha-\mathrm{PC} / \mathrm{Ch}, \mathrm{L}-\alpha-\mathrm{PC} / \mathrm{SM}, \mathrm{SM} / \mathrm{Ch}$ ) and $\mathrm{L}-\alpha-\mathrm{PC} / \mathrm{SM} / \mathrm{Ch}$ ternary mixtures are successively employed. For all binary and ternary systems, dramatic changes in $V_{\text {th }}$ are measured as a function of the membrane molecular composition, and the variation patterns of $V_{\text {th }}$ are successfully correlated with the membrane phase composition. Interestingly, the measure of the electroporation onset can be employed as a novel methodology to establish ternary phase diagrams, and this is illustrated with the L- $\alpha$ $\mathrm{PC} / \mathrm{SM} /$ cholesterol ternary system.

This chapter has been published in Soft Matter, 6, pp. 4420-4429 (2010). 


\section{Introduction}

One of the main functions of a cell membrane is to act as an impermeable barrier to foreign entities such as genes, drugs or particles. For many applications, like DNA transfection or drug research, transporting foreign entities into a cell is crucial. This is achieved by transiently permeabilizing the cell membrane. A commonly used technique for this purpose is electroporation [1-3] which relies on the application of short pulses (ms and $\mu$ s pulse length) of a high external electric field across a cell [4-5]. When the imposed transmembrane potential reaches a threshold value of about $0.2-1 \mathrm{~V}$, a rearrangement in the molecular structure of the membrane occurs, leading to the creation of (transient) pores and a substantial increase in the cell's permeability. While this technique has been gaining in popularity for the last few decades (especially for cell transfection [6-8]), the overall success rate of the process remains low and still requires further optimization [9].

One approach to increase the electroporation yield consists of getting a better understanding of the mechanism(s) of pore formation and of identifying key-parameters for this process through experimental work and/or theoretical modeling. Experimental studies have demonstrated that pores originate in the lipid matrix of the membrane [10]. Moreover, the time scale for their formation as well as their average size have been determined using both cells [10-12] and model membranes [10, 12-18]. A model for the process of pore formation upon application of a potential across the membrane has been proposed using molecular dynamics simulations. As explained in chapter 2, this process includes three distinct steps [17, 19-20]: (i) a local enhancement of the electric field and the creation of water defects, (ii) the formation of a water file through the membrane to create a hydrophobic pore and (iii) the rearrangement of the phospholipid molecules to create a hydrophilic pore.

Although these theoretical and experimental models have provided insight into the electroporation process, they present a major drawback in that they mostly approximate the membrane as a uniform phospholipid structure $[3,17,21]$, and only a few recent studies use more complex models such as binary phospholipid mixtures [22-23]. By contrast, the actual membrane of a cell is much more complex and this heterogeneity is known to affect its behavior and the resulting electroporation outcome [21, 24]. For instance, Kanduser et al. showed that the cell membrane fluidity is a key-property of membranes for the process of pore formation [24], and this is for a large part regulated by one specific component of cell membranes, cholesterol. The addition of cholesterol to phospholipid membranes changes their stability upon application of an electric field as a result of the specific interactions cholesterol establishes with the phospholipids [21, 25-31]. Interestingly, the effect of cholesterol can be two-fold, depending on both the nature of the phospholipid molecules and the amount of cholesterol. Furthermore, other components of cell membranes, peptides and proteins, also change the membranes' response to the applied electrical field by modifying the membrane properties locally [32-34].

As mentioned, natural membranes are highly complex and they have first been 
described as a two-dimensional fluid with a considerable lateral homogeneity [35-36]. The basic structure and shape of such membranes is defined as a (phospho)lipid matrix which also serves as a substrate for membrane proteins [36]. This fluid mosaic model is dramatically simplifying the membrane structure with respect to the inhomogeneous distribution of the phospholipids and proteins; however, it still forms the basis for more recent and refined models. Phospholipids are amphiphilic molecules that are composed of two main parts: (i) a hydrophilic head consisting of a backbone molecule, a phosphate and a polar group and (ii) two "parallel" hydrophobic chains of various lengths that can contain unsaturations. In natural membranes, two main types of phospholipids are found: glyceroand sphingolipids which have a glycerol and sphingosine backbone, respectively. The length of the hydrophobic chains and the amount of unsaturations together with the nature of the head group and the molecular shape of the phospholipids define the specific molecular properties of these phospholipids. These distinct properties particularly affect their packing pattern and density in membranes and consequently, the membrane fluidity and stability. At their melting temperature $\left(T_{m}\right)$ which is specific for each phospholipid, phospholipids undergo a transition from the gel phase $\left(L_{\beta}\right.$, also called the solid-ordered phase $\left.\left(s_{o}\right)\right)$, where the phospholipids chains are very ordered and the phospholipids can be tilted (indicated with a prime, $\left.L_{\beta}{ }^{\prime}\right)$, to a fluid phase $\left(L_{\alpha}\right.$, also named the liquid disordered phase $\left.\left(l_{d}\right)\right)$ when their chains melt and become disordered [37]. As suggested by the names of the phases, the phospholipids in the latter phase are less ordered than in the former one. This not only means that their diffusion rate in the plane of the membrane is higher but also that their trans/gauche ratio increases [38]. When cholesterol is present in the membranes, phospholipids are also found in a third phase, the liquid ordered phase $\left(l_{0}\right)$ [39]. The $l_{0}$ phase is an intermediate phase between the two other phases; it combines the translational freedom found in the $l_{d}$ phase together with the conformational order specific to the so phase [38, 40-41]. Interestingly, the effect of cholesterol depends on the structural properties of the phospholipids. The two factors that determine the strength of cholesterol interactions with the phospholipids are the presence of unsaturations in the hydrocarbon chains and the nature of the backbone of the phospholipid. Cholesterol prefers saturated phospholipids having a sphingosine backbone [38-39, 42-46]. Consequently, if the three components, glycero-, sphingolipids and cholesterol, are present in a membrane, phase separation occurs due to the fact that cholesterol preferably interacts with sphingolipids. The system undergoes a phase separation into a glycerolipid-rich $l_{d}$ phase and sphingolipid- and cholesterol-rich (micro)domains of $l_{0}$ phase. The size of the microdomain lies between 10 and $200 \mathrm{~nm}$ in cell membranes [47-48] while they have been reported to collide and coalesce in model systems to eventually give large domains [49-50]. In the $l_{0}$ domains, the lateral motion of phospholipid molecules is reduced and the membrane thickness increases due to the chain-straightening effect of cholesterol. $l_{0}$ domains locally act as stiffening spots for the membranes, as is the case for the peptide gramicidin [32, 34]. Consequently, $l_{0}$ domains should contribute to the stabilization of ternary model membranes containing cholesterol. 
In our previous work, the influence of various cell membrane components on the electroporation process was investigated. There, the common synthetic phospholipid 1,2diphytanoyl-sn-glycero-3-phosphocholine (DPhPC) was employed as a membrane basis where (i) other phospholipids and (ii) cholesterol were successively added. The effect of the diversity in the membrane formulation on the resistance to pore formation in these model membranes was measured [31]; this has revealed that (i) the membrane composition is not to be neglected when studying the process of electroporation, and that (ii) the two factors that have the strongest effect on the process of electroporation are the presence of cholesterol and proteins.

Our goal, in this chapter, is to examine further the role of cholesterol, and to better elucidate the mechanisms by which it changes the membrane properties and its resistance to the application of an external electric field. Cholesterol is an essential component of natural membranes. It is a key-element to regulate both the fluidity and the barrier properties of the cell membrane [48, 51-52]. This well-known effect of cholesterol was observed in our previous experiments with a change in the membrane stability in a concentration-dependent manner [31]. However, a simplified system was employed, consisting of a membrane made from only one artificial glycerolipid (DPhPC). DPhPC belongs to the family of phospholipids that is abundantly found in the membranes of archeabacteria, organisms that can resist harsh conditions but whose membranes lack cholesterol. DPhPC molecules form tight networks due to the intertwining of their methylated hydrocarbon chains. DPhPC already condenses the membrane, as would cholesterol do in membranes based on phosphatidylcholines (PCs) which are mostly used for membrane experimentation. Moreover, due to the presence of a mixture of glycerolipids and sphingolipids in natural membranes, cholesterol can give rise to phase separation phenomena [25]. This possible phase separation and its effect on the membrane structure were neglected in our first study as SM was not included. Here, the aim is to reproduce better the complexity found in cell membranes and to study how this would affect the membrane resistance to electroporation. The measured trends in the threshold voltage for electroporation are correlated to (i) the presence of different phases $\left(l_{d}, l_{0}\right.$ or $\left.s_{o}\right)$ as a function of the membrane composition and (ii) the resulting phase separation phenomena (i.e. the co-existence of different phases) induced by cholesterol. For that purpose, ternary systems composed of glycerolipids (Heart PC, a mixture whose predominant species is 16:0/18:2 PC (named as L- $\alpha$-PC in the rest of the chapter)), sphingolipids (Egg SM, a mixture whose predominant species is 16:0 SM (named as $\mathrm{SM}$ in the rest of the chapter)) and cholesterol, in which phase separation phenomena are expected to occur, are utilized. The employed phospholipids are natural mixtures and not purely synthetic phospholipids since the interest of this work lies in studying a system that comes as close as possible to the phospholipid composition found in cell membranes (where over a thousand different phospholipids are found [37]). Our approach consists of looking at the resistance of bilayer lipid membranes (BLMs) to an applied electric field, by increasing stepwise the amplitude of a DC signal applied on the membrane. Firstly, binary systems, L- 
$\alpha$-PC/cholesterol, $\mathrm{L}-\alpha$-PC/SM and $\mathrm{SM} /$ cholesterol are studied to elucidate individual interactions taking place between two molecular species. Following this, ternary systems are investigated to understand how interactions between the three components affect the membrane resistance to electroporation and which parameters are the most important in this. Interestingly, these results enable us to propose a ternary phase diagram for the L- $\alpha$ $\mathrm{PC} / \mathrm{SM} /$ cholesterol system derived from the measured electroporation results of the binary and ternary systems.

\section{Materials and Methods}

\section{A. Chemicals}

Lipids (Heart PC, a mixture whose predominant species is 16:0/ 18:2 PC (L- $\alpha$-PC), Egg $\mathrm{SM}$ a mixture whose predominant species is 16:0 SM (SM), and cholesterol (Ch) (Figure 5.1)) are purchased from Avanti Polar Lipids (Alabaster, AL). $\mathrm{KCl}, \mathrm{n}$-decane and chloroform are purchased from Sigma-Aldrich (St. Louis, MO). Hepes is purchased from Merck Chemicals (Darmstadt, Germany). Deionized water $(18.2 \mathrm{M} \Omega \times \mathrm{cm})$ which is used for all solution preparation and cleaning procedures is obtained using a MilliQ system (Millipore, Billerica, MA).

\section{B. Measurement set-up}

A conventional bilayer system (Warner Instruments, Hamden, CT) is used for BLM experimentation. This system comprises of a Delrin cup and chamber containing two round compartments. The cup is inserted in the trans-compartment of the chamber, and the compartments are connected via a $150 \mu \mathrm{m}$ aperture in the cup across which BLMs are prepared. Both compartments contain $1 \mathrm{~mL}$ buffer solution ( $5 \mathrm{mM}$ Hepes, $1 \mathrm{M} \mathrm{KCl}$, pH 7.4) and $\mathrm{Ag} / \mathrm{AgCl}$ electrodes (used for the electrical characterization of the BLMs). Electrical measurements are carried out with an Axopatch 200b amplifier (Molecular devices, Sunnyvale, CA), applying voltages and measuring currents across the bilayer. Data acquisition is performed with LabVIEW and a PCI-6259 data acquisition card (National Instruments, Austin, TX). All measurements are performed at room temperature.

\section{Preparation and characterization of BLMs}

All lipids are used as chloroform-based solutions. All phospholipids cholesterol are purchased at $10 \mathrm{mg} / \mathrm{mL}$ solutions in chloroform, and cholesterol as a powder and dissolved in chloroform to yield a $25 \mathrm{mg} / \mathrm{mL}$ solution. Before the preparation of BLMs, a few tenths of $\mu \mathrm{L}$ of the lipid solution in chloroform is left to evaporate overnight, yielding an amount of $250 \mu \mathrm{g}$ dried lipids, and subsequently dissolved in n-decane at a final concentration of 25 $\mathrm{mg} / \mathrm{mL}$. To improve the solubility of sphingomyelin and cholesterol $2-3 \%$ ethanol can be added. The resulting n-decane solutions are used for the preparation of BLMs.

Bilayer lipid membranes are created using the Mueller-Rudin technique [53]. Bilayer formation is monitored using the Axopatch amplifier and characterized by a drop in the 
current due to the G $\Omega$ seal between the bilayer and the aperture. Thereafter, the membrane capacitance is measured as previously described to confirm the formation of a BLM [54].

\section{Determination of the electroporation threshold of BLMs}

The electroporation threshold $\left(V_{t h}\right)$ of the membrane is defined as the voltage at which the membrane conductivity increases due to the creation of pore(s). This value is determined as the value at which a leakage current is measured through the membrane. For that purpose, a DC voltage is applied to the membrane using the Axopatch amplifier and gradually increased (every $30 \mathrm{~s}$ ) by steps of $20 \mathrm{mV}$ until peaks larger than three times the root mean square of the noise are observed in the measured current. Pore formation is checked by re-applying the voltage at which peaks are detected; this confirms that the peaks are not due to imperfections in the membrane. The voltage value found corresponds to the electroporation threshold $V_{\text {th }}$. For every membrane composition, $V_{\text {th }}$ is determined at least three independent times and the indicated value corresponds to the average of the three separate measurements.

\section{(Phospho)lipid structures}

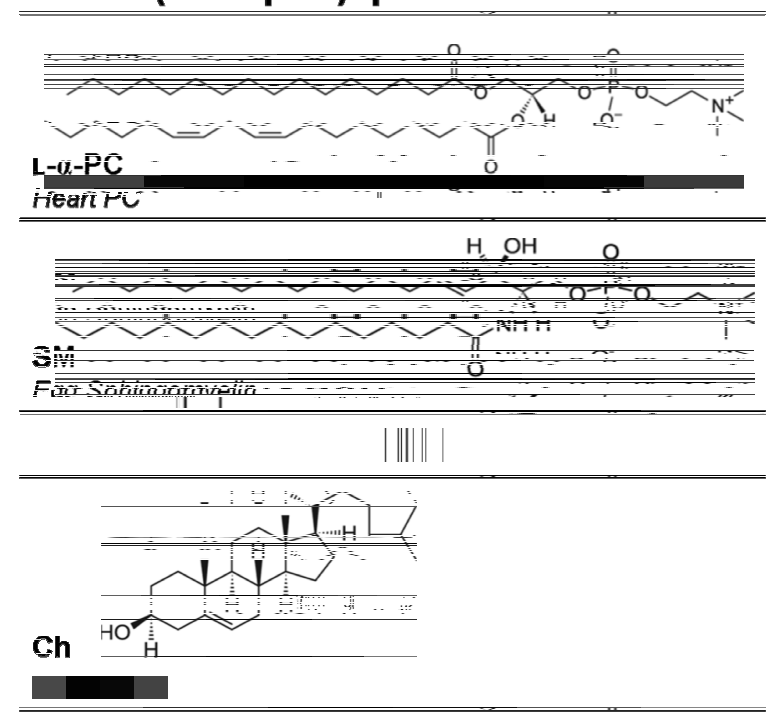

Figure 5.1. Molecular structures of the employed (phospho)lipids

Molecular structures of $L-\alpha$-phosphatidylcholine ( $L-\alpha-P C)$, sphingomyelin (SM), and cholesterol (Ch) used in this study. For $L-\alpha-P C$ and $S M$, the structure of the predominant species found in the mixtures is indicated.

\section{Results and discussion}

The aim of this work is to correlate the resistance of model membranes to the application of an electric field with its composition to get a better understanding of 
processes involved in the technique of electroporation. For that purpose, ternary mixtures of high $T_{m}$ sphingolipids (Egg SM, SM), low $T_{m}$ glycerolipids (heart PC, L- $\alpha$-PC), and cholesterol are employed. These are three key-components of the lipid matrix of cell membranes which are well-known to influence the membrane properties and phase composition. However, in order to be able to comprehend fully the intricate molecular interactions occurring in ternary systems, interactions taking place between different components in the individual binary systems must firstly be characterized.

\section{A. Binary systems}

In the coming section, three different binary systems are considered composed of natural phospholipids: (i) L- $\alpha$-PC/cholesterol, (ii) SM/cholesterol and (iii) L- $\alpha$-PC/SM.

\section{(i) L- $\alpha$-PC/cholesterol}

As shown in figure 5.2, the variations of $V_{\text {th }}$ for L- $\alpha$-PC membranes containing $0-60 \%$ mol cholesterol can be divided in four (concentration-dependent) domains that are indicated by dashed lines in the graph. At low cholesterol concentrations, a progressive decrease in $V_{\text {th }}$ from $165 \pm 19 \mathrm{mV}$ for $0 \%$ cholesterol to $112 \pm 30 \mathrm{mV}$ for $21 \%$ cholesterol is observed. In the next section, $V_{\text {th }}$ rises slowly up to $200 \pm 37 \mathrm{mV}$ upon addition of cholesterol (until $33 \% \mathrm{Ch}$ ). In the third section of the plot, a steeper variation of $V_{\text {th }}$ that reaches the value of $420 \pm 35 \mathrm{mV}$ for $39.5 \%$ cholesterol is seen. Finally, above $39.5 \%$ cholesterol, there is no further change in $V_{\text {th }}$ and a plateau is observed.

Interactions taking place between $\mathrm{L}-\alpha-\mathrm{PC}$ and cholesterol are responsible for these multiple variations in $V_{\text {th }}$ as cholesterol can induce phase transitions in L- $\alpha$-PC/cholesterol mixtures in a concentration-dependent manner. From the literature it is known that a phospholipid having a low $T_{m}$ undergoes one phase transition, notably upon addition of cholesterol, from the $l_{d}$ phase $\left(<\sim 20 \%\right.$ cholesterol) to a pure $l_{0}$ phase at high cholesterol concentrations ( $>\sim 50 \%$ cholesterol). This transition and the phase behavior of a phospholipid depend on its $T_{m}$ [55]. Between these two well-defined phases, an intermediate regime is found where the $l_{d}$ and $l_{0}$ phases coexist [55]. The employed glycerolipid, L- $\alpha$-PC is harvested from natural cell membranes and contains a mixture of different types of glycerolipids so that its transition temperature is hard to pin-point. Nevertheless, its value can be estimated by looking at the transition temperature reported for the glycerolipid that is the most predominant in the mixture. The most abundant species in the mixture is asymmetric with one saturated tail of 16 carbons (16:0) and one double unsaturated tail of 18 carbons (18:2). 16:0/18:2 PCs are reported to have a $T_{m}$ below $-16^{\circ} \mathrm{C}$ [56], so that L- $\alpha$-PC membranes are in the $l_{d}$ phase at room temperature. Although the phospholipids are mobile in this phase, they cannot pack very densely due to kinking of the tails containing unsaturations [51]. Another consequence of the presence of an unsaturated chain is that L- $\alpha$ PC is likely not to interact with cholesterol, as such an interaction is thermodynamically disfavored [25]. Therefore, at low concentrations $(<20 \% \mathrm{Ch})$ there is no positive interaction between cholesterol and L- $\alpha-\mathrm{PC}$, and the amount of defects in the membrane is increased 
due to the negative intrinsic monolayer curvature of cholesterol [43, 57-59]. As the membrane stability directly correlates with the phospholipid packing density, the addition of cholesterol till $20 \%$ facilitates the process of (electro)pore formation. On the contrary, at higher concentrations, cholesterol promotes ordering of the hydrocarbon chains of the phospholipids [25-26, 30]. Furthermore, cholesterol binds the phospholipids together in order to shield itself from the aqueous solution around the membrane, as it does not like to interact with water $[26,60]$. The combination of both the chain ordering and the condensing effects results in a local densification of the phospholipids in the membrane around the molecules of cholesterol. Therefore, the phospholipids that bind to cholesterol are in the $l_{0}$ phase. In that phase, phospholipids are more ordered, packed more tightly and have a relatively high mobility (although lower than in the $l_{d}$ phase) $[38,61]$. In turn, the membrane thickens and better resists the application of an electric field as observed for cholesterol amounts of $20-33 \%$. Nevertheless, this increase in $V_{t h}$ is limited as the fraction of the phospholipids in the $l_{d}$ phase still surpasses that in the $l_{0}$ phase. When the cholesterol amount exceeds $33 \%, V_{\text {th }}$ increases steeply; the fraction of the phospholipids in the $l_{0}$ phase surpasses that of the $l_{d}$ phase, and the strengthening effect of the $l_{0}$ phase is dominant. Furthermore, as the $l_{0}$ domains grow in size but reduce in number due to their coalescence, the amount of packing defects at the interface of the two types of domains becomes smaller, which further disfavors pore formation. This upward trend in $V_{\text {th }}$ would be expected to continue for larger cholesterol contents, but instead $V_{\text {th }}$ levels off above $40 \%$ cholesterol. This plateau is to be accounted for by the maximum solubility of cholesterol in the membrane. The maximum solubility is reported to lie between 50 and $66 \%$, depending of the phospholipid used $[60,62]$. Nevertheless, as cholesterol is reported to dislike interactions with phospholipids containing unsaturated tails such as L- $\alpha$-PC [44], it might already be expelled from the membrane at an even lower concentration than $50 \%$. The measured plateau can reflect this, as the bilayer properties are not changed any further for cholesterol amount above $50 \%$. Membranes prepared with more than $60 \%$ of cholesterol were quickly destabilized, which confirms that cholesterol crystallizes and is expelled out of the membranes.

\section{(ii) $\mathrm{SM} /$ cholesterol}

Following this, SM/cholesterol systems with cholesterol concentrations ranging from 35 to $60 \%$ were studied. Firstly, the transition temperature for natural SM, like the species used in this study, is reported to lie between 37 and $48^{\circ} \mathrm{C}$ [39]. The purely SM membranes are subsequently in the solid phase at room temperature, and SM membranes with a cholesterol amount lower than $35 \%$ cannot be prepared at that temperature. Secondly, as already discussed for L- $\alpha$-PC systems, $60 \%$ cholesterol is the upper limit for cholesterol solubility and no attempt was made to make membranes beyond this cholesterol amount. 


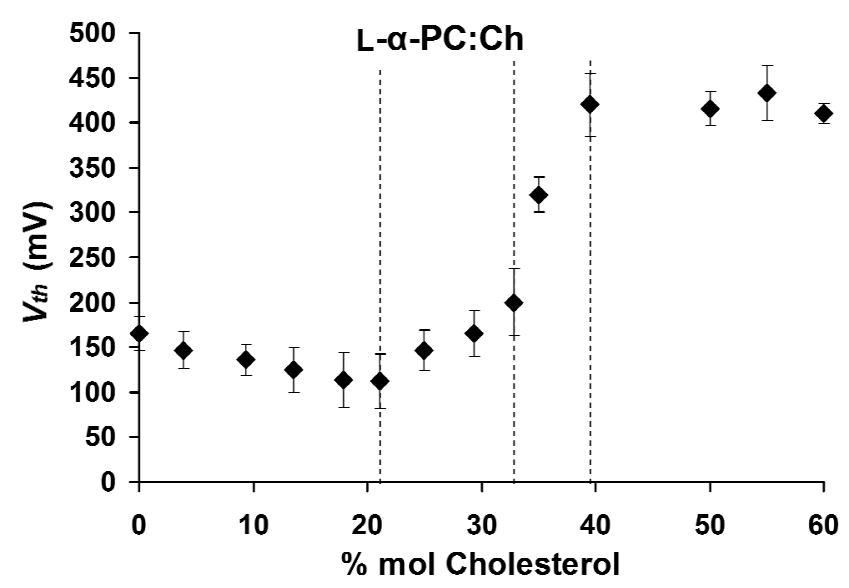

Figure 5.2. Results for the l- $\alpha-P C / C h$ system.

Electroporation threshold $\mathrm{V}_{\text {th }}$ measured for the bi-molecular BLMs containing $L-\alpha-P C$ and cholesterol, plotted as a function of the cholesterol content. Vertical dashed lines depict the transitions between the different domains we distinguish (see text for more information).

As illustrated in figure 5.3, $V_{\text {th }}$ exhibits a two-step increase as a function of the cholesterol content, from $40 \pm 0 \mathrm{mV}$ for membranes containing $35 \%$ cholesterol to $207 \pm 12$ $\mathrm{mV}$ for $45 \%$ cholesterol, and up to $360 \pm 0 \mathrm{mV}$ for $60 \%$ cholesterol. From the literature it is known that SM membranes undergo a transition from the so phase to the $l_{0}$ phase for an increasing cholesterol amount [55]. It is not possible to measure the so phase transition here, as those membranes cannot be prepared. However, their transition to the $l_{o}$ phase is reported for around $25-30 \%$ cholesterol $[38,63]$. Consequently, in the first section of the curve $(35-45 \%$ cholesterol), the membrane contains both $s_{o}$ and $l_{0}$ phases. In the $s_{o}$ phase, the fluidity of the membrane is much lower than in the other phase. The voltage required to electroporate membranes is known to be lower for less fluid membranes [24], and pores do not close easily when lipids move slowly [21]. Furthermore, the structural defects are the largest at the edge between the $s_{o}$ and the $l_{0}$ domains due to the phospholipid mismatch and pore formation should initiate at this interface. The progressive addition of cholesterol to such membranes not only disrupts the dense so phospholipid network and promotes the conversion into the $l_{o}$ phase but also reduces the amount of phospholipid mismatch between the different domains. The membrane becomes more fluid and smoother, and this correlates with a linear increase in the membrane resistance to the applied electric field between 35 and $45 \%$ cholesterol. For a cholesterol amount higher than $45 \%$, the membrane is again reported to be completely in the $l_{0}$ phase [55]. The phospholipids are more organized in the same manner as observed for L- $\alpha$-PC/cholesterol membranes $(>40 \% \mathrm{Ch}$ ). The increase observed in the second section of the plot directly correlates to the transition of the membrane to the $l_{o}$ phase, which is accompanied by its thickening. 


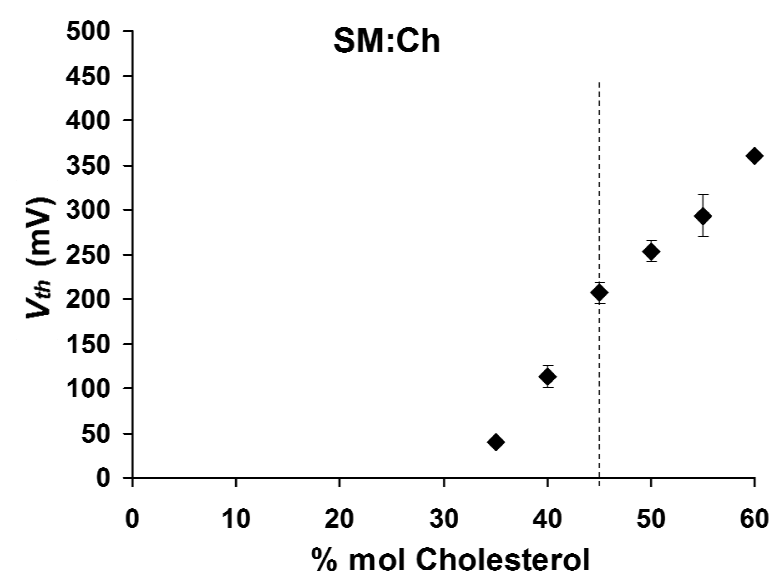

Figure 5.3. Results for the SM/Ch system.

Electroporation threshold $V_{\text {th }}$ measured for the bi-molecular BLMs containing SM and cholesterol, plotted as a function of the cholesterol content. Vertical dashed lines depict the transitions between the different domains we distinguish (see text for more information).

\section{(iii) $\mathrm{L}-\alpha-\mathrm{PC} / \mathrm{SM}$}

Finally, L- $\alpha$-PC/SM binary systems are investigated, and variations in the electroporation threshold for SM amounts from 0 up to $66 \%$ are shown in figure 5.4. As mentioned previously, SM is in the solid phase at room temperature, and this prevents from preparing membranes with more than $66 \% \mathrm{SM}$. A continuous decrease of the electroporation threshold voltage $V_{\text {th }}$ is observed as a function of the addition of SM, with still two different trends. In a first stage, $V_{\text {th }}$ decreases linearly (165 $\pm 19 \mathrm{mV}$ for $0 \% \mathrm{SM}$ to $107 \pm 12 \mathrm{mV}$ for $40 \%$ $\mathrm{SM}$ ) and subsequently levels off up to $66 \% \mathrm{SM}$ (down to $93 \pm 11 \mathrm{mV}$ ). Although the change in the slopes is subtle, this two-fold variation correlates again with two successive phase transitions reported for L- $\alpha$-PC/SM systems (around 30 and $65 \%$ SM, respectively) [55], from the $l_{d}$ phase via the co-existing $l_{d}$ and $s_{o}$ phases, to a pure $s_{o}$ phase. Around $40 \%$ SM, so domains appear in the $l_{d}$ matrix. These domains at first do not destabilize the membrane any further (second zone): the destabilizing properties of enlarging so domains are likely to be counterbalanced by a reduction of the destabilizing L- $\alpha$-PC/SM contacts due to the so domain formation (creation of packing defects). In the last section of the curve (> 66\% SM), the so phase becomes most predominant, preventing stable membrane preparation. 


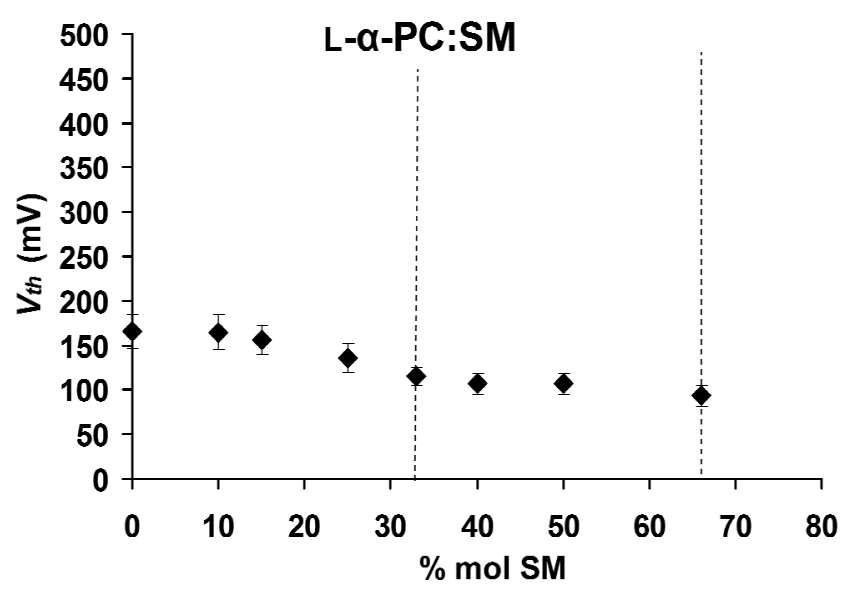

Figure 5.4. Results for the l- $\alpha-P C / S M$ system.

Electroporation threshold $V_{\text {th }}$ measured for the bi-phospholipid BLMs containing L- $\alpha-P C$ and SM, plotted as a function of the SM content. Vertical dashed lines depict the transitions between the different domains we distinguish (see text for more information).

\section{(iv) Ternary system of glycerolipids, sphingolipids and cholesterol}

In the previous section, the individual basic binary interactions occurring between the three lipids, L- $\alpha$-PC, SM and cholesterol, are examined. In the current section, this knowledge is applied to understand interactions found in ternary systems. Not only the precise molecular interactions in such a complex system are of interest, but also how the phase composition of the membrane influences the pore formation process. To study this, cholesterol is added to various binary L- $\alpha$-PC/SM systems, and three examples of the $V_{\text {th }}$ variations measured as a function of the amount of cholesterol are shown in figure 5.5, for ternary systems with a 2:1, a 1:1 and a 1:2 L- $\alpha$-PC/SM molar ratio (Figures 5.5 A, B, C, respectively). All graphs present four regions characterized by different regimes in $V_{\text {th }}$ variations (qualitative boundaries are again represented by dashed lines in the plots), as summarized in Table 5.1. In all three graphs, $V_{\text {th }}$ exhibits a small decrease in the first zone, followed by a rise (second zone) and a plateau (third zone), and finally a second (steep) increase (fourth zone); the precise boundaries for the four zones are indicated in Table 5.1.

As for the binary systems, the different zones in the three graphs correlate to the different phases the phospholipids would be in, according to the literature $[45,55,64]$. When all three components are mixed, the lipids separate in distinct phases, a glycerolipid-rich and cholesterol-depleted $l_{d}$ phase and a sphingolipid-rich and cholesterol-rich $l_{0}$ phase in membranes [25, 38-39, 45, 48, 55, 61, 63-64]. Consequently, both phase transition and phase separation phenomena are expected to occur upon addition of cholesterol in the membranes. Interestingly, the different regions in our graphs (See figure 5.5 and Table 5.1) coincide with the phase domains reported for similar systems [45, 55, 64]. Table 5.1 
summarizes the trends found in $V_{\text {th }}$ together with the phenomena observed in the membrane in terms of phase composition and structure changes. Briefly, for all three systems, a progressive transition is observed from a $l_{d}\left(2: 1 \mathrm{~L}-\alpha-\mathrm{PC} / \mathrm{SM}\right.$ system) or a mixed $l_{d}-s_{o}$ phase (1:1 and 1:2 L- $\alpha$-PC/SM systems) into a full $l_{0}$ membrane [55], with intermediary states with a coexisting (i) $l_{d}$ and $l_{0}$ phases (2:1 L- $\alpha$-PC/SM system) or (ii) $l_{d}, l_{0}$, and $s_{o}(1: 1$ and 1:2 L- $\alpha$ $\mathrm{PC} / \mathrm{SM}$ systems). The variations in the different zones are detailed below.

\section{Zone 1 (ld and $l_{\mathrm{d}}-$ so phase)}

In this first zone, $V_{\text {th }}$ progressively decreases for all three systems; the higher the amount of SM, the steeper the decrease. However, this variation is not accounted for by a phase transition but is linked to the precise molecular interactions in the membrane upon addition of cholesterol. The behavior of the first system is comparable to a membrane prepared from L- $\alpha$-PC only (See figure 5.2): cholesterol is inserted between the phospholipids but does not develop any positive interactions with them. As before, the packing density of the phospholipid molecules is reduced, and pore formation is facilitated. For the two other systems, the situation is more complex as SM is in very tightly packed so domains, and most probably tilted [65]. Consequently, cholesterol accommodates itself first into $l_{d}$ L- $\alpha$-PC domains, as for the former system. However, part of the cholesterol still interacts with the so domains where SM molecules become untilted while remaining in the so phase. As before, the phospholipids are less densely packed, as reflected by the reduction measured in $V_{\text {th. }}$ Interestingly, as the content in SM in the membrane is greater, the decline in $V_{\text {th }}$ is more marked. This is related to the presence of more and larger so domains: not only the membrane becomes stiffer [24], but the amount of packing defects between the domains is higher.

As a conclusion, for the three systems, the addition of a limited amount of cholesterol translates into the creation of packing defects in the membrane, which is in turn less resistant to the application of an electric field.

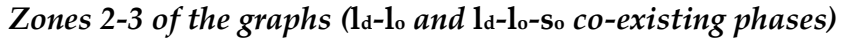

In the two following zones (zones 2-3), $V_{\text {th }}$ displays a two-step variation upon increasing cholesterol content: it first rises (zone 2) and subsequently gives a plateau (zone 3). As before, although the trends are similar for all three graphs, the precise phenomena responsible for these variations differ for the three graphs, as detailed in Table 5.1. The addition of cholesterol in membranes that are initially fully in the $l_{d}$ phase (2:1 L- $\alpha$-PC:SM ratio) triggers the successive phase transition of SM (zone 2) and PC (zones 2-3) into the $l_{0}$ phase [55]. From the binary mixtures (see figure 5.3) we know that SM/Ch membranes are fully $l_{o}$ when the SM:chol ratio reaches 11:9. At the border between zones 2 and 3, the composition of the membrane is $23 \% \mathrm{SM}, 47 \% \mathrm{~L}-\alpha-\mathrm{PC}$ and $30 \% \mathrm{Ch}$; the transformation of SM into $l_{0}$ domains costs $20.5 \%$ of the $30 \%$ cholesterol. The remaining amount of cholesterol is subsequently employed to partially convert L- $\alpha$-PC into the $l_{o}$ phase. Subsequently, only in the third zone of the graph, $l_{d} \mathrm{~L}-\alpha$-PC domains completely disappear into L- $\alpha$-PC $l_{o}$ domains. 

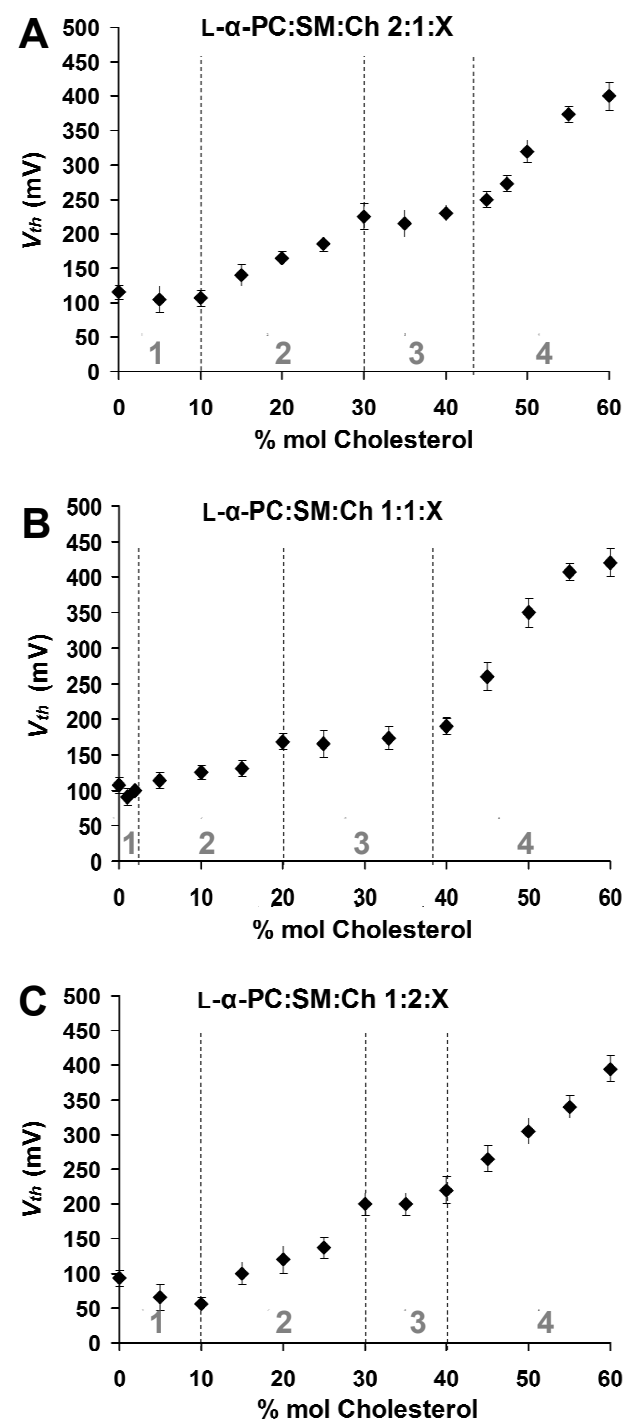

Figure 5.5. Results for three ternary (L- $\alpha-P C / S M / C h)$ systems.

Electroporation threshold $\mathrm{V}_{\text {th }}$ measured for BLMs made from a ternary mix of (A) 2:1 L- $\alpha$-PC:SM, (B) 1:1 L- $\alpha$ $P C: S M$ and (C) 1:2 L- $\alpha-P C: S M$ with increasing amounts of cholesterol, plotted as a function of the cholesterol content. Vertical dashed lines depict the transitions between the different domains we distinguish and the gray numbers in each domain refers to the different zones (see text for more information).

However, $V_{\text {th }}$ exhibits a plateau in the zone 3 , and not an increase as would be expected from the full conversion of the membrane into the $l_{o}$ phase, revealing the existence of a 
counterbalancing effect. The growth of $\mathrm{L}-\alpha-\mathrm{PC} l_{0}$ domains is accompanied by the creation of more packing defects at the border between L- $\alpha$-PC and SM $l_{0}$ domains due to an increase in line tension and a difference in both the packing density $[48,50,65]$ and domains thickness. This effect is negligible in the second zone as L- $\alpha-\mathrm{PC}$ is distributed between the $l_{d}$ and $l_{0}$ phases and the amount of L- $\alpha$-PC $l_{0}$ domains remains small. The creation of additional packing defects is the counteracting force, accounting for the plateau found in $V_{t h}$.

The 1:1 and 1:2 L- $\alpha$-PC/SM ratio membranes are in an intermixed $l_{d}$ - so phase at low cholesterol concentrations (5 and 10\% respectively). Upon addition of cholesterol SM is again first converted into $l_{0}$ domains, and the three phases co-exist (zone 2 in the graphs). The transformation of the SM so domains into $l_{0}$ domains thickens the membrane and strengthens its resistance against pore formation, as reflected by the increase in $V_{t h}$. In this zone, L- $\alpha$-PC molecules are unaffected and remain in the $l_{d}$ phase. For higher cholesterol contents (border between zone 2 and zone 3), all so domains have disappeared and the membrane is found in an intermixed $l_{d}-l_{0}$ phase. This $l_{d}-l_{0}$ region exhibits the same behavior as the 2:1 L- $\alpha$-PC/SM systems, and the two same counteracting effects are found, giving rise to a plateau of $V_{\text {th }}$.

\section{Zone 4 (lo phase)}

For the highest cholesterol contents (fourth zone), all membranes are in a pure $l_{o}$ phase $[45,55,64]$; they are consequently the most resistant to pore formation. As SM and L- $\alpha$-PC give different packing pattern in the $l_{0}$ phase, they form separate domains. The large $V_{t h}$ values in this last zone correlate to the denser phospholipid packing in both $l_{0}$ domains upon further addition of cholesterol. Interestingly, the maximum value measured for $V_{\text {th }}$ for $l_{0}$ membranes phase is slightly lower for membranes containing higher amounts of SM. SM $l_{0}$ domains are thinner than their counterparts formed from L- $\alpha$-PC. Consequently, the average thickness of the membrane decreases for larger contents in SM, and this is directly reflected in the behavior of $V_{t h}$.

\section{Proposed L- $\alpha-P C / S M /$ cholesterol ternary phase diagram}

As the trends found in the variations of $V_{\text {th }}$ could be correlated with the phase composition of the membrane, and as they were in good agreement with other data reported in the literature [55], the same measurements were subsequently carried out for other L- $\alpha$ $\mathrm{PC} / \mathrm{SM} /$ cholesterol ternary mixtures, in an attempt to derive a full ternary phase diagram for this L- $\alpha$-PC/SM/cholesterol system. Figure 5.6 depicts the resulting ternary diagram. First, the region at the top $(>60 \% \mathrm{Ch})$ and the striped region at the right bottom corner of the graph $(>66 \% \mathrm{SM}$ and $<40 \% \mathrm{Ch}$ ) correspond, respectively, to the region that is unavailable for measurements due to the large amount of cholesterol, and to the region where the so phase dominates, and this prevents the preparation of stable membranes, as mentioned earlier. For every region, the membrane phase composition can successfully be identified from the $V_{\text {th }}$ variation pattern as before, and the resulting full diagram reasonably correlates to other ternary phase diagrams $[45,55,64,66]$. However, one region in the diagram still 
Table 5.1. Summary of the observed phenomena for the different zones.

Summary of the phenomena observed for the BLM electroporation experiments for the three l- $\alpha-P C / S M / C h$ ternary systems having a 2:1, 1:1 and 1:2 L- $\alpha$-PC:SM molar ratio and presented in figure 5.5: borders of the 4 zones where the variation of $V_{\text {th }}$ is monotonous, sense of $V_{\text {th }}$ variations, phase composition of the membrane and major changes occurring in both the phase composition and the structure of the membrane.

\begin{tabular}{|c|c|c|c|c|}
\hline \multicolumn{2}{|r|}{ Zone (details) } & 2:1 PC/SM & 1:1 PC/SM & 1:2 PC/SM \\
\hline \multirow{4}{*}{1} & Borders & $0 \rightarrow 10 \% \mathrm{Ch}$ & $0 \rightarrow 2 \% \mathrm{Ch}$ & $0 \rightarrow 10 \% \mathrm{Ch}$ \\
\hline & $V_{\text {th }}$ variation & $\sqrt{3}$ & $\sqrt{3}$ & ת \\
\hline & $\begin{array}{l}\text { Phase } \\
\text { composition }\end{array}$ & $l_{d}(\mathrm{SM} ; \mathrm{L}-\alpha-\mathrm{PC})$ & \multicolumn{2}{|c|}{$\begin{array}{c}s_{o}(\mathrm{SM}) \\
l_{d}(\mathrm{~L}-\alpha-\mathrm{PC})\end{array}$} \\
\hline & $\begin{array}{l}\text { Changes } \\
\text { (Phase; } \\
\text { Structure) }\end{array}$ & \multicolumn{3}{|c|}{ Packing defect creation ${ }^{b}$} \\
\hline \multirow[b]{4}{*}{2} & Borders & $10 \rightarrow 30 \% \mathrm{Ch}$ & $2 \rightarrow 20 \% \mathrm{Ch}$ & $10 \rightarrow 30 \% \mathrm{Ch}$ \\
\hline & $V_{t h}$ variation & 仓े & $\hat{v}$ & $\hat{\imath}$ \\
\hline & $\begin{array}{l}\text { Phase } \\
\text { composition }\end{array}$ & $l_{d}+l_{o}(\mathrm{SM} ; \mathrm{L}-\alpha-\mathrm{PC})$ & \multicolumn{2}{|c|}{$\begin{array}{c}s_{o}+l_{o}(\mathrm{SM}) \\
l_{d}+l_{0}(\mathrm{~L}-\alpha-\mathrm{PC})\end{array}$} \\
\hline & $\begin{array}{l}\text { Changes } \\
\text { (Phase; } \\
\text { Structure) }\end{array}$ & $\begin{array}{c}\text { SM: } l_{d} \rightarrow l_{o} \\
\text { L- } \alpha-\mathrm{PC}: l_{d} \rightarrow l_{0} \\
\text { 仓 Thickness }{ }^{c} \\
\text { 仓 Packing density }{ }^{c}\end{array}$ & $\begin{array}{c}\text { SM: } s_{o} \rightarrow l_{o} \\
\left(\mathrm{~L}-\alpha-\mathrm{PC}: l_{d} \rightarrow l_{0}\right) \\
\text { 仓े Thickness }{ }^{c} \\
\text { 仓े Packing density }{ }^{c}\end{array}$ & $\begin{array}{c}\text { SM: } s_{o} \rightarrow l_{0} \\
\text { 仓 Thickness }{ }^{c} \\
\text { 仓 Packing density }{ }^{c}\end{array}$ \\
\hline \multirow{4}{*}{3} & Borders & $30 \rightarrow 42 \% \mathrm{Ch}$ & $20 \rightarrow 38 \% \mathrm{Ch}$ & $30 \rightarrow 42 \% \mathrm{Ch}$ \\
\hline & $V_{\text {th }}$ variation & Plateau & Plateau & Plateau \\
\hline & $\begin{array}{c}\text { Phase } \\
\text { composition }\end{array}$ & \multicolumn{3}{|c|}{$\begin{array}{c}l_{0}(\mathrm{SM}) \\
l_{d}+l_{0}(\mathrm{~L}-\alpha-\mathrm{PC})\end{array}$} \\
\hline & $\begin{array}{l}\text { Changes } \\
\text { (Phase; } \\
\text { Structure) }\end{array}$ & \multicolumn{3}{|c|}{$\begin{array}{c}\text { 仓 Thickness }{ }^{c} \\
\text { 仓 Packing density }{ }^{c} \text { Packing defect creation }{ }^{b}\end{array}$} \\
\hline \multirow{4}{*}{4} & Borders & $42 \rightarrow 60 \% \mathrm{Ch}$ & $38 \rightarrow 60 \% \mathrm{Ch}$ & $42 \rightarrow 60 \% \mathrm{Ch}$ \\
\hline & $V_{\text {th }}$ variation & 仓ิ & 仓ิ & 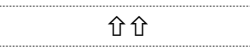 \\
\hline & $\begin{array}{c}\text { Phase } \\
\text { composition }\end{array}$ & \multicolumn{3}{|c|}{$l_{o}(\mathrm{SM} ; \mathrm{L}-\alpha-\mathrm{PC})$} \\
\hline & $\begin{array}{l}\text { Changes } \\
\text { (Phase; } \\
\text { Structure) }\end{array}$ & \multicolumn{3}{|c|}{$\begin{array}{l}\quad-a \\
\text { 仓 Thickness }{ }^{c} \\
\text { 仓 Packing density }{ }^{c}\end{array}$} \\
\hline
\end{tabular}

remains elusive; it corresponds to the so-called "three-phase-triangle", where all three phases $\left(l_{o}, l_{d}, s_{0}\right)$ co-exist $[50,55]$. In this region, $V_{\text {th }}$ remains relatively stable between 60 and $130 \mathrm{mV}$, compared to the variations observed in pure $l_{d}$ and intermixed $l_{d}-s_{o}$ domains. As 
discussed already, this is caused by two counteracting effects: the growth of $l_{0}$ domains and the apparition of additional packing defects due to a phospholipid mismatch at the domain border.

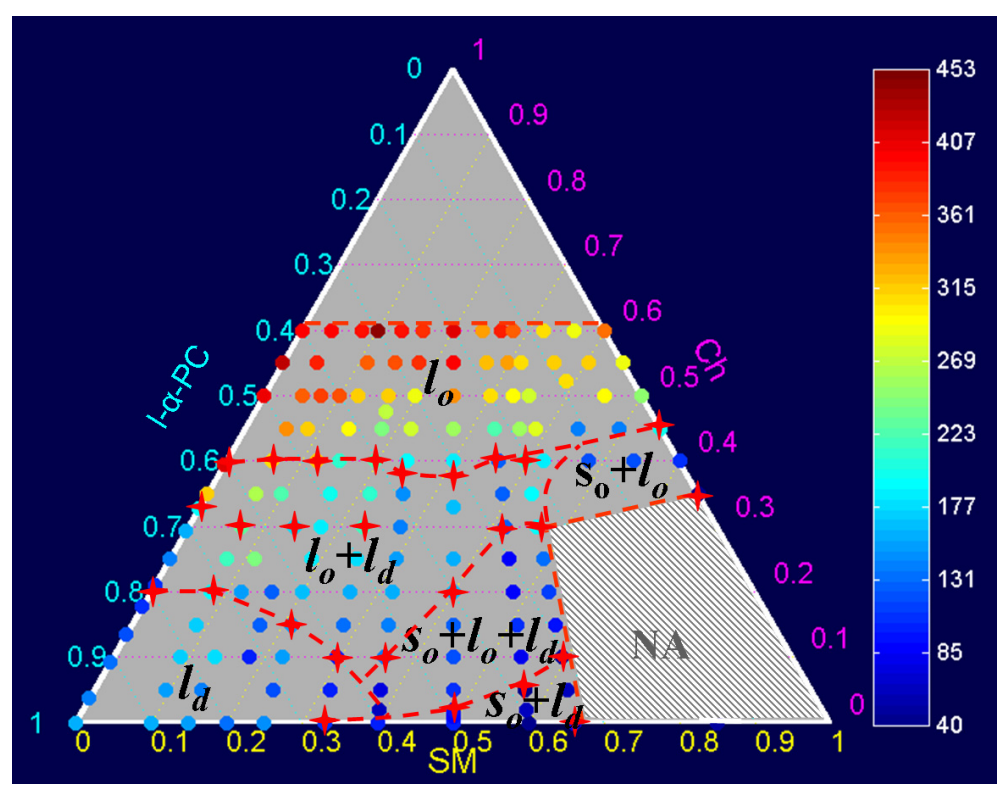

Figure 5.6. Ternary phase diagram.

Ternary graph derived using a novel technique: determining the membranes resistance to electroporation. The dots indicate individual values of electroporation threshold $\mathrm{V}_{\text {th }}$ in $\mathrm{mV}$ for various ternary mixtures made from $\mathrm{l}$ $\alpha-P C, S M$ and cholesterol. The red stars indicate the phase transition boundaries for the discussed mixture and dashed lines possible phase boundaries.

The full diagram clearly shows that the membrane composition strongly influences $V_{t h}$, and the steepest variations are found for high cholesterol concentrations (> 40\%) where small changes in the membrane composition translate into dramatic alterations in $V_{\text {th }}$. Interestingly, the composition of natural cell membranes is found in this area: this indicates that small changes in the lipidic composition of a cell membrane would have a large effect on its response to the application of an electric field and on the outcome of the electroporation process. Moreover, the plasma membrane composition not only differs per cell type, but also per individual cell in the same culture or even locally in the cell membrane. As a consequence, each cell, and even different parts of the membrane, would respond differently to an applied electric field, explaining why the outcome of electroporation experiments varies so much for every cell. Consequently, the size of the cell is not the only factor to be taken into account when designing an electroporation protocol.

\section{Differences between our diagram and previously reported diagrams}

The precise phase boundaries defined by the variation trends of $V_{\text {th }}$ do not fully 
correspond to those reported in the literature [45, 55, 64]. A first reason for this is that the PC species used here is different from those employed in those other studies (palmitoyloleoylphosphatidylcholine (POPC) [45, 55, 64] or dioleoylphosphatidylcholine (DOPC) [45]. Secondly, L- $\alpha$-PC and SM employed here are obtained from natural cell membranes: consequently, they are mixtures of several species, and not pure phospholipids as is the case in the other reports, and they contain impurities. The latter are known to have a dramatic effect on the overall behavior of the membrane [50]. On other aspects, the phase diagrams described in the literature have been obtained using a combination of other techniques, such as nuclear magnetic resonance (NMR) [67-69], electron spin resonance (ESR) [70], fluorescence microscopy [45, 55, 68, 71], differential scanning calorimetry (DSC) [64] and atomic force microscopy (AFM) [72-74] while here only electrical measurements are utilized. The technique employed to establish the diagram is also of influence on the precisely found borders between the regions in the diagram. Moreover, some of the aforementioned techniques require the addition of probes or the use of modified phospholipids, and this is notably the case for fluorescence measurements. Obviously, the presence of these probes affects the phospholipid packing density and changes the membrane phase behavior [45]. All these factors account for any difference found between these data and other data reported in literature.

\section{Conclusion}

\section{A. Electroporation threshold for complex membrane mixtures}

Most electroporation studies reported in the literature have been performed with only one type of phospholipid in the membrane, neglecting thereby the diversity found in natural cell membranes. Therefore, membranes combining natural glycero- and sphingolipids as well as cholesterol are employed here. Such models better represent what is happening in natural membranes when their composition is varied (either artificially, or between cells, or by the cell itself).

These model membranes give great variations in the threshold voltage for electroporation as a function of the membrane composition, which supports our approach. Interestingly, in the region where a cell membrane would fall in, slight changes in the composition have a large effect on its stability and the outcome of the electroporation process. This finding is highly relevant as the membrane composition of all cells differs, but still within a limited window. Even in a single cell, the composition of the membrane is not homogenous, especially when the cell is polarized with highly different membrane properties in the different parts. For instance, the apical part of epithelial cells contains much more sphingolipids and cholesterol compared to their basolateral part [38, 61]. A direct consequence, according to the results of this chapter, is that the basolateral part of the membrane would be electroporated with milder conditions (lower voltage). In other words, slight changes in the lipidic membrane composition and the cell orientation must be taken into account for the electroporation protocol. This is currently under investigation in our lab, 
by using either model membranes whose composition is derived from that of the different parts of polarized cells or epithelial cells (MDCK cells). These properties of a cell explain why electroporation experiments are hard to control, also at the single cell level.

Nevertheless, advantage can also be taken from this effect, by controlling the orientation of the cell during the electroporation process so that the basolateral part and not the apical part of the membrane is porated; thereby, the success rate might be enhanced. Similarly, cancer cells are known to have an altered membrane composition compared to their healthy counterparts. The membranes of malignant cells often contain different amounts of cholesterol, sphingolipids and glycerolipids [75]. A direct consequence is that cancer cells would be electroporated at a different voltage than healthy cells. This knowledge can be used to design specific in vivo protocols to treat cancer cells while leaving healthy cells untouched (or vice-versa).

\section{B. Electroporation measurements as a novel approach for establishing ternary diagrams}

The determination of the membrane response to the application of an electric field is shown here as an alternative technique to establish quickly a phase diagram for ternary lipid mixtures. This technique does not require any additional probe or sophisticated equipment, and it is straightforward to record the lipid phase composition. However, electroporation measurements only provide second order information about the membrane phases, as it is not possible to literally "see" the changes in the membrane, its structure as well as its phase composition. Although a good correlation is shown here between the electroporation results and phase transition boundaries reported in literature, it would still be interesting to couple such electrical measurements to more conventional techniques or to investigate a system already described in the literature using our novel methodology. On other aspects, using microscopy techniques, nanodomains cannot be visualized, whereas they are detectable using spectroscopy techniques [76]. Similarly, it would be interesting to determine the sensitivity of the presently described methodology.

Ternary phase diagrams are currently determined using a combination of various techniques. This combined approach is labor-intensive and time-consuming, requiring several years of study before a diagram is fully determined. Interestingly, the approach proposed here lends itself well to multiplexing, especially when combined with microfluidics in lab-on-a-chip devices [77-79], a field in which our group has much experience [80-81]. By using integrated microfluidic systems containing a series of independently addressed BLMs, the determination of the threshold voltage could be performed on different mixtures simultaneously and the phase behavior could be determined both optically and electrically. Such a microfluidic device would consist of a quick screening tool for the phase domains, after which the interesting regions could be explored in more detail. Furthermore, the time and manual labor could be further reduced by using automated membrane preparation and experimentation techniques [78-79]. These 
microfluidic devices are already under development, but their interest has till now been limited to pharmaceutical applications for high-throughput screening assays of drugs on membrane proteins [78-79].

Although complex systems were studied in this paper, these remain simplified models of the cell membrane whose lipid matrix contains over 100 different types of molecules. Besides this, $50 \%$ weight of the membranes corresponds to proteins. We have shown here that mixtures of PC, SM and cholesterol give better models of cell membranes. However, two other main components of a cell membrane: glycolipids and proteins, are still missing. In further studies these should be included and their effect investigated.

\section{Acknowledgments}

Professor Manuel Prieto from the Centro Química-Física Molecular (Lisbon, Portugal) for is thanked for critically reading the manuscript this chapter is based on and for his useful comments.

\section{References}

[1] Neumann, E. et al. (1982). EMBO J. 1 (7), pp. 841-845.

[2] Neumann, E. et al., Electroporation and Electrofusion in Cell Biology. Plenum Press: New York, USA, 1989.

[3] Weaver, J.C. \& Chizmadzhev, Y.A. (1996). Bioelectrochem. Bioenerg. 41 (2), pp. 135-160.

[4] Chang, D.C. et al., Guide to Electroporation and Electrofusion. Academic Press, Inc.: San Diego, CA, USA, 1992.

[5] Fox, M.B. et al. (2006). Anal. Bioanal. Chem. 385 (3), pp. 474-485.

[6] Chen, C. et al. (2006). Med. Biol. Eng. Comput. 44 (1-2), pp. 5-14.

[7] Golzio, M. et al. (2001). Bioelectrochemistry 53 (1), pp. 25-34.

[8] Valero, A. et al. (2008). Lab Chip 8 (1), pp. 62-67.

[9] Chen, C. et al. (2008). Phys. Med. Biol. 53 (17), pp. 4747-4757.

[10] Chernomordik, L.V. et al. (1987). BBA 902 (3), pp. 360-373.

[11] Hibino, M. et al. (1993). Biophys. J. 64 (6), pp. 1789-1800.

[12] Weaver, J.C. (2003). IEEE T. Dielect. El. In. 10 (5), pp. 754-768.

[13] Bockmann, R.A. et al. (2008). Biophys. J. 95 (4), pp. 1837-1850.

[14] Koronkiewicz, S. et al. (2002). BBA-Biomembranes 1561 (2), pp. 222-229.

[15] Krassowska, W. \& Filev, P.D. (2007). Biophys. J. 92 (2), pp. 404-417.

[16] Melikov, K.C. et al. (2001). Biophys. J. 80 (4), pp. 1829-1836.

[17] Tieleman, D.P. (2004). BMC Biochem 5.

[18] Winterhalter, M. (1999). Colloid. Surface. A 149 (1-3), pp. 161-169.

[19] Marrink, S.J. et al. (2009). BBA-Biomembranes 1788 (1), pp. 149-168.

[20] Ziegler, M.J. \& Vernier, P.T. (2008). J. Phys. Chem. B 112 (43), pp. 13588-13596.

[21] Shil, P. et al. (2008). J. Phys. D: Appl. Phys. 41 (5).

[22] Vernier, P.T. et al. (2009). Plos One 4 (11).

[23] Vernier, P.T. et al. (2006). J. Am. Chem. Soc. 128 (19), pp. 6288-6289.

[24] Kanduser, M. et al. (2006). Eur. Biophys. J Biophys. 35 (3), pp. 196-204.

[25] Almeida, P.F.F. (2009). BBA-Biomembranes 1788 (1), pp. 72-85. 
[26] Berkowitz, M.L. (2009). BBA-Biomembranes 1788 (1), pp. 86-96.

[27] Kakorin, S. et al. (2005). Biophys. Chem. 117 (2), pp. 155-171.

[28] Koronkiewicz, S. \& Kalinowski, S. (2004). BBA-Biomembranes 1661 (2), pp. 196-203.

[29] Kotulska, M. et al. (2002). Acta Phys. Pol., B 33 (4), pp. 1115-1129.

[30] Rog, T. et al. (2009). BBA-Biomembranes 1788 (1), pp. 97-121.

[31] van Uitert, I. et al. (2010). BBA - Biomembranes 1798 (1), pp. 21-31.

[32] Siu, S.W.I. \& Bockmann, R.A. (2007). J. .Struct. Biol. 157 (3), pp. 545-556.

[33] Tarek, M. (2005). Biophys. J. 88 (6), pp. 4045-4053.

[34] Troiano, G.C. et al. (1999). Biophys. J. 76 (6), pp. 3150-3157.

[35] Jacobson, K. et al. (1995). Science 268 (5216), pp. 1441-1442.

[36] Singer, S.J. \& Nicolson, G.L. (1972). Science 175 (4023), pp. 720-731.

[37] Heimburg, T. (2007). Thermal Biophysics of Membranes. Wiley-VCH Verlag GmbH: Wienheim, Germany

[38] Simons, K. \& Vaz, W.L.C. (2004). Annu. Rev. Biophys. Biomol. Struct. 33, pp. 269-295.

[39] Ramstedt, B. \& Slotte, J.P. (2006). BBA-Biomembranes 1758 (12), pp. 1945-1956.

[40] Brown, D.A. \& London, E. (2000). J. Biol. Chem. 275 (23), pp. 17221-17224.

[41] Silvius, J.R. (2003). BBA-Biomembranes 1610 (2), pp. 174-183.

[42] Cullis, P.R. \& Dekruijff, B. (1979). BBA 559 (4), pp. 399-420.

[43] Israelachvili, J.N. \& Mitchell, D.J. (1975). BBA 389 (1), pp. 13-19.

[44] Smaby, J.M. et al. (1994). Biochemistry 33 (31), pp. 9135-9142.

[45] Veatch, S.L. \& Keller, S.L. (2005). Phys. Rev. Lett. 94 (14).

[46] Wang, T.Y. \& Silvius, J.R. (2000). Biophys. J. 79 (3), pp. 1478-1489.

[47] Pike, L.J. (2006). J. Lipid Res. 47 (7), pp. 1597-1598.

[48] Semrau, S. \& Schmidt, T. (2009). Soft Matter 5 (17), pp. 3174-3186.

[49] Collins, M.D. \& Keller, S.L. (2008). Proc. Natl. Acad. Sci. USA 105 (1), pp. 124-128.

[50] Veatch, S.L. \& Keller, S.L. (2003). Biophys. J. 85 (5), pp. 3074-3083.

[51] Alberts, B. et al. (1989). Molecular Biology of the Cell. Garland Publishing, Inc: New York, NY, USA

[52] Ohvo-Rekila, H. et al. (2002). Prog. Lipid Res. 41 (1), pp. 66-97.

[53] Mueller, P. et al. (1963). J. Phys. Chem 67 (2), pp. 534-535.

[54] Wiese, A. \& Seydel, U. (2000). In Methods in Molecular Biology, pp 355-370.

[55] de Almeida, R.F.M. et al. (2003). Biophys. J. 85 (4), pp. 2406-2416.

[56] Lipid Thermotropic Phase Transition Database, http://www.lipidat.ul.ie

[57] Dekruijff, B. et al. (1976). BBA 436 (4), pp. 729-740.

[58] Hui, S.W. \& Sen, A. (1989). Proc. Natl. Acad. Sci. USA 86 (15), pp. 5825-5829.

[59] Karatekin, E. et al. (2003). Biophys. J. 84 (3), pp. 1734-1749.

[60] Huang, J.Y. \& Feigenson, G.W. (1999). Biophys. J. 76 (1), pp. A272-A272.

[61] Marsh, D. (2009). BBA-Biomembranes 1788 (10), pp. 2114-2123.

[62] Huang, J.Y. et al. (1999). BBA-Biomembranes 1417 (1), pp. 89-100.

[63] Kahya, N. et al. (2003). J. Biol. Chem. 278 (30), pp. 28109-28115.

[64] Pokorny, A. et al. (2006). Biophys. J. 91 (6), pp. 2184-2197.

[65] Feigenson, G.W. (2006). Nat. Chem. Biol. 2 (11), pp. 560-563.

[66] Feigenson, G.W. (2009). BBA-Biomembranes 1788 (1), pp. 47-52.

[67] Thewalt, J.L. \& Bloom, M. (1992). Biophys. J. 63 (4), pp. 1176-1181. 
[68] Veatch, S.L. et al. (2004). Biophys. J. 86 (5), pp. 2910-2922.

[69] Vist, M.R. \& Davis, J.H. (1990). Biochemistry 29 (2), pp. 451-464.

[70] Collado, M.I. et al. (2005). Biochemistry 44 (12), pp. 4911-4918.

[71] Feigenson, G.W. \& Buboltz, J.T. (2001). Biophys. J. 80 (6), pp. 2775-2788.

[72] Milhiet, P.E. et al. (2001). Biophys. J. 81 (1), pp. 547-555.

[73] Milhiet, P.E. et al. (2002). J. Biol. Chem. 277 (2), pp. 875-878.

[74] Yuan, C.B. \& Johnston, L.J. (2001). Biophys. J. 81 (2), pp. 1059-1069.

[75] Hendrich, A.B. \& Michalak, K. (2003). Curr. Drug Targets 4 (1), pp. 23-30.

[76] Goni, F.M. et al. (2008). Biochim Biophys Acta 1781 (11-12), pp. 665-84.

[77] Le Pioufle, B. et al. (2008). Anal. Chem. 80 (1), pp. 328-332.

[78] Suzuki, H. et al. (2009). Biomed. Microdevices 11 (1), pp. 17-22.

[79] Zagnoni, M. et al. (2009). Biosens. Bioelectron. 24 (5), pp. 1235-1240.

[80] Andersson, H. \& van den Berg, A. (2003). Sensor. Actuat. B-Chem 92 (3), pp. 315-325.

[81] Le Gac, S. \& van den Berg, A. (2010). Trends Biotechnol. 28 (2), pp. 55-62. 


\section{Chapter 6}

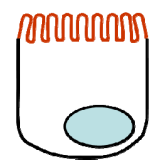

\section{Cell membrane heterogeneities affect the}

\section{outcome of electroporation: implications for in vivo treatment in a clinical setting}

This chapter approaches the use of BLM to gain insight in the effect of the membrane composition on the pore formation process from a slightly different angle than the two previous chapters and focuses, next to the biophysical aspects of more complex models, also on the possible consequences and applications of these measurements for in vivo electroporation. In vivo electroporation is gaining in popularity, particularly as a modality for cancer treatment using electrochemotherapy or a purely electrical treatment. However, in the case of in vivo electroporation, cells are in their natural environment, which influences their membrane organization and constitution. Heterogeneities are found in the membrane composition either between different cells in a tissue (e.g., in a tumour) or within a single cell (e.g., polarized cell), and these heterogeneities are likely to affect the cell response to electroporation. This impact is measured here using BLMs whose composition is varied to recapitulate the two types of heterogeneities and by measuring the onset voltage for pore formation $\left(V_{t h}\right)$. The apical and basolateral membranes of MDCK cells are firstly considered followed by membranes of healthy liver (Moriyama rat) and hepatoma cells (ascite hepatoma AH 130) of rats. Interestingly, MDCK cells (basolateral and apical sides) and liver cells (healthy and cancer) present similar behaviours: cytoplasmic leaflets give identical $V_{\text {th }}$ values while the exoplasmic leaflets of both the apical part of MDCK cells and hepatoma cells better resist electroporation compared to the basolateral part and healthy liver cells, respectively. These results suggest that the protection mechanism of cells to defend themselves against external stress also shields the cell against electrical pulses used for electroporation. 


\section{Introduction}

The introduction of foreign material into cells which is highly relevant for a number of applications, requires rupturing the membrane barrier. A number of in vitro techniques have been reported to achieve this, such as viral delivery, chemical poration, particle bombardment, microinjection, and electroporation [1-9]. The last technique, electroporation, is a universal technique since it is applicable for a great variety of cells and for the introduction of theoretically any compound. In this technique, pores are created in the cell membrane through the application of an external electric field in the form of short high voltage pulses. Thereby, the membrane becomes charged [9] and the transmembrane potential progressively increases. As soon as it reaches the critical value of $0.2-1 \mathrm{~V}$, pores form in the membrane, enabling transport across the plasma membrane [10-11]. As long as the protocol remains mild (low electric field, short pulses) and the parameters are chosen properly, the increase in permeability is transient and pores reseal (reversible electroporation). On the contrary, when the treatment is too harsh (too long or too high voltage), cells cannot recover anymore and they die (irreversible electroporation) [12-13].

Electroporation has firstly been developed for in vitro applications. There, a cell suspension (typically $1 \mathrm{~mL}$ or 1 million cells) is placed in a cuvette equipped with two electrodes on which a high voltage (in the $\mathrm{kV}$ range) is applied. This conventional approach has proven to be successful for the transfer of genes, drugs and particles into many different cell types [14-15] as well as for sterilization purposes with the irreversible electroporation of bacteria [12]. However, cells must be isolated from their natural environment and placed in suspension for their treatment. This limits the applicability of the technique to cells that can easily be retrieved from their natural environment, electroporated and placed back later. Some cells (from the bone marrow or the brain, for instance) cannot easily be extracted and manipulated in vitro, so that they cannot benefit from the technique of electroporation. In that latter case, the treatment must be performed in vivo.

The popularity of the technique of electroporation for the treatment of the skin or tissues close to it has been increasing tremendously over the last two decades [16], to eventually lead to the commercialization of dedicated instrumentation [17]. Two main trends are observed for in vivo electroporation [16, 18]: (i) a purely electrical treatment and (ii) a combined electrical and molecular treatment (e.g., electrochemotherapy or gene transfection). The first type of treatment aims at killing cells by using a very strong electric field that leads to irreversible electroporation [19-21]. A main advantage of this approach is that it does not require any cytotoxic drugs which have a number of undesired side effects on the body. Electrochemotherapy consists of enhancing drug delivery in cells with the assistance of an electric field [16, 18, 22]. This technique has mainly been demonstrated for two small hydrophilic drugs, bleomycin and cisplatinum [23-24], whose uptake by cells is otherwise limited. When combined with electric pulses, not only the cytotoxicity level increases by a factor of 8000 for bleomycin against 80 for cisplatinum [23-24], but the 
treatment also becomes more local: the drug is injected in the tumor before the electrical pulses are applied to promote its entry in the tumour cells [16-18, 22, 25]. Another popular application of a combined electrical and molecular treatment is in vivo gene transfection to remedy the lack of protein expression by the organism or the production of dysfunctioning proteins [16]. Although this method is still at its infancy and has not been applied in humans yet, gene expression has been shown to be maintained for up to nine months in muscle cells in various animal models [26]. Such a long-term expression could ultimately help to decrease the effect of disorders caused by the mutation of genes, such as in the case of cystic fibrosis [18]. The two types of electroporation-based treatment employ the same equipment and share the same issue which is the accessibility of the tissue to be treated. Consequently, electroporation-based treatment is for now mainly demonstrated on skin and subcutaneous tissues which are easily reachable to the electrodes. However, since the technique has proven to be successful for treating dermal cancers in humans [22] and as other tissues are also responsive to a such a modality, other electrode systems are currently under development for the treatment of internal cancers or the transfection of internal tissues [18, 27].

In vivo electroporation presents a key-difference with its in vitro alternative which is operated on isolated cells placed in an artificial context. In an in vivo protocol, this natural environment of the cell must be taken into account, and we discuss here two examples of cases where the cellular context has an influence on the electroporation outcome.

Firstly, the composition of the cell membrane, in vivo, is not always homogeneous but organized in domains. For instance, cells may be polarized and present two parts with distinct (phospho)lipid and protein compositions (see figure 6.1A). These two parts are well defined and separated from each other at the point where two neighboring cells connect (socalled tight-junctions) [28]. It has already been shown that the two halves of the membrane do not exhibit the same behavior with respect to water permeability [29-30], and a comparable difference is to be expected for the response of cells to the application of an external electric field. Moreover, this polarization effect is often accompanied by clustering of proteins in one region of the membrane, and again the amount of proteins affects the resistance of the membrane to pore formation [31-32]. Lastly, lipids and proteins in the cell membranes may be organized in (nano-)domains (e.g. lipid rafts) which are characterized with a higher molecular packing density [33]. As before, the presence of such domains impacts on the membrane response to the application of an electric field [34]. The existence of these different types of heterogeneities in the cell membrane is likely to affect the outcome of the electroporation treatment depending on the orientation of the cell in the electric field. By identifying the importance of this, the protocol can be adjusted to target the regions with a lower resistance to the electric field and to control with a greater accuracy the efficacy of the treatment.

Secondly, in vivo, cells are part of complex structures combining several cell types with different membrane compositions (see figure 6.1B). A particular and highly relevant 
example of this is a tumor tissue where healthy and cancer cells are found in close vicinity with each other. It is well-known that the composition of the cell membrane is altered when a cell becomes cancerous, with for instance noticeable changes in the respective amounts in sphingolipids, cholesterol and glycerolipids, and this affects the membrane properties. An electroporation-based treatment in that case would target cancer cells and rely on either modality discussed before (electrochemotherapy, direct cell killing). As the precise (phospho)lipid composition impacts on the outcome of an electroporation experiment [34], the alteration of the cell membrane composition in cancer can greatly affect the success of the treatment. Two distinct scenarios can be envisioned when a tissue is electroporated in vivo. In a first case, the surrounding healthy cells are easier to electroporate than the actually targeted cancer cells. This is expected when the cholesterol content in the cell membrane becomes higher[34], as is the case, for instance, for hepatoma cells [35]. As a consequence, the treatment is not successful as either the surrounding cells take up the drugs or genes (mild electroporation protocol) or alternatively, they die while the targeted cancer cells are electroporated (harsher electroporation protocol). In a second scenario, the targeted cells indeed respond to the applied electric field before their neighboring cells, and the treatment is successful.

\section{A Heterogeneity in a cell membrane Polarized cells}

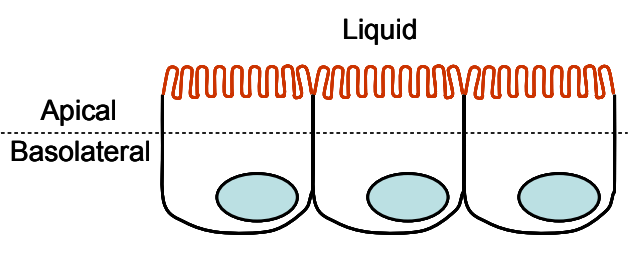

Other cells

\section{B Heterogeneity in a tissue Hepatoma: healthy and cancer cells}

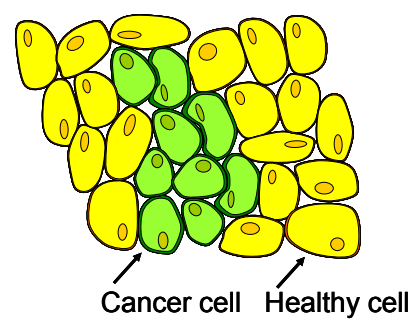

Figure 6.1. Membrane heterogeneities within a cell (left) or in a tissue (right).

Left: Heterogeneities within a cell. Epithelial cells like MDCK cells (Madin-Darby Canine Kidney cells) are polarized and comprise of two parts; the apical part is in contact with the exterior of the body (lumen of capillary for instance) while the basolateral part is connected to the rest of the tissue. The membrane of these polarized cells is also dissimilar in the two parts.

Right: Heterogeneities in a tissue. A tissue is formed of cells belonging to various cell types and having consequently different membrane compositions. For instance, a tumor tissue comprise both healthy and cancer cells, whose membrane composition is altered. As a result, the membrane properties of cancer cells are also modified.

One manner to better appreciate the importance of these various heterogeneities, and to better design in vivo electroporation protocols, consists of thoroughly investigating the response of relevant model membranes to the application of an electric field. We propose here to consider the two possible classes of membrane heterogeneities found in vivo, (i) for polarized cells and (ii) in tumor tissues combining both healthy and cancer cells (see figure 
6.1). For that purpose, BLMs whose compositions reproduce those of real cell membranes are employed. BLMs are ideal platforms for such investigations since their composition is easily modified. The precise (phospho)lipid compositions for the different membrane models studied here are firstly calculated from former reports in the literature as explained in detail in the "results" section of this chapter. The membrane resistance to pore formation is determined by measuring the electroporation threshold $\left(V_{t h}\right)$, the potential at which pores appear in the membranes [7, 34]. In a first series of experiments, polarized MDCK (MadinDarby Canine Kidney) cells are studied, and both their apical and basolateral parts modeled. Following this, the response of healthy liver cells (Moriyama rat liver cells) and hepatoma cells (rat ascite hepatoma AH 130) is compared. Hepatoma cells differ from their healthy analogues by a higher content of cholesterol [35], and this alteration in the membrane composition is known to have a large effect on the membrane's response to electroporation $[7,36]$. For every experiment, the (phospho)lipid compositions of both the inner and outer leaflets of the four different systems considered here (apical and basolateral parts of MDCK, rat ascite hepatoma cells AH 130 and Moriyama rat healthy rat liver cells) are examined.

\section{Materials and Methods}

\section{A. Chemicals}

Lipids (bovine heart 1- $\alpha$-phosphatidylcholine (PC), chicken egg sphingomyelin (SM), porcine brain total cerebrosides (TCB) bovine heart 1- $\alpha$-phosphatidylethanolamine (PE), bovine liver 1- $\alpha$-phosphatidylinositol (PI), porcine brain 1- $\alpha$-phosphatidylserine (PS) and cholesterol (Ch) are purchased from Avanti Polar Lipids (Alabaster, AL). KCl, n-decane and chloroform are purchased from Sigma-Aldrich (St. Louis, MO). Hepes is purchased from Merck Chemicals (Darmstadt, Germany). Deionized water $(18.2 \mathrm{~m} \Omega \times \mathrm{cm}$ ) which is used for all solution preparation and cleaning procedure is obtained from a MilliQ system (Millipore, Billerica, MA).

\section{B. Measurement set-up}

A conventional bilayer system (Warner Instruments, Hamden, CT) is used for BLM experimentation. This system comprises of a delrin cup and chamber containing two round compartments. The cup is inserted in the trans-compartment of the chamber, and the compartments are connected with each other via a $150 \mu \mathrm{m}$ aperture in the cup, across which BLMs are prepared. Both compartments contain $1 \mathrm{~mL}$ buffer solution (10 mM Hepes, $1 \mathrm{M}$ $\mathrm{KCl}, \mathrm{pH}$ 7.4) and $\mathrm{Ag} / \mathrm{AgCl}$ electrodes (used for the electrical characterization of the BLMs). Electrical measurements are carried out with an Axopatch 200b amplifier (Molecular devices, Sunnyvale, CA), applying voltages and measuring currents across the bilayer. Data acquisition is performed with LabVIEW and a PCI-6259 data acquisition card (National Instruments, Austin, TX). All measurements are performed at room temperature. 


\section{Preparation and characterization of BLMs}

All lipids are used as chloroform-based solutions. All (phospho)lipids except TCB and cholesterol are purchased as $10 \mathrm{mg} / \mathrm{mL}$ solutions in chloroform. Cholesterol is purchased as a powder and dissolved in chloroform to yield a $25 \mathrm{mg} / \mathrm{mL}$ solution. Before the preparation of BLMs, a few tenths of $\mu \mathrm{L}$ of the lipid solution in chloroform which are let evaporate overnight yielding an amount of $250 \mu \mathrm{g}$ dried lipids, and subsequently dissolved in ndecane at a final concentration of $25 \mathrm{mg} / \mathrm{mL}$. To improve the solubility of sphingomyelin and cholesterol 2-3\% ethanol can be added. The resulting $n$-decane solutions are used for the preparation of BLMs.

Bilayer lipid membranes are created using the Mueller-Rudin technique [37]. Their formation is monitored using the Axopatch amplifier and characterized by a drop in the current due to the G $\Omega$ seal between the bilayer and the aperture. Thereafter, the membrane capacitance is measured as previously described to confirm the formation of a BLM [38].

\section{Determination of the electroporation threshold of BLMs}

The electroporation threshold $\left(V_{t h}\right)$ of the membrane is defined as the voltage at which the membrane conductivity increases due to the creation of pore(s). This value is determined as the value at which a leakage current is measured through the membrane. For that purpose, a DC voltage is applied to the membrane using the Axopatch amplifier and gradually increased (every $30 \mathrm{sec}$ ) by steps of $20 \mathrm{mV}$, until peaks larger than three times the root mean-square of the noise are observed in the measured current. Pore formation is checked by re-applying the voltage at which peaks are detected; this confirms that the peaks are not due to imperfections in the membrane. The voltage value found corresponds to the electroporation threshold $V_{\text {th }}$. For every membrane composition, $V_{\text {th }}$ is determined at least 3 independent times and the indicated value corresponds to the average of the separate measurements.

\section{Results}

The overall aim of this work is to investigate membrane heterogeneities found (i) within a single cell or (ii) in a tissue, as well as the consequences of these heterogeneities on the outcome of the electroporation process. These two types of heterogeneities are studied here in two successive series of experiments.

Firstly, as a model for cells possessing a partitioned or "organized" membrane, polarized epithelial MDCK cells are employed. These cells present an apical side and a basolateral side which are separated by tight-junctions; the former apical side is exposed to a flow while the latter basolateral one is facing other cells in the tissue. The two sides of the cell membrane are characterized by a different composition [28, 39]. Their properties are also distinct; this has been shown for instance for water permeability, where the apical membrane is the strongest barrier [29-30]. Consequently, their response to the application of an electric field is expected to be different. Secondly, as an example of heterogeneous tissue, 
a tumor (hepatoma) which comprises of both healthy and cancer cells, is considered. In the case of cancer, the cell membrane composition is altered [40], which is very likely to affect their resistance to electroporation. This model of tumor cells is highly relevant for electroporation-based treatment (electrochemotherapy) and clinical applications, and these alterations in the membrane composition need to be examined when designing a treatment protocol for complex tissues so that only one type of cells responds positively to the treatment. For instance, if cancer cells are going to be killed by the treatment, healthy cells must remain unaffected.

\section{A. MDCK cells}

\section{(i) MDCK cells. Apical membrane part}

In this first section, we examine the differences in the measured electroporation threshold voltages for BLMs mimicking the exo- and cytoplasmic leaflets of the apical membrane of MDCK cells. The two leaflets are studied separately as they present distinct compositions and behaviors, as reported for instance for their permeability to water [41-42]. Furthermore, our previous results showed that a difference in the membrane composition can have a large effect on the threshold voltage for electroporation [34]. Since the phospholipid composition of the individual leaflets is very dissimilar [41], a difference in the barrier properties between the two leaflets is expected, while it is not obvious how large this difference is and how this would influence the behavior of the whole membrane. Consequently, both leaflets must be examined separately. Alternatively, an average composition of the two leaflets could be employed, but this would again not give relevant information on the behavior of the asymmetric system.

The phospholipid compositions calculated by Hill et al. are employed to prepare models for the two leaflets of the apical part of the membrane (see Table 6.1) [41]. The resulting BLMs for the exo- and cytoplasmic leaflets give $V_{\text {th }}$ values of $315 \pm 19 \mathrm{mV}$ and $232 \pm$ $18 \mathrm{mV}$, respectively (see figure 6.2). As expected, the outer leaflet is more resistant to pore formation than the inner leaflet, forming thereby the main protective barrier for the cell. These results are in good agreement with the findings of Hill et al. on water and solute permeability [41] for which the exoplasmic leaflet forms a stronger barrier.

\section{(ii) MDCK cells. Basolateral membrane part}

In a second stage, the basolateral part of MDCK cells is considered and the compositions of the two leaflets are determined using the whole membrane composition of MDCK cells, [28, 43-46], using the same assumptions Hill et al. employed to calculate the leaflet compositions of the apical side [41], as detailed below:

1. The ratio in surface area between the apical and the basolateral membranes is $1: 4$ [45].

2. TCB is distributed between the apical and the basolateral part with a 2:1 ratio [46].

3. The cholesterol/phospholipid ratio is $1: 1$ in the apical part against $0.61: 1$ in the basolateral part [44]. 
4. TCB, SM and PC are found only in the exoplasmic leaflet of the membrane [41].

5. PE, PS and PI are found only in the cytoplasmic leaflet of the membrane [41].

Table 6.1. Membrane compositions

Membrane compositions calculated for the electroporation experiments. Precise amount (mol \%) in the different components (phospholipids and cholesterol) employed for the BLMs mimicking the two leaflets of MDCK cells (basolateral and apical parts) and of liver cells (healthy and hepatoma).

\begin{tabular}{|c|c|c|c|c|c|c|c|c|}
\cline { 3 - 10 } \multicolumn{2}{c}{} & \multicolumn{7}{c|}{ Leaflet constituents } \\
\hline Cell membrane type & Leaflet & \% PC & \% SM & \% TCB & $\%$ Ch & $\%$ PE & $\%$ PS & $\%$ PI \\
\hline MDCK: apical & Exoplasmic & 8.6 & 18.4 & 20.8 & 52.3 & - & - & - \\
\hline MDCK: apical & Cytoplasmic & - & - & - & 37.3 & 40.2 & 20.1 & 2.5 \\
\hline MDCK: basolateral & Exoplasmic & 35.4 & 19.1 & 2.6 & 42.8 & - & - & - \\
\hline MDCK: basolateral & Cytoplasmic & - & - & - & 31.8 & 41.1 & 23.2 & 3.9 \\
\hline Liver & Exoplasmic & 63.1 & 16.4 & - & 20.4 & - & - & - \\
\hline Liver & Cytoplasmic & - & - & - & 58.4 & 25.5 & 10.4 & 5.7 \\
\hline Hepatoma & Exoplasmic & 42.0 & 17.2 & - & 40.0 & - & - & - \\
\hline Hepatoma & Cytoplasmic & - & - & - & 43.8 & 38.5 & 15.0 & 2.7 \\
\hline
\end{tabular}

PC: L- $\alpha$-phosphatidylcholine; SM: sphingomyelin; TCB: total cerebrosides; Ch: cholesterol; PE: $L-\alpha$-phosphatidylethanolamine; PS: L- $\alpha$-phosphatidylserine; PI: $L-\alpha$-phosphatidylinositol

The resulting precise lipid compositions for both leaflets are presented in Table 6.1. Large differences are found in the leaflet compositions for the apical and basolateral sides of the membrane. The exoplasmic leaflet of the basolateral part contains far less TCB, less cholesterol, a lot more PC than the apical leaflet, but similar amount of SM. On other aspects, the composition of the cytoplasmic leaflet was expected to be the same as (phospho)lipids can freely diffuse across the tight-junctions between the apical and basolateral sides of the cell membrane [28]. However, slight differences are found: the basolateral side contains less cholesterol, slightly more PS and PI than the apical side, but similar amounts of PE. As before, the electroporation voltage is determined for both leaflets, and those are $265 \pm 19 \mathrm{mV}$ for the exoplasmic side and $235 \pm 10 \mathrm{mV}$ for the cytoplasmic side (see figure 6.2). These two values for $V_{\text {th }}$ are comparable although the cytoplasmic leaflet seems to give a slightly higher value.

A comparison with the values obtained for the apical part show a noticeable difference, especially for the outer leaflet. The inner leaflets present the same resistance to the applied electric field ( $232 \pm 18$ and $235 \pm 10 \mathrm{mV}$ for the apical and basolateral parts, respectively) although the inner leaflet composition was not found identical. The outer leaflets, on the contrary, exhibit very different behaviors $(315 \pm 19$ and $265 \pm 19 \mathrm{mV}$ for the apical and 
basolateral parts, respectively) with the apical part being much more resistant. This difference could be expected as the apical side of the cell is facing a lumen in the body and is more exposed than the basolateral side. As a consequence, it must better resist external aggressions (such as fluid flow), and this is measured here as a higher resistance to the application of an electric field. The basolateral does not need to protect itself against external factors, and its membrane is less strong. On other aspects, this finding is in good agreement with the report of Farinas et al. and Timbs et al., on the water permeability of the apical and basolateral sides of the membrane [29-30].

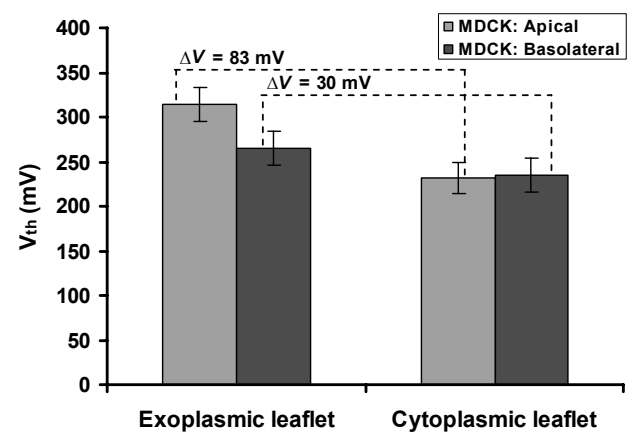

Figure 6.2. Electroporation results MDCK cells

Measured electroporation threshold voltages for the cytoplasmic and exoplasmic leaflets of MDCK cells (apical and basolateral parts).

\section{B. Hepatoma tissue}

\section{(i) Determination of the membrane leaflet compositions}

The other class of heterogeneities found between different cells in a tissue is examined in this second series of experiments that aims to compare the response of healthy liver cells and hepatoma cells to the application of an electric field. This case is particularly relevant for clinical applications where tumor cells are targeted in a treatment based on electrochemotherapy.

As before, the leaflet compositions are calculated for healthy (Moriyama rat cells) and cancer (rat ascite hepatoma $\mathrm{AH} 130$ cells) liver cells using the reported phospholipid composition for the whole membrane of these cells [35, 47] and after having made several assumptions as before:

1. Half of the lipid moles are in each leaflet.

2. Only the main types of (phospho)lipids found in cell membranes are included: phosphatidylcholine (PC), phosphatidylethanolamine (PE), phosphatidylinositol (PI), phosphatidylserine (PS), sphingomyelin (SM), total cerebrosides (TCB), and cholesterol (Ch). 
3. The exoplasmic leaflet contains only PC, SM, TCB and Ch whereas the cytoplasmic leaflet contains only PE, PS, PI and Ch.

4. Phosphatidic acid (PA) is a glycerolipid found in the cytoplasmic leaflet [48]. It contains two hydrocarbon chains, so its molar concentration is added to PE, since this is the most abundant glycerolipid in the cytoplasmic leaflet.

5. Cardiolipin is a glycerolipid that is found in equal ratios in the exo- and cytoplasmic leaflets [48] and contains four hydrocarbon chains, so its molar concentration is added both to PC and to PE.

6. The molar concentrations of lyso-PC and lyso-PE (which differ from PC and PE by the fact that they only present only one hydrocarbon chain) are divided by two and added to $\mathrm{PC}$ and PE, respectively.

The resulting phospholipid compositions per leaflet are shown in Table 6.1. For the liver and hepatoma cells no data is found on the glycolipid content of the membranes and consequently its presence in the outer leaflet cannot be taken into account.

Striking differences are found between healthy liver and hepatoma cells, in the same way as for the apical and basolateral sides of MDCK cells. For the inner leaflet, the cholesterol amount is higher for healthy cells (58.4 vs. $43.8 \%$ ) as well as the amount in PI (5.7 vs. $2.7 \%$ ), while those in PE and PS are lower (25.5 and $10.4 \%$ vs. 38.5 and $15 \%$ ). When comparing the total amount of glycerolipids and cholesterol in these leaflets (41.6 and 58.4\%, respectively, for healthy cells vs. 56.2 and $43.8 \%$, respectively, for hepatoma cells), no large difference is found in the phospholipid compositions which is similar to the cytoplasmic leaflets of the apical and basolateral sides of MDCK cells. The exoplasmic compositions give larger differences, the greatest being the cholesterol amount which is much higher in the case of cancer cells (40 vs. 20.4\%). PC is less present in cancer cells (42.8 vs. $63.1 \%$ ) and the amount of SM remains unchanged (17.2 vs. $16.4 \%$ ). As for the MDCK cells, the differences in the phospholipid composition for both exoplasmic leaflets are much larger than for the cytoplasmic leaflets.

\section{(ii) Electroporation experiments}

These membrane compositions are subsequently employed to prepare model membranes for the four different leaflets. Healthy cells give $V_{\text {th }}$ values of $175 \pm 19$ and $160 \pm$ $10 \mathrm{mV}$ for their exo- and cytoplasmic leaflets, respectively, and cancer cells $220 \pm 16$ and 160 $\pm 10 \mathrm{mV}$ (see figure 6.3).

Firstly, a slight difference of $15 \mathrm{mV}$ only is found in the $V_{\text {th }}$ values measured for both leaflets in healthy liver cells. The asymmetry in the membrane composition between the two leaflets does not seem to affect its resistance which is comparable for both leaflets. For hepatoma cells, the $V_{\text {th }}$ values for the two leaflets are divergent and separated by $60 \mathrm{mV}$, the outer leaflet being more resistant to the application of an electric field. A comparison between healthy and cancer liver cells does not reveal any difference between the inner 
leaflets in terms of the measured $V_{t h}(160 \mathrm{mV}$ in both cases) although the precise composition of the inner leaflets is different, with a higher cholesterol amount in healthy liver cells (58.4\%) compared to hepatoma cells (43.8\%). On the contrary, a $45-\mathrm{mV}$ increase in $V_{\text {th }}$ is determined for the outer leaflets of hepatoma cells compared to healthy liver cells. This difference obviously reflects the two-fold increase in the cholesterol amount in the outer leaflets ( $40 \%$ for cancer cells vs. $20.4 \%$ for healthy cells), as already proposed in former studies by others [40] and ourselves [34].

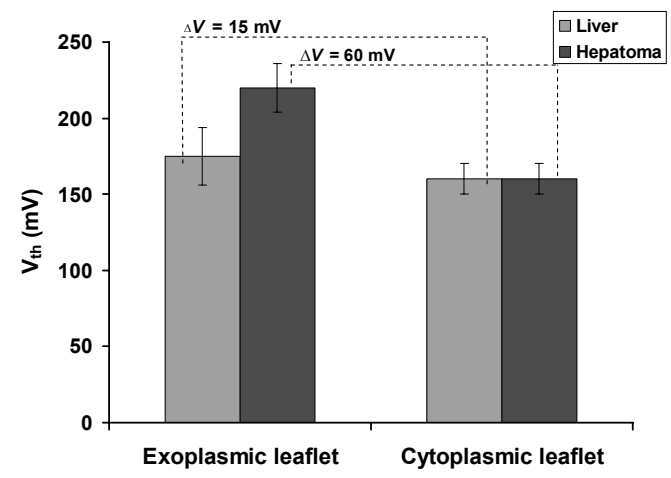

Figure 6.3. Electroporation results liver cells

Measured electroporation threshold voltages for the cytoplasmic and exoplasmic leaflets of healthy liver and hepatoma cells.

\section{Discussion}

\section{A. Membrane composition and electroporation}

Large differences are found in the phospholipid composition of the two leaflets of the different cell lines considered here (see Table 6.1) which translates into large discrepancies in the measured $V_{\text {th }}$ values for the BLMs recapitulating these different leaflets. These findings corroborate our previous work that demonstrates that small variations in the membrane composition have a large effect on its resistance to electroporation [34]. The exoplasmic leaflets of the apical membrane of MDCK cells and hepatoma cells give the largest differences. Two factors may account for this according to our previous results [34]; the larger amount of cholesterol and the ternary composition from a glycerolipid, a sphingolipid and cholesterol in a ratio that gives rise to phase separation phenomena. On the contrary, in the cytoplasmic leaflets that comprise of binary mixtures of glycerolipids and cholesterol, the composition differences have no effect on the measured $V_{t h}$. For MDCK cells, these composition differences are very small and can be neglected while for the liver cells, the phospholipid mixtures are very dissimilar (see Table 6.1) so that one would expect a change in the threshold voltage for electroporation. Nevertheless, the measured $V_{\text {th }}$ values 
are the same $(160 \pm 10 \mathrm{mV})$ for the cytoplasmic leaflets of both liver cells. They only differ from each other by the phospholipid/cholesterol ratio (PL/Ch) (0.71 vs., 1.28, respectively), and according to our previous results, presented in chapter 5 [34], below a PL/Ch value of $1.5, V_{\text {th }}$ remains constant for a binary L- $\alpha-\mathrm{PC} / \mathrm{Ch}$ system. This explains consequently the absence of difference found for both cytoplasmic leaflets.

In chapter 5 , we have characterized the influence of the composition of ternary systems composed of a glycerolipid (L- $\alpha$-PC), a sphingolipid (SM) and cholesterol on the membrane resistance to EP [34], and this gave rise to the establishment of a map of $V_{\text {th }}$ as a function of the exact composition of the ternary system. In this line, it would be interesting to see whether our previous data could be employed to predict the behaviour of a cell membrane in an electroporation-based treatment and if a $\mathrm{L}-\alpha-\mathrm{PC} / \mathrm{SM} / \mathrm{Ch}$ ternary model is a suitable minimal model for the lipid matrix of all cell membranes. Firstly, the $V_{\text {th }}$ values measured for the cytoplasmic leaflets, which are only composed of glycerolipids and cholesterol, are more distinct than those obtained for L- $\alpha$-PC/Ch binary models [34]. For the cytoplasmic leaflets of the apical and basolateral membrane of MDCK cells, the measured average $V_{\text {th }}$ values are $230 \mathrm{mV}$ and $235 \mathrm{mV}$ (see figure 6.2), respectively, while the corresponding models (63/37 L$\alpha$-PC/Ch and $68 / 32 \mathrm{~L}-\alpha$-PC/Ch) would give the values of $\sim 325 \mathrm{mV}$ and $\sim 200 \mathrm{mV}$, respectively. Similarly, for the liver cells (cytoplasmic leaflet), a $V_{\text {th }}$ value of $160 \mathrm{mV}$ (see figure 6.3) is measured against $\sim 410 \mathrm{mV}$ for both membrane models (42/58 L- $\alpha$-PC/Ch and $56 / 44 \mathrm{~L}-\alpha$ $\mathrm{PC} / \mathrm{Ch}$ ). These results show that $\mathrm{L}-\alpha-\mathrm{PC}$ is not a good model for all glycerolipids, as first hypothesized from the results on the binary phospholipid mixtures obtained in chapter 4 . There, changing the phospholipid composition of membranes whose basic building block consisted of DPhPC did not have a large effect. However, $\mathrm{L}-\alpha-\mathrm{PC}$ has a completely different structure than DPhPC and the structural properties of L- $\alpha-\mathrm{PC}$, on one hand, and of PS, PI and PE (actually present in the cytoplasmic leaflets), on the other hand, are also very different: L- $\alpha$-PC has a smaller headgroup than PI and PS and less unsaturations than PE which is the predominant glycerolipid in the membrane. In other words, a reliable minimal model for the cytoplasmic leaflet of cell membrane would employ two glycerolipids, one with more unsaturations (e.g., PE) and one with a larger headgroup, together with cholesterol. Secondly, the measured $V_{\text {th }}$ values for the exoplasmic leaflets are compared to the values obtained for ternary $\mathrm{L}-\alpha-\mathrm{PC} / \mathrm{SM} / \mathrm{Ch}$ models. Since no data is found on the glycolipids content of the liver membranes, the liver cell membrane models are already composed of a ternary L- $\alpha$-PC/SM/Ch mixture so that they are not considered here. MDCK cell leaflets also contain the sphingolipid TCB which is added to the SM amount. The measured average $V_{\text {th }}$ values for the apical and basolateral exoplasmic leaflets are $315 \mathrm{mV}$ and $265 \mathrm{mV}$ (see figure 6.2), respectively, while the corresponding models (9/39/52 and $35 / 22 / 43 \mathrm{~L}-\alpha-\mathrm{PC} / \mathrm{SM} / \mathrm{Ch}$, see chapter 5) give the values of $\sim 270 \mathrm{mV}$ and $\sim 260 \mathrm{mV}$, respectively. Interestingly, the difference between the measured and the model values correlate with the TCB amount that seems to impact on the membrane resistance. To study this effect more closely, two more ternary models were tested where TCB is taken as a model for all 
sphingolipids in the membrane. The corresponding BLMs (9/39/52 and 35/22/43 L- $\alpha$ $\mathrm{PC} / \mathrm{TCB} / \mathrm{Ch}$, respectively) give higher $V_{\text {th }}$ values, of $327 \pm 12 \mathrm{mV}$ and $320 \pm 16 \mathrm{mV}$, respectively. This strengthening effect of TCB can be explained by the fact that TCB belongs to the sub-family of glycolipids which are reported to interact strongly with cholesterol through intermolecular hydrogen bonds via their sugar groups [49-50]. Furthermore, TCB possesses two chains of different lengths that interdigitate with the tails of the phospholipids in the opposite leaflet [51]. As a consequence, the addition of TCB in the membranes has a strong stabilizing effect. Again, these results suggest that a $\mathrm{L}-\alpha-\mathrm{PC} / \mathrm{SM} / \mathrm{Ch}$ ternary model is not sufficient to mimic the exoplasmic leaflet of cell membranes and that a minimal model should also include a glycolipid.

\section{B. Membrane composition and electroporation-based treatment}

For in vitro experiments, cells are isolated from the body and, in most cases, treated in suspension. However, MDCK cells loose their polarization in solution and their membrane becomes "homogeneized". Similarly, cancer cells have been reported to loose their cancerness when removed from their environment and cultured in 2D on a surface [52], and this may impact on their precise membrane composition. Consequently, the protocol for in vivo electroporation-based treatment cannot be derived from a protocol developed in vitro on cell in suspension. Here, we propose an alternative approach that consists of considering the differences in response to electroporation by using membrane models recapitulating the precise composition of cells in vivo.

Two models are considered here, MDCK epithelial cells as a model for polarized epithelial cells and liver/hepatoma cells as models of cells found in liver tumor tissues. The most targeted tissue for electroporation-based is the skin which comprises of epithelial cells. Consequently, the MDCK cells employed in this work are relevant models for the outer layer of the skin. By studying their membrane properties, a good knowledge can be acquired on the behavior of polarized cells under the application of an electric field, and what the consequence is of this polarization. Our results indicate that pore formation is favored on the basolateral side, and is most likely to start on that side of the cells. The experiments on liver cells show that hepatoma cells are much more resistant to pore formation compared to healthy liver cells. Consequently, when treating this tissue in vivo special precautions must be taken to prevent the healthy cells from taking up drugs and being killed. However, it is worth mentioning that only one type of cancer is considered here, and other cancer cells can present other alteration patterns in their membrane composition [40]. A higher amount of cholesterol as here strengthens the barrier properties of a cell, but other cancer cells may exhibit lower cholesterol content in their membrane [40] or very different phospholipid ratios.

As a conclusion, taking a closer look at the precise membrane composition of cells when designing an in vivo or in vitro treatment protocol can help in providing insight into how they and their neighboring cells would respond to the electric field. The latter approach 
is taken in chapter 8 , where the response to electroporation is studied of monolayers of MDCK cells that are grown onto two different substrates that induce or not membrane polarization. This understanding will help to design better, more accurate and bettertargeted protocols.

\section{Process of electroporation}

In general, the cytoplasmic leaflets appear to be less resistant to pore formation, and the exoplasmic leaflets give the greatest variations in $V_{\text {th }}$ values. To corroborate our hypothesis that cells having a stronger outer barrier are less likely to become electroporated, the leaflet that is dominating during the pore formation process should be resolved. If pore formation starts on the cytoplasmic side of the membrane, and if the strength of the outer membrane leaflet has no effect on propagation of the pore through the whole membrane, then no difference is expected in the response of cells to the applied electric field between both sides of MDCK cells and between healthy and cancerous liver cells. Answering this question requires the use of asymmetrical membranes that mimic both the cyto- and exoplasmic leaflets. With these models, not only the side where pore formation starts can be determined, but also how the pores propagate depending on the strength of the outer barrier.

\section{Treatment and heterogeneity}

An interesting outcome of these two series of experiments that examine the existence of heterogeneities in the cell membrane compositions is that the two types of heterogeneities considered here appear to be very similar. A parallel can easily be made between, on one hand, basolateral and apical parts of MDCK cells and, on the other hand, healthy and cancer liver cells, as the same behavior is observed for basolateral membranes and healthy liver cell membranes, and for apical membranes and hepatoma cell membranes. Firstly, healthy liver cells can be compared to the basolateral side of MDCK cells, where similar $V_{\text {th }}$ values are measured for both leaflets. Secondly and similarly, hepatoma cells resemble the apical side of MDCK cells in that the outer leaflets are much more resistant to the application of an electric field (+ $60 \mathrm{mV}$ for liver cells vs. $+83 \mathrm{mV}$ for MDCK cells). Thirdly, the inner leaflets show little (MDCK cells, apical vs. basolateral side) or no (healthy liver vs. hepatoma cells) difference in the resistance to the applied electric field while the outer leaflets are much stronger for the apical side of MDCK cells (+ $50 \mathrm{mV}$ ) and hepatoma cells (+ $45 \mathrm{mV}$ ). These two last observations reveal that the barrier properties in the outer leaflet are enhanced while the inner leaflet remains unchanged compared to basolateral and healthy cells. For the apical part of the MDCK cells, this asymmetry in the resistance properties is known to be employed by the cells to reinforce their barrier properties of the side of the cell exposed to external stimuli (among others, shear flow). Similarly, one can expect cancer cells to develop such an asymmetry as a protection mechanism against external attacks. Since their resistance to an applied electric field is related to the structural and molecular properties of the membranes [34], the cells are likely to be more resistant to any form of perturbations, 
and not only in the frame of electroporation. These results are corroborated by AFM studies on cell membrane properties where differences are found in the stiffness of membranes of various healthy and cancer cells [53-54]. These common observations for both polarized MDCK cells and liver/hepatoma cells suggest a generic mechanism adopted by cells to protect themselves against external perturbations: they increase their barrier properties by altering the content of their outer leaflet. Similarly, cells that are not supposed to receive external aggressions would possess two leaflets with comparable resistance and properties. As a summary, the heterogeneities found in cell membranes within a cell or between cells are apparently generated to enhance barrier properties, in general, or as a response or prevention against external aggressions.

\section{Conclusion}

The response of cell membranes to the application of an electric field strongly depends on their composition, as shown here for the cyto- and exoplasmic leaflets of four cell models: the apical and basolateral parts of MDCK cells, healthy liver cells (Moriyama rat) and hepatoma cells (rat ascite AH 130). These results highlight the need to study the cell membrane properties and develop cell-specific protocols before starting a clinical electroporation-based treatment on patients.

However, before the full effect of these differences in $V_{\text {th }}$ of the leaflets can be understood, two questions remain. Where does pore formation during electroporation precisely start? And, what is the probability for the pore to propagate to the other leaflet if the latter is much more resistant? Answering these questions requires experimentation on asymmetrical membranes that would recapitulate the composition of both exo- and cytoplasmic leaflets.

An interesting conclusion of these results is that different types of heterogeneities may have the same origin. Both cell types that are expected to experience a higher level of aggression (shear stress in case of the apical part of MDCK cells and the body defense mechanism in the case of cancer cells) display an increased resistance to pore formation from their exoplasmic leaflet compared to the other leaflets. This increase in the strength of the leaflets is related to a change in their phospholipid composition. As a consequence, by simply altering the phospholipid composition of their membranes, cells are easily able to protect themselves. This suggests that the cell response to increased shear stress (strengthening its exoplasmic membrane leaflet) also provides a "protection" against other perturbations such as electrical pulses used for electroporation.

\section{References}

[1] Adamo, A. \& Jensen, K.F. (2008). Lab Chip 8 (8), pp. 1258-1261.

[2] Chen, X. et al. (2007). Proc. Natl. Acad. Sci. USA 104 (20), pp. 8218-8222.

[3] Graham, F.L. \& Vandereb, A.J. (1973). Virology 52 (2), pp. 456-467.

[4] Klein, T.M. et al. (1987). Nature 327 (6117), pp. 70-73.

[5] Luo, D. \& Saltzman, W.M. (2000). Nat. Biotechnol. 18 (1), pp. 33-37. 
[6] Uchida, M. et al. (2009). BBA 1790 (8), pp. 754-764.

[7] van Uitert, I. et al. (2010). BBA - Biomembranes 1798 (1), pp. 21-31.

[8] Yang, N.S. (1992). Crit. Rev. Biotechnol. 12 (4), pp. 335-356.

[9] Chang, D.C. et al., Guide to Electroporation and Electrofusion. Academic Press, Inc.: San Diego, CA, USA, 1992.

[10] Zimmermann, U. et al. (1974). Biophys. J. 14 (11), pp. 881-899.

[11] Weaver, J.C. \& Chizmadzhev, Y.A. (1996). Bioelectrochem. Bioenerg. 41 (2), pp. 135-160.

[12] Sale, A.J.H. \& Hamilton, W.A. (1967). BBA 148 (3), pp. 781-788\&.

[13] Sale, A.J.H. \& Hamilton, W.A. (1968). BBA 163 (1), pp. 37-43.

[14] Zimmermann, U. et al. (1976). BBA 436 (2), pp. 460-474.

[15] Neumann, E. et al. (1982). EMBO J. 1 (7), pp. 841-845.

[16] Gehl, J. (2003). Acta Physiol. Scand. 177 (4), pp. 437-447.

[17] Sadadcharam, M. et al. (2008). Int. J. Hyperther. 24 (3), pp. 263-273.

[18] Dev, S.B. et al. (2000). Ieee Transactions on Plasma Science 28 (1), pp. 206-223.

[19] Al-Sakere, B. et al. (2007). Plos One 2 (11).

[20] Rubinsky, B. et al. (2007). Technol. Cancer Res.T. 6 (1), pp. 37-48.

[21] Tang, L.L. et al. (2009). Biochem. Biophys. Res. Commun. 390 (4), pp. 1098-1101.

[22] Marty, M. et al. (2006). Ejc Supplements 4 (11), pp. 3-13.

[23] Orlowski, S. et al. (1988). Biochem Pharmacol 37 (24), pp. 4727-33.

[24] Sersa, G. et al. (1995). Cancer Res 55 (15), pp. 3450-5.

[25] Rols, M.P. (2006). BBA-Biomembranes 1758 (3), pp. 423-428.

[26] Mir, L.M. et al. (1998). C R Acad Sci III 321 (11), pp. 893-9.

[27] Soden, D.M. et al. (2006). Cancer Lett. 232 (2), pp. 300-310.

[28] Van Meer, G. \& Simons, K. (1986). EMBO J. 5 (7), pp. 1455-1464.

[29] Farinas, J. et al. (1997). J. Gen. Physiol. 110 (3), pp. 283-96.

[30] Timbs, M.M. \& Spring, K.R. (1996). J Membr Biol 153 (1), pp. 1-11.

[31] Navarrete, E.G. \& Santos-Sacchi, J. (2006). Biophys. J. 90 (3), pp. 967-974.

[32] Troiano, G.C. et al. (1999). Biophys. J. 76 (6), pp. 3150-3157.

[33] Brown, D.A. \& London, E. (2000). J. Biol. Chem. 275 (23), pp. 17221-17224.

[34] van Uitert, I. et al. (2010). Soft Matter 6 (18), pp. 4420-4429.

[35] Koizumi, K. et al. (1976). J. Biochem. 79 (4), pp. 739-748.

[36] Koronkiewicz, S. \& Kalinowski, S. (2004). BBA-Biomembranes 1661 (2), pp. 196-203.

[37] Mueller, P. et al. (1963). J. Phys. Chem 67 (2), pp. 534-535.

[38] Wiese, A. \& Seydel, U. (2000). In Methods in Molecular Biology, pp 355-370.

[39] Van Meer, G. \& Simons, K. (1982). EMBO J. 1 (7), pp. 847-852.

[40] Hendrich, A.B. \& Michalak, K. (2003). Curr. Drug Targets 4 (1), pp. 23-30.

[41] Hill, W.G. \& Zeidel, M.L. (2000). J. Biol. Chem. 275 (39), pp. 30176-30185.

[42] Krylov, A.V. et al. (2001). J. Gen. Physiol. 118 (4), pp. 333-339.

[43] Hansson, G.C. et al. (1986). EMBO J 5 (3), pp. 483-9.

[44] Simons, K. \& van Meer, G. (1988). Biochemistry 27 (17), pp. 6197-202.

[45] van Genderen, I. \& van Meer, G. (1995). J Cell Biol 131 (3), pp. 645-54.

[46] van Meer, G. et al. (1987). J Cell Biol 105 (4), pp. 1623-35.

[47] Koizumi, K. et al. (1977). Cell Struct. Funct. 2 (2), pp. 145-153.

[48] Daleke, D.L. (2007). J. Biol. Chem. 282 (2), pp. 821-825. 
[49] Simons, K. \& Vanmeer, G. (1988). Biochemistry 27 (17), pp. 6197-6202.

[50] Wang, T.Y. \& Silvius, J.R. (2000). Biophys. J. 79 (3), pp. 1478-1489.

[51] Yeagle, P. (1992). The Structure of Biological Membranes.

[52] Smalley, K.S. et al. (2006). In Vitro Cell Dev Biol Anim 42 (8-9), pp. 242-7.

[53] Cai, X. et al. (2010). Micron 41 (3), pp. 257-62.

[54] Li, Q.S. et al. (2008). Biochem. Biophys. Res. Commun. 374 (4), pp. 609-613. 


\section{Chapter 7}

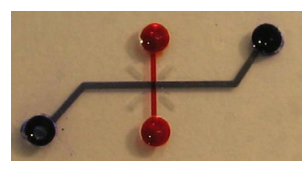

\section{Bilayer lipid membranes on an integrated microfluidic platform}

In this chapter, a completely different approach is taken with respect to the platform applied for BLM experimentation. One conclusion of chapter 3 was that the future trend for BLM experimentation lies in its miniaturization as it notably brings numerous key-advantages for both sensitive electrical and optical measurements. The first two experimental chapters have indeed demonstrated that our study can benefit from this. In this chapter, we examine this issue of miniaturization of BLM experimentation and their implementation in a microfluidic device for both fundamental studies and experimentation concerning proteins. We propose a novel microfluidic device as well as a novel methodology to create BLMs in a closed environment. The resulting BLMs are characterized using both electrical and optical techniques. The system is subsequently employed for early single protein studies on the model channel protein $\alpha$-hemolysin. 


\section{Introduction}

Since cell membranes have a highly complex structure (see chapter 3), simplified models are often employed to study, for instance, their (structural) properties. A commonly used model is a bilayer lipid membrane or BLM \{Mueller, 1963 \#468;Tamm, 1985 \#400;Tien, $2001 \# 421$ \}, a planar model whose composition can be varied to include various phospholipids, cholesterol and proteins. In chapter 3, we have seen that the future trend for BLM experimentation lies in its miniaturization, as this notably brings numerous keyadvantages for both sensitive electrical and optical measurements. Therefore, this last chapter concerning BLM studies discusses a completely different approach to BLM experimentation compared to the previous chapters. Instead of employing the BLMs to study the electroporation process, a novel system is designed specifically for BLM experimentation. One of the driving forces for this miniaturization trend is the interest of the pharmaceutical industry in having multiplexed and miniaturized tools for high-throughput screening of drugs on channel proteins.

However, other fields of applications of BLMs may strongly benefit from the miniaturization and multiplexing of BLM experimentation platforms. For instance, in the previous three chapters, we have described different series of experiments employing BLMs in a conventional experimentation device. All these experimental works would gain a lot when performed in a miniaturized - or even microfluidic - device as explained in more detail in the following sections.

\section{A. Reduction in size of the aperture and the reservoirs}

A conventional set-up includes features with relatively large dimensions such as two large reservoirs $(1.5-2 \mathrm{~mL}$ ) and a large aperture (typically $150 \mu \mathrm{m}$, and up to $1 \mathrm{~mm}$ ), and this is not optimal for a number of studies.

A direct consequence of the large diameter of the aperture is the reduced stability of the membranes [4-5], which precludes experiments for a prolonged period of time. When the aperture is fabricated using micro- and nanotechnology processes, its size can easily be decreased down to several $\mu \mathrm{m}$, and potentially to a few tens of nm [6-7]. A first advantage of employing smaller apertures is a gain in membrane stability. For instance Eray et al. report for the first time a stability of $50 \mathrm{~h}$ for membranes prepared on miniaturized apertures (40 $\mu \mathrm{m})$ compared to a few hours or less in conventional systems [4]. A prolonged lifetime is notably crucial for long experiments where, for instance, a large number of proteins must be inserted in the membranes (See chapter 4).

On other aspects, as the surface area of the membrane is smaller, fewer proteins are required to obtain a certain surface density in proteins. For instance, using a conventional system, we report in chapter 4 a protein density of 0.74 proteins $/ \mu \mathrm{m}^{2}$, which lies $5-6$ orders of magnitude below that found in cell membranes $\left(125 \times 10^{3}\right.$ proteins $\left./ \mu \mathrm{m}^{2}\right)$. Since the density scales with the square of the aperture diameter, a cell membrane-like density could be 
reached using 150-nm diameter apertures produced using nanofabrication techniques, as reviewed in chapter 3 . On the contrary, when working with a smaller aperture of $10 \mu \mathrm{m}$, for instance, only 29 proteins instead of 6500 are required to achieve the same maximum surface density obtained in our study. Another direct consequence is that the experimentation becomes faster as fewer proteins must insert in the membrane.

Downscaling of the reservoirs also contributes to faster experimentation, as the insertion in the membrane is governed by diffusion phenomena. A protein presents a diffusion coefficient in the order of $10^{-11} \mathrm{~m}^{2} / \mathrm{s}$, so in a reservoir of $1-\mathrm{cm}$ diameter, $10^{7} \mathrm{~s}(2,778$ $\mathrm{h}$ or more than 115 days!) are necessary for the protein to reach the membrane if the solution is not stirred against $250 \mathrm{~s}$ (4 min) in a $50 \mu \mathrm{m}$ high microchannel. The same principle applies when other chemicals must be flushed in the system (e.g., drugs to be tested on proteins); refreshment of solutions is much faster and more straightforward than in a conventional setup. All together, the experimentation time is reduced, increasing not only the throughput of the assay but also the probability of a successful outcome (since the risk for membrane rupture decreases).

A second main advantage of the miniaturization of the device is the gain in sensitivity for the electrical measurements. The level of electrical noise directly correlates with the size of the aperture. A large aperture gives higher electrical noise, making the measurements less sensitive [8]. Smaller apertures enable to decrease dramatically the noise level. This not only opens the door to single protein measurements but also facilitates the detection of smaller pores in the membrane for electroporation studies. The size of the reservoirs has also a large influence on the sensitivity of the measurements [9]. The stray capacitance existing between the electrodes and the electrolyte varies with the volume of electrolyte: the larger the volume the higher the electrical noise. Smaller reservoirs are consequently preferred to enhance the sensitivity of the measurements.

A last advantage is the reduced consumption in chemicals, proteins and reagents; large reservoirs have a capacity of $1 \mathrm{~mL}$ while microfluidic devices only have a total volume of a few $\mu \mathrm{L}$ and require some tenths of $\mu \mathrm{L}$ to be filled Finally, when working with a microfluidic device, the reservoirs are closed, which limits evaporation issues of the buffer (membranes break upon prolonged exposure to air).

\section{B. Characterization techniques}

A second main drawback of conventional set-ups is that BLMs are vertical. This limits their characterization to electrical techniques, and prevents, for instance, the use of optical studies that can give substantial additional information on the membrane properties. For instance, the phase separation predicted by electrical measurements for ternary L- $\alpha$ $\mathrm{PC} / \mathrm{SM} / \mathrm{Ch}$ mixtures (chapter 5) can be confirmed by fluorescent techniques [10-13]. Besides, the observations of the location and the possible clustering of protein could bring further knowledge about their effect on the membrane properties (such as its thickness and fluidity), whereas combining the measurement of the membrane surface area with the 
(electrically detected) amount of proteins gives a better indication to their density. Lastly, optical techniques can be employed to detect pore formation, either by proteins or by electroporation or related techniques [14-16]. The addition of a dye on one side of the membrane and the observation of the leakage of the dye through the pores to the other side gives information about both the size and opening times of the pores [14, 16]. Similarly, the functionality of protein channels can be investigated by measuring their ionic permeability as a function of the buffer conditions and the voltage applied on the membrane [17-18].

In most of the cases, in microfluidic devices, membranes are prepared horizontally which makes their dual characterization using both electrical and optical techniques possible. Thereby, the information derived from the electrical measurement can be confirmed by the observation of the membranes using bright field or fluorescence microscopy. This also opens the way to novel and more complex experiments. Horizontal membranes are of interest not only for optical measurements, but also for other types of characterization relying on the use of scanning techniques such as scanning ion conductance microscopy (SICM) or atomic force microscopy (AFM). In this case, the top microfluidic channel must still be replaced by a large open reservoir so that the membrane becomes accessible.

\section{Integration of the experimentation}

A conventional experimentation set-up comprises a single aperture, and only one BLM at a time can be prepared and studied. However, every experiment must be performed at least three times to get statistically relevant data and to check the reproducibility of the results. In addition, for screening tests, a large number of membranes are necessary. In both cases, this increases considerably the time for experimentation. For instance, in chapter 5, we propose electroporation experiments as a novel methodology for establishing ternary diagrams. Although this methodology is much faster than conventional approaches, it still involves a large number of experiments using different membrane compositions. In a multiplexed system, not only several membranes with the same composition can be tested at the same time, but also membranes from different phospholipid mixtures. Thereby, the experimentation is considerably faster, and in a single measurement, the effect of one of the three components can be measured (while the ratio between the other two is kept constant). A more famous example of applications of multiplexed devices is the screening of drugs on membrane proteins; typically, the protein of interest must be exposed to a large number of molecules, preferably in the same conditions, for a first assessment on the activity of the drug.

For these screening tests, most particularly, aperture arrays and multiplexed devices are of great interest. Such devices enable to prepare a number of independent BLMs in parallel, and to perform multiple measurements simultaneously on membranes that have either a similar or a different composition. Consequently, the experimentation time is dramatically reduced especially when both the membrane preparation and the 
measurements are automated. Microfabrication techniques and microfluidics lend themselves well to the preparation of aperture arrays and multiplexed devices, opening the route for routine high-throughput and multiplexed studies on BLMs. In addition to this, when using microfluidic devices, BLM experimentation (formation and measurements) can be automated [5, 19-21], integrated in more complex platforms and even diversified. The flow at the microscale presents unique properties, which brings novel experimentation opportunities [22]. Firstly, solutions can be refreshed quickly via the microfluidic channels, and the experimentation becomes dynamic. Secondly, gradients can easily be generated in a microfluidic device, making concentration-dependent experiments possible in one single step. In the context of BLM experimentation, this ability can be exploited to vary, for instance, the buffer concentration or that in proteins. More interestingly, this generates the option to dynamically prepare the (phospho)lipid mixtures in the chip, by mixing the different components with different relative ratios and by generating gradient between the different (phospho)lipid solutions. Different microfluidic designs lend themselves to the creation of gradients [23]. For instance, a Y-junction can be integrated on a chip (see figure 7.1A), and the solutions with the different compositions isolated at the end of the channel [24]. An alternative design employs a serpentine mixing channels [25] where by splitting, mixing and combining the solutions in the channels a gradient is formed across several separated channels (see figure 7.1B). The advantage of this latter approach is that it takes less space on chip. A key-advantage of preparing the lipid mixtures on the chip is the decreased manipulation of the solution, which often results in loss of material by adsorption on any container.
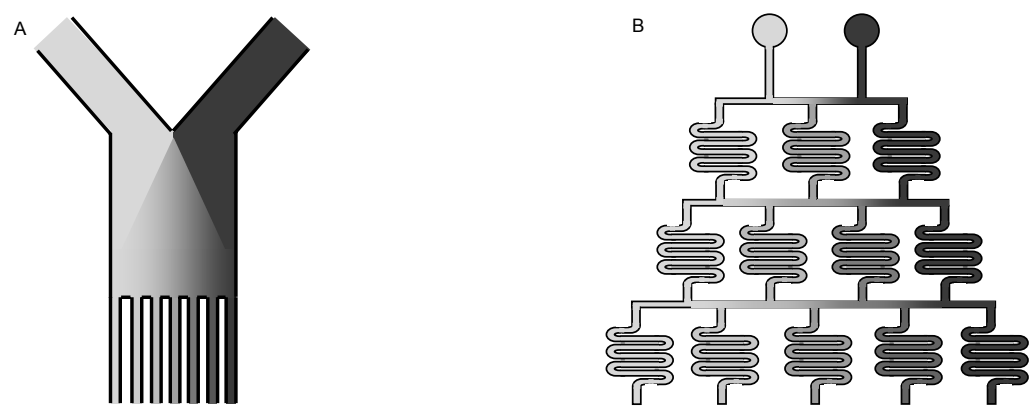

Figure 7.1. Gradient formation.

Schematic representation of the formation of a gradient. A) Gradient formation in a microfluidic channel with the help of a Y-junction. At the end of the channel, solutions with different compositions can be tapped off by the smaller microfluidic channels present there. B) Gradient formation by subsequent combining, mixing and splitting of two different solutions in serpentine channels.

Microfluidic devices are also ideal platforms for automation of processes and they certainly will enable the automation of membrane preparation by monitoring, for instance, the electrical properties of the membranes as discussed later in this chapter. This possible 
automation of the membrane preparation is of great interest, especially when combined with the gradient formation discussed above. This will result in faster and better reproducible experiments, which is a pre-requisite for device commercialization.

A last key-advantage of microfluidic devices for BLM experimentation using native proteins is their enhanced ability to manipulate small-sized entities. In most of the cases, membrane proteins must be kept in a membrane-like environment such as vesicles and the proteins are inserted by fusing the vesicle with the membrane. Microfluidic devices provide for this step not only faster diffusion times to the membrane but also help for the manipulation of the vesicles using dedicated microfluidic tools. Higher fusion rates are consequently expected in a microfluidic device.

In the present chapter, the development of a microfluidic device is presented for miniaturized and integrated BLM application. The design of the device is first discussed in terms of potential upgrade in the future for multiplexed and automated measurements, and its fabrication subsequently detailed. BLM formation relies on a new technique developed in the laboratory that borrows much from techniques developed in the groups of Takeuchi and Morgan [26] The resulting BLMs are characterized using a combination of optical and electrical techniques, and applied for early single protein studies using the model channel protein $\alpha$-hemolysin.

\section{Materials and Methods}

\section{A. Chemicals}

Lipids (1,2-diphytanoyl-sn-glycero-3-phosphocholine (DPhPC) and 1,2-dioleoyl-snglycero-3-phosphoethanolamine-N-(lissamine rhodamine B sulfonyl) (18:1 DOPErhodamine B) are purchased from Avanti Polar Lipids (Alabaster, AL). The optical adhesive NOA-83H is purchased from Norland Products Inc. (Cranbury, New Jersey, USA). Teflon FEP (Fluorinated ethylene propylene) foil (12.5 $\mu \mathrm{m}$ thickness) is purchased from Sabic BV Snij-Unie HiFi (Enkhuizen, the Netherlands). $\mathrm{KCl}$, n-decane, sulfuric acid $\left(\mathrm{H}_{2} \mathrm{SO}_{4}\right)$ and chloroform are purchased from Sigma-Aldrich (St. Louis, MO). Hepes and hydrogen peroxide $\left(\mathrm{H}_{2} \mathrm{O}_{2}\right)$ are purchased from Merck Chemicals (Darmstadt, Germany). Deionized water (DI-water) $(18.2 \mathrm{M} \Omega \times \mathrm{cm})$ which is used for all solution preparation and cleaning procedure is obtained using a MilliQ Analysis system (Millipore, Billerica, MA).

\section{B. Fabrication of the BLM microdevice}

The device for BLM experimentation developed in this work consists of a three-layer sandwich as shown in figure 7.2. The device is composed of two glass layers in which the microfluidic channels and alignment marks are fabricated, and a thin Teflon sheet containing the aperture across which the BLM is formed. Additionally, the top glass substrate includes fluidic accesses (reservoirs), and the Teflon foil access holes (for the fluidic connections with the bottom channels). The fabrication of the device proceeds via the 
separate manufacturing of the three layers as illustrated in the flow charts presented in figures 7.3-6. The two glass parts are produced simultaneously using similar processing steps, whereas the Teflon membrane is fabricated separately. In a last step, the three layers are assembled together to give the final device.

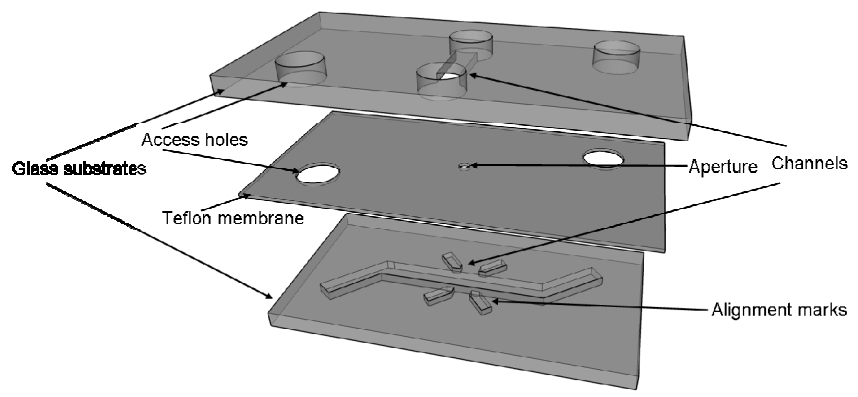

Figure 7.2. Microfluidic device for BLM formation

Schematic drawing showing the different layers of the microfluidic device. The two glass substrates contain the individual microfluidic channels $(200 \mu \mathrm{m} \times 50 \mu \mathrm{m})$ and the top substrate access holes $(1 \mathrm{~mm}$ in diameter) for the fluidic connections. The thin Teflon sheet in the middle (thickness $12.5 \mu \mathrm{m})$ includes the aperture $(10-100 \mu \mathrm{m}$ diameter) and two holes (diameter: $1 \mathrm{~mm}$ ) for the connection of the bottom microfluidic channel to the reservoirs in the top glass substrate.

\section{(i) Processing of the glass substrates}

Two borosilicate glass wafers (Borofloat 33, 100-mm diameter, $500 \mu \mathrm{m}$ thickness) are first thoroughly cleaned in fuming $100 \% \mathrm{HNO}_{3}$ for $10 \mathrm{~min}$, rinsed in deionized water (DI water) and dried (figure 7.3A). In a next step, a chromium-gold layer ( $30 \mathrm{~nm} \mathrm{Cr}$ followed by $150 \mathrm{~nm} \mathrm{Au}$ ) is sputtered (homemade sputtering machine) on the cleaned glass wafers. This layer is patterned with a standard photolithography process. For that purpose, a positive photoresist (OIR 907-17) is spin-coated on the metal bilayer (4000 rpm, $20 \mathrm{~s}$, resulting in a 1.7 $\mu \mathrm{m}$ thick layer) (figure $7.3 \mathrm{~B})$. After a pre-baking step $\left(95^{\circ} \mathrm{C}\right.$ for $\left.1 \mathrm{~min}\right)$, the photoresist is exposed to UV irradiation (Mask aligner EVG-620, $12 \mathrm{~mW} / \mathrm{cm}^{2}$ for $5 \mathrm{~s}$ ), developed in appropriate solvent (OPD 4262 developer), rinsed in DI water and hard-baked $\left(120^{\circ} \mathrm{C}\right.$ for 30 min) (figure 7.3C). A Riston foil is placed on the backside of the wafer to protect it during the rest of the process. The hard mask used for etching the channels and alignment marks in the glass wafers is patterned in the $\mathrm{Cr} / \mathrm{Au}$ layer by etching the exposed areas for a few seconds (Au etchant, 105043; $\mathrm{Cr}$ etchant, 111547.2500) (figure 7.3D). The microstructures are subsequently wet-etched using a 4:1 50\% $\mathrm{HF}: 36 \% \mathrm{HCl}$ solution (BASF) for $19 \mathrm{~min}$ at $18^{\circ} \mathrm{C}$ (figure 7.3E). Since this etching process is isotropic, the glass is also etched underneath the metal mask. Consequently, the resulting structures are wider than the structures patterned in the metal layer, and the channels are designed accordingly to obtain channels with the desired width. In this particular case, a channel width of $100 \mu \mathrm{m}$ in the masking layer results in $200 \mu \mathrm{m}$ wide channels in the glass substrates when etching to a depth of $50 \mu \mathrm{m}$. 
Thereafter, the wafers are cleaned in DI water, the remaining photoresist removed with acetone (BASF) and the Riston foil peeled off. The $\mathrm{Cr} / \mathrm{Au}$ layer is also removed using the same etchants as before, and the wafers rinsed a last time in DI water (figure 7.3F).

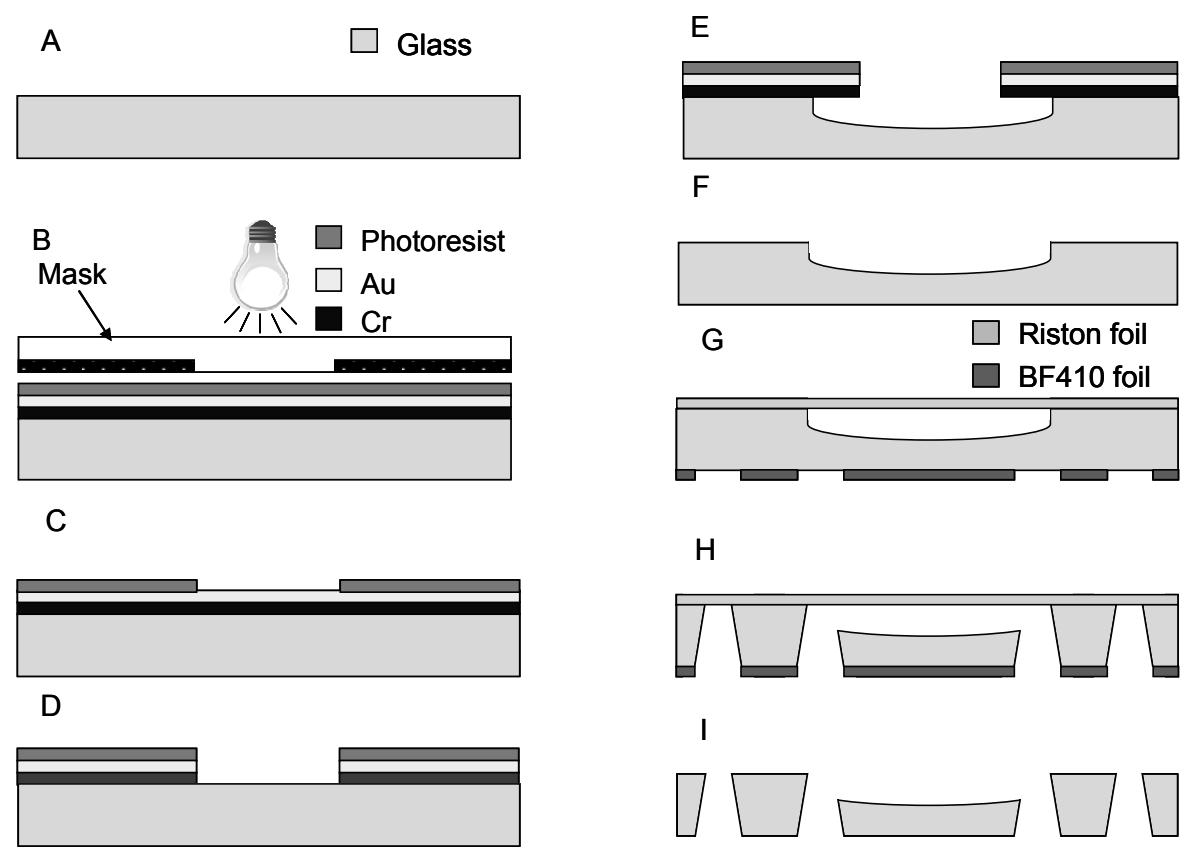

Figure 7.3. Process flow for the fabrication of the microfluidic structures and reservoirs in the glass wafers

A) Wafer cleaning in fuming $100 \% \mathrm{HNO}_{3}$. B) Photolithography step to define the microfluidic structures. C) Patterning of the developed photoresist. D) Patterning of the metal layers to give a hard mask for the channel fabrication. E) Wet-etching of the microfluidic structure in the glass wafer with a 4:1 50\% $\mathrm{HF}: 36 \% \mathrm{HCl}$ solution. F) Removal of the photoresist and metal layers. G) Lamination and patterning of the photosensitive and Riston foil. H) Powder-blasting of the access holes. I) Final device after removal of the photosensitive foils.

Following this, reservoirs ( $1 \mathrm{~mm}$ diameter) are fabricated in one of the two glass wafers using powder-blasting techniques. The wafers are firstly baked at $95^{\circ} \mathrm{C}$ for $2 \mathrm{~min}$ to remove any trace of water. Next, a photosensitive foil (BF410, Ordyl) is laminated on the bottom of the glass wafer, patterned with photolithography techniques $\left(15 \mathrm{~s}\right.$ at $\left.12 \mathrm{~mW} / \mathrm{cm}^{2}\right)$ to define the access holes and finally developed by sonicating the wafer for $3 \mathrm{~min}$ at $40^{\circ} \mathrm{C}$ in a $0.2 \%$ sodium carbonate solution (figure 7.3G). As before, the backside of the wafer is covered with a Riston foil to protect the microfluidic structures. The access holes are powder-blasted from the backside of the wafer using $\mathrm{Al}_{2} \mathrm{O}_{3}$ powder (grain size $29 \mu \mathrm{m}$ ) (figure $7.3 \mathrm{H}$ ). The photosensitive foil is removed, once the holes are made, by soaking the wafer in acetone (Fisher Scientific) and cleaning it $15 \mathrm{~min}$ in DI water in an ultrasonic bath (figure 7.3I) to get rid of $\mathrm{Al}_{2} \mathrm{O}_{3}$ powder. Finally, any polymer residues are removed in $100 \% \mathrm{HNO}_{3}$ for $10 \mathrm{~min}$. 


\section{(ii) Silicon shadow mask}

Micrometer-sized apertures and access holes are etched in the Teflon foil with the help of a shadow mask, which is firstly produced from silicon as follows (see figure 7.4). A silicon wafer (CZ-silicon, 4 inch diameter, $525 \mu \mathrm{m}$ thickness, Okmetic, Vantaa, Finland) is cleaned in $100 \% \mathrm{HNO}_{3}$ for $10 \mathrm{~min}$ after a $1 \% \mathrm{HF}$ dip, rinsed in DI water and dried (figure 8.4A). Subsequently, the wafer is patterned using a standard photolithography process (figures 7.4B-C) and 100- $\mu \mathrm{m}$ deep structures defining the aperture and the access holes, are etched using deep reactive ion etching (DRIE) (Adixen SE; standard Bosch process) [27] (figure 7.4D). Following this, the wafer is cleaned for $10 \mathrm{~min}$ in an oxygen plasma (Tepla, barreletcher) and subsequently $10 \mathrm{~min}$ in a piranha solution $\left(2: 1 \mathrm{H}_{2} \mathrm{SO}_{4}: \mathrm{H}_{2} \mathrm{O}_{2}\right)$ at $120^{\circ} \mathrm{C}$ (figure 7.4E). A thin layer of silicon-rich nitride $\left(\mathrm{Si}_{x} \mathrm{~N}_{\mathrm{y}}\right)(\sim 100 \mathrm{~nm}$ thick) is grown on top of the silicon using low-pressure chemical vapor deposition (LPCVD) techniques (Amtech tempress diffusion system) to protect it during the subsequent wet-etching process (figure 7.4F).

The $\mathrm{Si}_{\mathrm{x}} \mathrm{N}_{\mathrm{y}}$ layer is patterned on the backside of the wafer using a reactive ion etching (RIE) process (Elektrotech Twin system Plasmafab (PF ) (figure 7.4G) to give a hard mask for the last wet-etching step where the holes etched by DRIE are opened up from the backside. Thereafter, the silicon wafer is etched on its backside using wet-etching techniques (25\% $\mathrm{KOH}$ at $75^{\circ} \mathrm{C}$ ) until a $50-\mu \mathrm{m}$ thick layer is left. This resulting wafer is rinsed with DI water and dried. The residual silicon-rich nitride on the bottom of the holes is etched by RIE in the same way as described above. This gives the shadow mask employed for patterning the Teflon foil (figure $7.4 \mathrm{H}$ ).

\section{(iii) Teflon sheets}

The Teflon sheets are processed at the wafer level and only cut into chip-sized pieces during the device assembly. In a first step, a Riston foil is stretched on the bottom of a stainless steel ring (outer diameter $15 \mathrm{~cm}$; inner diameter: $12 \mathrm{~cm}$; thickness $1.5 \mathrm{~mm}$ ) (see figure 7.5A) and a round aperture having a diameter slightly larger than that of a wafer (i.e. $>100 \mathrm{~mm}$ ) is cut out in the middle of this foil. Following this, a piece of a Teflon FEP foil (thickness: $12.5 \mu \mathrm{m}$ ) having a size slightly larger than that of a wafer is rolled across this aperture and heated at $95^{\circ} \mathrm{C}$ for $20 \mathrm{~s}$ to bring it under tension. The silicon-based shadow mask is secured on a second stainless steel ring (outer diameter: $12 \mathrm{~cm}$; inner diameter: 94 $\mathrm{cm}$; thickness $1.5 \mathrm{~mm}$ ) (see figure 7.5B), and subsequently placed on the top of the Teflon foil, as close as possible to the Teflon foil (to prevent the formation of an undercut during the etching process). The two rings are assembled, and the Teflon foil is pushed through the aperture in the Riston foil by the shadow mask (see figures 7.5C-D). The apertures and access holes are etched into the Teflon foil using RIE techniques (Elektrotech Twin system Plasmafab (PF) with an etching speed of approximately $300 \mathrm{~nm} / \mathrm{min}$ ) (see figure 7.5D). The etched Teflon FEP foil is left on the ring to facilitate further handling during the assembly process. 


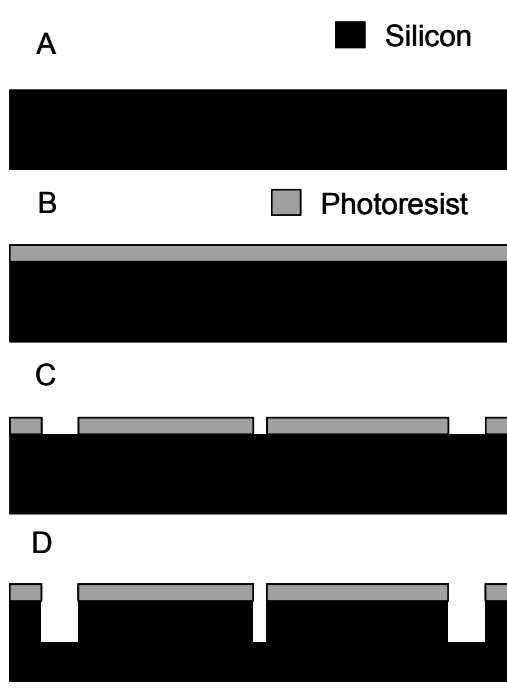

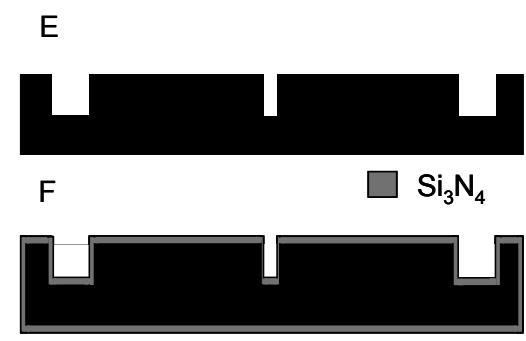

G
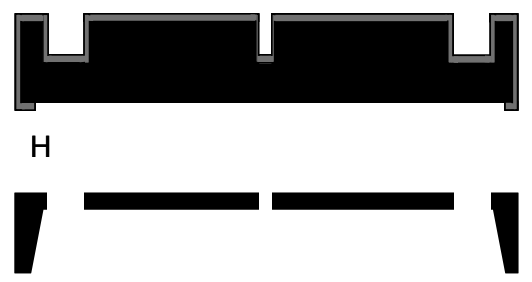

Figure 7.4. Process flow for the fabrication of the silicon-based shadow mask

A) Cleaning of a silicon wafer fuming $100 \% \mathrm{HNO}_{3}$. B) Photolithographic patterning of a 1.7- $\mu$ m photoresist layer deposited to define the microfluidic structures. C) Removal of the photoresist. D) Anisotropic etching of silicon (DRIE techniques, Bosch process). E) Removal of the remaining photoresist. G) Deposition of a Six $N_{y}$ layer (LPCVD). G) Patterning of the backside of the Six $N_{y}$ layer. H) Final device after the thinning of the silicon wafer down to $50 \mu \mathrm{m}$ with the wet-etching technique $\left(25 \% \mathrm{KOH}\right.$ at $\left.75^{\circ}\right)$ and removal of any residual silicon nitride.

\section{(iv) Packaging}

The final devices are produced by covalently bonding the three layers together once they have been aligned. Bonding of the devices is done at the chip level as dicing of the assembled wafers may be hindered by the differences in material properties (glass and Teflon foil). Therefore, the individual glass chips are released prior to the bonding procedure by dicing the wafers into $1 \mathrm{~cm} \times 2 \mathrm{~cm}$ pieces using dedicated equipment (Disco DAD321 blade type TC 300, spindle rev. 25,000 rpm, feed $4 \mathrm{~mm} / \mathrm{s}$ ). As before, the wafers are protected during this dicing step by a Riston foil. The Teflon sheets are cut after the assembly step; this facilitates their manipulation and prevents them from wrinkling. Since no standard process is available to bond Teflon to glass, a technique recently developed in our lab is employed, that relies on the use of an intermediate layer of glue [28]. This approach is flexible; it can easily be adapted as a function of both the nature of the materials to be assembled and the size of the microstructures. Here, NOA- $83 \mathrm{H}$ is employed as gluing material; this resin can be cured thermally (between $60-125^{\circ} \mathrm{C}$ ) or by exposure to UV light $(\lambda=320-380 \mathrm{~nm}$, with a sensitivity peak around $\lambda=365 \mathrm{~nm})$. 

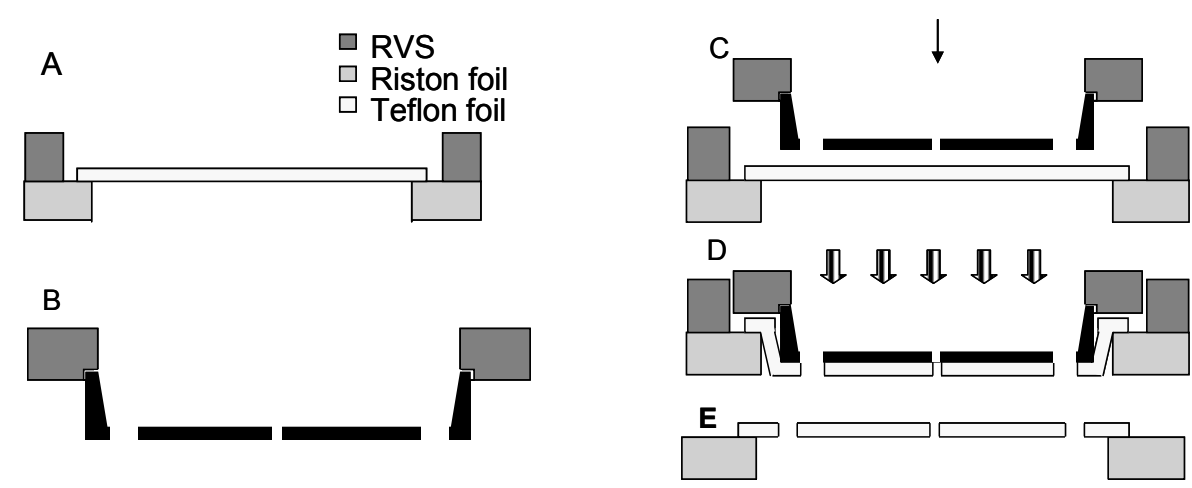

Figure 7.5. Flow-chart for processing of the Teflon foil

A) Stretching of a Teflon foil using a stainless ring and an intermediate Riston foil. B) Insertion of the silicon shadow mask into a second stainless steel holder. C) Assembly of the two holders containing the shadow mask and the Teflon foil. D) Dry-etching of the reservoirs and apertures in the Teflon foil (RIE techniques). E) Disassembly of the two stainless steel holders.

Prior to bonding, the two glass substrates are cleaned in a freshly prepared piranha solution $\left(3: 1 \mathrm{H}_{2} \mathrm{SO}_{4}: \mathrm{H}_{2} \mathrm{O}_{2}\right)$ for $10 \mathrm{~min}$, rinsed in DI water and dried. The resin NOA-83H is spin-coated on a cleaned glass substrates (Spin 150, APT GmbH Automation und Produktionstechnik, Bienenbüttel, Germany spinner; $5 \mathrm{~s}$ at $500 \mathrm{rpm}$ followed by $30 \mathrm{~s}$ at 3500 $\mathrm{rpm}$ ). The resulting gluing layer is briefly (50 s) exposed from the backside to UV light (366 nm; $1 \mathrm{~mW}$; NU-6KL, Konrad Benda Laborgeräte, Wiesloch, Germany) to only leave the part of the resin in contact with oxygen uncured. This uncured glue is subsequently transferred with a roller onto the glass substrates to give a thin layer of gluing material on them (see figure 7.6A). The Teflon foil is carefully aligned under a microscope on the bottom glass substrate with the help of the alignment marks. Thereafter, the Teflon foil is cut using a sharp knife around the edge of the glass substrate, and the top glass substrate is aligned in the same manner on it (see figure 7.6B). Finally, the assembled device is placed for at least 1 hour in an oven at $125^{\circ} \mathrm{C}$ to completely cure the NOA- $83 \mathrm{H}$ resin and bond thereby the three layers together (see figure 7.6C).

For recycling of the devices, the complete devices are immersed in dichloromethane (Sigma Aldrich) for at least a week to dissolve the glue and release the three layers. The Teflon sheet is discarded while the two glass substrates are thoroughly rinsed with acetone (Fisher Scientific) and cleaned in piranha. After the cleaning procedure, the glass parts can be used again. 
B
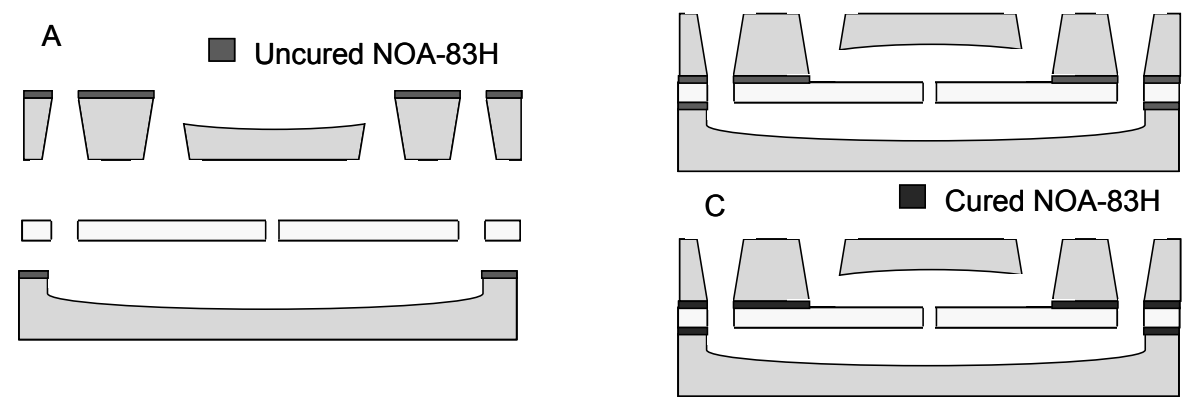

Figure 7. 6. Bonding process

A) Application of a thin layer of gluing material (NOA-83H) on both glass substrates (see [28]) B) Alignment of the Teflon foil (manually) successively with the bottom and the top glass substrates and assembly of the three-layer device. C) Final device after the curing of the gluing material (> 1 hour at $125^{\circ} \mathrm{C}$ ).

\section{Measurement set-up}

\section{(i) Chip holder}

For the fluidic and electrical connections to the device, a home-made developed chip holder is used (see figure 7.7). It consists of two pieces between which the chip is inserted and that are screwed together for tight fluidic connections. The black anodized aluminum bottom part includes a cavity where the chip is inserted; this cavity has the same dimensions and contains a machined rectangular aperture in its middle for microscopy-based characterization. The top part consists of a thick block of black Delrin ${ }^{\circledR}$ in which two series of holes have been machined (i) for inserting assembly screws and (ii) for the electric and fluidic connections to the chip reservoirs, respectively. Finally, a piece of Viton (DuPont Performance Elastomers, Wilmington, Delaware) that also includes an optical window is placed at the bottom of the cavity as a damping layer between the chip and its holder. The chip holder is placed on the microscope stage in a dedicated plate also fabricated in-house.

\section{(ii) Electrical measurements}

The chip is firstly placed in its dedicated holder. The two microfluidic channels are filled with a buffer solution $(1 \mathrm{M} \mathrm{KCl}, 10 \mathrm{mM}$ Hepes, $\mathrm{pH} 7.4)$ introduced from the access holes of the chip-holder. Subsequently, home-made $\mathrm{Ag} / \mathrm{AgCl}$ electrodes are introduced in reservoirs 1 and 2 of the chip (see figure 7.8) so that the each side of the membranes is connected to one electrode. Electrical measurements are carried out with an Axopatch 200b amplifier (Molecular devices, Sunnyvale, CA), applying voltages and measuring currents across the bilayer. Data are acquired using a LabVIEW interface and a PCI-6259 data acquisition card (National Instruments, Austin, TX). All measurements are performed at room temperature. 

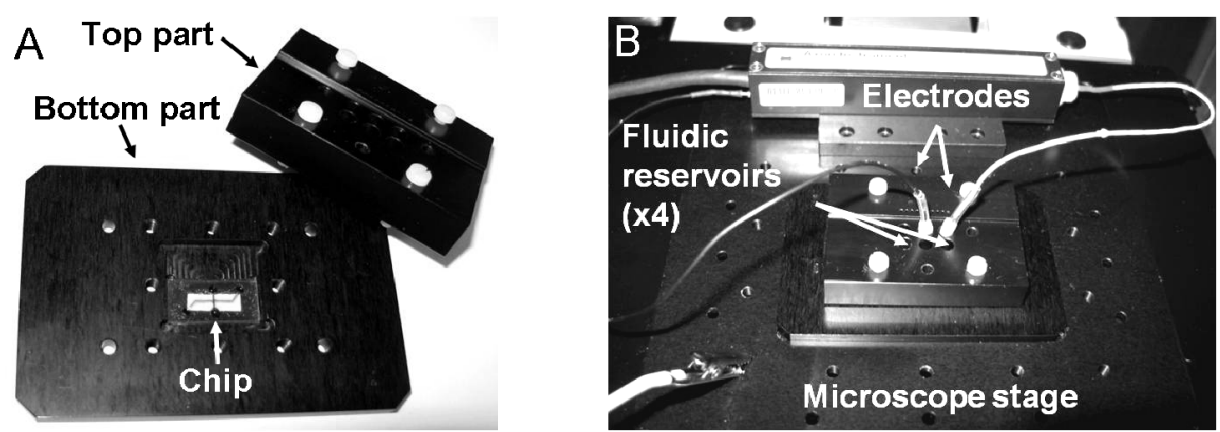

Figure 7. 7. Chip holder

Photos of the in-house developed chip holder for facilitating chip manipulation and fluidic and electric connections. A) Open chip holder showing the different parts of it as well as the chip inserted therein. The bottom plate contains a cavity with an optical window for optical measurements in which a Viton foil (not visible on the picture) is placed as a damping layer before the microfluidic chip is introduced (the channels filled with an ink for their visualization). The top part of the chip holder includes the access holes and four plastic screws for its tight assembly with the bottom part. B) Assembled chip holder placed on the stage of the microscope. Four of the six access holes in the chip holder are used for the fluidic connections while only two are employed for the electrical connections. For that purpose, two $\mathrm{Ag} / \mathrm{AgCl}$ electrodes which are connected to the patch-clamp amplifier are introduced in the reservoirs.

\section{(iii) Optical measurements}

The chip holder in which the microfluidic device has been inserted is placed on the stage of an inverted microscope (Leica DMI 5000M) for optical measurements using both bright field or fluorescence microscopes (N2.1 and blue-green-red (BGR) filter, respectively). Optical recording employs a camera (Leica DFC 310 FX) attached to the microscope and connected to a computer.

\section{Preparation and characterization of BLMs}

\section{(i) Chemicals}

All lipids are used as chloroform-based solutions $(10 \mathrm{mg} / \mathrm{mL}$ phospholipid in chloroform). Before the preparation of the BLMs, a few tenths of $\mu \mathrm{L}$ of the phospholipid solution (DPhPC $+1 \%$ weight DOPE-rhodamine $\mathrm{B}$ ) in chloroform is left to evaporate overnight, yielding an amount of $250 \mu \mathrm{g}$ dried lipids. This dried lipid film is subsequently dissolved in n-decane at a final concentration of $25 \mathrm{mg} / \mathrm{mL}$. The resulting $\mathrm{n}$-decane solution is used for the preparation of BLMs. 


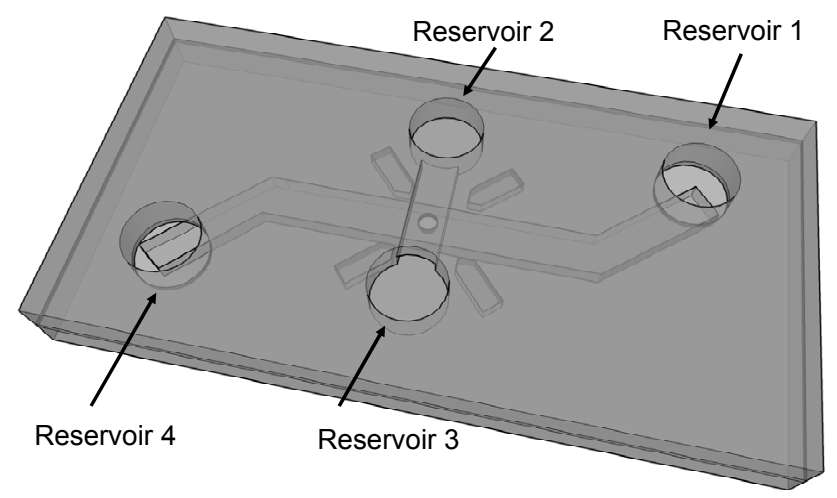

Figure 7.8. Assembled BLM microdevice.

Schematic drawing showing the assembled microfluidic device for BLM experimentation. The aperture is located at the channel intersection at the middle of the alignment marks. The four reservoirs connecting to either the top (2, $3)$ or the bottom $(1,4)$ channel are employed for fluidic handling and electric measurements (after manual insertion of electrodes in the reservoir of the device).

\section{(ii) BLM formation}

Bilayer lipid membranes in the fully microfluidic system are created using a novel methodology (Lipid Plug Thinning (LPT) method), inspired by the works of Suzuki et al. [33] and Sandison et al. [26] and that, as is suggested by its name, relies on the thinning of a lipid plug introduced in a micrometer-sized aperture into a BLM. This novel methodology differs from the other techniques in that thinning of the phospholipid plug occurs spontaneously upon flushing buffer solution in the microchannels, and not by exposing it to a pressure (hydrostatic pressure or air pressure) (see figure 7.9). Firstly, the microchannels are filled with the phospholipid solution (DPhPC $+1 \%$ weight DOPE-rhodamine B in ndecane) introduced in reservoirs 1 and 2 (figures 7.8 and $7.9 \mathrm{~B}$ ) using a syringe and manually applied pressure. Following this, buffer solution $(1 \mathrm{M} \mathrm{KCl}, 10 \mathrm{mM}$ Hepes, $\mathrm{pH} 7.4)$ is slowly introduced in the channels from reservoirs 1 and 2 (figures 7.8 and 7.9C) to leave a thick plug of phospholipids in the micrometer-sized aperture in the Teflon foil (figure 7.9D). The thinning process initiates automatically (figure 7.9E) to give a phospholipid bilayer across the aperture (figure 7.9F). When no lipid plug remains in the aperture or when the thinning process is not successful (i.e. if the plug has not started to thin after $\sim 5$ minutes), the process is started anew. The phospholipid solution remaining in reservoirs 3 and 4 (see figure 7.8) is flushed back into both the top and bottom channels followed by buffer solution until a BLM is successfully obtained in the aperture (figures 7.9G-I).

The formation of a bilayer structure from the phospholipid plug is monitored both optically and electrically. Firstly, a BLM is optically characterized by the observation of a dark area (BLM) surrounded by a fluorescent ring (annulus); the surface area of the dark region corresponds to the surface area of the bilayer. Secondly, the formation of a phospholipid plug into the aperture results in the almost total disappearance of the leakage 
current, indicating the formation of a tight seal between the phospholipid solution and the substrate. However, the thickness of the phospholipid plug cannot be derived from the seal resistance. The value of the residual current is only used to determine the sealing quality. When a tight seal is formed, the membrane capacitance is subsequently measured as previously described [29] both to confirm the formation of a BLM as well as to estimate the surface area of the membrane using equation 7.1 :

$$
A=\frac{C_{\text {measurued }}}{C_{\text {specific }}}
$$

where $A$ stands for the membrane surface area in $\mathrm{cm}^{2}, C_{\text {meas }}$ for the measured capacitance in $\mu \mathrm{F}$, and $C_{\text {specific }}=0.45 \pm 0.05 \mu \mathrm{F} / \mathrm{cm}^{2}$ (the specific capacitance per surface area for a phospholipid bilayer)[30]. This value is compared to the surface area of the dark region determined optically.
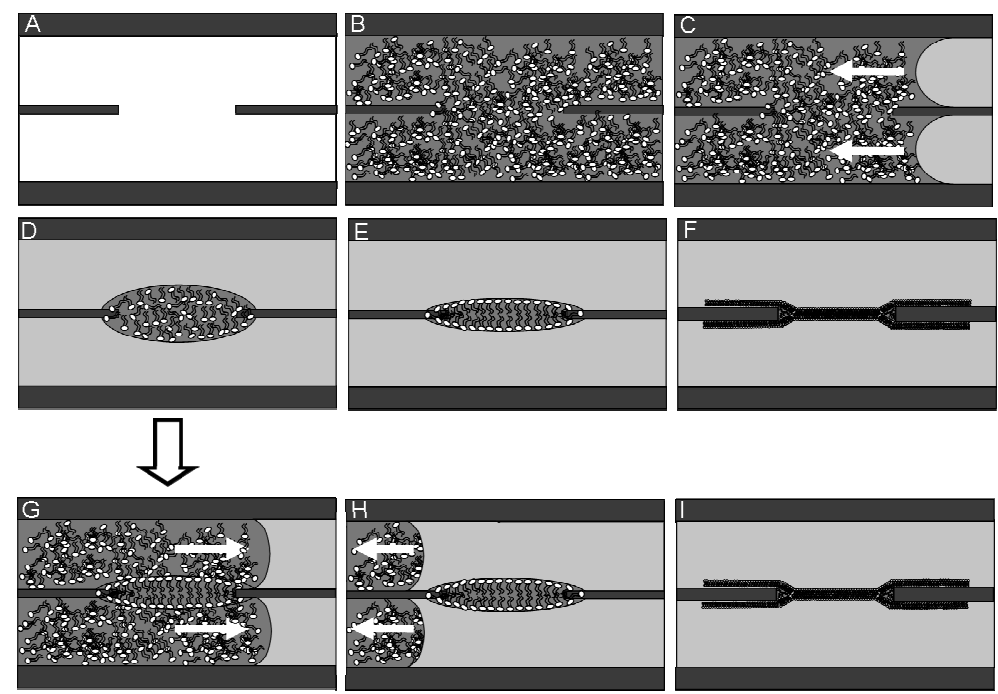

Figure 7.9. BLM formation.

A) BLM formation device prior to membrane preparation: channels are empty. B) Introduction of the phospholipid solution. C) Flushing of the channels with buffer solution ( $1 \mathrm{M} \mathrm{KCl}, 10 \mathrm{mM}$ Hepes, $p H$ 7.4). D-F) Formation of a phospholipid plug in the aperture which spontaneously thins into a bilayer. G-I) Repetition of the whole process in case of unsuccessful bilayer formation.

\section{(iii) Protein insertion}

Alternatively, the formation of a bilayer is confirmed with the addition of membrane proteins therein, since the latter only insert in bilayer and not in multilayer structures. A commonly used protein is the ion channel $\alpha$-hemolysin ( $\alpha$-HL). $0.1 \mu \mathrm{L}$ of an $\alpha$-hemolysin solution ( $2 \mathrm{mg} / \mathrm{mL}$ in DI water) is added and flushed in the top channel from reservoir 2 (ciscompartment). Proteins insert spontaneously in the bilayer. Individual insertions are 
monitored electrically by recording the current across the membrane: every insertion gives rise to a characteristic jump in the current since $\alpha$-hemolysin has a conductivity of $1 \mathrm{nS}$ [18] (i.e. $50 \mathrm{pA}$ increase in the current when a $50 \mathrm{mV}$ voltage is applied for a buffer solution of $1 \mathrm{M} \mathrm{KCl})$.

\section{Results and discussion}

In the coming section, firstly the outcome and reproducibility of the fabrication process are described. Following, the successful BLM formation is demonstrated in the novel device for membrane studies.

\section{A. Chip design and fabrication}

\section{(i) Chip design}

The layout of the microfluidic device is chosen to fit basic experimental wishes and to be possibly upgraded with minor modifications. The two fluidic "reservoirs" must be independent, placed on both sides of a micrometer-sized aperture and possibly scaled down in the future for improved electrical measurements and faster solution refreshment on both sides of the membranes. On other aspects, the BLMs are formed between the two reservoirs and are to be characterized optically, so that the reservoirs must be placed horizontally. Consequently, the microfluidic device consists of a 3-layer structure as shown in figure 7.2. It firstly includes two layers of microfluidic channels to form the trans- and cis-compartments, and their dimensions can easily be decreased for further miniaturization of the fluidic reservoirs. The third layer is a substrate placed between the two fluidic layers and in which a micrometer-sized aperture is machined. Finally, the two cis- and trans-microchannels are arranged in a perpendicular configuration, with the aperture located at their intersection; this layout was chosen as it can easily be upgraded for the production of large arrays of membranes for multiplexed studies, in the future.

\section{(ii) Material choice}

In the same way as for the layout of the device, the materials to produce the microfluidic devices are chosen to meet the requirements of the future experimentation while they are easily machined using microfabrication techniques. The final devices must be suitable for both optical (bright field and fluorescence) and electrical measurements on the BLMs so that the materials must (i) be transparent in the visible spectrum, (ii) give no background fluorescence and (iii) be electrically insulating. On other aspects, the device should preferably combined both hydrophobic and hydrophilic materials. Specifically, the microfluidic channels are machined in a hydrophilic material for easy fluid handling and to prevent the phospholipids from absorbing on the channel walls. For the third layer a hydrophobic substrate is employed, as is done for most BLM experimentation devices: the BLM is formed across the substrate with their hydrophobic tails sitting on the substrate. This tight bind between the phospholipids and the substrate is a guarantee for the formation of the $G \Omega$ seal required for sensitive electrical measurements. 
With these requirements, glass is chosen as a substrate to fabricate the microchannels. Fabrication of microchannels in glass is a standard routine using microtechnology techniques, and this material is electrically insulating and transparent, making thereby dual sensitive electrical measurements and high-quality optical characterization possible. On other aspects, glass is inert to a large amount of solvents and chemicals, which is not the case of most polymer-based materials, and it can easily be cleaned for recycling of the microdevice. For the aperture-containing substrate, Teflon, which is the most common substrate for BLM experimentation, is retained in the form of a thin (12.5 $\mu \mathrm{m}$ thickness) commercially available FEP (Fluorinated ethylene propylene) foil. This inert and hydrophobic material is like glass, electrically insulating and transparent, which suppresses issues associated with stray capacitances and leakage currents.

\section{(iii) Fabrication process}

The fabrication process of the complete device includes three aspects: the fabrication of the microchannels in the glass substrate, the production of the aperture-containing membrane and the assembly of the three layers to give the final device. The first aspect, i.e. the wet-etching of microchannels in glass substrates is a standard process in microtechnology. However, the two other aspects had to be developed in this work and are worth discussing.

The second step in the fabrication is the production of structures (micrometer-sized aperture and access holes) in the intermediate Teflon foil. Different processes are available for this: laser-micromachining, mechanical drilling, and dry etching techniques. Lasermicromachining techniques are becoming highly popular for structuring polymer-based materials. However, the cleanliness of the resulting structures highly depends on their size and the characteristics of the laser employed, and this often results in the creation of debris around the fabricated structures. On other aspects, these techniques are not easily accessible in our laboratory. Similarly, mechanical drilling is limited to large structures and is not applicable anymore for the production of smaller apertures in the future. Therefore, dry etching techniques, which are routinely employed in our clean-room facilities, are used here. Production of the apertures requires the development of a new process making use of a shadow mask for the etching step, as conventional masking layers produced by photolithography techniques are precluded on such a thin substrate. This fabrication process is going to be published soon.

Teflon FEP foils (12.5 $\mu \mathrm{m}$ thickness) are employed and patterned using DRIE techniques with the help of a shadow mask based on silicon. Apertures sizes with a diameter of 10, 50 and $100 \mu \mathrm{m}$ are included in the same mask. All sizes drawn in the mask resulted in circular apertures, however, due to under-etching their actual size in the Teflon FEP foil was approximately $10 \%$ larger. For instance, when a $50 \mu \mathrm{m}$ diameter hole in the mask is employed, the resulting apertures in the Teflon FEP foil have a diameter of $54.7 \pm 3.2$ $\mu \mathrm{m}$. The fabrication of the apertures is highly reproducible; the apertures are well-defined, 
circular and "clean" for the different sizes tested here. Figure 7.10 shows one aperture of $56.6 \mu \mathrm{m}$ diameter produced in a Teflon FEP foil.

A main limitation in this technique is the fabrication throughput as chips are produced one at a time from a large piece of Teflon foil, which is unusual for microfabrication processes. This makes the whole fabrication process even more onerous as the Teflon foil is discarded after disassembly of the device while only the glass substrates are recycled and used again. On other aspects, the size of the aperture cannot be scaled down below $\sim 10 \mu \mathrm{m}$ for these FEP foils of $12.5 \mu \mathrm{m}$ thickness because of the limited thickness of the shadow mask. Finally, the thickness of the Teflon foil cannot be varied. On one hand, a thicker Teflon foil gives irreproducible results in terms of etching as prolonged heating of the thicker foils during the etching process also results in wrinkling of the material. On the other hand, thinner foils would be even more difficult to handle without extensive wrinkling, as discussed later. Ideally, the intermediate Teflon foil should also be processed at the wafer scale like the glass substrates, its thickness should be possibly varied and the size of the apertures decreased. For these reasons, we have recently started to work on another strategy to produce the intermediate substrate containing the micrometer-sized apertures. It consists of spin-coating Liquid Teflon AF (type 1601, 6\% in Fluorinert FC-40, DuPont Fluoroproducts, Wilmington, Delaware, USA) on a structured PDMS-based (Polydimethylsiloxane) substrate. Upon evaporation of the solvent in the Teflon, a solid Teflon foil is obtained that contains the same structures as the PDMS layer [31]. Thereby, (i) the size of the structures is easily changeable; and (ii) the thickness of the foil can be varied by changing the parameters for the spin-coating step.

The last aspect is the assembly of the three layers to form the final devices. Two routes are conceivable for this: covalent bonding of the three layers or their clamping using clips or a chip holder. Yet, the alignment of the three layers is crucial so that the aperture is located at the channel intersection and with the second option, this alignment may easily be lost upon manipulation of the microchip. Consequently, we decided to bond covalently the three layers of glass and Teflon. However, there is no standard technique available to bond Teflon and glass substrates although a couple of strategies have been reported in the literature [3234]. For this, we decided to apply a new bonding route recently developed in our group that relies on the use of an intermediate layer of glue [28]. With this technique, a very thin layer of adhesive is dispensed on one of the substrates to be bonded, which minimizes the risk of clogging of the structures upon entry of the glue in the channels by capillary action. This aspect is especially important when working with narrow or shallow structures, which are prone to fill quickly with the glue. The microchannels in the current design are still relatively large, but they will be downscaled in the future, and the aperture(s) could also clog with the glue. On other aspects, this technique is highly versatile as it can be derived for a large variety of gluing materials presenting different physical and chemical properties and that can be cured under different conditions (temperature vs. UV irradiation, at different temperatures or using various wavelengths, for instance). We chose here to work with the 
commercially available optical adhesive NOA-83H; it adheres on glass and Teflon (which is not the case of most of the adhesives), and it can be cured both thermally $\left(125^{\circ} \mathrm{C}\right)$ and optically (UV irradiation, $\lambda=366 \mathrm{~nm}$ ). Lastly, this adhesive can be chemically dissolved, which makes the glass parts reusable, reducing the fabrication costs of the device.

A tricky aspect in the bonding step is the positioning of the Teflon foil between the two glass substrates; this includes not only the alignment of the aperture at the intersection of the microchannels but also proper straightening of the Teflon foil. As the foil is very thin, it tends to wrinkle, and this prevents proper and leakage-free bonding of the device. Another option would be to employ thicker Teflon foil $(25 \mu \mathrm{m})$, but as mentioned, the latter gave irreproducible and unreliable results for the etching of the aperture using DRIE techniques. As the Teflon is mounted and stretched on a stainless steel ring for the etching step, it is directly used on the ring for alignment and bonding. Alignment of the three layers is monitored with the help of four dedicated marks etched in a glass substrate and that should be placed around the micrometer-sized aperture (see figure 7.10), and with the position of the reservoirs; this is done manually at the chip level under a microscope. The aperture is typically aligned with up to $35 \mu \mathrm{m}$ offset from the center of the channels, which does not cause any problems for the functionality of the device since the channel structures are much bigger.

\section{B. BLM experimentation}

\section{(i) BLM formation and characterization}

BLMs are formed in the devices with a technique developed in-house (the LPT method) that borrows much from other techniques reported in the literature [26]. Yet, thinning of the lipid plug in the aperture relies here on flushing a buffer solution and not on exposing the membrane to a pressure, as reported by other groups (see figures 7.9 and 7.11). With this technique, the bilayer formation is very reproducible; $75 \%$ of the attempts result in the creation of a stable membrane the first time, while for the $25 \%$ other attempts, a second flushing cycle is required before a stable membrane is obtained. Furthermore, the resulting membranes are very stable, and exhibit a lifetime of up to 7 hours, which is comparable to similar miniaturized systems $[5,26]$. The success of membrane formation mainly depends on the speed at which buffer is added in the channels to flush the phospholipid solution. When buffer is slowly introduced, the success rate for membrane formation is higher than when the buffer is too quickly flushed. As for most other techniques, the creation of a membrane becomes much easier from the second cycle, either after breaking the membrane or as a second attempt. Another advantage is that only a few $\mu \mathrm{L}$ of the lipid solution of the buffer is required to fill in the device. 

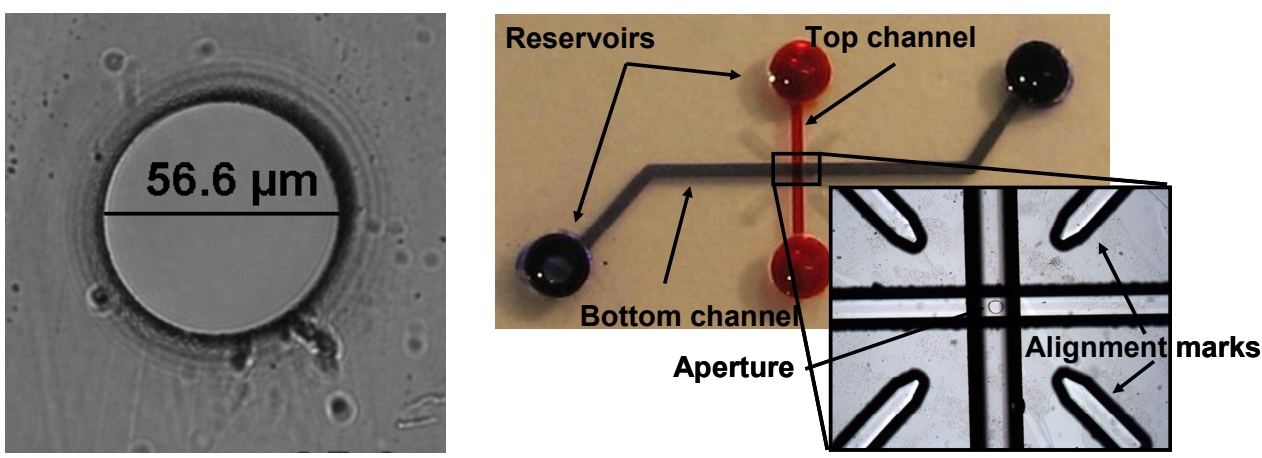

Figure 7. 10. Microfabicated aperture and assembled BLM microchip.

Left: Aperture (56.6 $\mu \mathrm{m}$ in diameter) formed with RIE techniques in a $12.5 \mu \mathrm{m}$ thick FEP Teflon foil. Right: Picture of the assembled device. The top channel is filled with a red ink and the bottom channel with a black ink to check the absence of leakage and consequently good bonding between the different layers. The inset shows the intersection of the channels with the micrometer-sized aperture $(100 \mu \mathrm{m})$ in the middle and the alignment marks etched in a glass substrate.

The resulting BLM almost spans the entire aperture (see figure 7.11F). Approximately $85 \%$ of the total surface area is taken up by the bilayer, whereas the remainder $(\sim 15 \%)$ is formed by the annulus. This value is good compared to other non-solvent free membrane preparation techniques. For instance, the painting technique in the conventional devices routinely gives BLMs that occupy only half of the aperture and Sandison et al. report 70 $80 \%$ coverage of the aperture with their BLMs using the air-exposure technique in a miniaturized device [26]. However, the membranes obtained with the LPT methodology are not solvent free, which means that an annulus is always present. The development of a novel solvent-free technique would probably help increasing the surface area of the bilayer structure even further.

Three aspects are important in the method employed to prepare the BLMs. These are the reproducibility of the membrane, the possible automation of the process of membrane formation and the success yield.

The approach employed here for the preparation of the membranes (LPT method) is comparable to other works reported in the literature from the groups of Takeuchi $[5,19,35-$ 37] and Morgan [26, 38], in that they proceed in the same way. Firstly, a small amount of phospholipids is deposited in the aperture. Following this, the lipid plug is thinned until a bilayer structure is obtained. Still, a number of differences are found between these techniques on the following aspects. First of all, this work focuses on microfluidic systems while a number of other reports employ half-microfluidic devices [5, 26, 36, 38-39]. Secondly, the phospholipids are deposited from a microfluidic channel [19, 35-37] or from the top of the aperture (for half-microfluidic devices) [26]. Using a microfluidic approach gives better control on the deposition of the phospholipids [35], and the process becomes more reproducible. Similarly, the amount of phospholipids deposited is different for different 
techniques, and this mainly depends on the aperture features, and especially on the thickness of the substrate [36]. If the substrate that contains the aperture is thick [36], more phospholipids are deposited, and it becomes more difficult to trigger the thinning step and the creation of a bilayer [35-36]. If the substrate is thinner [5, 19], the deposition of lipids is more reproducible and a higher success yield for membrane formation is reported; the thinning process is much faster and spontaneous. In our work, the phospholipids are introduced using the microchannels, and this would bring higher control on the amount of phospholipids introduced. On other aspects, our device is closer to that developed by Le Pioufle et al. in terms of feature dimensions [5]. Our Teflon substrate is $12.5 \mu \mathrm{m}$ high (against $20 \mu \mathrm{m}$ ); thereby the amount of phospholipids deposited is limited and more reproducible, which is a guarantee for a higher success yield for membrane formation. A third difference is found in the way the thinning of the lipid plug is triggered. Suzuki et al. apply a hydrostatic pressure with a column of buffer placed above the lipid plug [36], Sandison et al [26] expose the membranes to air to promote thinning while Le Pioufle et al. report spontaneous thinning of the lipid plug when buffer is flushed in the microchannel [5]. As our substrate is also very thin, we think we also have spontaneous thinning of the lipid plug upon flushing the buffer. The $25 \%$ failure is most probably to be attributed to too fast flushing of the buffer in the microchannels. Fourthly, when thinning is triggered by applying a pressure, the whole process must be thoroughly monitored for a high yield of membrane formation and the reproducibility of the process. Most of the time, membrane formation is followed optically [26, 36]. However, when thinning occurs spontaneously, like in the work of Le Pioufle et al. and our work, this monitoring is not necessary anymore. This brings us to the fifth main difference, which is the amenability of the methodology to the automation of membrane formation, and this aspect is essential for high-throughput screening applications. Suzuki et al. [36] do not comment on the automation of the process, and BLM formation is always monitored optically. Sandison et al. [36] characterize the thinning step for its possible automation and determine the time required for the plug lipid to become a bilayer ( $80 \%$ of the aperture area). However, their success yield drops from $80 \%$ to $50 \%$ when the process is not monitored anymore on individual membranes. Spontaneous thinning of the lipid plug appears in that sense to be very interesting for automation of the formation of BLMs in aperture arrays as this would only require minor optimization of the flushing process to give a high yield of BLM formation. This has been reported by Le Pioufle et al. for the formation of arrays of BLMs. Similarly, our technique should easily be automated and suitable for the formation of arrays of membranes. If required, automation of the process could also employ monitoring of the electrical properties of the membrane, which is less demanding for the experimenter. When the measured capacitance and sealing resistance are not satisfactory, the membrane formation process can automatically be repeated.

As explained, BLM formation is monitored using optical and electrical techniques. With the former detection techniques, the creation and thinning of a lipid plug in the 
aperture (see figure 7.11) is observed and the surface area of the bilayer with respect to the annulus can be determined, after thinning of the lipid. After addition of the phospholipid solution, the whole channel becomes bright red fluorescent (see figure 7.11A). Replacement of this lipid solution by buffer (or air) (see figure 7.11B) leaves a thick lipid plug in the aperture where the fluorescence is selectively located (see figure 7.11C). However, as soon as the buffer is flushed into both channels, this fluorescent signal in the aperture is progressively reduced to a bright signal at the edge of the aperture (see figure 7.11D), indicating the thinning of the plug into a bilayer structure. The formation of the bilayer, i.e. thinning of the lipid plug, starts in the middle of the aperture and moves outwards in all directions. At the end of the thinning process, the phospholipid bilayer appears as a darker area in the aperture which makes it easily distinguishable both with bright-field and fluorescent microscopy (see figure 7.11). Furthermore, from the bright-field images, the size of the annulus can be determined as well as that of the bilayer. Subsequently, looking at the surface area of the bilayer (i.e. whether a large enough dark area is obtained) reveals whether a flushing step is required or whether a bilayer membrane has successfully been formed. The membrane shown in figure $7.11 \mathrm{~F}$ has for instance a surface area of $1.59 \times 10^{3}$ $\mu \mathrm{m}^{2}$ (diam. $45 \mu \mathrm{m}$ ) while the area of the aperture is $1.96 \times 10^{3} \mu \mathrm{m}^{2}$ (aperture diam. $50 \mu \mathrm{m}$ ); the membrane spans $\sim 81 \%$ of the total surface area.
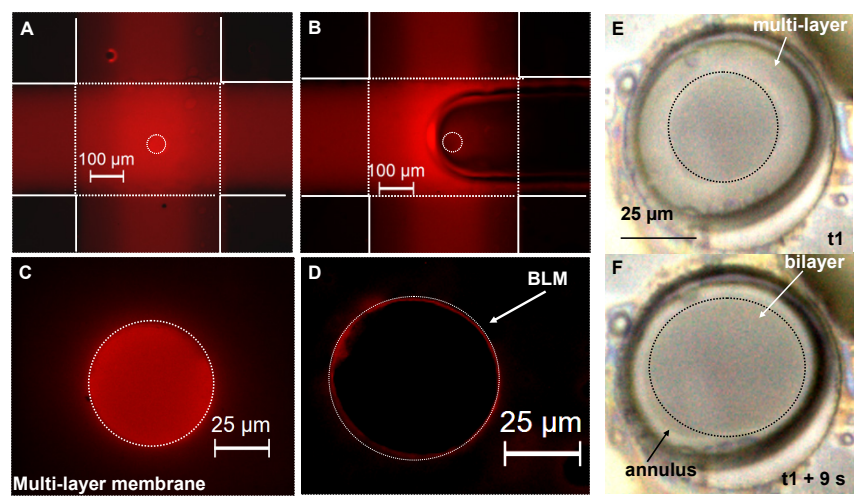

Figure 7.11. Optical detection of BLM formation.

A-D) Fluorescent microscopy photographs of the bilayer formation steps for an aperture of $50 \mu \mathrm{m}$. The borders of the aperture and the channels are indicated with white lines on the pictures. A) In a first step towards membrane formation, the channel is filled with the phospholipid solution (DPhPC $+1 \%$ weight DOPE-rhodamine B in $n$ decane). B) The phospholipid solution is replaced by air followed by buffer solution (1 M KCl, $10 \mathrm{mM} \mathrm{Hepes,} \mathrm{pH}$ 7.4) (the buffer plug is visible in the bottom channel). C) A plug of phospholipids is left behind in the apertures upon buffer insertion and D) forms a bilayer after thinning upon exposure to the buffer flow. E-F) Bright field pictures of the thinning process of the bilayer over a period of $9 \mathrm{~s}$. The BLM area is circled with a black line. The dark bilayer area covers approximately $40 \%$ of the aperture at $t 1$, and grows to represent $80 \%$ of the aperture area $9 \mathrm{~s}$ later. These pictures illustrate that the bilayer formation is a fast process.

The bilayer formation is confirmed electrically both by the measurement of a tight seal 
(high sealing resistance) and a low capacitance. Typically, here, a seal resistance of $14 \pm 4 \mathrm{G} \Omega$ was measured, and a capacitance of $10 \pm 3.8 \mathrm{pF}$ for apertures of $\sim 56 \mu \mathrm{m}$ diameter. The value of the sealing resistance reveals the quality of the seal and the potential existence of a leakage pathway. In the latter case (when the G $\Omega$ seal is not formed), the whole process of BLM formation should be repeated. On other aspects, the value of the capacitance indicates whether a bi- or multilayer structure is obtained and is a measure for the surface area of bilayer membrane. Again, a too low value of the capacitance shows that the bilayer is too thick (multilayer) or the bilayer area very small. In that case, the BLM should be broken and prepared again. From the capacitance values, the BLM surface area can be estimated (using equation 7.1 indicated in section II) to be around the $53 \mu \mathrm{m}$ (aperture size $\sim 56 \mu \mathrm{m}$ ). This indicates that the BLM occupies most of the aperture area $(>80 \%)$ and that the annulus is small compared to the bilayer, as already seen from the optical measurements (see figure 7.11).

\section{(ii) Protein insertions}

A last standard assay to confirm the successful formation of a bilayer structure consists of inserting pore-forming structures (ion channels) therein, as these trans-membrane species only insert in bilayer membranes and not in thicker structures. Additionally, this demonstrates the potentiality of the device for single protein studies which is one of the keyapplications of such platforms. Here, the ion channel $\alpha$-hemolysin is employed as a model for channel protein. $\alpha$-Hemolysin is a water soluble protein formed by Staphylococcus aureus. When it comes into contact with a bilayer lipid membrane it heptamerizes, forming a mushroom like structure with a cap of $\sim 100 \AA$ in diameter and $70 \AA$ in length floating on top of, and a stem ( $\sim 30 \AA$ in diameter, $50 \AA$ long) residing in the bilayer. Alpha-hemolysin contains a channel running through the entire length of the pore, which varies in size along the pore axis. At the entrance of the cap, the pore diameter is $26 \AA$ and reaches a width of 46 $\AA$ in the cap, followed by a shrinkage to $15 \AA$ and finally comes down to a diameter of $20 \AA$ in the stem. $\alpha$-Hemolysin forms stable, non-gated pores in lipid bilayers with approximately ohmic behaviour, although some rectification and a mild anion selectivity are found [17-18].

$\alpha$-Hemolysin is added to the buffer solution in the top channel or cis-compartment $(0.1$ $\mu \mathrm{L}$ of a $2 \mathrm{mg} / \mathrm{mL}$ solution in DI water), and the protein inserts itself spontaneously into the BLM as soon as it touches its surface. The introduction of $\alpha$-hemolysin in the membrane is monitored electrically as each protein insertion gives rise to the creation of a leakage current equal to $50 \mathrm{pA}$ if a $50-\mathrm{mV}$ voltage is applied across the membrane and a buffer solution of $1 \mathrm{M} \mathrm{KCl}$ is employed. Figure 7.12 illustrates this process of protein insertion with several proteins being introduced in the membrane within a several tenths of seconds.

These results clearly show that the microfluidic chip has a potential for single protein studies. Since the insertion of individual protein can be monitored, buffer can be perfused in the chip as soon as one protein is inserted, and measurements on the single protein can be performed. The protein employed in this study is, however, not a relevant model for 
pharmaceutical studies but it can yet be employed for plenty of applications. For that reason, it has notably gained in popularity these last decades where it has been increasingly employed for biophysical studies and polymer analysis, as an alternative to solid-state nanopores. The size of its channel is large enough to let not only ions pass through but also linear polymers such as single stranded DNA (ssDNA) or polyethylene glycol (PEG). Consequently, $\alpha$-hemolysin has been applied for DNA sequencing, fundamental studies on DNA molecules or for polymer separation. Lastly, $\alpha$-hemolysin is often employed as a proofof-principle channel protein to illustrate the feasibility of (single) protein studies in miniaturized and microfluidic devices [8, 15, 37, 40-42] and to confirm the formation of a bilayer structure. Its high stability and easy insertion in membranes make it an ideal entity also to assess the device sensitivity and characterize electrical measurements.

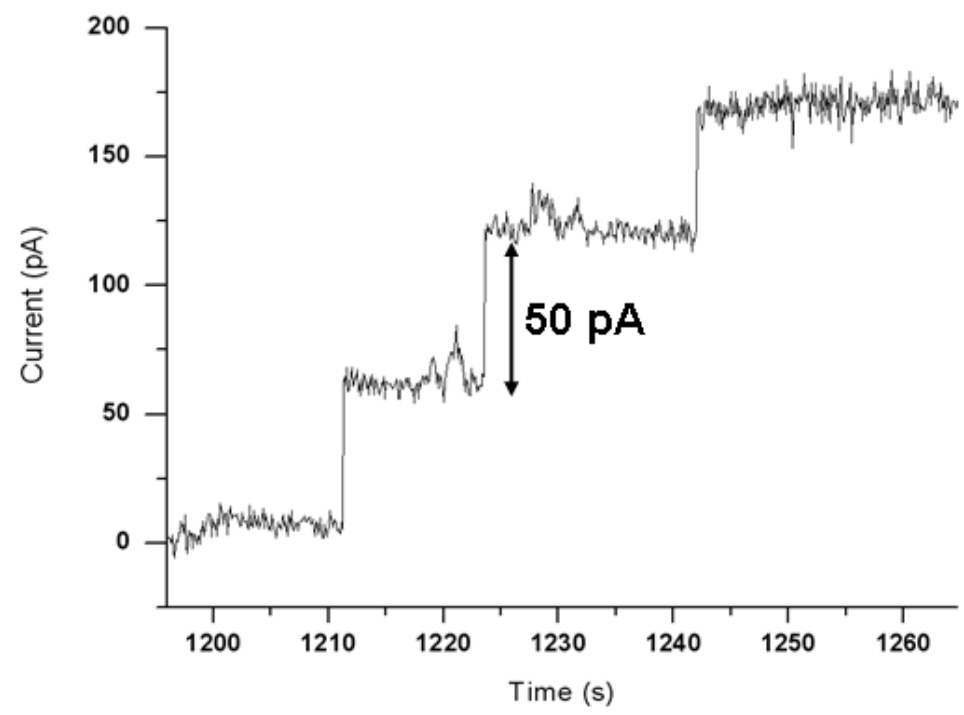

Figure 7.12. Single protein studies.

Electrical monitoring of the insertion of individual $\alpha$-hemolysin protein channels (buffer: $1 \mathrm{M} \mathrm{KCl}, 10 \mathrm{mM}$ Hepes, $p H$ 7.4). Each jump in the recorded current of $50 \mathrm{pA}$ (when $50 \mathrm{mV}$ is applied) corresponds to the insertion of a single protein.

\section{Conclusion and outlook}

We have successfully developed a novel microfluidic device for BLM experimentation and their characterization using a combination of optical (microscopy) and electrical techniques. It consists of a hybrid system made from two glass substrates and a thin Teflon foil placed in between, which includes a micrometer-sized aperture for BLM formation. The structures in glass are fabricated with standard wet-etching techniques, while for the production of the aperture a novel process had to be developed that employs dry-etching 
techniques. Apertures down to $10 \mu \mathrm{m}$ are successfully produced, with a good reproducibility. Finally, three substrates are covalently assembled using intermediate layers of gluing material to yield strongly bonded systems. For BLM experimentation, a new methodology is developed to create BLMs in a closed microfluidic environment. This novel methodology relies on the successive flushing of a lipid plug and buffer solution through the microfluidic channels and across the apertures until a proper BLM is detected using either electrical or optical measurements. With this method, a high success rate for BLM formation is obtained, which is comparable to values reported by other groups in miniaturized devices and the membranes are very stable with a lifetime of up to 7 hours. The device is finally applied for early single protein studies using the protein model $\alpha$ hemolysin.

The design of this device is relatively simple and is limited to single BLM experimentations. However, the design and experimental approach proposed here are suitable for straightforward upgrade towards multiplexed measurements. Firstly, the layout of the microfluidic network can easily be extended to arrays of channels having multiple crossings, allowing for parallelized and independent bilayer assays. Secondly, the fabrication approach employed here to produce the apertures is compatible with the production of arrays of apertures. Another crucial aspect towards multiplexed analysis is the automation of the process of pore formation. In such a multiplexed device, membrane formation should be automated. The technique developed in this work lends itself well to this, and membrane formation can be monitored electrically, and not only optically. The microfluidic device has so far only been tested for $\alpha$-hemolysin studies as proof-of-principle experiments. However, microfluidic platforms, particularly when they include multiple experimentation sites, can find numerous applications: high-throughput screening of drugs for the pharmaceutical industry but also screening of different experimental parameters (membrane composition, protein concentration, buffer composition, exposure to different chemicals) on a series of membranes simultaneously, for more fundamental studies. Similarly, measurements have been restricted to purely electrical techniques using proteins, and optical techniques have only been employed for characterization of the BLM formation. Yet, optical measurements are also possible, although they may require the use of a thin glass as bottom substrate for high-resolution optical measurements. Acknowledgements

I would like to thank Verena Stimberg who has developed the microfluidic system during her master. Hans de Boer, Johan Bomer and Eddy de Weerd are gratefully acknowledged their technical support.

\section{References}

[1] Mueller, P. et al. (1963). J. Phys. Chem 67 (2), pp. 534-535.

[2] Tamm, L.K. \& Mcconnell, H.M. (1985). Biophys. J. 47 (1), pp. 105-113.

[3] Tien, H.T. \& Ottova, A.L. (2001). J. Membr. Sci. 189 (1), pp. 83-117. 
[4] Eray, M. et al. (1994). Biosens. Bioelectron. 9 (4-5), pp. 343-351.

[5] Le Pioufle, B. et al. (2008). Anal. Chem. 80 (1), pp. 328-332.

[6] Fertig, N. et al. (2000). Appl. Phys. Lett. 77 (8), pp. 1218-1220.

[7] McGeoch, J.E.M. et al. (2000). Med Biol Eng Comput 38 (1), pp. 113-119.

[8] Peterman, M.C. et al. (2002). Biomed. Microdevices 4 (3), pp. 231-236.

[9] Baaken, G. et al. (2008). Lab Chip 8 (6), pp. 938-944.

[10] de Almeida, R.F.M. et al. (2003). Biophys. J. 85 (4), pp. 2406-2416.

[11] Feigenson, G.W. \& Buboltz, J.T. (2001). Biophys. J. 80 (6), pp. 2775-2788.

[12] Veatch, S.L. \& Keller, S.L. (2005). Phys. Rev. Lett. 94 (14).

[13] Veatch, S.L. et al. (2004). Biophys. J. 86 (5), pp. 2910-2922.

[14] Dudia, A. et al. (2008). Nano Lett. 8 (4), pp. 1105-10.

[15] Hemmler, R. et al. (2005). Biophys. J. 88 (6), pp. 4000-4007.

[16] van Rooijen, B.D. et al. (2009). BBA 1788 (6), pp. 1271-8.

[17] Korchev, Y.E. et al. (1995). J. Membr. Biol. 143 (2), pp. 143-151.

[18] Menestrina, G. (1986). J. Membr. Biol. 90 (2), pp. 177-190.

[19] Suzuki, H. et al. (2009). Biomed. Microdevices 11 (1), pp. 17-22.

[20] Zagnoni, M. et al. (2009). Anal. Bioanal. Chem. 393 (6-7), pp. 1601-1605.

[21] Zagnoni, M. et al. (2009). Biosens. Bioelectron. 24 (5), pp. 1235-1240.

[22] Whitesides, G.M. (2006). Nature 442 (7101), pp. 368-373.

[23] Keenan, T.M. \& Folch, A. (2008). Lab Chip 8 (1), pp. 34-57.

[24] Holden, M.A. et al. (2003). Sensor. Actuat. B-Chem 92 (1-2), pp. 199-207.

[25] Jeon, N.L. et al. (2000). Langmuir 16 (22), pp. 8311-8316.

[26] Sandison, M.E. et al. (2007). Langmuir 23 (15), pp. 8277-8284.

[27] Jansen, H.V. et al. (2009). J. Micromech. Microeng. 19 (3).

[28] Arayanarakool, R. et al. (2010). Lab Chip 10 (16), pp. 2115-2121.

[29] Wiese, A. \& Seydel, U. (2000). In Methods in Molecular Biology, pp 355-370.

[30] Requena, J. \& Haydon, D.A. (1975). P Roy Soc Lond a Mat 347 (1649), pp. 161-177.

[31] Mayer, M. et al. (2003). Biophys. J. 85 (4), pp. 2684-2695.

[32] Bart, J. et al. (2009). Lab Chip 9 (24), pp. 3481-3488.

[33] Grover, W.H. et al. (2008). Lab Chip 8 (6), pp. 913-918.

[34] Willis, P.A. et al. (2007). Lab Chip 7 (11), pp. 1469-1474.

[35] Suzuki, H. et al. (2004). Lab Chip 4 (5), pp. 502-505.

[36] Suzuki, H. et al. (2006). Langmuir 22 (4), pp. 1937-1942.

[37] Suzuki, H. et al. (2007). Biosens. Bioelectron. 22 (6), pp. 1111-1115.

[38] Sandison, M.E. \& Morgan, H. (2005). J. Micromech. Microeng. 15 (7), pp. S139-S144.

[39] Sandison, M.E. et al. (2007). J. Micromech. Microeng. 17 (7), pp. S189-S196.

[40] Ervin, E.N. et al. (2008). Anal. Chem. 80 (6), pp. 2069-2076.

[41] Mach, T. et al. (2008). Anal. Bioanal. Chem. 390 (3), pp. 841-846.

[42] Osaki, T. et al. (2009). Anal. Chem. 81 (24), pp. 9866-9870. 


\section{Chapter 8}

\section{Miniaturized device for the electroporation of adherent (and polarized) cells}

This chapter focuses on the use of a miniaturized system for cell electroporation. The simple device we propose has been designed for the specific electroporation of adherent MDCK cells, to validate if one of the conclusions from chapter 6, i.e. that the apical membrane is more resistant to electroporation than the basolateral one, still applies in whole cells. In this simple and straightforward system, MDCK cells are cultured under two conditions, to induce or not their polarization, and their sensitivity to electroporation is compared in both cases. 


\section{Introduction}

In this chapter, a miniaturized electroporation device is developed for specific electroporation of monolayers of adherent cells. A specific application targeted here is to study the effect of cell membrane polarization on the cell response to electroporation, and to compare these results obtained with whole cells to those reported in chapter 6 on BLM models for the apical and basolateral parts of cell membranes.

A cell membrane is a very heterogeneous structure; its composition cannot only differ between cells in the same tissue but also within a single cell. Two types of heterogeneities are discussed in chapter 6 where BLMs are prepared with a composition derived from that of real cell membranes. For that purpose, the differences in membrane composition of (i) the apical and the basolateral part of MDCK cells and (ii) healthy and cancerous liver cells are examined. This difference in membrane composition is shown in chapter 6 to affect the response to electroporation of the different leaflets. The altered response found for cancer cells or the apical of MDCK cells is likely to have an impact for many clinical applications, for instance during electrochemotherapy, if the same effect is observed in cells. Here, we aim at examining the possible effect of the heterogeneity within a single cell when working with whole cells and not membrane models. For this, MDCK cells whose membrane leaflets have been studied using BLM models are employed, and an experiment is designed to see whether the same difference in electroporation behavior as with BLMs is found in MDCK cells, i.e. that the apical part is more resistant to electroporation than the basolateral part. A miniaturized electroporation system, specifically designed for this application is employed. Firstly, the use of a miniaturized device brings enhanced control over the electroporation process. An overview of the advantages brought by miniaturized and microfluidic systems for (single) cell electroporation can be found in Appendix A, as well as the description of various microsystems developed for this purpose. In the present system discussed in this chapter, cells are grown as monolayers in $200-\mu \mathrm{L}$ wells made from PDMS either on hydrogel prepared on an integrated electrode (thin layer of iridium tin oxide (ITO)), or directly on the integrated electrode. They are possibly polarized as a function of the nature of the bottom surface, as illustrated in fig 8.1. The cells grown on ITO should not be polarized, as earlier reported for glass surfaces [1], and their membrane composition is expected to be "homogenized". On the contrary, on the hydrogel, the cell membrane should be organized in an apical part and a basolateral part: the cells should be polarized [2].

In both cases, pore formation starts on the side of the cell close to the anode as explained in chapter 2, i.e., in our settings, the side of the cell that is in contact with the solution and not the substrate. When cells are polarized, the apical part of the cells is in contact with the solution, and the first pores should therefore be created on that side of the cell. However, according to our preliminary studies employing BLM models, the apical part is more resistant to pore formation due to the higher concentration of glycolipids on that side of the cells, so polarized cells are consequently expected to electroporate later than non- 
polarized cells. For the latter cells grown in ITO, the membrane composition is homogenized, and the glycolipids are equally distributed in the whole cell membrane: the side of the cell in contact with the solution is expected to be less resistant to electroporation than the apical part of the polarized cells.

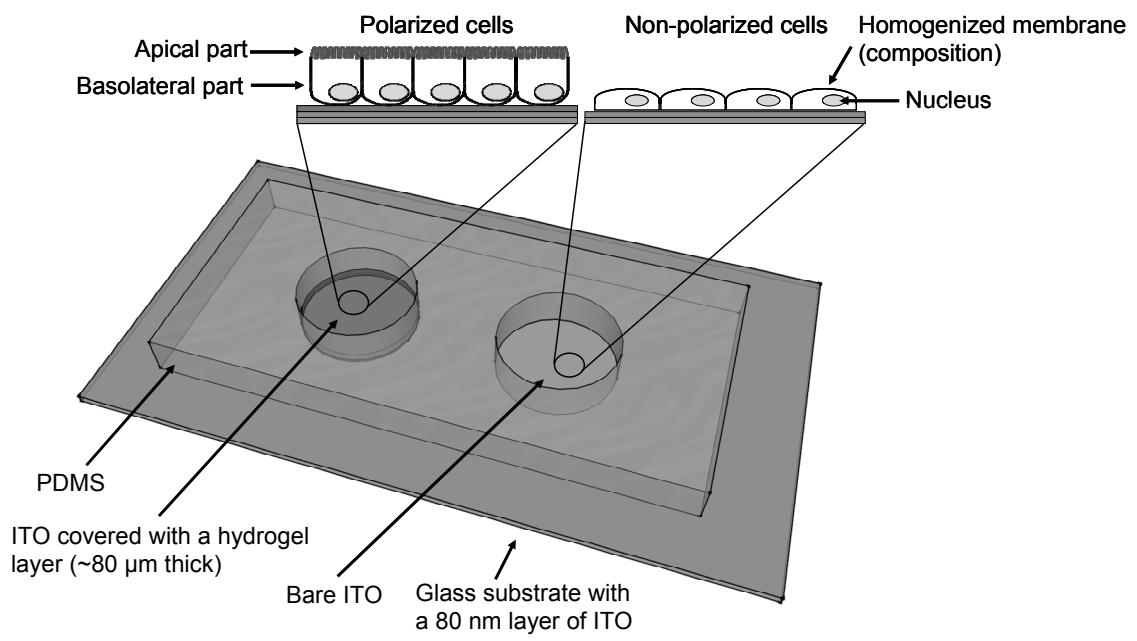

Figure 8.1. Schematic of the electroporation experiment.

Cells grown onto the layer of hydrogel in one of the PDMS wells (left) polarize (i.e. they have two distinct membrane parts, the apical (here indicated in red in the inset) and the basolateral), whereas cells grown onto the bare ITO display a homogenized membrane composition (right).

\section{Material and Methods}

\section{A. Chemicals}

Triton X-100, bovine serum albinum (BSA), matrigel (ECM Gel from Engelbreth-HolmSwarm murine sarcoma), inositol, calcium acetate, magnesiumacetate and l-histidine are purchased from Sigma Aldrich (Zwijndrecht, the Netherlands). MEM medium, fetal bovine serum (FBS), $2 \%$ penicillin, $1 \%$ L-glutamine and $0.16 \%$ fungizone are purchased from Invitrogen (Breda, the Netherlands). Texas Red-X phalloidin, FITC-conjugated goat antimouse IgG, goat serum, Hoechst, PI (propidium iodide) and calcein AM are purchased from Molecular Probes (Breda, the Netherlands). E-cadherin is purchased from BD Biosciences (Breda, the Netherlands). The conductive silver paint (electrolube) is purchased from Farnell (Utrecht, the Netherlands). The optical adhesive NOA-83H is purchased from Norland Products Inc. (Cranbury, New Jersey, USA). Deionized water $(18.2 \mathrm{M} \Omega \times \mathrm{cm})$ which is used for all solution preparation and cleaning procedure is obtained from a MilliQ Analysis system (Millipore, Billerica, MA). 


\section{B. Device description and fabrication}

\section{(i) Description of the microdevice}

The electroporation device developed for the electroporation of adherent cells consists of a series of wells (10-mm diameter) fabricated in PDMS and bonded to an ITO-coated glass substrate (see figure 8.2). Cells are grown as monolayers in the microwells. The layer of ITO forms the counter electrode in the design, whereas a steel plate electrode added from the top of the well is employed as the stimulation electrode. This latter electrode is positioned at a fixed distance $(1.8 \mathrm{~mm})$ to ensure the reproducibility of the electric field across the cells.

\section{(ii) Processing of the glass substrates}

A borosilicate glass wafer (Borofloat 33, 4 inch diameter, $500 \mu \mathrm{m}$ thickness) is first thoroughly cleaned in fuming $100 \% \mathrm{HNO}_{3}$ for $10 \mathrm{~min}$, rinsed in deionized water (DI water) and dried. In a next step, a layer of iridium tin oxide (ITO) is sputtered to a thickness of 80$100 \mathrm{~nm}$ (home-build sputtering apparatus) on the cleaned glass wafers. The resulting layer has a specific resistance of $\sim 100 \Omega / \mathrm{cm}^{2}$. As the glass substrate is used as small pieces of 2.5 $\mathrm{cm} \times 7.5 \mathrm{~cm}$, the whole wafer is subsequently diced using dedicated equipment (Disco DAD321 blade type TC 300, spindle rev. 25,000 rpm). During this dicing step, the wafers are protected by a Riston foil.

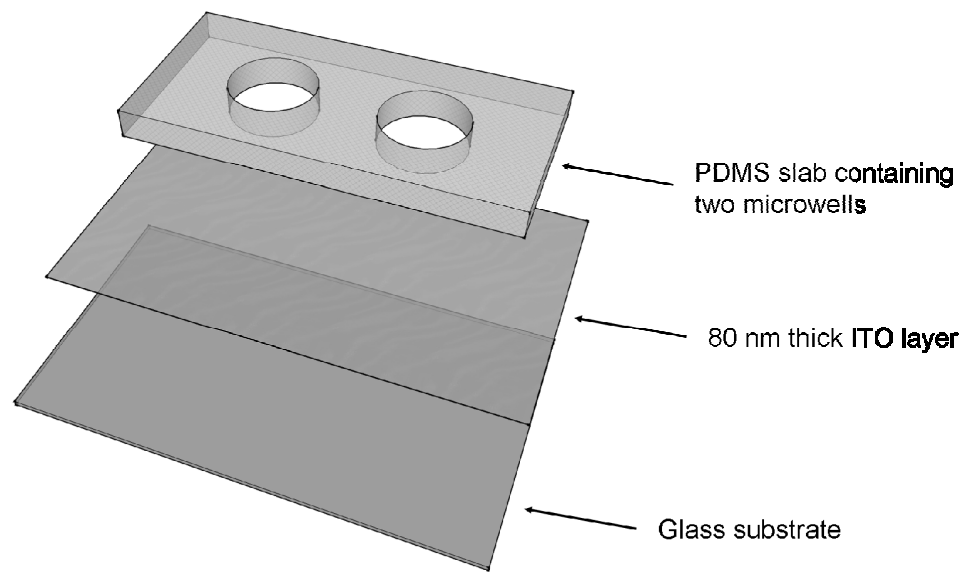

Figure 8.2. Miniaturized device for the electroporation of adherent cells.

Cells are grown in 10-mm diam. wells fabricated in PDMS. The PDMS layer is bonded on a glass substrate on which a layer of ITO has been sputtered to produce an integrated electrode for the electroporation.

\section{(iii) PDMS wells}

To form the PDMS layer, a bare silicon wafer is employed. This wafer is made hydrophobic with a FDTS coating (Perfluorodecyltrichlorosilane, ABCR GmbH \& Co. KG, Karlsruhe) before pouring the PDMS. This coating prevents the PDMS from sticking to the silicon substrate and facilitates removal of the PDMS. 
A two-component kit (Sylgard 184, Dow Corning, Midland, Michigan, USA) is used for PDMS fabrication; it includes a pre-polymer of PDMS as well as curing agent. They are mixed in a 10:1 weight ratio, the resulting mixture thoroughly degassed in vacuum, subsequently poured into the silicon master, anew degassed in vacuum and cured for $2 \mathrm{~h}$ at $80^{\circ} \mathrm{C}$. After cooling, the PDMS device (having a thickness of $\sim 40 \mathrm{~mm}$ ) is peeled-off from the silicon wafer.

To form the wells into the PDMS slab, a home-made punching tool is employed with an outer diameter of $1 \mathrm{~cm}$. As the devices are fabricated at the chip level, the PDMS substrate containing the wells is cut in individual pieces using a sharp knife.

\section{(iv) Packaging}

The final devices are produced by bonding the PDMS layer onto the ITO-coated glass substrates. Since bonding of PDMS on ITO-coated surfaces is not successful using standard plasma treatment, a derivative of the technique recently developed in our lab is employed. This bonding technique is similar to the method described in chapter 7 and relies on the use of an intermediate layer of glue [3] which is transferred from a dummy substrate onto one of the surfaces to be bonded together. This approach is flexible; it can easily be adapted as a function of both the nature of the materials to be assembled and the size of the (micro)structures. Here, NOA- $83 \mathrm{H}$ is employed as gluing material; this resin can be cured thermally (between $\left.60-125^{\circ} \mathrm{C}\right)$ or by exposure to UV light $(\lambda=320-380 \mathrm{~nm}$, with a sensitivity peak around $\lambda=365 \mathrm{~nm}$ ). Prior to bonding, the ITO and PDMS substrates are cleaned in isopropanol (IPA) for $10 \mathrm{~min}$ in an ultrasonic bath and carefully dried, first with compressed air and subsequently for $30 \mathrm{~min}$ at $60^{\circ} \mathrm{C}$. The resin $\mathrm{NOA}-83 \mathrm{H}$ is spread on a cleaned glass microscope slide. The PDMS parts are stamped onto this layer of glue and subsequently placed on the ITO substrates. Since no additional structures are present, careful alignment of the two parts is not required. Finally, the assembled device is exposed for at least $2 \mathrm{~h}$ to UV light (366 nm; $1 \mathrm{~mW}$; NU-6KL, Konrad Benda Laborgeräte, Wiesloch, Germany) to completely cure the NOA- $83 \mathrm{H}$ resin and bond thereby the two layers together. Such a long curing time is necessary to ensure proper curing of the optical glue as the light has to go through the ITO, resulting in a loss of power.

\section{(v) Addition of a hydrogel layer}

For half of the experiments, hydrogel is added in the microwells to induce cell polarization. Before addition of the hydrogel, the devices are incubated in a $5 \mathrm{mg} / \mathrm{mL}$ BSA solution overnight to make the walls permanently hydrophilic; this prevents the hydrogel from being attracted to the PDMS. The next day, the wells are dried under a nitrogen flow and a small amount $(25 \mu \mathrm{L})$ of hydrogel (matrigel, ECM Gel from Engelbreth-Holm-Swarm murine sarcoma) is added to the wells $\sim 5 \mathrm{~min}$ before seeding of the cells. The hydrogel jellifies within $5 \mathrm{~min}$ when placed at $20^{\circ} \mathrm{C}$, so that care has to be taken to manipulate the gel at a low temperature. For that purpose, the electroporation devices are placed on a block of ice before the gel is added and cold pipette tips are employed to dispense the gel into the 
wells.

\section{Cell experimentation}

\section{(i) Cell culture}

MDCK cells are obtained from the Max-Planck-Institute of Molecular Cell Biology and Genetics (Dresden, Germany) and cultured in $5 \% \mathrm{CO}_{2}$ at $37^{\circ} \mathrm{C}$ in Eagle's Minimal essential medium (MEM) (Invitrogen, Breda, the Netherlands) supplemented with 10\% fetal bovine serum (FBS) (Invitrogen), 2\% penicillin, 1\% L-glutamine, $0.16 \%$ fungizone. Cells are harvested by incubation with $1.25 \%$ (w/v) trypsin (Invitrogen) after which complete growth medium is added to inactivate the trypsin. Following this, the cells are centrifuged and resuspended in complete culture medium at a concentration of either $1.8 \times 10^{5}$ or $3.6 \times 10^{5}$ cells $/ \mathrm{mL}$. $200 \mu \mathrm{L}$ of this cell suspension is added into the wells (both on ITO and on hydrogel). Cells are cultured in the wells for $1-4$ days (in $5 \% \mathrm{CO}_{2}$ at $37^{\circ} \mathrm{C}$ ), depending on their initial concentration until a nice and homogeneous monolayer is obtained. The media in the wells is refreshed every 2 - 3 days.

\section{(ii) Cell staining for electroporation experiments and immunofluorescence}

Prior to the electroporation experiments, cells are stained with calcein AM (Molecular Probes, Breda, The Netherlands). MDCK cells are incubated with $0.4 \mu \mathrm{L}$ calcein AM solution $(1 \mathrm{mM})$ for $1 \mathrm{~h}$ (at $37^{\circ} \mathrm{C}, 5 \% \mathrm{CO}_{2}$ ) and subsequently washed twice with PBS (phosphatebuffered saline) after which complete culture medium is added into the wells. After the electroporation experiment, the wells are again filled with complete culture medium to allow the cell membranes to reseal. Cell viability is checked after $30 \mathrm{~min}$ with the membrane integrity marker PI (final concentration $0.01 \mu \mathrm{g} / \mathrm{mL}$ ).

To determine if the cells are polarized, specific stains are employed: phalloidin coupled to Texas Red (Molecular Probes, Breda, the Netherlands) to stain actin found in the apical membrane part and anti-E-cadherin (followed by a FITC coupled to a secondary antibody) that should accumulate in the basolateral part of the membrane. The separation between the two dyes in the different membrane regions should be an indication for membrane polarization [2].

To stain the cells with the specific antibodies, MDCK cells are fixed with $3 \%$ paraformaldehyde (15 min incubation), washed three times with PBS, permeabilized with 0.1\% Triton X-100 for $2 \mathrm{~min}$, and blocked with 20\% goat serum in PBS for $1 \mathrm{~h}$. The fixed and permeabilized cells are incubated with E-cadherin mAb $(5 \mu \mathrm{g} / \mathrm{mL})$ and Texas Red-X phalloidin ( 5 units $/ \mathrm{mL}$ ) in PBS containing 1\% BSA, for $1 \mathrm{~h}$, followed by staining with FITCconjugated goat anti-mouse IgG secondary antibody $(5 \mu \mathrm{g} / \mathrm{mL}$, Molecular Probes, Eugene, OR) in PBS containing 10\% goat serum for $1 \mathrm{~h}$. In a last step, the nuclei of the cells are stained with Hoechst by incubating the cell in complete medium with $0.4 \mu \mathrm{L}$ of Hoechst solution $(1 \mathrm{mg} / \mathrm{mL})$ for $30 \mathrm{~min}$. After each incubation step, cells are washed with PBS three times, and each washing step lasts $1 \mathrm{~min}$. 


\section{(iii) Cell imaging using confocal microscopy}

PDMS wells are bonded to a thin glass substrate to examine cell polarization using confocal microscopy (Zeiss LSM 510-meta system) and a Plan-Apochromat 63x/1.4 Oil DIC objective. Excitation wavelengths and filters are as follows: FITC, $488 \mathrm{~nm}$ excitation, emission BP 500-500 nm filter; Texas-Red, $543 \mathrm{~nm}$ excitation, emission LP $560 \mathrm{~nm}$ filter.

\section{Electroporation experiments}

For the electroporation experiments, the device with the microwells is placed on the stage of an inverted microscope (IX51, Olympus, Center Valley, PA) equipped with appropriate filters to optically detect electroporation (see figure 8.3). A thin steel plate is cut with a laser into a round shape with a stem. The round section ( $8.5 \mathrm{~mm}$ diameter), fits snugly into the PDMS wells. The round part is bent to an angle of $90^{\circ}$ with respect to the stem for fastening the electrode into a homemade $\mu \mathrm{m}$-manipulator that allows for moving the steel plate electrode up and down. This system is mounted on top of a base plate that is positioned around the chip so that the electrode aligns with the well (see figure 8.3). The electrode is lowered into the buffer solution at a fixed distance above the cells, so that on one hand the applied potential can be as low as possible, and on the other hand the cells are not affected by the shear stress generated by the electrode insertion.

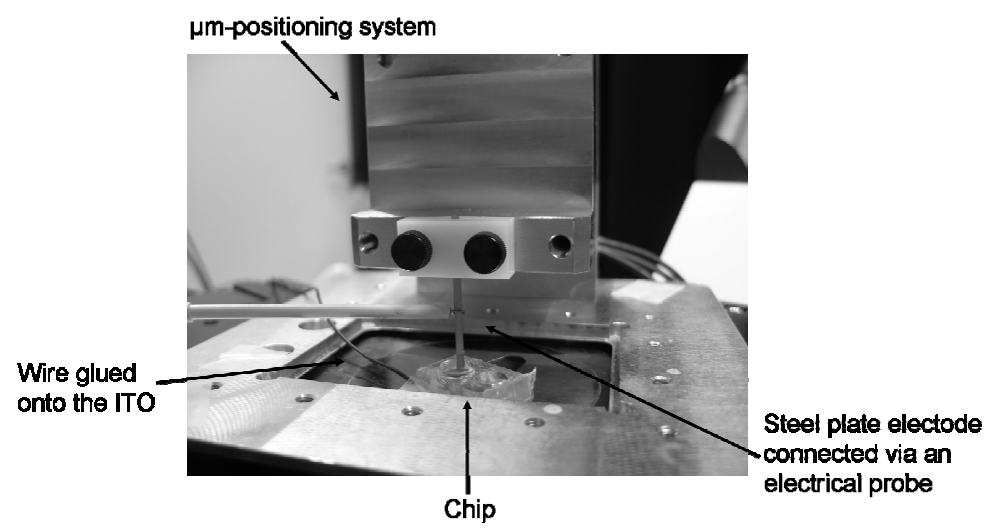

Figure 8.3. Picture of the electroporation set-up

A homemade $\mu$ m-positioning system is placed on top of an inverted microscope. The electrode is lowered into the buffer solution in one of the wells and the electrodes are connected to the pulse generator with the help of electrical clamps.

ITO is electrically connected with a thin wire is glued onto the device surface with conductive silver paint (electrolube). After drying of the glue, the well is filled with a low conductive electroporation buffer (iso-osmolar buffer with a conductivity of $9 \mathrm{mS} / \mathrm{m}$ : 280 $\mathrm{mM}$ inositol, $0.1 \mathrm{mM}$ calcium acetate, $0.5 \mathrm{mM}$ magnesium acetate and $1 \mathrm{mM}$ l-histidine). Once the electrode is in position, 8 pulses of $20 \mathrm{~ms}$ (at $25 \mathrm{~Hz}$ ) and a $5-9 \mathrm{~V}$ amplitude are 
applied every 5 min using a pulse generator (model 500, Berkeley Nucleonics Corporation, San Rafael, CA) and measured with an oscilloscope (54621A, Agilent, Santa Clara, CA). The decrease is fluorescence is checked prior to the application of every new pulse train. When an extensive release of calcein is detected, cells are washed prior to imaging to reduce the background signal and enable quantitative fluorescent measurements.

\section{E. Data analysis}

The changes in fluorescent intensity for the electroporation experiments are analyzed using the freeware ImageJ (National Institute of Health). Ten individual cells are randomly selected and their fluorescent intensity measured for the different pictures taken after each pulse train. Resulting data are processed in Excel (Microsoft). The confocal microscopy pictures are analyzed using Zen software from the confocal microscope, and processed with Corel PHOTO-PAINT.

\section{Results}

In the coming section, firstly the characteristics of the electroporation system are described. Following, the responses to electroporation for cells grown on hydrogel and bare ITO are compared. Lastly, the results for the membrane polarization on the different surfaces are discussed.

\section{A. Characteristics of the electroporation and measurement set-up}

\section{(i) Miniaturized electroporation devices}

In this part of the chapter, the novel miniaturized electroporation device specifically designed to study the effect of cell polarization on their response to electroporation is discussed in more detail. The design and the fabrication process of this miniaturized electroporation tool itself is straightforward. It consists of a PDMS layer including microwells and bonded to a glass substrate coated with a thin layer of ITO. As the bonding step requires no precise alignment between the ITO layers and the PDMS the systems are very easy to produce. In these devices, MDCK cells are grown as a monolayer on a thin hydrogel layer prepared in the microwell or directly on bare ITO itself. As a second electrode, a disk-shaped structure made from stainless steel is employed; it is brought in the device from the top of the well and lowered into the solution placed in the well.

Two aspects are particularly critical for the system production and utilization: the thickness of the hydrogel layers and the electrode configuration (design, polarity of the signal, and distance between electrodes), which are hereafter discussed separately.

\section{(ii) Hydrogel formation}

The hydrogel layer must be as thin as possible to facilitate optical imaging of the cells in the course of the electroporation experiments. In a first attempt, $100 \mu \mathrm{L}$ of hydrogel was used to prepare the hydrogel layer in every well. However, this gives a too thick layer (several hundreds of $\mu \mathrm{m}$ in thickness), and imaging of cells is hindered: pictures are blurred 
and out-of-focus. Subsequently, the amount of hydrogel is progressively decreased to the smallest amount possible; $25 \mu \mathrm{L}$ of hydrogel per well is found optimum for both the formation of a homogeneous layer of gel in the wells and satisfactory imaging conditions.

To determine the thickness and smoothness of the hydrogel layers, the liquid matrigel is supplemented with a fluorescent dye (fluorescein disodium salt (Sigma Aldrich (Zwijndrecht, the Netherlands))) prior to its addition to the wells, resulting in the formation of a fluorescent hydrogel layer. These layers are characterized using confocal microscopy to determine their thickness. Figure 8.4 illustrates the typical profile obtained for a part of one layer of hydrogel. The resulting layers are smooth and flat and their thickness is $70 \pm 14 \mu \mathrm{m}$ for a volume of $25 \mu \mathrm{L}$ hydrogel added into the wells.
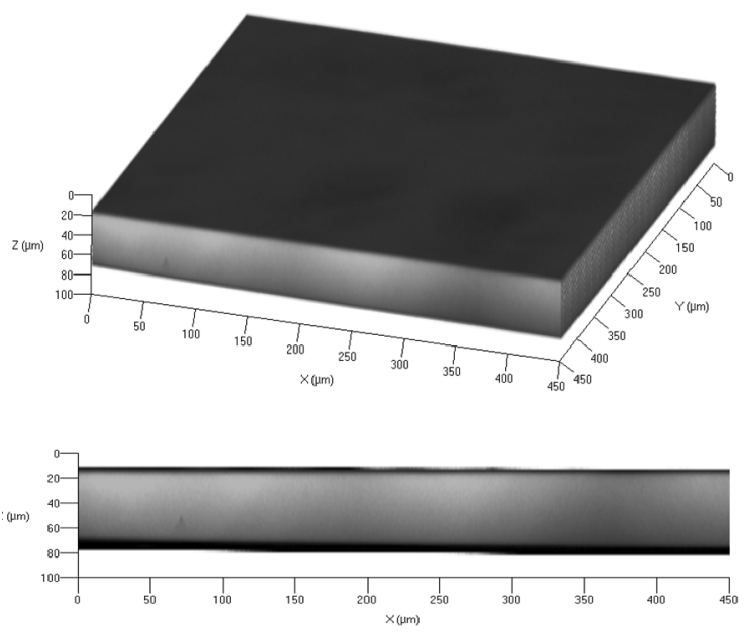

Figure 8.4. Fluorescent hydrogel layer

$3 D$-view (top) and side view (bottom) of a $450 \mu \mathrm{m} \times 450 \mu \mathrm{m}$ area of a fluorescent hydrogel layer (25 $\mu \mathrm{L}$ hydrogel supplemented with fluorescein disodium salt) in a PDMS well. The thickness of this layer is $70 \pm 14 \mu \mathrm{m}$.

\section{(iii) Electrode configuration}

The second crucial feature in this set-up is the configuration of the electrodes, and this includes three different aspects: the distribution of the electric field across the cell monolayer, the polarity of the signal, and the distance between the electrodes. For electroporation experiments, two electrodes are employed. As already mentioned, one is integrated in the microdevice and consists of a continuous ITO layer on the glass bottom substrate of the device, while the second electrode is external and inserted in the well from its top (see figure 8.5).

Firstly, as a result of this geometry, the electrodes can be considered as two infinite plans with respect to the cells. Consequently, when a voltage is applied, the resulting electric field between them is homogeneous and orthogonal to the two planes, as illustrated in 
figure 8.5. This means that the electric field distribution in the device can be modeled easily as the field lines run from the top to the bottom electrode in straight lines. Only on the edge, the E-field distribution is disturbed, but measurements are performed in the middle of the well, so that this edge effect can be neglected.

On other aspects, the polarity of the electrodes is chosen to "test" the effect of the cell polarization on the outcome of the electroporation process. As already mentioned in the introduction, the polarity of the applied signal dictates the side of the cell where the process of pore formation is initiated. As detailed in chapter 2 of this thesis, pores are first created on the side of the cell where the trans-membrane potential reaches first its critical value, i.e. the side of the cell facing the anode. This effect is a result of the negative internal potential of the cell, which determines on which side of the cell the largest transmembrane potential arises depending on the applied potential. The goal of these experiments is to assess the response of the cells to the applied electric field when the cells are polarized and when cells are not polarized. This enables to determine the sensitivity of the apical membrane to the electric field. The anode is placed in solution (top electrode), while the cathode corresponds to the ITO-based integrated electrode. When cells are polarized (hydrogel surface), with this configuration, we expect that pore formation should start in the more resistant apical side of the cell while when not polarized (bare ITO surface), pore formation should initiate in a homogenized and less resistant membrane.

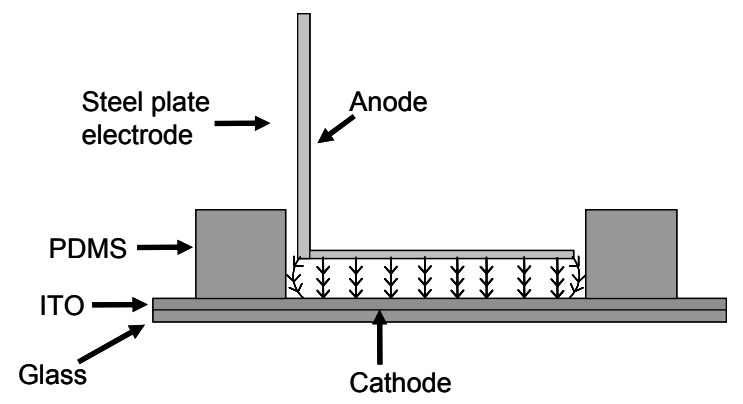

Figure 8..5 Electric field distribution

Electric field distribution between the planar steel electrode and the ITO bottom electrode. The field lines are homogeneous and straight underneath the top electrodes, where the experiments are performed.

Last, the electrode should always be placed at the same distance from the ITO surface to generate reproducible electric fields in all experiments. When the distance between the two electrodes is fixed, all cells in the monolayer are exposed to the same electric field since their position is fixed as well (which is a large advantage over the electroporation of suspension cells). This increases both the control over the electroporation process and the reproducibility of the experimental outcome. The disk-shaped electrode (see figure 8.5 ) is 
lowered into the buffer inside the well using a $\mu$ m-positioning system until the arbitrary chosen position (1.8 mm above the ITO surface) is reached. The position of the electrode in the $z$-direction is finely tuned with the help of a $\mu \mathrm{m}$-precision screw, and the position carefully noted so that the same value is employed for all experiments. The distance between the two electrodes is determined to be $1.8 \pm 0.05 \mathrm{~mm}$, which results in an electric field of $2.8 \times 10^{-2}-5 \times 10^{-2} \mathrm{kV} / \mathrm{cm}$, depending on the applied potential.

However, in this configuration, the presence of the hydrogel is neglected as well as its influence on the electric field distribution, since the conductivity of the hydrogel is much higher than that of the buffer employed for the electroporation experiments. Taking into account that the height of the hydrogel is only $70 \mu \mathrm{m}$ while the distance between the electrodes is $1.8 \mathrm{~mm}$, the change in resistance in the device is calculated to be of only $4 \%$. This small change is ignored here for the positioning of the second electrode.

\section{B. Electroporation of monolayers of MDCK cells}

Here, we study the effect of the membrane polarization of MDCK cells on their response to electroporation. The hypothesis behind these experiments is that the apical membrane part of the polarized MDCK cells is more resistant to pore formation than the membrane of non-polarized cells whereas the basolateral part is less resistant due to the altered amounts of glycolipids present there.

MDCK cells are cultured on different surfaces (hydrogel or ITO) to induce or not their polarization. Thereby, the distinction between polarized and non-polarized cells is possible. ITO also serves as one electrode, and the other electrode, a stainless steel disk, is introduced in the solution. Electroporation is monitored optically using a standard protocol [4-24]. Cells are previously stained with the membrane integrity marker calcein before they are exposed to the electric pulses. When the membrane is porated, the green fluorescent dye is released and the fluorescent intensity of the cells decreases progressively. The decrease in fluorescence is measured for at least 10 individual cells in the field of view of the microscope, once the cells have been subjected to the electroporation treatment. On other aspects, 30 minutes after the electroporation assay, another membrane integrity marker, PI, is added to the solution to check whether cells are still viable. Cells that become positive to PI are dead, and have been irreversibly electroporated.

Firstly, the parameters of the electroporation treatment are determined. Pulse trains (8 pulses of $20 \mathrm{~ms}$ at $25 \mathrm{kHz}$ ) with an increasing amplitude starting from $2 \mathrm{~V}$ (or a field strength of $1.12 \times 10^{-2} \mathrm{kV} / \mathrm{cm}$ ) and with a step of $1 \mathrm{~V}$ are applied every 5 minutes, until electroporation is detected. Cells grown onto bare ITO show calcein release for a pulse amplitude of $5.2 \mathrm{~V} \pm 1 \mathrm{~V}$ (10 experiments) against $6.8 \mathrm{~V} \pm 1.7 \mathrm{~V}$ (7 experiments) for cells on the hydrogel. The nature of the substrate on which cells are seeded has subsequently an influence in their sensitivity to electroporation, and cells grown on the hydrogel layer require higher pulse amplitudes to become electroporated. It should be noted that the variation in the value of the voltage amplitude is relatively large compared to the actual 
values (up to $25 \%$ from the measured value), and this most probably reflects the quality of the cell monolayer, as discussed later in more detail in this chapter: non confluent monolayers require a higher signal amplitude as the holes in the monolayer act as leakage pathways for the current, and depending on the precise location of the cell in the monolayer (isolated cell or cell in a dense area), the electrical treatment is different.

To test this difference even further, in a next series of experiments, multiple pulse trains with the same amplitude are applied every $5 \mathrm{~min}$; this time interval is chosen to allow pores to close again before cells are exposed to another electrical treatment. The fluorescence variation upon electroporation is monitored to confirm that there is a steady decrease in the fluorescent intensity when the electroporation treatment is repeated, and that this decrease is not due to an experimental artifact. Cells are exposed to 6 pulse trains (8 pulses of $20 \mathrm{~ms}$ ) with a time interval of $5 \mathrm{~min}$, and the pulse amplitude is varied for both surfaces between 5 and $9 \mathrm{~V}$ (or a field strength of $2.8 \times 10^{-2}-5 \times 10^{-2} \mathrm{kV} / \mathrm{cm}$ ). It should be noted that care is taken during all experiments not to expose the cells continuously to the UV light as calcein is highly photosensitive: the $\mathrm{Hg}$ lamp is turned on only to take pictures after application of every pulse train. Besides, photobleaching is quantified using stained MDCK cells grown on both surfaces. There, cells are not exposed to any electrical treatment but pictures are taken every $5 \mathrm{~min}$ over a period of $30 \mathrm{~min}$ as during the electroporation assays. Figure 8.6 shows the results of this preliminary assay for 10 different cells on both types of surfaces. The loss of fluorescence due to photobleaching is quantified as $20 \%$ for both types of substrates, for an assay of $30 \mathrm{~min}$ (7 pictures, approximately $5 \mathrm{sec}$ UV exposure per picture). As expected, the amount of dead cells in the photobleaching assay is very low ( 1 cell per 260-280 cells) in this experiment.

When electric pulses are applied two outcomes are possible with respect to the fluorescent intensity of the cells: (i) either no significant difference is measured compared to the photobleaching results, and in that case cells are assumed not to be electroporated (or pores are too small for calcein to be released), (ii) or a decrease greater than $20 \%$ is measured, and in the latter case, pores are formed in the cell membranes. As previously observed, electroporation is detected for the two substrates at different electric field strengths. Cells grown on ITO display a significant loss in their green fluorescence (between 25 and $80 \%$ ) upon application of $5 \mathrm{~V}$ pulses while for cells on a hydrogel layer the pattern in fluorescence variation (figure 8.7A) is very similar to that obtained for mere photobleaching (figure 8.6A). Cells grown on ITO can be classified in three groups depending on the calcein release upon electroporarion. One group displays a total fluorescence decrease of $25-30 \%$ which is slightly more than obtained in the photobleaching assay, one cell (represented by 
A

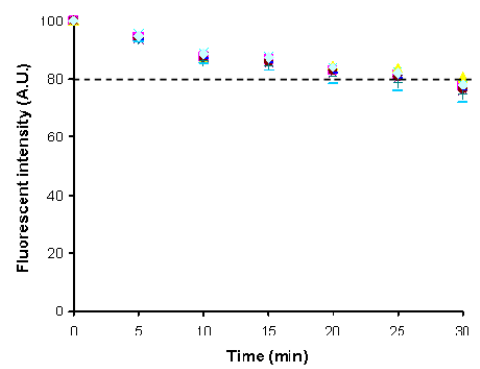

\section{Hydrogel}

$\mathrm{B}$
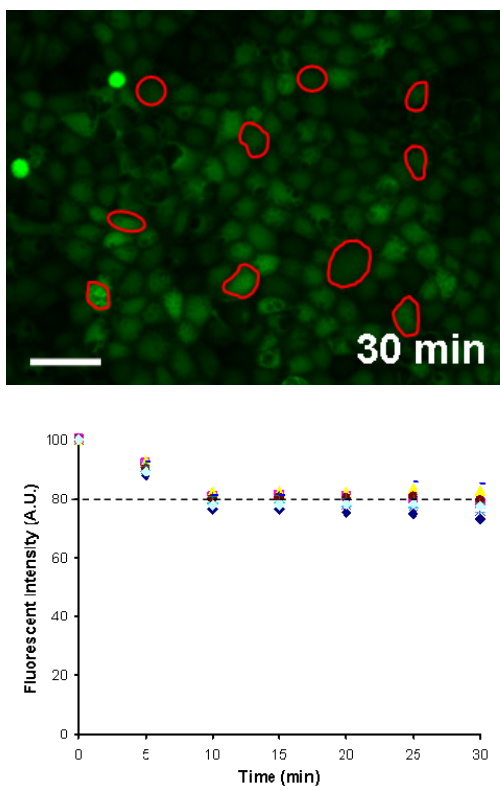

ITO

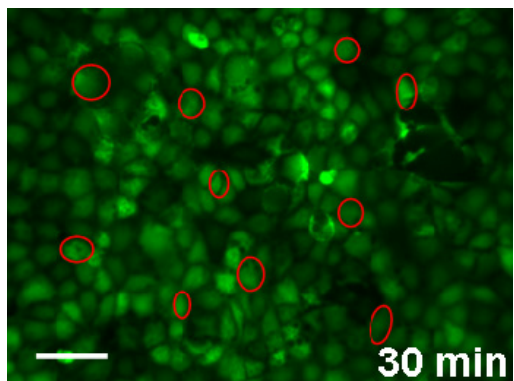

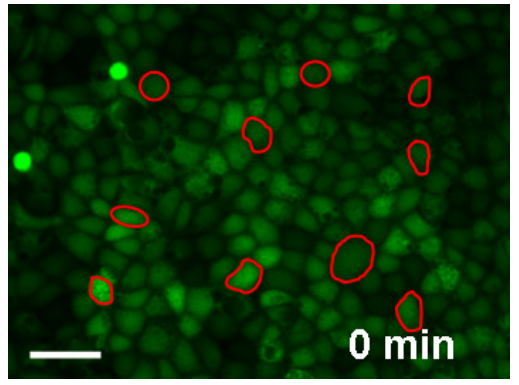
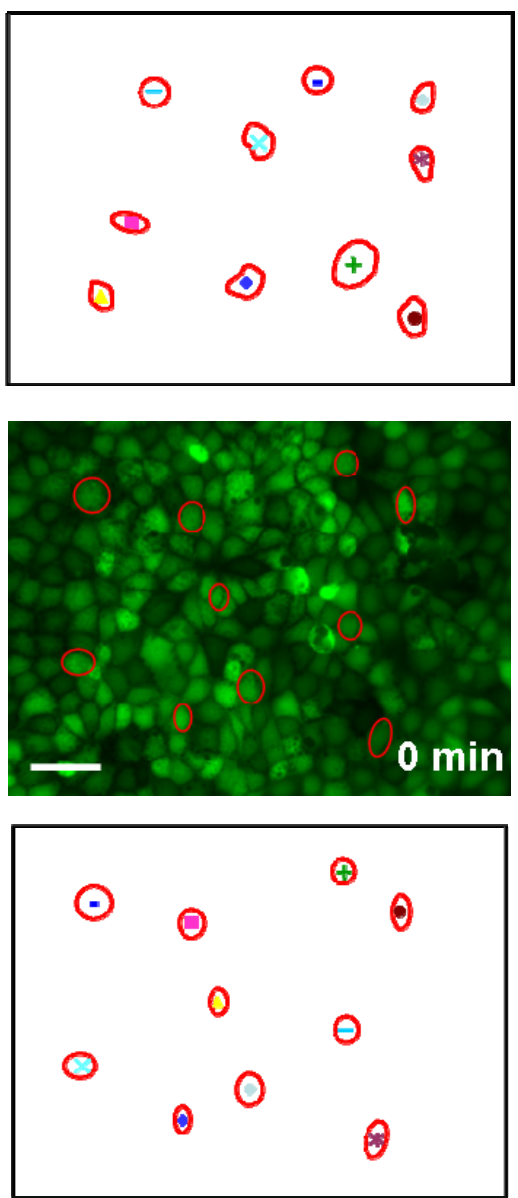

Figure 8.6. Photobleaching

Quantification of the photobleaching of calcein in MDCK cells grown on hydrogel (A) and bare ITO (B). Pictures of the MDCK cell monolayers at 0 and $30 \mathrm{~min}$ (top right, bottom left), and graphs (top, left) showing the decrease in the fluorescence intensity for 10 individual cells when pictures are taken every 5 min (bottom). These 10 cells are indicated with red circles and the symbols representing them in the graph (bottom, right). The striped line in the graph indicates the average loss of fluorescence (20\%) caused by photobleaching. Scale bar of $50 \mu \mathrm{m}$. 
the dark blue diamond) a decrease of $\sim 50 \%$, and a second group of cells a much larger decrease of $\sim 70 \%$. This significant discrepancy in the cell response to electroporation most probably reflects the heterogeneity of the cell population, the difference in cell sensitivity to the applied electrical treatment and the quality of the monolayer. Lastly, the amount of dead cells in the monolayer at the end of the experiment (30 min after the application of the last pulse train) was checked. As expected from the quite significant loss of fluorescence $(>80 \%)$ for some cells grown on ITO, some cells die during the electroporation treatment, and the amount of dead cells was measured to be of 24 dead cells per 260-280 cells. However, this amount of dead cells was also unexpectedly high for the hydrogel samples (10 dead cells per 260-280 cells in the whole imaging area, or $8.4 \times 10^{4} \mu \mathrm{m}^{2}$ against typically 1 cell for other control experiments) even though no electroporation was detected. This could indicate that the cells were already dying at the beginning of the experiment and that there may be additional electrical and/or mechanical stress imposed on the cells.

When the pulse amplitude is increased to $7 \mathrm{~V}$, some cells grown onto the hydrogel layer are porated: 7 out of the 10 cells considered give a decrease of more than $20 \%$, and up to $40 \%$, and a few remain unaffected by the electric field ( 3 cells), as shown in the graph and the pictures presented in figure $8.8 \mathrm{~A}$. As before, this difference in electroporation response reflects the fact that the cell population is not homogeneous, and that the cell monolayer is not confluent, presenting regions of higher or lower cell density. All cells on the ITO substrate are again electroporated, and they exhibit a large spread in the actual fluorescence decrease (between 20 and 90\%). Still, this decrease is on average much larger than for the $5 \mathrm{~V}$ treatment, and cells can be divided into two groups: one with a moderate $(\sim 25 \%)$ and one with a strong decrease $(\sim 80 \%)$. This difference in loss of fluorescence again reflects the heterogeneity of the cell population, and the difference in cell sensitivity and the monolayer quality. It should be noted that cells on ITO had to be washed in this series of experiments before taking pictures, after every pulse train application, to remove the released calcein and the resulting fluorescence background. As a consequence, part of the monolayer was damaged, as seen on the second picture of figure $8.8 \mathrm{~B}$. Furthermore, the graph in figure 8.8 B contains aberrant data: two cells (blue diamond and pink square) give a sudden increase in their fluorescence level between 10 and $15 \mathrm{~min}$, and this comes back to normal for the next measurement, and some cells (blue diamond, pink square, brown circle and purple asterisk) also exhibit the same phenomenon at the end of the experiment (at $30 \mathrm{~min}$ ). This sudden difference is most likely also caused by problems with the washing steps. The amount of dead cells at the end of the experiment is found to be higher than for a $5 \mathrm{~V}$ treatment: 36 and 15 dead cells (per 260-280 cells), for the ITO and hydrogel samples, respectively. 
A
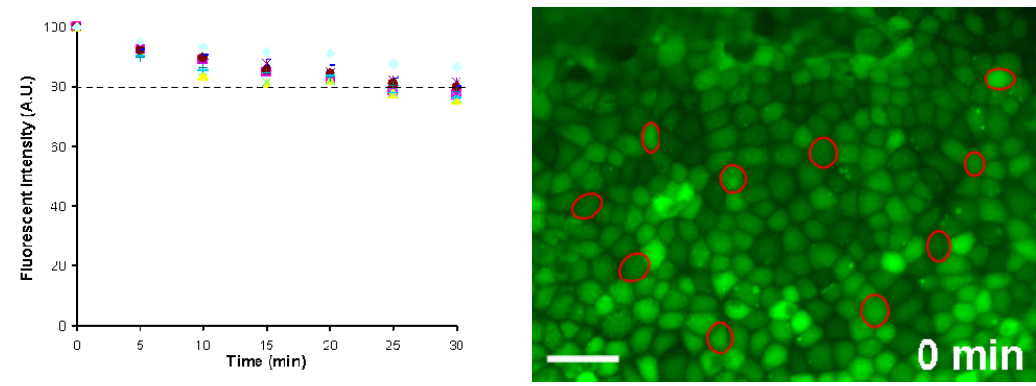

\section{Hydrogel}

B
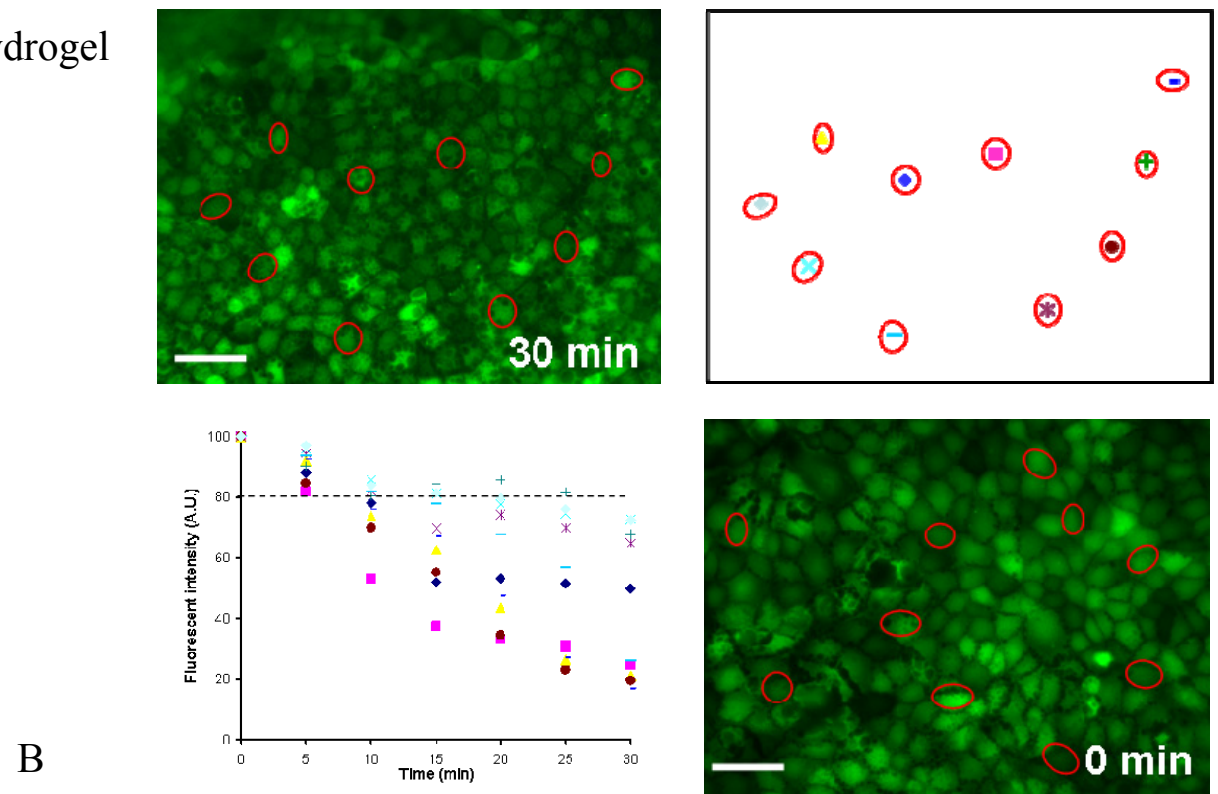

ITO
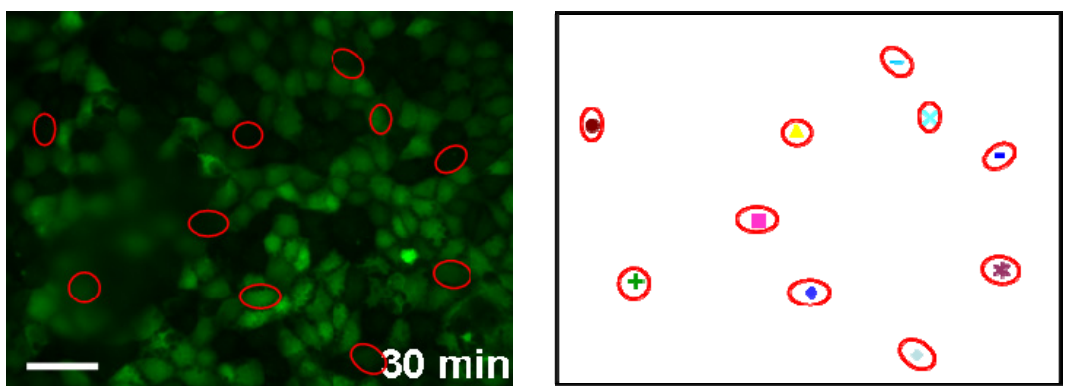

Figure 8.7. MDCK cell electroporation using a pulse amplitude of $5 \mathrm{~V}$

Quantification of the calcein release caused by electroporation in MDCK cells (8 pulses, $20 \mathrm{~ms}, 5 \mathrm{~V}$, treatment repeated every $5 \mathrm{~min}$ ) on hydrogel (A) and bare ITO (B). Pictures of the MDCK cell monolayers at 0 and $30 \mathrm{~min}$ (top right, bottom left), and graphs (top, left) showing the decrease in the fluorescence intensity for 10 individual cells when pictures are taken every $5 \mathrm{~min}$ (bottom). These 10 cells are indicated with red circles and the symbols representing them in the graph (bottom, right). The striped line in the graph indicates the average loss of fluorescence $(20 \%)$ caused by photobleaching. Scale bar of $50 \mu \mathrm{m}$. 
A
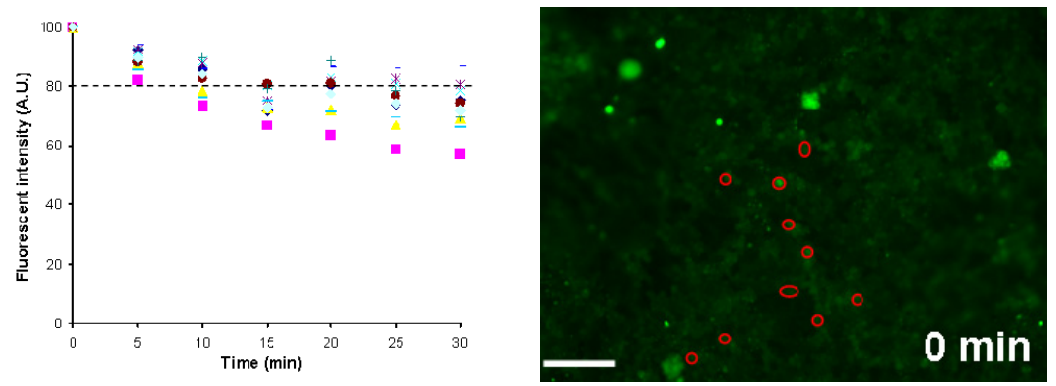

\section{Hydrogel}
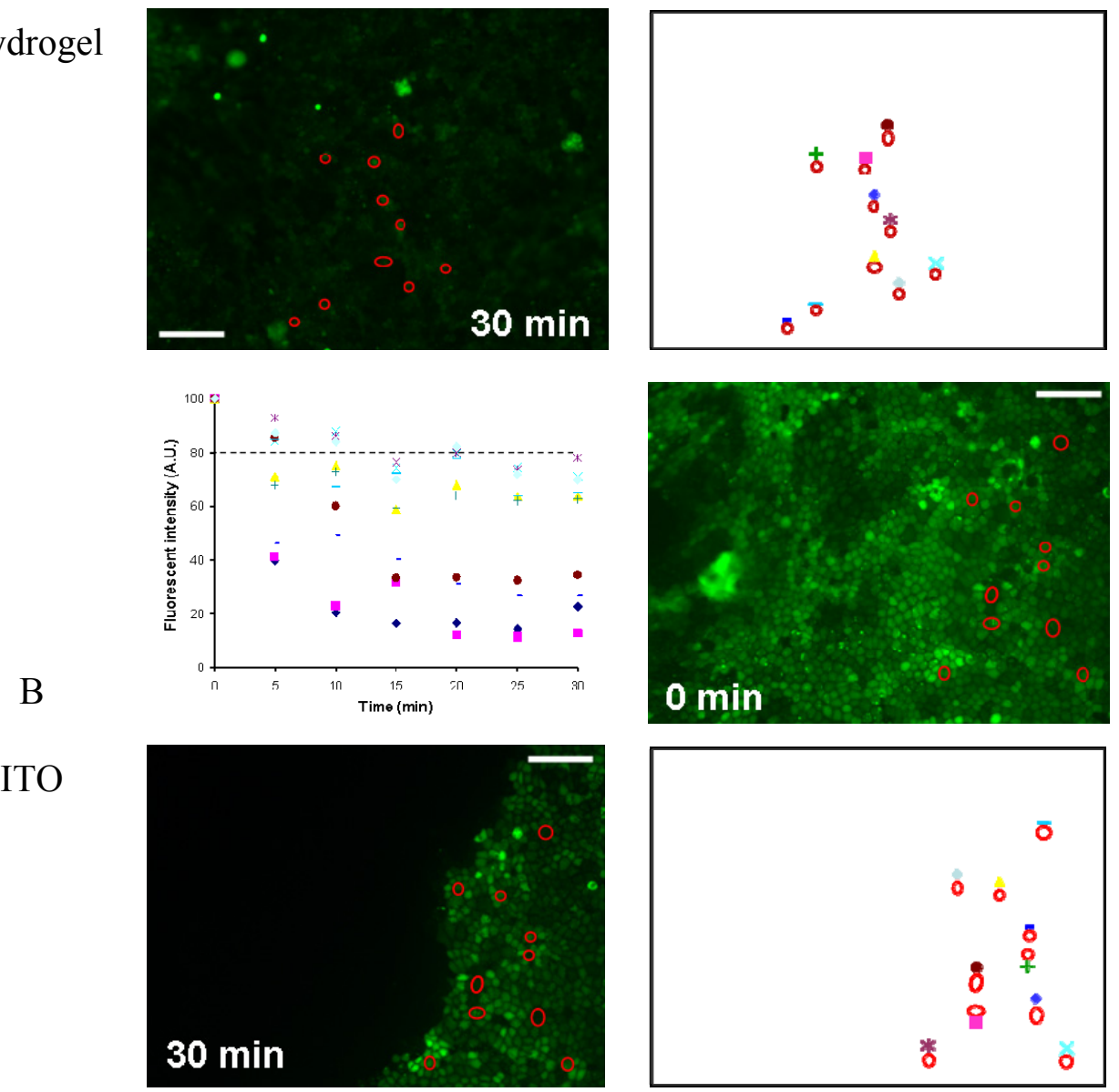

Figure 8.8. MDCK cell electroporation using a pulse amplitude of $7 \mathrm{~V}$

Quantification of the calcein release caused by electroporation in MDCK cells (8 pulses, $20 \mathrm{~ms}, 7 \mathrm{~V}$, treatment repeated every $5 \mathrm{~min}$ ) on hydrogel (A) and bare ITO (B). Pictures of the MDCK cell monolayers at 0 and $30 \mathrm{~min}$ (top right, bottom left), and graphs (top, left) showing the decrease in the fluorescence intensity for 10 individual cells. These 10 cells are indicated with red circles and the symbols representing them in the graph (bottom, right). The striped line in the graph indicates the average loss of fluorescence (20\%) caused by photobleaching. Scale bar of $50 \mu \mathrm{m}$. 

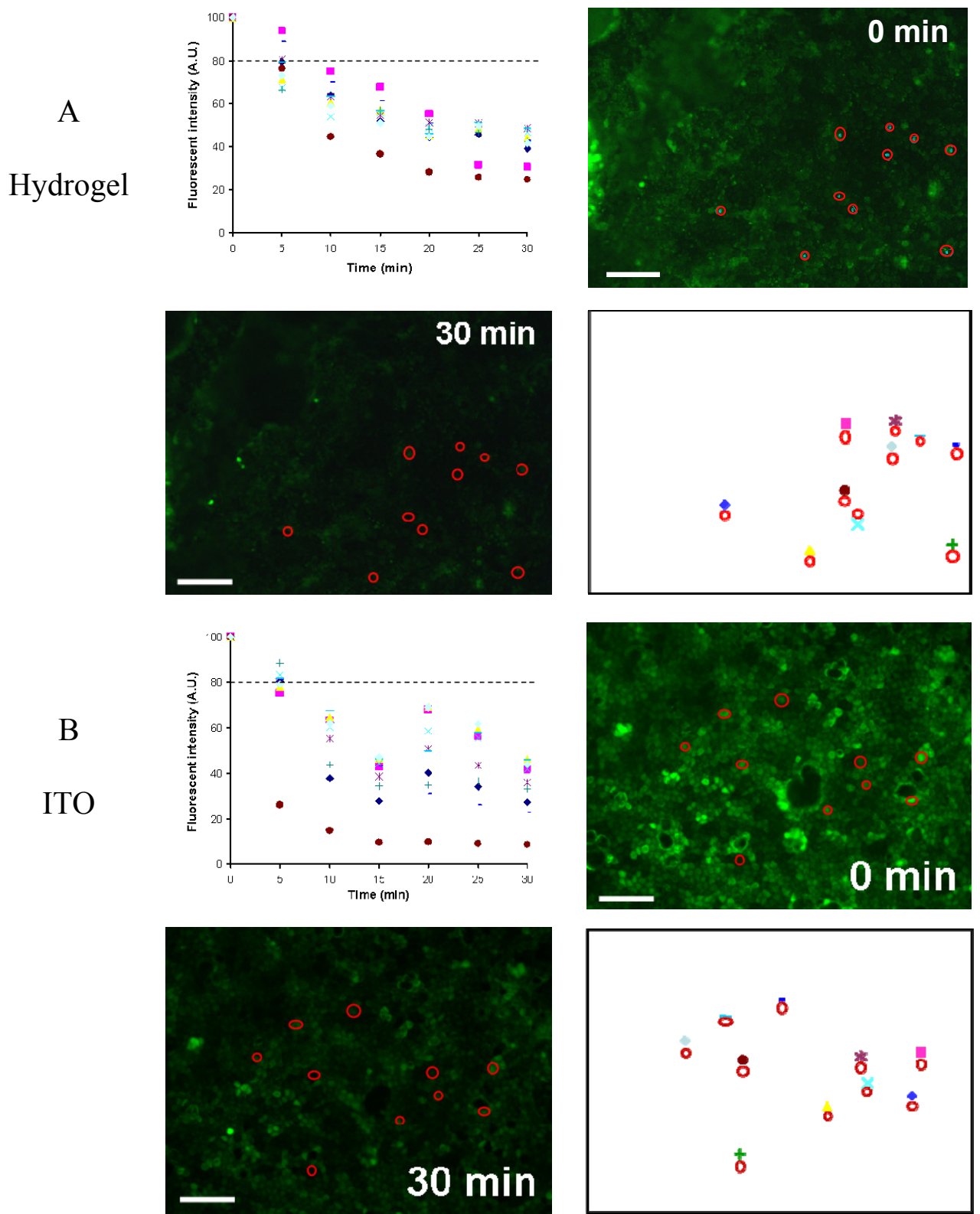

Figure 8.9. MDCK cell electroporation using a pulse amplitude of $9 \mathrm{~V}$

Quantification of the calcein release caused by electroporation in MDCK cells (8 pulses, $20 \mathrm{~ms}, 7 \mathrm{~V}$, treatment repeated every $95 \mathrm{~min}$ ) on hydrogel (A) and bare ITO (B). Pictures of the MDCK cell monolayers at 0 and $30 \mathrm{~min}$ (top right, bottom left), and graphs (top, left) showing the decrease in the fluorescence intensity for 10 individual cells. These 10 cells are indicated with red circles and the symbols representing them in the graph (bottom, right). The striped line in the graph indicates the average loss of fluorescence (20\%) caused by photobleaching. Scale bar of $50 \mu \mathrm{m}$. 
Lastly, pulses of $9 \mathrm{~V}$ are employed for the electroporation treatment, and the results of this last experiment are presented in figure 8.9 for both types of surfaces. Cells grown on hydrogel show significant decrease in their fluorescence as well (50-70\% decrease), indicating the poration of all cells in the monolayer (Figure 8.9 A). The spread in the decrease in fluorescence is lower than in the other cases, indicating a more homogeneously electroporated cell population. Cells on bare ITO give similar results as before with a decrease up to $90 \%$ (brown circle) and an average of $\sim 70 \%$ in the calcein release. For this last series of experiments, the amount of dead cells could only be measured on the hydrogel sample: 18 cells (per 260-280 cells) were found dead at the end of the experiments.

Cells grown on hydrogel are less sensitive to the electrical treatment than cells directly grown on a bare ITO surface, and for these three sets of experiments, the number of pulse trains does not seem to affect the amplitude at which electroporation is detected. Furthermore, the decrease in fluorescence due to electroporation is in most cases not linear. Lastly, the amount of dead cells increases with increasing pulse amplitude. This demonstrates that the strength of the applied field is indeed directly related to the amount of irreversibly electroporated cells. A first explanation for this difference in cell sensitivity could be a difference in the organization in their membrane depending on the surface they are grown on. However, before any conclusion can be drawn, the possible polarization of cells on both types of surfaces must be examined.

\section{Immunofluorescence}

For that purpose, immunostaining assays are performed on both types of substrates with specific markers for the apical and basolateral parts. However, ITO-coated glass substrates are not suitable for high-resolution imaging using confocal microscopy due to the thickness of the substrate and the background fluorescence created by the ITO layer. Therefore, the ITO-coated glass substrate is replaced by a thin microscope glass slide (150 $\mu \mathrm{m}$ thick), as already mentioned. Once cells have formed nice monolayers, they are fixed and stained as described in the "materials and method" section (section viii), and imaged using confocal microscopy. As a marker for the apical part, phalloidin coupled to Texas Red (red) is employed, and anti-E-cadherin (together with a secondary antibody coupled to FITC (green)) for the basolateral part (see figure 8.10). 

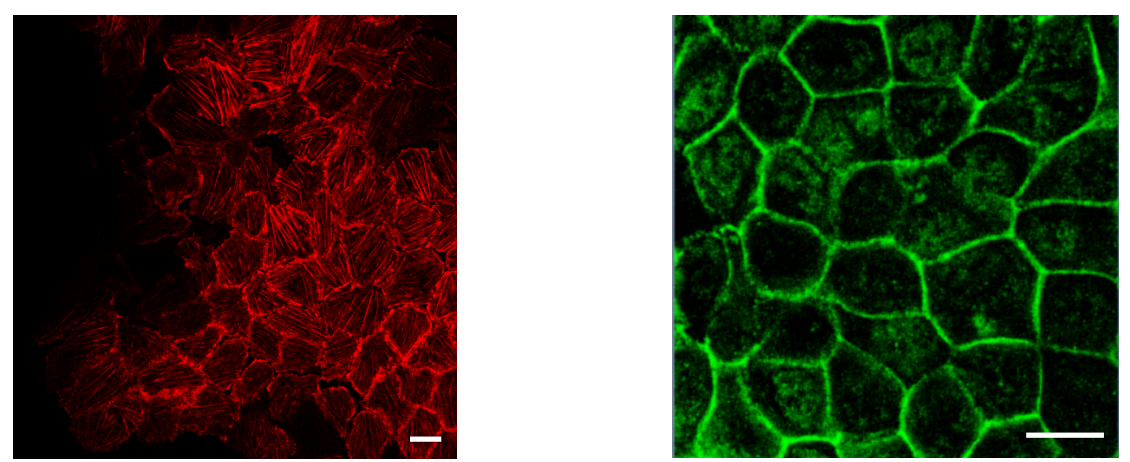

Figure 8.10. Specific staining patterns of actin (red) and E-cadherin (green).

A) Fibers of actin stained with phalloidin coupled to Texas Red (red) locate at the bottom of the cells cultured on an ITO substrate. B) E-cadherin patterns in the lateral parts of the cell membrane of a MDCK cell monolayer cultured on a glass substrate containing the hydrogel layer (staining based on anti-E-cadherin coupled to FITC (green)). Scale bar of $10 \mu \mathrm{m}$.

Figure 8.11 illustrates the staining results for both types of samples. On both surfaces, the actin staining (red) is mainly located at the bottom of the cells and shows the presence of filaments. E-cadherin is distributed between the cells, in the tight junctions formed between cells; this indicates, as expected, that E-cadherin is involved in the interactions between neighboring cells, and connects the actin cytoskeletons of neighboring cells [25]. These imaging results are in good agreement with other reports found in the literature for kidney cells. Guo et al. and Jang et al. describe similar actin fibrous patterns for kidney cells (MDCK and IMCD (inner medullary collecting duct) cells) grown on glass, hydrogel and transwell surfaces $[2,26]$. E-cadherin is also found in those works at the cell-cell contacts, and no overlap between the two dyes is found.

Surprisingly, both surfaces give the same staining pattern, and this would indicate that the cells on both surfaces display the same behavior with respect to their membrane organization. However, Jang et al. show that IMCD cells cultured on glass (non polarizing conditions) and a transwell membrane (polarizing conditions) give the same staining pattern for these two dyes, while the cell polarization is assayed with other markers for protein found in the two parts of the membranes [26]. In other words, the markers chosen here, actin and E-cadherin, reveal the organization of the cells as a monolayer, whatever their polarization level is, and do not reflect their polarization.

This is confirmed in another set of experiments where a low concentration of cells is employed so that no monolayer is formed on the substrates. Figure 8.12 shows that in that case, the two dyes overlap. The red stain still forms the same fibrous patterns for actin while E-cadherin is homogeneously distributed in the cell membrane as no cell-cell contact is established. 


\section{Material}

Actin

E-cadherin

Overlay

Side-view
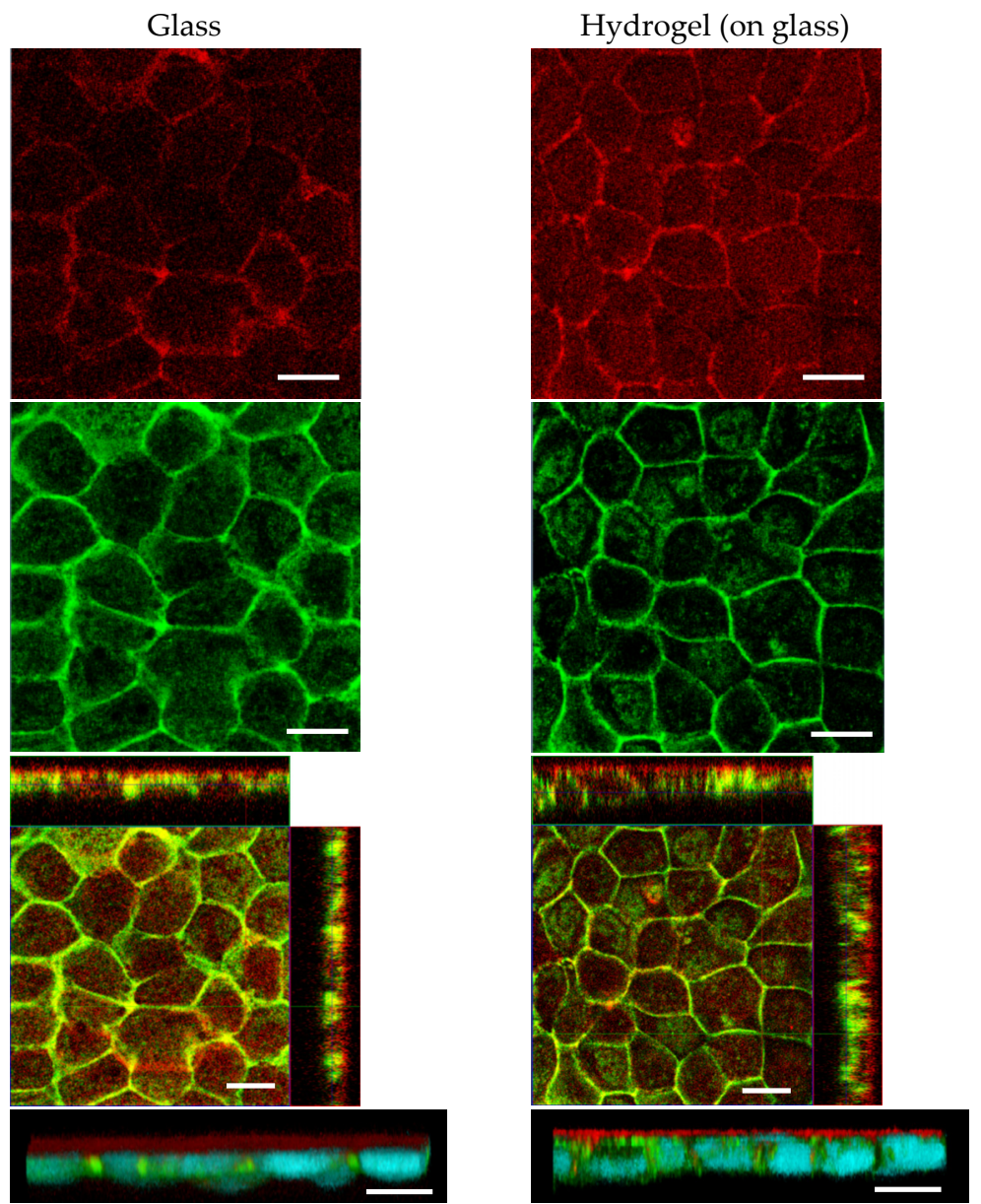

Figure 8.11. Immunofluorescence.

MDCK cell staining on glass (left) and hydrogel (right) substrates. The three first rows of pictures reveal the staining pattern observed for actin (phalloidin coupled to Texas Red), E-cadherin (anti-E-cadherin and a secondary antibody coupled to FITC) and their overlay together with orthogonal cross sections displaying the distribution of the dyes in the z-direction, respectively, recorded in the middle of the cells, while the bottom pictures present the $3 D$ reconstruction (side views) of cells stained for actin, E-cadherin and their nucleus (Hoechst). The glass and the hydrogel are located at the top of the picture whereas the buffer solution is at the bottom. Scale bar of $10 \mu \mathrm{m}$.

As a conclusion, the two markers chosen here for actin and E-cadherin do not allow for concluding on the polarization of the cells in the monolayer, and other dyes for proteins (e.g., for aquaporins) should be employed. Unfortunately, this could not be tested here.

\section{Conclusion}

In this chapter, the influence of the cell polarization on their sensitivity to an electroporation treatment is assayed. According to previous experiments described in 
chapter 6, the membrane of polarized cells present a non homogeneous sensitivity to the application of an electric field, and this directly correlates to the presence (and amount) of glycolipids in the membrane. The electroporation device consists of two PDMS wells bonded to a glass substrate covered by a thin layer of ITO, and it has been specifically designed to electroporate monolayer of cells. MDCK cells are grown on two substrates, hydrogel or bare ITO, that should induce or not cell polarization, respectively. The electroporation behaviors of cells cultured on both substrates are indeed different with cells grown on glass (ITO electrode) being less resistant to the electroporation treatment, with a difference of $2 \mathrm{~V}$. However, the reason for this difference cannot be determined here.
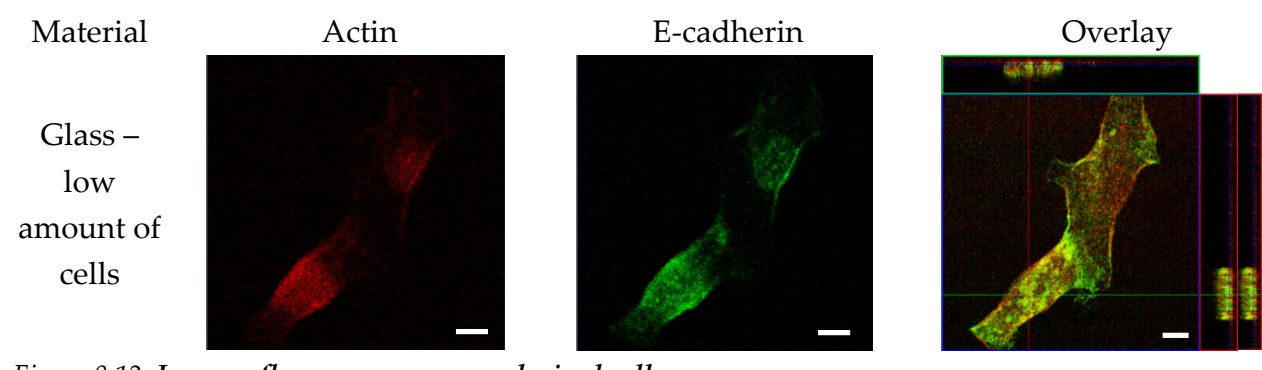

Figure 8.12. Immunofluorescence on unpolarized cells.

Immunofluorescence results on glass when the cells are cultures in a very low concentration, preventing them from forming a monolayer. The photos show the results for a layer in the middle of the cells. Left: Actin stain for the apical membrane part (phalloidin coupled to Texas Red). Middle: E-cadherin stain for the basolateral membrane part (anti-E-cadherin and secondary antibody coupled to FITC). Right: Overlay pictures together with orthogonal cross sections displaying the distribution of the dyes in the z-direction. Scale bar of $10 \mu \mathrm{m}$.

A first reason would be the polarization of the cells, or not. Polarized cells should present a lower or higher resistance to electroporation, depending whether their apical or basolateral part is first porated, while unpolarized cells should present an "in-between" behavior. The polarization of the cells was examined using immunostaining and fluorescent imaging. However, the markers chosen here do not enable to check cell polarization as both surfaces give the same staining pattern, and consequently, the relationship between polarization and cell sensitivity for electroporation cannot be established.

Other reasons could explain as well this difference in the electroporation voltage between cells grown on ITO and hydrogel that must be taken into account here.

Firstly, the presence of the hydrogel layer changes the field strength across the cell monolayer, which is accounted for by the difference in conductivity between the buffer and the hydrogel $(89.7 \pm 0.7 \mu \mathrm{S} / \mathrm{cm}$ vs. $12.3 \pm 0.7 \mathrm{mS} / \mathrm{cm}$ respectively). As a result, cells grown on a hydrogel are exposed to a slightly larger electric field than cells on ITO substrates, and this corresponds to a calculated $4 \%$ difference in resistance caused mainly by the decrease in the buffer layer height of $80 \mu \mathrm{m}$. On other aspect, the hydrogel layer has a large effect on the impedance of the device. This last point is characterized by performing impedance spectroscopy measurements (impedance / gain-phase analyzer, HP 4194A, Agilent, Santa 
Clara, CA) on wells with and without hydrogel and with and without cells. Wells with hydrogel have a much higher resistance that wells without hydrogel $(\sim 1.4 \pm 0.5 \mathrm{k} \Omega$ vs. $\sim 0.3 \pm$ $0.03 \mathrm{k} \Omega$ respectively). This difference originates again from the high conductivity of the hydrogel compared to the buffer: ions leak from the hydrogel into the buffer, greatly reducing its resistance. The impedance of the device is also affected by the presence of the cells and the resistance caused by the cells gives in theory an indication of the monolayer quality: this gives a $\sim 0.1 \mathrm{k} \Omega$ resistance increase for ITO substrates against a $\sim 0.2 \mathrm{k} \Omega$ increase for hydrogel surfaces. Although such an increase in the resistance is expected when a monolayer of cells is present, the actual values may be biased by the release of ions from the hydrogel. Both phenomena discussed in this paragraph seem to indicate that the electric field strength across the cell monolayer is much larger $(\sim 5$ times when considering the resistance of the cell monolayer equal in both cases) when a hydrogel layer is present, if the resistance of the cell-monolayer is assumed to be the same in both cases. Consequently, cells on the hydrogel would be expected to porate at a much lower potential, which is, however, not the case.

A last and most plausible explanation for this difference in electroporation voltage is the quality of the monolayer. Curiously, cells form better and more confluent monolayers on ITO than on hydrogel. On hydrogel, cells often form isolated islands and clumps separated with empty space, and this may be accounted for by the fact that the hydrogel surface is not entirely flat, forming niches where the cells preferentially grow as groups or even as tissuelike structures. However, the less homogeneous surface coverage by cells on hydrogel substrates translates in the existence of leakage pathways for the current, and consequently, a much higher electric field must be applied to actually create the desired voltage drop across the cells. This last explanation, the quality of the cell monolayer, would explain the difference in electroporation voltage observed for the two populations of cells. However, before any conclusion is drawn other aspects of the devices and in the experimentation must be better characterized, such as the influence of the presence of the hydrogel layer on the electric properties of the device and the possible polarization of the cells.

Three major problems are encountered in this series of electroporation experiments in the monolayer-based device developed here. The first issue, the quality and homogeneity of the cell monolayer, has been discussed above. An uneven surface coverage affects the electric characteristics of the device for the electroporation of the cells. If cells are cultured longer in the wells to alleviate this, cells are seen to form 3D clumps and form additional layers, which lead to the same issue. Consequently, seeding of cells on both types of substrates must be optimized to obtain fully grown single monolayers of cells for reliable electroporation experiments. Secondly, the precise effect of the hydrogel layer on the transmembrane potential should be clarified better in terms of impedance of the system and final conductivity of the buffer. A last issue concerns cell imaging during the electroporation measurements. Upon calcein release out of the cells, the buffer solution becomes fluorescent as well, creating a background noise in the fluorescence, and cells look brighter. To suppress 
the background fluorescence, cells are washed between two pulse trains and before imaging. This, however, often leads to a slight shift in the field of view and makes picture analysis more tedious to follow the same 10 cells during the whole experiment. This last effect is stronger when the extent of electroporation is larger and more calcein is released in solution. This issue could maybe be solved by the addition of a microfluidic channel connecting the well to two reservoirs that allows for the careful flushing of the buffer before a photo of the cells is taken.

This very simple monolayer-based electroporation device is highly promising to investigate the effect of the cell polarization on their response to electroporation. However, as discussed previously, refinement is necessary before reliable studies can be carried out, and the main aspect to firstly be optimized would be the protocol to prepare reproducible and homogeneous monolayers on both substrates. With this, the acquisition of more consistent results is ensured.

\section{Acknowledgements}

Hermann-Josef Kaiser kindly provided the MDCK cells. I would like to thank Tom Groothuis for the time he patiently spent with me behind the confocal microscope. Eddy de Weerd is thanked for his helpful ideas while designing the electroporation device and Hans de Boer for manufacturing the micropositioning system. I express gratitude towards Johan Bomer for fabricating the glass substrates containing the ITO layers. Paul ter Braak's help with keeping the MDCK cells alive is much appreciated. Finally, Ad Sprenkels and Loes Segerink are gratefully acknowledged for their fruitful discussion on impedance spectroscopy.

\section{References}

[1] Fuller, S. et al. (1984). Cell 38 (1), pp. 65-77.

[2] Guo, Q.S. et al. (2008). Eur. J. Cell Biol. 87 (4), pp. 251-266.

[3] Arayanarakool, R. et al. (2010). Lab Chip 10 (16), pp. 2115-2121.

[4] Bao, N. et al. (2008). Electrophoresis 29 (14), pp. 2939-2944.

[5] Bao, N. et al. (2008). Anal. Chem. 80 (20), pp. 7714-7719.

[6] Cho, Y.H. et al. In Transduces \& Eurosensors 2007, Lyon, France.

[7] Choi, Y.S. et al. (2009). Anal. Chem. 81 (9), pp. 3517-3522.

[8] Choi, Y.S. et al. (2009). Biomed. Microdevices 11 (1), pp. 151-159.

[9] He, H.Q. et al. (2007). Bioelectrochemistry 70 (2), pp. 363-368.

[10] Huang, Y. \& Rubinsky, B. (2003). Sensor. Actuat. A-Phys 104 (3), pp. 205-212.

[11] Ionescu-Zanetti, C. et al. (2008). Biomed. Microdevices 10 (1), pp. 113-116.

[12] Jain, T. et al. (2009). Lab Chip 9 (24), pp. 3557-3566.

[13] Jain, T. \& Muthuswamy, J. (2007). Biosens. Bioelectron. 22 (6), pp. 863-870.

[14] Jain, T. \& Muthuswamy, J. (2007). Lab Chip 7 (8), pp. 1004-1011.

[15] Khine, M. et al. (2005). Lab Chip 5 (1), pp. 38-43.

[16] Kim, J.A. et al. (2007). Biosens. Bioelectron. 22 (12), pp. 3273-3277.

[17] Klauke, N. et al. (2010). Anal. Chem. 82 (2), pp. 585-592. 
[18] MacQueen, L.A. et al. (2008). Bioelectrochemistry 72 (2), pp. 141-148.

[19] Miyano, N. et al. (2008). Lab Chip 8 (7), pp. 1104-1109.

[20] Valero, A. et al. (2008). Lab Chip 8 (1), pp. 62-67.

[21] Valley, J.K. et al. (2009). Lab Chip 9 (12), pp. 1714-1720.

[22] Wang, H.Y. \& Lu, C. (2006). Biotechnol. Bioeng. 95 (6), pp. 1116-1125.

[23] Wang, H.Y. \& Lu, C. (2006). Anal. Chem. 78 (14), pp. 5158-5164.

[24] Wang, H.Y. \& Lu, C. (2008). Biotechnol. Bioeng. 100 (3), pp. 579-586.

[25] Knudsen, K.A. et al. (1995). J. Cell Biol. 130 (1), pp. 67-77.

[26] Jang, K.J. \& Suh, K.Y. (2010). Lab Chip 10 (1), pp. 36-42. 


\section{Chapter 9}

\section{Conclusion and outlook}

In this final chapter, the goal of this PhD work is first recalled, the study of the process of pore formation in cell membranes in the context of electroporation, as well as the approaches adopted for this study, i.e. the use of planar model of cell membranes and miniaturized devices for membrane and whole cell electroporation. Following this, the main results of the experimental work are summarized and shortly discussed. Finally, alternative directions to carry out this research and potential improvements to the devices developed are examined. 


\section{Pore formation process}

The aim of this project is to study in more detail the process of pore formation in cell membranes during electroporation. Although electroporation is a technique that has been in use for many years, precise knowledge about the phenomena taking place in the membrane when pores form is still missing. This lack of knowledge precludes appropriate control on the process. Consequently, electroporation protocols remain sub-optimal and the poration yield low. A first strategy to increase the success rate consists of employing single cell electroporation devices where the protocol is optimized for every cell. This approach has already been investigated in our group, and previous work has led to improved electroporation yields and enabled to study the response of single cells to electroporation. However, using single cell electroporation devices still does not lead to a $100 \%$ yield and more fundamental understanding on the factors affecting pore formation is necessary. In this context, the goal of this work was to examine the influence of individual constituents of cell membranes (i.e. phospholipids, cholesterol and proteins) on their resistance to the formation of pores upon exposure to an electric field.

Two different approaches are taken to get a better understanding of which elements of a cell membrane change its strength and how. In a first step, planar membrane models (bilayer lipid membranes) are employed to study the effect of each individual component on the process of pore formation. The knowledge obtained from the model systems is coupled back to studies with whole cells, in a second step. The idea behind this is to compare the results obtained with BLMs to what happens with real cell membranes. This comparison indicates how good BLM models may be or whether they are still too simplified to describe the events occurring in a cell membrane exposed to an electric field.

Planar membrane models or BLMs are simple but highly useful models to study cell membranes. Both sides of the membrane remain accessible, for electrical as well as chemical stimuli, and the BLM composition can easily be changed. Nevertheless, in many of the reported studies employing BLMs, only a single type of phospholipid is utilized as a model for all phospholipids in the membrane, and such membranes are most likely oversimplified and not representative to what happens in a real cell membrane.

In this work, the second advantage of BLMs, i.e. the straightforward modification of their composition, is particularly exploited. The complexity in the membrane composition is progressively increased in the search for a relevant and representative membrane model to study the process of pore formation. Firstly, a single phospholipid is employed as the basic building block for the membrane, and another constituent (another phospholipid, cholesterol or a pore protein) is added. As a result, the resistance of the membranes is altered in a concentration-dependent manner and this effect can be two-fold, depending on the established molecular interactions and the intrinsic shape of the phospholipids. In a 
second stage, ternary mixtures of a glycerolipid, a sphingolipid and cholesterol are examined. These three molecules are representative for the three main classes of lipid molecules found in cell membranes. In a last step, the complexity of the membranes is again increased and BLMs whose composition mimics that of real cell membrane compositions are employed. The experimental results show that the two constituents that have the highest effect on the membrane resistance against perturbation are cholesterol and glycolipids, whereas sphingolipids have an opposite weakening effect. The reason for strengthening effect is mainly found in the packing properties of the phospholipids in the membranes. Simple mono-phospholipid membranes do not suffice to recapitulate the properties of cell membranes, and the four aforementioned constituents (glycerolipid, sphingolipid, glycolipid and cholesterol) must be considered.

Using planar BLMs is shown here as a very simple and powerful approach to study the effect of individual membrane constituents. However, the experimental set-up employed in this work could be improved, especially in terms of the used phospholipids.

The phospholipids chosen in this work are not always the most judicious and optimal ones. In the first series of experiments, a synthetic phospholipid is used as a basic building block for the membranes; this phospholipid is known to yield very stable membranes but it is however not encountered in natural mammalian membranes and originates from archeabacteria. In the second series of experiments using ternary mixtures of lipids, natural phospholipids are chosen, that are composed of mixtures of different analogues found in cell membranes. The analysis of the results is more complicated due to the higher complexity of the phospholipid mixtures and the effect of the individual phospholipid properties (such as the length of the hydrocarbon chains for instance) is difficult to distinguish. Although the fundamental studies are easier to be interpreted with single phospholipid species, the natural mixtures initially chosen better reproduce what is found in natural membranes and are subsequently more relevant in that sense. Nevertheless, if these experiments had to be done again, pure phospholipids should be preferred before moving to more complex mixtures of different phospholipid analogues, and synthetic phospholipids that do not exist in mammalian membranes should be avoided.

Besides the fundamental knowledge on the electroporation process, this research has brought additional interesting results. For instance, studies using ternary systems composed of the three main components found in natural membranes have lead to the establishment of a ternary diagram for the considered ternary system as the observed trend in the membrane response to electroporation was seen to vary as a function of the membrane phase composition. Such ternary diagrams are very useful for understanding how the different molecules in a membrane interact with each other depending on its composition. A main advantage of the newly proposed technique is its relative simplicity compared to conventional methods employed to draw such a diagram. Electroporation measurements appear subsequently to consist of a quick scanning method to study the phase behavior of 
ternary (phospho)lipid systems. Regions of interest can thereafter be studied in more detail using another more conventional method. An essential step to fully validate this new electroporation-based method is to establish the ternary diagram of a known and already published ternary system.

Secondly, in the last series of experiments, the two types of heterogeneities existing in cell membranes in an in vivo environment are examined, as well as the impact of these heterogeneities on an electroporation treatment. These heterogeneities are found either between cells in a tissue or between different parts of a cell membrane, e.g., when cells are polarized. The difference in response to the electroporation treatment observed for BLMs recapitulating the composition of cell membrane leaflets employed as models to study both types of heterogeneities indicates that the existence of these heterogeneities does indeed affect the outcome of the electroporation treatment. These data can firstly find a practical application when designing a protocol for clinical treatment using e.g., electrochemotherapy. Secondly, the similarities found in the resistance to electroporation for both types of heterogeneities are interesting for more fundamental studies; these similarities suggest that there could be a universal mechanism for cells to protect themselves to better resist external aggressions.

Lastly, electroporation experiments on BLMs recapitulating real cell membranes have brought insight on the definition of a minimal but relevant model for membrane experimentation in general. Indeed, experiments on ternary mixtures of a glycerolipid, a sphingolipid and cholesterol have revealed that these models are too simple to model cell membranes that also contain glycolipids. Good membrane models should at least be composed of multiple components; (i) a mixture of two or more glycerolipids since PC was found not to be a good model for every glycerolipid found in cell membranes, (ii) cholesterol, (iii) a sphingolipid, and (iv) a glycolipid, if applicable. Furthermore, BLMs should preferably be asymmetrical, in the same way as real cell membranes are, since the composition is very different on both sides: a glycerolipid/cholesterol mixture in the inner leaflet and a more complex mixture of four components in the outer leaflet. However, the nature of the glycerolipids to be employed to recapitulate the inner side of the membrane still needs to be investigated. The ultimate goal of this line of studies would be to discover a set of general rules to easily translate natural membrane compositions into good membrane models to study cellular membrane properties in a more reliable way.

\section{Miniaturization and microfluidics}

Another drawback of the chosen strategy to study the pore formation process is the overall throughput of the study. Conventional set-ups allow only for experimentation on one membrane at a time making systematic and extensive studies on different phospholipid mixtures tedious and time-consuming. The studies performed here can greatly benefit from the miniaturization of the system and its combination with microfluidics. This issue has been addressed by designing a miniaturized and microfluidic device that lends itself to 
multiplexing, and a microfluidic platform for BLM experimentation is developed. This sandwich system includes a Teflon foil containing a single aperture placed between two glass substrates in which the microfluidic structures are produced. Membranes are formed in this device using a novel methodology (Lipid Plug Thinning (LPT) method) that relies on the deposition of a small phospholipid plug in the aperture and its successive spontaneous thinning upon flushing of buffer in the microchannels. Membrane formation in this device is monitored optically and characterized electrically: the LPT technique yields highly reproducible and very stable membranes.

Although this device currently contains only a single aperture, its design can easily be upgraded for multiplexed studies. In such a multiplexed device, membrane formation can be parallelized and automated to allow for faster experimentation. A first key aspect towards multiplexing is to produce arrays of apertures instead of a single aperture. Microfabrication technologies lend themselves to this purpose. A second aspect is the choice of a reliable layout of the microfluidic network for multiple BLM experimentation and independent electrical measurements. For this purpose, the channels in the proposed device are disposed perpendicularly, and this can easily be extended to two series of parallel channels, with the microapertures located at their intersections, allowing for parallelized bilayer assays. However, special care should be taken in the channel design to prevent electrical crosstalk between the different BLM sites. The membrane formation technique developed in this work lends itself well to automation, and the process can be monitored both optically and electrically. Furthermore, microfluidics presents a high level of integration, and this brings the possibility to include multiple modules in the device for various functions. For instance, upstream to the BLM experimentation sites, a component can be added for on-site preparation of the lipid mixtures; this would include a gradientgenerator as well as a mixer. Thereby, a series of membranes made from different lipid mixtures can be prepared simultaneously in an automated manner. Such a platform, where the membrane composition is modified between different apertures, would be of great interest for fundamental studies on membrane properties (e.g. the establishment of a ternary diagram) or electroporation assays since a great number of parameters can be studied simultaneously.

Miniaturization and microfluidics can also benefit greatly to cell electroporation. In this work, a miniaturized device is developed for electroporation of adherent (and polarized) cells, and particularly applied for electroporating MDCK cells whose membrane compositions have been studies using BLM models. The goal of this experiment is to demonstrate, using now whole cells, the influence on the electroporation outcome of the cell polarization, as observed with BLM modeling the different parts of the cells. Using a miniaturized device to perform these experiments presents two series of advantages. Firstly, the control on the electroporation process is enhanced, and secondly, the device can be designed to work with adherent and polarized cells. A simple miniaturized electroporation 
system is developed here, where both polarized and non-polarized cells are grown as monolayers, either directly on an electrode (non polarized cells) or on an intermediate layer of hydrogel (polarized cells). With this system, the influence of cell polarization on the cell response to electroporation can be studied in a straightforward way.

A significant difference is measured in the required potential to induce electroporation between cells grown directly on the electrode and on the hydrogel. However, the precise reason for this difference could not be identified. A first option would be the polarization or not of the cells on both substrates, but this could unfortunately not be successfully validated, and other markers for the apical and basolateral parts must be examined. However, cells grown on both substrates give very different quality and confluence in cell monolayers, and this greatly affects the electrical properties of the devices. This discrepancy in monolayer quality is likely to be the main factor influencing the observed results: the hydrogel surfaces give monolayers with a poor coverage, which translates in a need for a higher electric field to induce electroporation. Consequently, the quality of the monolayers must firstly be improved for reliable experimentation on polarized/non-polarized cell monolayers.

Microfluidics offers promising and very useful experimentation platforms for the two types of experiments reported in this work, membrane experimentation and electroporation applications. They offer higher control on the experimentation as well as a route to multiplexed experimentation sites. The first class of devices, for membrane experimentation, can for instance not only benefit for high-throughput screening of drugs, but also for more fundamental studies on cell membrane properties. For the second application, microfluidic devices can easily be designed for single cell electroporation, high-throughput electroporation or monolayer-based electroporation (as is described in Appendix A). This last direction has been targeted here, and in spite of the shortcomings we encountered, we do believe that such a miniaturized and microfluidic tool will bring valuable knowledge on the influence of cell membrane properties and their polarization on their response to an electroporation-based treatment. In general, new knowledge gained for both types of experiments will allow medical and biological experiments to be carried out more successfully, and further stimulate the use of this valuable technique. 
Appendix A

Review miniaturized electroporation devices 


\section{Introduction}

\section{A. Electroporation technique}

Electroporation is a technique employed to transiently permeabilize cell membranes in the purpose most of the time of introducing foreign material into the cell. Electroporation relies on the application of short pulses of a high electric field on the cell membrane, and a comprehensive review on this technique can be found chapter 2 . Briefly, as soon as the transmembrane potential exceeds a certain threshold value of $0.2-1 \mathrm{~V}$ [1-2], conducting pores are formed in the membrane. Depending on a number of parameters related to the electrical treatment and the cells, three outcomes are found for this process, as illustrated in figure A.1: (i) no detectable change takes place in the membrane (absence of pores or too small pores); (ii) the cell is lysed or irreversibly porated) [3-4]; or (iii) pore formation remains transient and the cell is reversibly porated. In this last scenario, a short-lived rearrangement in the molecular structure of the membrane leads to the formation of pores and consequently to a substantial increase in the cell permeability to foreign entities [2,5-7]. The membrane starts to recover after termination of the pulse, ensuring cell survival and enabling the uptake by cells of any molecule added in the solution [6, 8-10]. As explained in more detail in chapter 2, molecules are transported across the membrane by different mechanisms depending on their properties. The uptake of small molecules is purely based on diffusion while large molecules, such as DNA and proteins however, require more complex transportation mechanisms. These molecules interact with the membranes and are possibly transiently stuck in the pores in the membrane (protruding on both sides) before being electrophoretically pulled through [11].

\section{B. The conventional technique and its limitations}

Conventionally, electroporation is performed in a cuvette on a cell suspension (typically $10^{4}-10^{6}$ cells or several hundreds of $\mu \mathrm{L}$ to a few $\mathrm{mL}$ ), and a high voltage of several $\mathrm{kV} / \mathrm{cm}$ is applied on the cuvette. This conventional approach presents two major drawbacks. Firstly, the success rate of the technique remains low, with only $40-70 \%$ of the cells being viably electroporated [12], the largest part of cells being unaffected and a small amount of cells dying. This low poration yield is partly accounted for by "historical" reasons. Originally, electroporation has been developed to transfect bacteria, and only one transfected and surviving cell is sufficient to create a functional colony; in other words, the success rate of the process was not a major point of interest [13]. Only when the transfection experiments started to focus on mammalian cells, cell survival became an issue.

Several factors explain the low poration yield. Cell populations are heterogeneous in size, shape and membrane properties. In a cuvette, cells are oriented randomly with respect of the electric field, so that they are also exposed to a different electrical treatment. A second set of drawbacks originate from the use of a high voltage supply: undesired chemicals are produced on the electrodes; those are toxic to the cells and can affect the electroporation 
outcome.

On other aspects, this conventional cuvette setting is not suitable for the electrical treatment of adherent and rare cells. Adherent cells must firstly undergo a (bio)chemical treatment to be placed in solution; this can cause damage to the cell membrane and consequently affect the response of the cell to the applied electric field and lower their survival rate. Rare cells are generally available in limited quantities compared to the $10^{4}-10^{6}$ cells handled in the cuvette.

A promising alternative approach to alleviate the aforementioned issues and enhance the control over the electroporation process consists of using miniaturized or microfluidic devices, as discussed in the next paragraph.

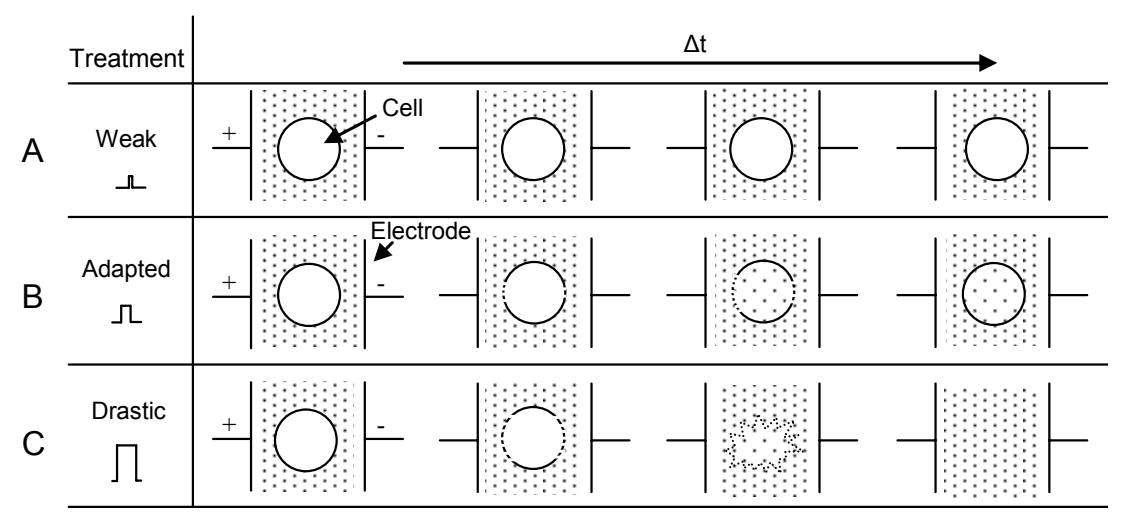

Figure A.1 Schematic representation of the three possible scenarios of electroporation.

A) Weak treatment: the transmembrane potential does not reach its critical value. Subsequently, the cell is not permeabilized or pores are too small to be detected. B) Intermediate treatment: the transmembrane potential does reach its critical value and a finite amount of pores is created in the membrane. The cell membrane reseals and electroporation is successful (reversible electroporation). C) Drastic treatment: many and large pores are formed in the membrane that do not close after termination of the applied electric field. The cell dies (irreversible electroporation).

\section{Miniaturization of the electroporation devices}

Miniaturized and microfluidic devices have been introduced in the 90's for analysis purposes. Since their introduction in the field of life sciences, those devices have seen their applications greatly diversified and they are now considered as promising tools to scale down cell experimentation. A short introduction on microfluidic devices and their main advantages is provided in chapter 3 , section IIIAi.

In particular, for the technique of electroporation, microfluidic and miniaturized devices bring a number of attractive features. As the experimentation is easily scaled down to a limited number of cells, electroporation protocols can be developed at the single cell level, with parameters separately optimized for every cell. This higher control on the 
treatment leads in theory to a $100 \%$ electroporation yield. On other aspects, due to the small size of the device, the distance between the electroporation electrodes can be decreased down to a few tenths of micrometers. In that case, an applied potential of a few volts is sufficient to generate the $\mathrm{kV} / \mathrm{m}$ field strength required for the process of electroporation. In addition to this, microstructures can be implemented in the device to shape the electric field and create hot spots of electric field. The protocol obviously becomes even milder, and this suppresses risks of undesired irreversible cell damage. Last, miniaturized devices are adequate for the treatment of rare cells which are available in a reduced quantity and for the study of the heterogeneity of cell populations by examining the variations in the cell sensitivity to the application of an electric field.

In the next sections of this review, an overview is given of existing miniaturized and microfluidic electroporation devices. They are classified into three categories depending on their cell handling properties; single cell trapping devices, flow-through devices and monolayer-based devices. For each of the three classes, the different approaches and specifications are discussed together with the fields of application for these systems and their limitations. Before this, a short introduction to the electroporation experiments is provided, i.e. on how electroporation is characterized and demonstrated in microdevices. In the last part, the complementary of the three miniaturized approaches for electroporation are discussed, together with some ideas about further developments and future applications.

\section{Miniaturized and microfluidic electroporation devices}

\section{A. Electroporation experimentation}

For all miniaturized and microfluidic devices discussed below, the experimental approach is the same to detect pore formation in the cell membrane and assess the performance of the devices. Two main techniques dominate for monitoring pore formation: optical (fluorescent) measurements or, more rarely, electrical measurements.

Optical measurements proceed in two steps, and employ two types of fluorescent probes. In a first step, the experimental parameters are adjusted until cell poration is seen. For this step, fluorescent dyes are typically employed and their uptake by or release out of the cells monitored [14-34]. These dyes are viability markers that indicate the loss of integrity of the cell membrane, or in the present case the existence of pores in the membrane. Once correct electroporation parameters have been found, gene transfection experiments are performed, and typically, the gene coding for the green fluorescent protein (GFP) is employed for proof-of-principle experiments as GFP expression is easily visualized [20, 22, $24,26,28,30,34-42]$. Cells which do produce the protein have not only being porated and survived the treatment, but they are also functioning properly as they can produce proteins.

Alternatively, pore formation is detected electrically, by measuring the increase in the membrane conductance [25, 43-46]. However, a pre-requisite for these measurements is that cells are tightly trapped in dedicated microstructures between electrodes So that a G $\Omega$-seal 
is formed between the cell and the trap reducing the current leakage and increasing the sensitivity. In that case, the change in conduction is large enough to be measured.

Interestingly, while the optical approach is easier to implement in a device, it remains invasive. In contrary, the electrical approach appears to be more attractive for ultimately monitor the process of pore formation in commercial devices, as it is a non-invasive approach. Furthermore, pore closure can be monitored by the decrease in conductance over time, giving information about cell survival.

\section{B. (Single) cell trapping devices}

A first dream brought by microfluidics to cell biologists was the possibility to carry our experimentation at the single cell level. In this context, the first miniaturized cell electroporation devices were developed to design new protocols on individual cells, and these are (single) cell trapping devices.

\section{(i) Brief description of cell trapping devices}

In trapping devices, cells are isolated in dedicated microstructures between two (micro)electrodes, and a single device can include from one to two hundreds (individual) trapping sites [43, 47-48]. The trapping structures can be classified into two groups, (i) orifices located under the cells or planar trapping sites and (ii) narrow slits or lateral trapping sites, as detailed below. All structures present a characteristic dimension of 2-3 $\mu \mathrm{m}$, which is several times smaller than the cell diameter. Interestingly, these trapping structures also enable to shape the electric field and create hot spots across the cells $[25,30]$. The electrodes employed to apply the electric field are sometimes external and placed in the access reservoirs [21, 25, 46] while most reports describe integrated electrodes [30, 36, 43-45, 48-51]. In the former case, the electrodes are mostly common, and no real single cell electroporation is possible if the device include multiple traps. In the latter case, the electrodes are placed in close vicinity to each trapping sites. As explained above, as cells are porated individually, this approach is highly attractive to derive all the electroporation parameters for every cell and to eventually give a $100 \%$ (viable) poration yield.

Typically, cell electroporation proceeds as follows in a cell trapping device. The cell suspension is injected in the device while a negative pressure is created across the trapping sites to immobilize the cells in the dedicated structures. Once all traps are filled an electric field is applied, and cells are electroporated, simultaneously (common electrodes) or individually and successively (individual electrodes). In the former case [48], an average electroporation signal is chosen to maximize the poration yield, while in the latter case [21, $25,30,36,43-46,49,52]$ the pulse parameters are adjusted and optimized as a function of the cell properties. A key-step in this protocol is the trapping of cells, as this affects the outcome of the treatment. A too high negative pressure is likely to damage the cell, and in turn increase the cell vulnerability and the associated risks for cell death. On the contrary, if this force is too low, or, respectively, the trapping structure too large, the cell may easily escape the trap. A leakage pathway is created in the trap so that a higher potential must be applied 
for cell poration. This leakage pathway also obviously prevents the electrical detection of pore formation.

\section{(ii) Two classes of cell trapping structures}

The most important features in cell trapping devices are the trapping sites, and two different types of features (planar or lateral) have been described to isolate individual cells. In both cases the trapping sites separate the channel or chamber where cells are introduced and a second fluidic structure employed to generate a negative pressure across the trapping sites. In a first option, the trapping structures are located at the bottom of the microfluidic chamber or channel [36, 43-44,46,48-52] while the second option consists of employing lateral trapping sites $[21,25,30,45]$. Both options are discussed successively in the coming section.

\section{Planar trapping}

The first microfluidic electroporation device described by Huang and Rubinsky employs a (single) planar trapping site [43-44]. The device is composed of two silicon chambers separated by a horizontal $1-\mu \mathrm{m}$ thick silicon nitride membrane containing a $2-10$ $\mu \mathrm{m}$ diameter orifice (see figure A.2A). The two electroporation electrodes are integrated in the device; they consist of $n+$ polysilicon membranes placed in the two silicon chambers and separated by $900 \mu \mathrm{m}$. The semi-conductive and translucent properties of this material are suitable for both electrical and optical monitoring of cell trapping and poration. Trapping of cells in the orifice can be observed and leads to a great reduction in the leakage current upon sealing of the cell on the substrate. Yet, electroporation is only detected electrically by the increase in the conductance across the orifice. Two different signals are compared for cell electroporation: when a voltage ramp $(50 \mathrm{~V} / \mathrm{s})$ is employed, pore formation occurs at potentials around $20 \mathrm{~V}$ while when single pulses of $60 \mathrm{~ms}$ are applied the cells are permeabilized around $40 \mathrm{~V}$.

A large number of trapping devices are based on the same principle as this first system, with two large chambers separated by a membrane than contains one or multiple trapping orifices. The substrate containing the orifice(s) mostly consists of a silicon nitride membrane [43-44, 46, 49-53] although polymers such as Kapton and PDMS have been employed for the same purpose [36, 48]. Coating of this substrate with parylene [52], antibodies on gold or poly-L-lysine [50-51] has been reported to enhance the electrical properties of the devices and the seal between the cell and the substrate. The electrodes employed to apply the electric field across the cell(s) are either external [43-44, 48, 52] or integrated in the vicinity of the trapping sites [20,36, 50-51].

A main advantage of this planar approach is its amenability to large-scale trapping with the implementation of 2D arrays [48]. However, in these planar trapping devices, no single cell electroporation is reported on a cell population: either only one cell per experiment is electroporated if the device includes one single trapping site, or all cells are electroporated simultaneously, in case of a multiplexed device. This limitation has been 
addressed by Tixier and Le Pioufle [50-51] who have proposed a device where cells are trapped in an array of cavities that integrate both a microhole and individually addressable microelectrodes. With this design, single cell electroporation is envisioned on a large number of cells, although it has not been really demonstrated. On other aspects, the optical detection may be hindered by the multi-layer structure of the devices.

\section{Lateral trapping (multiplexed designs)}

The second category of trapping devices employs lateral trapping sites which are positioned between two "parallel" microchannels. This design lends itself better to electrical decoupling of the traps and consequently to multiplexed single cell electroporation, as long as the trapping structures and channels are electrically insulated from each other.

In this category, the first device is composed of a large circular chamber from which fourteen $50 \mu \mathrm{m}$-high side channels are radiating (see figure A.2B) [25, 54]. Channels on the chamber side are designed to be very small ( $4 \mu \mathrm{m}$ width and $3.1 \mu \mathrm{m}$ height) for tight sealing of the cells in the traps. All structures are made from PDMS and bonded to a glass slide, and $\mathrm{Ag} / \mathrm{AgCl}$ electrodes are inserted in the large central reservoir and the outlets of the side channels for individual cell treatment. Cells are successfully electroporated with a very mild treatment, $6.5-\mathrm{ms}$ pulses of $\sim 0.76 \mathrm{~V}$ amplitude, and this is simultaneously monitored electrically and optically In a further study, a feedback loop is implemented to enhance cell survival; as poration is detected electrically, the application of the treatment is stopped as soon as a change in the membrane conductance is detected $[45,55]$. In parallel, this device is integrated in a 96-well plate for higher poration throughput [45]. In a second refinement, the electrodes are also employed to assist the introduction of foreign substances into the cells using electrophoresis [21], and thereby increase the transfection yield.

An alternative device has been proposed by our group (see figure A.2C) [56]; it contains two parallel and $15-\mu \mathrm{m}$ high microchannels fabricated in a silicon substrate and separated by a series of nine $4-\mu \mathrm{m}$ wide microslits (also $15 \mu \mathrm{m}$ height) used for cell trapping. In one channel (50 $\mu \mathrm{m}$ wide), the cell suspension is introduced while the second channel $(20 \mu \mathrm{m}$ wide) is employed for applying a negative pressure. The electrodes are integrated in the device and sputtered on the glass lid of the devices; one electrode is located above each microslit and a common electrode is found behind the trapping sites. Cells are individually porated and transfected with pulses of $6 \mathrm{~ms}$ and $2 \mathrm{~V}$ amplitude, with an overall success rate of $>75 \%$. Interestingly, this device has been applied for examining intracellular signaling pathways in individual cells. For that purpose, a gene coding for a construct protein between GFP and a signaling protein (ERK1) is introduced in the cell [30]. Real-time imaging of the protein position upon cell stimulation with specific growth factors enables to follow the activation -or not- of signaling pathways that involve this specific protein. 


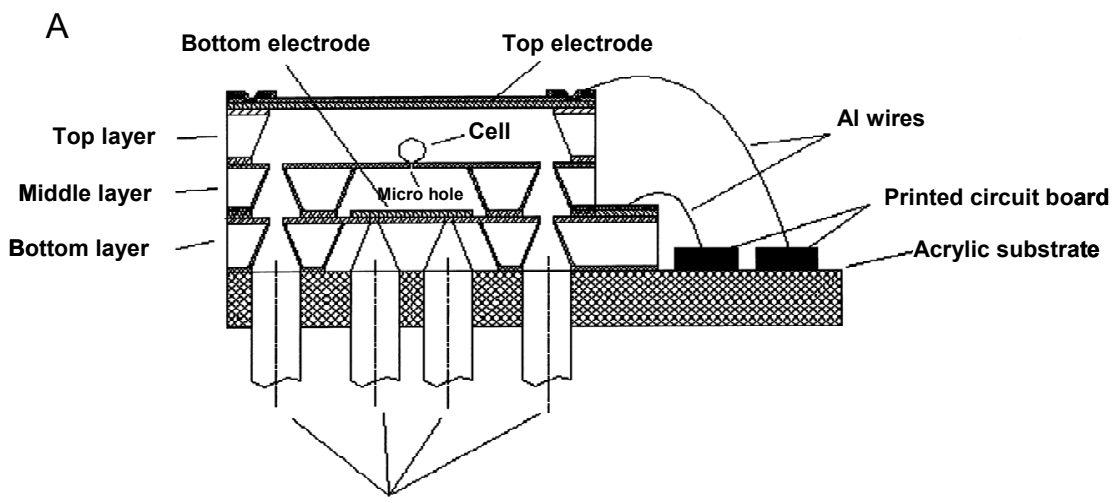

Fluid inlets/outlets
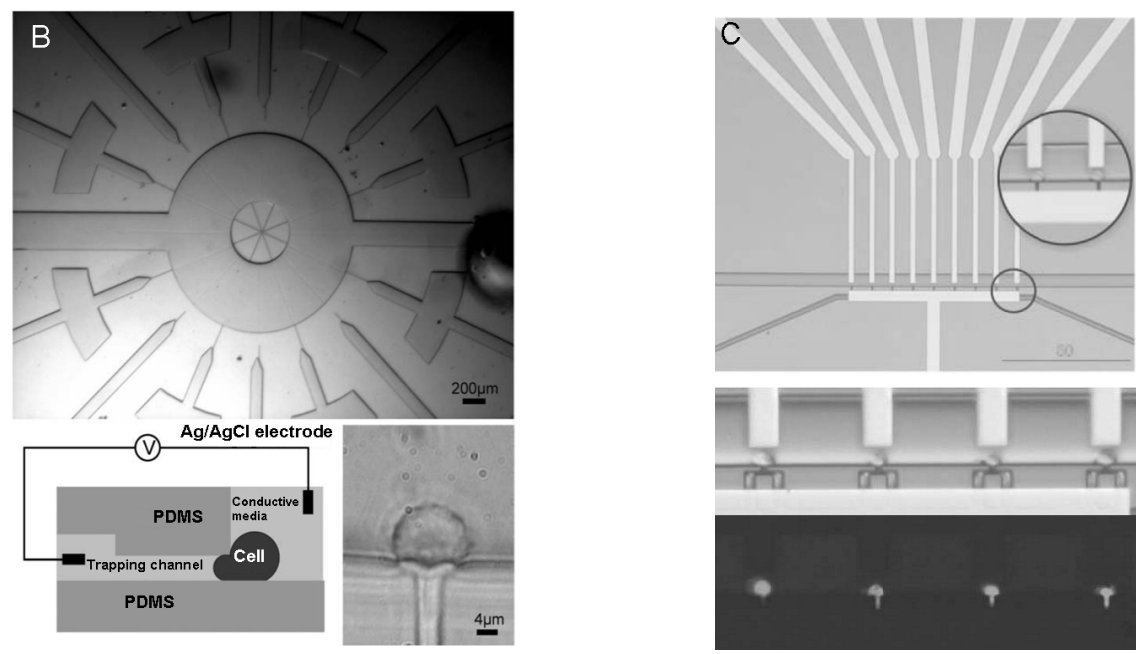

Figure A.2. Cell trapping devices

A) Three layer single cell electroporation device consisting of two microfluidic chambers in silicon, separated by a $1-\mu \mathrm{m}$ thick silicon nitride membrane containing an aperture $(2-10 \mu \mathrm{m}$ in diameter) on which the cell is trapped before electroporation. The top and bottom electrodes are formed by a transparent $n+$ polysilicon membrane [44]. B) Top: Top view of a PDMS device for lateral cell trapping containing 14 individual trapping sites. Bottom left: Schematic cross-section of the chip displaying a trapped cell and the two electrodes employed to generate the required field. The trapped cell is pulled into the small opening due to the applied suction force. Bottom right: Photograph of a trapped cell [25]. C) Top: Top view of a silicon-glass microfluidic single cell electroporation device. Two microfluidic channels are connected by nine cell traps where cells are trapped with the help of a suction force. The electroporation signal is individually applied to each cell with the nine stimulation electrodes located above the traps and the common ground electrode placed below the traps. The zoom-in shows a picture of trapped single cells between the electrodes; Bottom: Bright field and fluorescent microscopy images of four trapped cells (C2C12) before and 24h after transfection with EGFP-N1 respectively. The transfection resulted in a 75\% success rate [30]. 


\section{(iii) Advantages and limitations of (single) cell trapping devices}

A main advantage of this first category of miniaturized electroporation devices is the use of milder electrical parameters and the higher control on the poration process, which bring enhanced yields in cellular treatment. Since the electrodes are very close to each other and the cells can be tightly trapped, the applied potentials become very low compared to conventional systems, ranging from $20 \mathrm{~V}$ [43-44] down to $>1 \mathrm{~V}$ [25], and the success rate for the treatment can go up to $100 \%$ [21, 45]. On other aspects, plasmids can be actively brought into the cells by electrophoresis, which is not possible in bulk electroporation [21]. On other aspects, single cell electroporation is possible in the devices, when the device includes a single trap, or when the different electroporation sites are electrically decoupled. The electric field can be tuned to match the cell properties, which further increases the poration yield. Finally, the electric field distribution across the trapped cell can be modeled; the cells are most of the time considered as spheres with only a small protrusion into the channels [25, 56] and their position in the electric field is well-defined. These modeling aspects also contribute to the better control on the poration treatment. All these advantages make these (single) cell trapping devices the best suited for the treatment of rare cells.

An important drawback of these designs it their level of complexity: they include multiple channels or chambers with micrometer-sized trapping structures, and sometimes integrated electrodes, and this mostly translates into sophisticated fabrication processes. However, the main limitation of (single) trapping devices is their treatment capacity as they present a small number of traps, and this, in turn, gives a low cell treatment throughput.

\section{B. (Single cell) flow-through devices}

One way to increase the throughput of the devices is to use a flow-through device where cells flow in a microchannel and are treated successively. Most of the time, the microchannel comprise a local region of high electric field, to which the cells are exposed when they flow in the channel, and they subsequently become porated. Cells can also be collected at the outlet of the device, for further study, for instance. With this approach, cells are still treated individually but at the level of a (larger) cell population.

Two classes of devices are found in this flow-through category. The first devices resemble the (single) cell trapping device in that a single orifice is used as a cell trap to position the cells between the electrodes and as a hot-spot for the electric field. After the cells have been exposed to the electric treatment, they are released from the orifice and another cell is subsequently immobilized and porated [20,31]. The second class of devices does not involve any trapping step; cells flow in a microchannel which contains geometric constrictions to create areas of high electric field. Both options are discussed separately in the next section. 


\section{(i) Constriction of the electric field}

\section{Cell trapping}

Two examples only are found in the literature in this category where cells are successively trapped, porated and released. The first device is an extension to the planar cell trapping design proposed by Huang and Rubinski (see figure A.3A) [43-44, 49], where the top silicon chamber is replaced by a SU-8 microchannel. Two sets of electrodes are integrated in the device, one set to detect the presence of a cell in the vicinity of the orifice and one for the actual electroporation treatment after trapping of the cell [20]. Individual cells flowing in a $30-\mu \mathrm{m}$ wide section of the channel are guided towards the small orifice, detected thanks to the first set of electrodes and porated using the second set of electrodes. Finally, a positive pressure is applied to release the cell and let the orifice free for the next cell. A $100 \%$ electroporation yield is achieved with this flow-through protocol and an electroporation signal consisting of multiple pulses of $10 \mathrm{~V}$ and $100 \mathrm{~ms}$. However, the actual throughput of the device or the total time to trap and porate a single cell is not reported.

Alternatively, dielectrophoresis is employed to trap individual cells in a microchannel between two electrodes [31]. Valley et al. employ a photosensitive layer deposited on two ITO electrodes placed at the bottom and top of a microfluidic channel. This material presents different electrical properties depending whether it is illuminated or not. Without light, it acts as an insulator but when exposed to light, it becomes conductive. Subsequently, activation of this material through a photomask leads to the creation of a series of hot spots of electric field in the exposed areas. The same electrodes or activated areas are employed for both cell trapping by dielectrophoresis $(1.1 \mathrm{~V}$ at $10 \mathrm{kHz})$ and cell poration $(8-13 \mathrm{~V}$ at 10 $\mathrm{kHz}$ for $5 \mathrm{~s}$ ). Another attractive feature of this device is its amenability to multiplexing as a large number of individual traps can be created simultaneously, although single cell poration is not possible. However, although the performance of the device has been illustrated for trapping and electroporating four cells simultaneously, its throughput is not mentioned, nor its success rate.

\section{Formation of high electric field areas}

The second category of devices relies on the creation of hot spots of the electric field in a simple channel, and this can be achieved using several approaches. One method simply consists of placing two electrodes at a short distance from each other inside a channel [38, 40-41, 57-58]. For instance, two thin gold film have been placed on the top and bottom of a PMMA channel (5 mm wide; $0.2 \mathrm{~mm}$ high), and DC pulses are applied on the resulting electrodes (10 V, ms-range duration, $5-10 \mathrm{~Hz}$ frequency). In this configuration, the electroporation treatment to which cells are exposed depends on the flow-rate, and so do the success yield and cell survival rate. Increasing the flow speed of the cells through the device and decreasing the pulse length both result in a higher success ratio (up to $90 \%$ ).

In terms of electrode fabrication and electrical connections, a design with both electrodes on the same substrate is preferred, while they give inhomogeneous electric field 
distributions [16, 41, 59]. Another alternative relies on the use of 3D electrodes [16, 59], and for instance, copper pillars have been shown to produce better results in terms of uniformity and strength of the electric field, for cell poration [59]. With this device, no electroporation is demonstrated, but the lysis of cells by irreversible electroporation.

Another option consists of locally enhancing the electric field at the intersection of two microchannels [37, 42,60], with electrodes positioned in the side channel(s) while cells are flowing as a single cell file in the main channel. Cells are consequently shortly exposed to the electric field and the voltage drop is higher across the cells. In a first example, the side channels are directly connected to the cell channel and the cell solution is focused as a single cell line between two flows of a high ionic solution to create a larger potential drop across the cell solution upon application of a low DC voltage $(1.5 \mathrm{~V})$ in the side channel as shown in see figure A.3B [42]. This approach gives a throughput higher than $10^{4} \mathrm{cells} / \mathrm{min}$, with $90 \%$ of the cells remaining viable after the treatment. In an alternative approach, the channel is made narrower in the vicinity of the intersection so that cells flow successively in the constricted region of a higher electric field [40]. In this example, a fluidic junction (e.g., a polyelectric salt bridge [37]) is created between the main and the side channels to avoid direct exposure of the cells to the electrodes, and the electrodes are inserted in a hypertonic solution (see figure A.3C). A small DC voltage (7-15 V) is employed for electroporation, and after tuning the flow-rate, a success rate up to $72 \%$ for difficult-to-transfect cells is obtained with a throughput of $10^{5}$ cells/min.

Alternatively, the main channel presents one or several constricted areas where the electric field is concentrated [14-15, 32-34, 61], and electrodes are inserted in the fluidic reservoirs of the devices. The length of the constricted area and the flow-rate define the pulse length, whereas the channel width correlates with the strength of the electric field. Wang et al. developed a PDMS device that exploits this simple principle. A $213-\mu \mathrm{m}$ wide channel contains a 2-mm long portion with a width of $33 \mu \mathrm{m}$ to give a local 6.5-fold enhancement of the electric field. Cell viability after successful electroporation remain higher than $90 \%$ after optimization of both the flow rate $(1.2-2.7 \mu \mathrm{L} / \mathrm{min})$ and the electric field parameters (41-110 V DC), and the device gives a throughput of $1.8 \times 10^{3}$ cells $/ \mathrm{min}$. Furthermore, with the same design, multiple pulses can be applied by implementing a series of constrictions in a single channel (see figure A.3D) [34]. The same principle has been exploited in the so-called technique of electroporative flow cytometry where pore formation is employed for cell analysis and release of material out of the cells [14-15]. This principle has notably been illustrated to investigate signaling pathways in single cells [14]. 

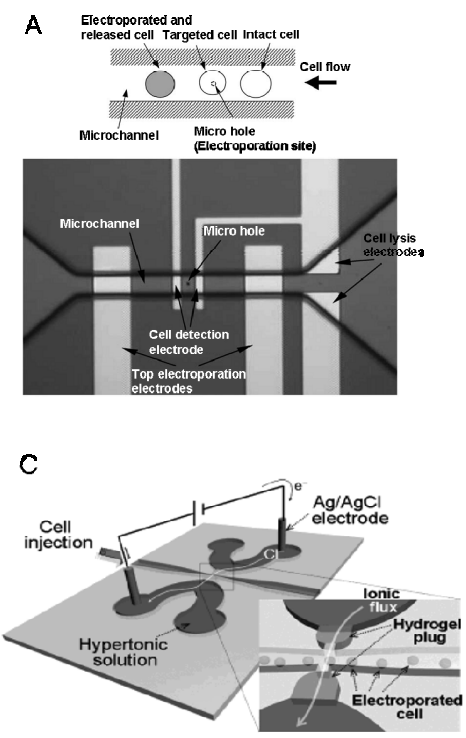

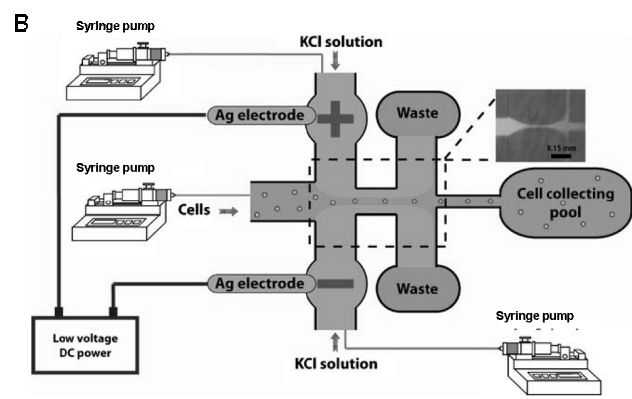

D
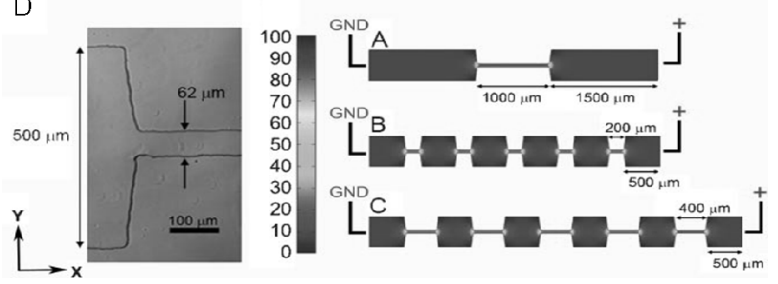

Figure A.3. Flow through devices

A) Top: Operational principle of a flow-through electroporation device. Cells flow through the channel, and are trapped on to of the aperture by the applied suction force. After they are electroporated, the treated cell is released and collected at the end of the channel. Bottom: Top view of the device indicating the aperture, microfluidic channel, and integrated electrodes employed for the detection of cells flowing through the channel and for applying the electroporation signal [20]. B) Schematic of the hydrodynamic focusing electroporation device. Cells are focused as a single line before they flow through a region of high electric field. Inset: Fluorescence images captured during an experiment showing the principle of hydrodynamic focusing [42]. C) Schematic drawing of the electroporation chip including hydrogel plugs that function as salt bridges to form an integrated electrode junction in the channel. Cells are exposed to the electric field and porated in the region between the salt bridges [37]. D) Right: Microscopy image of the decrease in channel width in the device employing channel constrictions to create a region of high electric field to electroporate the cells. This device employs the principle that the electric field scales inversely with the channel width, i.e. when a region in the microfluidic channel is smaller, the electric field across it is higher. Left: Simulation of the electric field strength in the channel constriction system. Cells are exposed to one or multiple high electric field regions, depending on the design employed [34].

\section{(ii) Advantages and characteristics}

The main interest of these flow-through devices is the throughput in cell treatment which reaches values of up to $10^{5}$ cells/min, which is comparable to what is obtained with flow cytometry. As for the first category of devices, cells are still treated individually, and the efficiency rate is as high as for the (single) cell trapping devices. However, parameters of the electrical treatment have not been yet optimized at the single cell level and the whole population is exposed to the same treatment. Still, this configuration can be developed further to couple cell analysis to cell electroporation, prior or after the electrical treatment. For instance, the cell size and morphology can first be characterized to better tune the electroporation parameters, and the cellular content analyzed after electroporation. A small 
drawback in these devices is the difficulty to precisely model the electric field distribution across the cells, as the latter keep a certain degree of freedom in their position and orientation with respect to the electrodes. Lastly, this flow-through approach is still not suitable for the treatment of both adherent and rare cells, for the reasons evoked earlier.

\section{C. "Monolayer-based" devices}

The third class of devices is the "monolayer"-based devices which are specifically meant for adherent cells, and the electroporation treatment is operated in situ in a more natural environment for those latter cells. Cells are grown directly on one electrode which is subsequently employed for the electroporation treatment, or between the two electrodes. Furthermore, in this configuration, cells are stretched on the surface, so that their membrane surface-to-volume ratio is increased, and this translates into the formation of more pores and increases the intracellular delivery. Interestingly, in this configuration, the entities to be introduced into the cells can be concentrated in the vicinity of the monolayer, and this promotes their uptake by cells after pore formation.

\section{(i) Electrode design}

One of the most important features in monolayer-based devices is the electrodes [17-19, 23-24, 27-29, 35, 39, 62-63], and different designs have been proposed, and the following designs are successively presented here: external electrodes, planar electrodes, interdigitated electrodes, electrode arrays (MEA) and 3D electrodes.

\section{External electrodes}

One device makes use of standard external electrodes [26]. Cells are grown as monolayers in a series of channels, all connected to the same reservoirs, where electrodes are inserted. The length of the channels is varied to give different electric field strengths upon application of the electroporation signal (3-7 pulses of 7-10 ms length of 1.3-kV amplitude) to give transfection rates up to more than $90 \%$ for shorter channels.

\section{Planar electrodes}

A second design of electrodes found in monolayer-based devices consists of two planar electrodes positioned opposite to each other [17-18, 27], and between which cells are grown [17-18, 27]. A first very simple device in this category consists of a 48-well array [22] bonded on a transparent ITO electrode, the second electrode being placed in solution. Cells are grown on the ITO electrode, and simultaneously electroporated with $>50 \%$ efficiency, when a signal ( $1-8$ pulses, length of $0.1-100 \mathrm{~ms}$ and amplitude of $5-80 \mathrm{~V})$ is applied. A main application of the device is the parallel loading of cells with different plasmids or siRNAs. The use of a miniaturized device for this purpose not only reduces the experimentation time, but also the costs, as less reagents are required.

A second interesting device in this category reproduces the electrode configuration(s) employed for in vivo electrochemotherapy-based treatment of breast cancer. The device integrates two [18] or six [17] gold electrodes patterned on a glass substrate (see figure A.4A) 
and cells are grown on it, in the area defined by a PDMS well. Prior to the electrical treatment (108-150 V pulses), the anti-cancer drug bleomycin is added in the well [18]. The six electrode design gives a success rate of up to $100 \%$, whereas with the two electrode design, the field distribution is less homogeneous, and the success rate lower. Such a device finds application in assessing the geometry of electrodes to be used for in vivo electrochemotherapy [13].

\section{Interdigitated electrodes}

Interdigitated electrodes are capable to generate very high electric field in the vicinity of their surface, and are for this reason highly attractive for the poration of adherent cells directly grown on them [64]. For instance, primary cells have been cultured on a glass substrate covered with interdigitated electrodes (see figure A.4B) [39, 64]. Electroporation is observed with 2-8 $\mathrm{V}$ pulses with a 0.1-100 ms length, and the transfection rate with this simple device is $36 \%$. This poration rate is greatly improved in another device when another electrode is added, either above the monolayer [39] or as a ring around this primary electrode array [63], to attract the plasmids on the monolayer using dielectrophoresis (DEP) before the poration voltage is applied. The success rate rises from $16 \%$ without any plasmid concentration to $\sim 35 \%$ when an additional electrode is employed (electroporation signal of 12 pulses of $20 \mathrm{~ms}$ and an amplitude of $6 \mathrm{~V}$ ) [63]. Interestingly, in the latter case, only cells on the anodic electrode are transfected, indicating that the negatively charged plasmids only concentrate on that electrode.

\section{Electrode arrays}

Electrode arrays, on which cells are directly grown, have also been reported for poration of monolayers. A main advantage of this approach is that electroporation can be spatially controlled, either by individually addressing specific electrodes for the poration or for patterning the chemicals to be transfected.

For instance, NIH 3T3 fibroblasts have been grown on an $4 \times 4$ or $6 \times 6$ array of individually addressable gold microelectrodes $(100 \mu \mathrm{m} \times 100 \mu \mathrm{m})$ (see figure A.4D) [23-24]. The poration signal is delivered on two adjacent electrodes [23] or between an electrode and a silver wire placed in solution [24], for spatially controlled electroporation. $60 \%$ efficiency is reached with pulses of 10-100 ms length and 2-5 V amplitude. Here, the electrodes are large and covered with 6-12 cells [23-24], but the same approach can be adapted for single cell electroporation, providing the electrodes are small enough to accommodate a single cell [23].

Alternatively, the electrodes can be exploited to pattern the compounds to be injected in the cells. Gold electrodes can selectively be functionalized with a SAM (self-assembled monolayer) on which the material to be inserted in cells is immobilized. Cells are subsequently cultured on the same patterns and selectively porated on the functionalized gold electrodes. This principle is illustrated in the device developed by Fujimoto et al. that contains an array of gold electrodes (1-mm diam. spots) functionalized with siRNAs [62]; 
electroporation is performed using electrical pulses $(10 \mathrm{~ms} ; \sim 12 \mathrm{~V})$ and gene silencing is achieved with a $70 \%$ success rate on cells grown on the electrodes. By attaching various plasmids or siRNAs onto the different spots, parallel transfection experiments are conceivable on a single device.

\section{$3 D$ electrodes}

A final option is to employ three-dimensional electrodes which gives in general, as already discussed earlier, a very uniform field distribution in a larger part of the channel, enhancing the poration probability $[19,35]$.

He et al. describe 3D interdigitated electrodes (12 $\mu \mathrm{m}$ height) $[19,35]$ with a $30-\mu \mathrm{m}$ spacing so that only one cell typically fits in the inter-electrode space. A success rate of approximately $50 \%$ is reported for the uptake of fluorescent dyes with a mild treatment consisting of 1-10 pulses of $2 \mathrm{~ms}$ having an amplitude of 1-6 V. In parallel, pore formation is detected electrically, by measuring the increase in conductance of the cell trapped between the electrodes.

3D electrodes can also be functionalized with the entities to be brought into the cells to enhance the transfection rate. This is described by Miyano et al. who immobilize plasmids on spiked structures fabricated by clustering of a metal alloy (Fe-25Cr-6Ni alloy) (see figure A.4C) [29]. This geometry does not provide a uniform electric field, as is the case of other 3D designs, but their sharp structure results in the creation of hot spots of electric field at their tip. Cells are grown on this spiked functionalized surface and porated in situ; pores are expected to form preferably at the contact areas between the cells and the sharp tips. The resulting transfection rate after electrical treatment ( 1 pulse of $5 \mathrm{~ms}$ and $12 \mathrm{~V}$ ) is not very high $(\sim 30 \%)$, but still greater than that reported for flat plasmid-coated surfaces.

\section{(ii) Discussion}

The main advantage of these monolayer-based devices is their suitability for the in situ electrical treatment of adherent cells. Consequently, these devices mostly combine, in a single platform, different steps of cell culture, electroporation and long-term monitoring of the treatment. For this reason they can be considered as true integrated LOC devices, while their design remains very simple compared to other electroporation systems. The capacity of these devices is quite large, and depends mainly on the surface area of the electrode where cells are cultured. Different designs (planar, interdigitated, arrays or 3D) are conceivable and each of them brings along specific benefits for a better controlled experiment and enhancement of the success rate. For instance, electrode array provides a higher temporal and spatial control on the poration as electrodes can be individually addressable, and this opens the route for individual cell electroporation [23-24]. An additional advantage only found for monolayer-based devices is the possibility to concentrate the molecules to be transfected in close vicinity to the electrodes and the cell monolayer, which promotes cellular uptake upon pore formation. However, in the contrary of (single) cell trapping devices, modeling of the experimentation is difficult as cells are not spherical anymore, but 
stretched on the surface in a non predictable way.

A
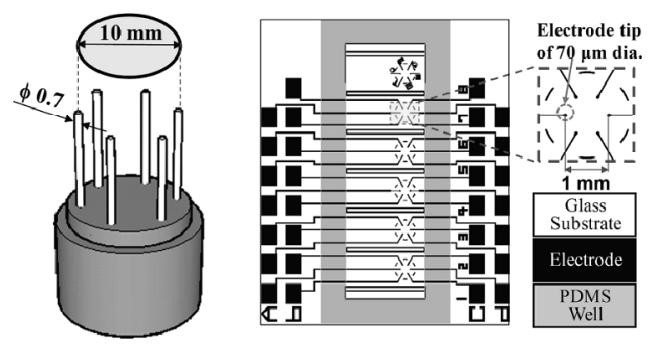

C
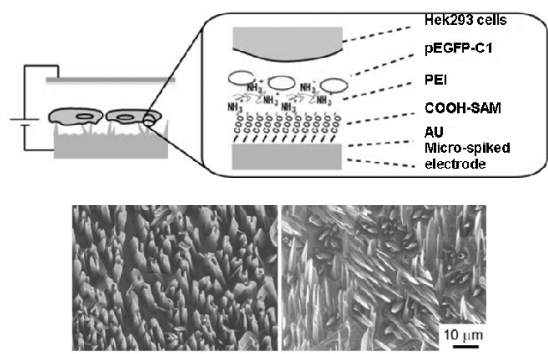

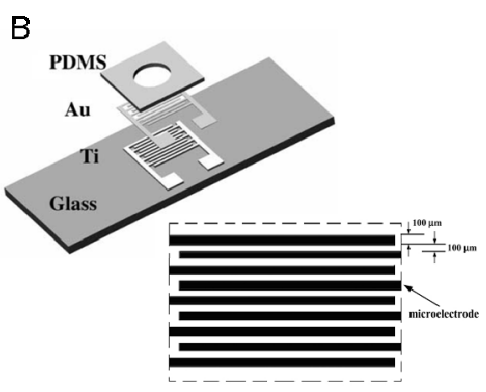

D
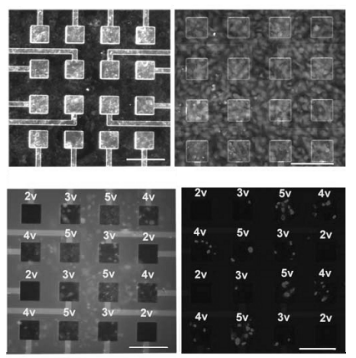

Figure A.4. Monolayer based devices

A) Device fabricated to mimic clinical needle electrode arrays employed for electrochemotherapy. Left: Schematic drawing of a clinical a six-needle circular electrode array for in vivo electroporation. Right: Schematic representation of the planar electrode design employed to mimic this needle array and test is functioning for treating breast cancer cells with the anti-cancer drug bleomycin [17]. B) Illustration of the interdigitated titanium/gold electrode design employed to electroporate cells in the regions of the highest electric fields, i.e. close to the electrodes. The zoom-in shows the layout and dimensions (100 $\mu \mathrm{m}$ wide, $10 \mu \mathrm{m}$ spacing) of the interdigitated electrodes in more detail [39]. C) Top: Depiction of the principle of plasmid-loaded spiked electrodes employed to locally electroporate cells. At the areas where the electrode touches the cells, the field strength is the highest and the membrane becomes porated. Bottom: SEM images of spiked electrode surfaces [29]. D) Top left: A $4 \times 4$ MEA (micro electrode array) employed to electroporate the monolayer of cells grown on it. The electrodes can be stimulated individually, with different signals. Top right: The monolayer of cells with stained nuclei (blue). Bottom left: Fluorescent image after the transfection of Alexa-Fluor-488 conjugated siRNA employing pulses with a different amplitude $(2-5 \mathrm{~V})$ for each electrode. Bottom right: Dead cells (stained red) after electroporation with pulses having different amplitudes $(2-5 \mathrm{~V})$. On the electrodes with the highest applied potential $(5 \mathrm{~V})$ most dead cells are found. The scale bar in all pictures represents $200 \mu \mathrm{m}$ [24].

\section{Conclusion}

Cell electroporation can benefit considerably from its implementation on miniaturized and/or microfluidic devices. The protocol is better controlled, possibly at the single cell level, the success yield greatly enhanced, while the costs and risks associated with the treatment are diminished. These new devices can be classified into three categories depending on the 
way the cells manipulated: trapped in dedicated microstructures, flown as a single-cell file between two electrodes or grown on surface integrating electrodes. Interestingly, these three different types of devices appear to be complementary to each other. (Single) cell trapping devices are the most suited for treating single or rare cells and for following the response of each cell the treatment method individually. Flow-through devices apply better for highthroughput electroporation of large populations of cells and cells can be characterized online before and after the poration process, and the cells can easily be retrieved after their treatment for other applications. The third category of devices is dedicated to adherent cells that can still be treated with the two other types of devices, but not in optimal conditions. There, cells are grown as monolayers, and the devices integrated the different steps of culture, treatment and cell analysis.

The potentials applied to electroporate the cells are in general much lower (2-3 orders of magnitude) compared to the conventional systems. Electroporation signals in microsystems range from $<1 \mathrm{~V}$ to up to $400 \mathrm{~V}$ depending on the system design and the position of the electrodes with respect of the cell sample. This low potential makes the devices not only safer to work with, but also less energy-consuming. Furthermore, the success rate is increased to sometimes reach $100 \%$. Nevertheless, there is still room for improvement, especially in the case of the monolayer-based devices. It is not yet clear why the success rate in these devices is that lower than in other types of miniaturized/microfluidic devices, but the morphology of their cells might play a large role in this.

A large variety of cell lines have been electroporated in such miniaturized and microfluidic devices, and the treatment is followed using different assays. Mostly, optical assays are employed, with fluorescent probes (small organic molecules of fluorescent proteins expressed by the cells after transfection of coding genes). As a proof-of-principle, the introduction of a dye and the transfection of plasmids or siRNA are the most common tests. Sometimes, electroporation is also detected electrically. Not many "clinical applications" are reported yet; the device of Choi [17-18] to study the electrode layout of in vivo electroporators is the only one that mimics a clinically relevant situation.

Flow through or mono-layer based approaches seem to be the most promising approaches for the commercialization of electroporation devices, depending on whether suspension or adherent cell types are used. A requirement for such an apparatus would be that cell properties (e.g. shape, size, membrane capacitance) are firstly characterized on-line to optimize the electrical treatment. Nevertheless, no such systems have been developed so far. A possible reason for this is that the optimization of the electroporation protocol would still be necessary for each cell type, as for the conventional systems, so that, despite the many benefits, the miniaturized devices do not bring additional benefits in that area. Nevertheless, due to the great flexibility these miniaturized systems bring to electroporation experimentation, we see a great future for specialized treatment systems. 


\section{References}

[1] Chen, C. et al. (2006). Med Biol Eng Comput 44 (1-2), pp. 5-14.

[2] Weaver, J.C. \& Chizmadzhev, Y.A. (1996). Bioelectrochem. Bioenerg. 41 (2), pp. 135-160.

[3] Sale, A.J.H. \& Hamilton, W.A. (1967). BBA 148 (3), pp. 781-788.

[4] Sale, A.J.H. \& Hamilton, W.A. (1968). BBA 163 (1), pp. 37-43.

[5] Neumann, E. et al. (1982). EMBO J. 1 (7), pp. 841-845.

[6] Neumann, E. et al., Electroporation and Electrofusion in Cell Biology. Plenum Press: New York, USA, 1989.

[7] Zimmermann, U. et al. (1976). BBA 436 (2), pp. 460-474.

[8] Baker, P.F. \& Knight, D.E. (1978). J PHYSIOL-LONDON 284 (Nov), pp. 30-31.

[9] Gauger, B. \& Bentrup, F.W. (1979). J. Membr. Biol. 48 (3), pp. 249-264.

[10] Zimmermann, U. et al. (1980). Bioelectrochem. Bioenerg. 7 (3), pp. 553-574.

[11] Rols, M.P. (2006). Biochimica Et Biophysica Acta-Biomembranes 1758 (3), pp. 423-428.

[12] Chen, C. et al. (2008). Phys. Med. Biol. 53 (17), pp. 4747-4757.

[13] Gehl, J. (2003). Acta Physiol. Scand. 177 (4), pp. 437-447.

[14] Bao, N. et al. (2008). Electrophoresis 29 (14), pp. 2939-2944.

[15] Bao, N. et al. (2008). Anal. Chem. 80 (20), pp. 7714-7719.

[16] Cho, Y.H. et al., High-Throughput Single-Cell Electroporation Microchip with Three Dimensional Si Microelectrodes for Gene Transfection. In Transduces E Eurosensors, Lyon, France, 2007.

[17] Choi, Y.S. et al. (2009). Anal. Chem. 81 (9), pp. 3517-3522.

[18] Choi, Y.S. et al. (2009). Biomed. Microdevices 11 (1), pp. 151-159.

[19] He, H.Q. et al. (2007). Bioelectrochemistry 70 (2), pp. 363-368.

[20] Huang, Y. \& Rubinsky, B. (2003). Sensor. Actuat. A-Phys 104 (3), pp. 205-212.

[21] Ionescu-Zanetti, C. et al. (2008). Biomed. Microdevices 10 (1), pp. 113-116.

[22] Jain, T. et al. (2009). Lab Chip 9 (24), pp. 3557-3566.

[23] Jain, T. \& Muthuswamy, J. (2007). Biosens. Bioelectron. 22 (6), pp. 863-870.

[24] Jain, T. \& Muthuswamy, J. (2007). Lab Chip 7 (8), pp. 1004-1011.

[25] Khine, M. et al. (2005). Lab Chip 5 (1), pp. 38-43.

[26] Kim, J.A. et al. (2007). Biosens. Bioelectron. 22 (12), pp. 3273-3277.

[27] Klauke, N. et al. (2010). Anal. Chem. 82 (2), pp. 585-592.

[28] MacQueen, L.A. et al. (2008). Bioelectrochemistry 72 (2), pp. 141-148.

[29] Miyano, N. et al. (2008). Lab Chip 8 (7), pp. 1104-1109.

[30] Valero, A. et al. (2008). Lab Chip 8 (1), pp. 62-67.

[31] Valley, J.K. et al. (2009). Lab Chip 9 (12), pp. 1714-1720.

[32] Wang, H.Y. \& Lu, C. (2006). Biotechnol. Bioeng. 95 (6), pp. 1116-1125.

[33] Wang, H.Y. \& Lu, C. (2006). Anal. Chem. 78 (14), pp. 5158-5164.

[34] Wang, H.Y. \& Lu, C. (2008). Biotechnol. Bioeng. 100 (3), pp. 579-586.

[35] He, H.Q. et al. (2008). Bioelectrochemistry 72 (2), pp. 161-168.

[36] Huang, K.S. et al. (2007). Biomed. Microdevices 9 (5), pp. 761-768.

[37] Kim, S.K. et al. (2007). Anal. Chem. 79 (20), pp. 7761-7766.

[38] Lin, Y.C. et al. (2001). Sensor. Actuat. B-Chem 79 (2-3), pp. 137-143.

[39] Lin, Y.C. et al. (2004). Lab Chip 4 (2), pp. 104-108. 
[40] Wang, M.Y. et al. (2009). Anal. Chem. 81 (10), pp. 4060-4067.

[41] Zhan, Y.H. et al. (2009). Anal. Chem. 81 (5), pp. 2027-2031.

[42] Zhu, T. et al. (2010). Biomed. Microdevices 12 (1), pp. 35-40.

[43] Huang, Y. \& Rubinsky, B. (1999). Biomed. Microdevices 2, pp. 145-150.

[44] Huang, Y. \& Rubinsky, B. (2001). Sensor. Actuat. A-Phys 89 (3), pp. 242-249.

[45] Khine, M. et al. (2007). Lab Chip 7 (4), pp. 457-462.

[46] ul Haque, A. et al. (2009). Biomed. Microdevices 11 (6), pp. 1239-1250.

[47] Kim, J.A. et al. (2008). Biosens. Bioelectron. 23 (9), pp. 1353-1360.

[48] Kurosawa, O. et al. (2006). Meas. Sci. Technol. 17 (12), pp. 3127-3133.

[49] Huang, Y. et al. (2003). Sensor. Actuat. A-Phys 105 (1), pp. 31-39.

[50] Le Pioufle, B. et al. (2000). Mat. Sci. Eng. C-Bio S 12 (1-2), pp. 77-81.

[51] Tixier, A. et al. In 1 Annnal Intemational IEEE-EMBS Special Topic Conference on Microtechnologies in Medicine \& Biology 2000, Lyon, France.

[52] James, C.D. et al. (2008). Biosens. Bioelectron. 23 (6), pp. 845-851.

[53] Cho, S.B. \& Thielecke, H. (2007). Biosens. Bioelectron. 22 (8), pp. 1764-1768.

[54] Seo, J. et al. (2004). Appl. Phys. Lett. 84 (11), pp. 1973-1975.

[55] Ionescu-Zanetti, C. et al. (2005). Proc. Natl. Acad. Sci. USA 102 (26), pp. 9112-9117.

[56] Valero, A. (2006). Single Cell Electroporation on Chip. University of Twente, Enschede, ISBN: 90-365-2416-4.

[57] Lu, H. et al. (2005). Lab Chip 5 (1), pp. 23-29.

[58] Sedgwick, H. et al. (2008). Journal of the Royal Society Interface 5, pp. S123-S130.

[59] Lu, K.Y. et al. (2006). Biosens. Bioelectron. 22 (4), pp. 568-574.

[60] Wang, J. et al. (2007). Anal. Chem. 79 (24), pp. 9584-9587.

[61] Ziv, R. et al. (2009). Biomed. Microdevices 11 (1), pp. 95-101.

[62] Fujimoto, H. et al. (2008). Anal. Bioanal. Chem. 392 (7-8), pp. 1309-1316.

[63] Huang, K.S. et al. (2007). Lab Chip 7 (1), pp. 86-92.

[64] Lin, Y.C. et al. (2003). Sensor. Actuat. A-Phys 108 (1-3), pp. 12-19. 


\section{List of abbreviations}

18:1 DOPE-

rhodamine $\mathrm{B}$

AF

AFM

$\alpha-\mathrm{HL}$

ATP

BLM

$\mathrm{Ch}$

CFTR

CE

CG

CNT

DC

DEP

DNA

DOPC

DPhPC

DRIE

dsDNA

EP

ER

FACS

FEP

FIB

FITC

FRAP

GC

GFP

GUV

HTS

IMCD

ITO

L- $\alpha-\mathrm{PC}$

$l_{d}$

$l_{0}$

LOC

LPCVD

LPT
1,2-dioleoyl-sn-glycero-3-phosphoethanolamine-N-

(lissamine rhodamine B sulfonyl)

Amorphous fluoropolymers

Atomic force microscopy

$\alpha$-hemolysin

Adenosine triphosphate

Bilayer lipid membrane

Cholesterol

Cystic fibrosis transmembrane conductance

regulator

Capillary electrophoresis

Coarse grain

Carbon nano-tube

Direct current

Dielectrophoresis

Deoxyribonucleic acid

Dioleoylphosphatidylcholine

1,2-Diphytanoyl-sn-glycero-3-phosphocholine

Deep reactive ion etching

Double-stranded DNA

Electroporation

Endoplasmic reticulum

Fluorescence-Activated Cell Sorting

Fluorinated ethylene propylene copolymer

Focused ion beam

Fluorescein isothiocyanate

Fluorescence recovery after photobleaching

Gas chromatography

Green fluorescent protein

Giant unilammelar vesicle

High throughput screening

Inner medullary collecting duct

Iridium tin oxide

L- $\alpha$-phosphatidylcholine (Heart, Bovine), a mixture of whose predominant species is 16:0/18:2 PC

Liquid disordered

Liquid ordered

Lab-on-a-Chip

Low-pressure chemical vapor deposition

Lipid Plug Thinning 
MEA

MDCK

MDS

MSB

MscL

nAChR

NMR

$\mathrm{OmpF}$

PC

PC

PE

PEG

PDMS

PECVD

PEG

PET

PI

PI

PL

PMMA

POPC

PS

PTFE

PVDC

PVDF

RIE

RNA

SACE

SC

SICM

siRNA

SECM

SEM

SM

So

ssDNA

TCB

$V_{t h}$
Microelectrode array

Madin-Darby Canine Kidney

Molecular dynamics simulations

Micromachined support for bilayers

Mechanosensitive channel of large conductance

nicotinic acetylcholine receptor

Nuclear magnetic resonance

Outer membrane protein $\mathrm{F}$; a nonselective porin

molecules found in the outer membrane of

Escherichia coli

Polycarbonate

Phosphatidylcholine

bovine heart l- $\alpha$-phosphatidylethanolamine

polyethylene glycol

Polydimethylsiloxane

Plasma enhanced chemical vapor deposition

Polyethylene glycol

polyethylene terephthalate

bovine liver l- $\alpha$-phosphatidylinositol

Propidium iodide

Phospholipid

Polymethylmethacrylate

Palmitoyloleoylphosphatidylcholine

porcine brain 1- $\alpha$-phosphatidylserine

Polytetrafluoroethylene

Polyvinylidene chloride

Polyvinylidene fluoride

Reactive ion etching

Ribonucleic acid

spark assisted chemical engraving

Single cell

Scanning ion conductance microscopy

Silencing ribonucleic acid

Scanning electrochemical microscopy

Scanning electron microscopy

Sphingomyelin (Egg, Chicken), a mixture of whose predominant species is $16: 0 \mathrm{SM}$

Solid ordered

Single-stranded DNA

porcine brain total cerebrosides

Threshold voltage for electroporation 


\section{Abstract}

In chapter 1, the aim of the thesis is briefly presented, and a short description content of the chapters is provided.

Chapter 2 introduces the process of electroporation; the technique widely employed to engineer cells and introduced therein foreign materials, with the help of an electrical treatment. In particular, the most accepted theory to explain the process of pore formation process, the so-called transient aqueous pore model, is presented. The limitations of both the technique and conventional set-ups are also discussed: its low success rate, and the need to employ high-voltage sources. Potential approaches to remediate this are finally proposed. Firstly, microfluidic systems can be used instead of conventional mL-capacity set-ups to enhance both the control on the electroporation process and its yield, while employing milder electrical conditions. Alternatively, the knowledge on the process of pore formation can be increased, using for instance planar models of cell membranes. These two approaches of miniaturization and experimentation on membrane models are subsequently applied in the next chapters of this thesis.

The following 5 chapters focus on the second approach and concern the use of planar models of cell membranes, or bilayer lipid membranes (BLMs), to gain a better understanding on the process of pore formation.

Firstly, in chapter 3, a detailed overview is given on BLM experimentation. The advantages of these planar models for experiments on cell membranes are first described. Following this, the different methods reported so far to prepare BLMs mainly in conventional set-ups are described and their advantages and limitations discussed. Next, the potential applications of BLMs are briefly evoked, together with the techniques conventionally employed to characterize them. The second section of this chapter focuses on a novel trend in this field that consists of miniaturizing the experimental set-up and combining it with microfluidics. This evolution in the experimentation platform brings about a great number of advantages which are listed. Finally, a complete overview of miniaturized and microfluidic systems found in the literature is provided, these systems being classified in three main groups depending on their level of miniaturization and "microfluidization": devices where part of the set-up (aperture or fluidic reservoirs) is miniaturized; half microfluidic devices where one fluidic reservoir is replaced by a microfluidic channel; and fully microfluidic devices where two microfluidic channels are employed as reservoirs.

In chapter 4, a first series of experiments using BLM models is reported to study the process of pore formation during electroporation, and in particular, the influence of the membrane composition on this. In this first series of experiments, binary systems of either two phospholipids (glycerolipids), a phospholipid and cholesterol or a phospholipid and proteins are employed. The main building block of the membranes is $\operatorname{DPhPC}(1,2-$ diphytanoyl-sn-glycero-3-phosphocholine) which is chosen for its property to yield very 
stable membranes. To DPhPC, other phospholipids are successively added, L- $\alpha$ phosphatidylethanolamine, L- $\alpha$-phosphatidylinositol and L- $\alpha$-phosphatidylserine. The phospholipid composition of the BLMs in this series of experiments gives slight changes on the membrane stability during the electrical treatment. As previously reported, cholesterol has a more pronounced effect, and this in a concentration-dependent manner. Finally, proteins are modeled with the pore channel $\alpha$-hemolysin, and their insertion quantitatively monitored in the membranes. Up to 6500 proteins can be inserted in the membranes, but electroporation assays are restricted to an amount of 1500, and at this concentration, the presence of $\alpha$-hemolysin has no detectable effect on the electroporation treatment.

Following this, the effect of cholesterol is more deeply investigated in chapter 5 , where ternary systems of a glycerolipid (L- $\alpha$-PC), a sphingolipid (SM) and cholesterol (Ch) are employed in a similar way as in chapter 4 . The motivation for this is to study the influence of possible microdomains found when these three components are mixed, on the membrane properties and its sensitivity to the electrical treatment. Besides, in such ternary mixtures, the three main classes of lipidic components found in cell membranes are represented. Indeed, with such ternary systems, dramatic variations are observed in the membrane resistance to the applied electric field. These variations are successfully correlated to the phases the lipids are in, in the membrane. Interestingly, this last observation enables us to draw a ternary phase diagram for the $\mathrm{L}-\alpha-\mathrm{PC} / \mathrm{SM} / \mathrm{Ch}$ system employed in these experiments.

The last series of experiments using BLMs in the line of getting a better understanding of membrane behavior under an electric field employs even more complex systems, real cell membrane mimics derived from three different cell lines. Particularly, the resistance to electroporation of the apical and basolateral parts of the polarized MDCK cells is examined, as well as the respective behaviors of liver cells and their cancer counterparts. Three main conclusions are found with these experiments. Firstly, these results reveal that a ternary system composed of a glycerolipid, a sphingolipid and cholesterol is not sufficient as a minimal model for all cell membranes. Specifically, glycolipids must be incorporated, especially to recapitulate the outer leaflet of the cell membrane, and the diversity in the glycerolipids found in the inner leaflet can not be represented only by using a simple phospholipid like L- $\alpha$-PC. Secondly, the apical part of MDCK cells and hepatoma cells, which are both supposed to better resist external aggressions, show the same increased resistance to the electric field. This would suggest the existence of a universal mechanism for cells to protect them form perturbations, by modifying their membrane lipidic composition. Lastly, the results obtained on these cell lines could be of interest to derive protocols for in vivo treatment of cells with electroporation (for instance by electrochemotherapy).

The last chapter concerning BLM experimentation examines the miniaturization of the experimental set-up and its implementation in a microfluidic device as this gives numerous advantages compared to a conventional set-up. A microfluidic platform including one BLM experimentation site (50- $\mu \mathrm{m}$ diam. aperture) fabricated in a Teflon thin substrate and placed at the intersection of two orthogonal microchannels is developed and fabricated. Due to the 
closed environment found in such a device, existing BLM preparation techniques had to be modified to produce BLMs inside the system and this is done by successively flushing a lipid solution and measurement buffer. BLM formation can be monitored and characterized either optically using (fluorescence) microscopy or electrically (patch-clamp amplifier). The resulting BLMs are seen to be reproducible and highly stable ( $>7$ hours). Finally, early experimentation on the model membrane protein $\alpha$-hemolysin is successfully performed; this confirms the formation of a bilayer structure and validates the electrical sensitivity of the device.

In the last experimental chapter, the membrane models are abandoned, and a miniaturized device is developed for experimentation on whole cells. This microdevice is particularly developed to electroporate adherent MDCK cells which are grown as monolayers in $200-\mu \mathrm{L}$ wells. Specifically, the cells are grown under polarizing conditions or not, and the influence of their membrane organization on the outcome of the electroporation process is studied, as the results on the membrane models indicated a large effect of membrane polarization on the resistance to the applied electric field. A difference in cell sensitivity to the electroporation voltage is well observed: cells grown under polarizing conditions appear to be more resistant to pore formation. However, the reason for this difference can not with certainty be attributed to their possible polarization as other experimental parameters (e.g., difference in monolayer quality) can affect the outcome of the electrical treatment.

Finally, in the last chapter, the conclusions of this work are summarized. Furthermore, the envisioned fields of applications for both the fundamental and applied studies described in this thesis as well as of the two developed miniaturized devices are discussed. 


\section{Samenvatting}

In dit proefschrift wordt onderzoek naar het elektroporatieproces beschreven. Elektroporatie is een techniek waarbij korte, sterke elektrische pulsen worden gebruikt om poriën te maken in het celmembraan. Deze methode wordt voornamelijk toegepast om bepaalde stoffen in en uit de cel te transporteren. Dit is van groot belang voor veel biotechnologische en medische processen, waarbij de introductie van niet-celeigen materialen, zoals medicijnen, deeltjes of genen nodig is. Hoewel deze techniek al tientallen jaren wordt toegepast, is de succesratio van de behandeling nog steeds vrij laag. In dit proefschrift worden twee methoden gebruikt om het proces te verbeteren; (1) met behulp van fundamenteel onderzoek wordt de invloed van de membraancompositie op het proces van porieformatie onderzocht en (2) door de systemen die gebruikt worden om te elektroporeren te miniaturiseren wordt de controle over het proces vergroot.

In hoofdstuk 1 wordt het doel van dit proefschrift kort uit de doeken gedaan en wordt er een korte beschrijving gegeven van de onderwerpen die in de verschillende hoofdstukken behandeld worden.

Hoofdstuk 2 beschrijft het elektroporatieproces. In het bijzonder wordt aandacht besteed aan de meest gangbare theorie die het porieformatieproces beschrijft, het zogenoemde "transient aqueous pore model". In dit hoofdstuk worden ook de beperkingen van deze techniek besproken: de lage succesratio en het feit dat in traditionele opstellingen een hoogspanningsbron nodig is. Vervolgens worden mogelijke methodes om dit te verbeteren beschreven. Ten eerste kunnen microfluidische systemen worden gebruikt in plaats van de traditionele opstellingen (met een mL-capaciteit) om zowel de controle over het elektroporatieproces als het aantal succesvol behandelde cellen te verhogen, terwijl de aangebrachte spanning om het benodigde elektrische veld te kunnen genereren lager is. Ten tweede kan de kennis over het porieformatieproces worden verhoogd door gebruik te maken van vlakke celmembraanmodellen. Beide aanpakken worden in de volgende hoofdstukken van het proefschrift gebruikt.

De hoofdstukken 3 tot en met 7 beschrijven het gebruik van vlakke celmembraanmodellen, voortaan BLMs (“bilayer lipid membranes”) genoemd, om een beter inzicht te krijgen in het porieformatieproces.

In hoofdstuk 3 wordt eerst een gedetailleerd overzicht gegeven van BLMs. Het voordeel van deze vlakke modellen voor celmembraanexperimenten wordt eerst behandeld. Vervolgens worden de verschillende technieken beschreven om een BLM te maken in voornamelijk traditionele systemen. Ook worden de voor- en nadelen besproken. Daarna worden de toepassingsgebieden van BLMs kort genoemd, samen met technieken om deze te bestuderen. Het tweede deel van het hoofdstuk richt zich op een nieuwe trend: het miniaturiseren van systemen en de combinatie met microfluidica. Deze ontwikkeling brengt een hoop voordelen met zich mee. Het hoofdstuk wordt afgesloten met een overzicht van geminiaturiseerde en microfluidische systemen uit de literatuur. Deze systemen zijn 
ingedeeld in drie klassen, afhankelijk van de mate van miniaturisatie en "microfluidisatie": ontwerpen waar een deel van het systeem (de opening of de fluidische reservoirs) is geminiaturiseerd, ontwerpen waar één van de fluidische reservoirs is vervangen door een microfluidisch kanaal en ontwerpen waarbij beide reservoirs zijn vervangen door microfluidische kanalen.

In hoofdstuk 4 wordt een eerste serie experimenten beschreven waarin het porieformatieproces tijdens elektroporatie wordt bestudeerd, in het bijzonder het effect van de membraancompositie op dit proces. In de eerste series experimenten worden binaire systemen bestaande uit twee fosfolipiden (glycerolipiden), een fosfolipide en cholesterol óf een fosfolipide en een eiwit bestudeerd. Het primaire component van de membranen is DPhPC (1,2-diphytanoyl-sn-glycero-3-phosphocholine) vanwege zijn eigenschap om erg stabiele membranen te vormen. Aan DPhPC worden andere vetten met succes toegevoegd: 1- $\alpha$-phosphatidylethanolamine, $1-\alpha$-phosphatidylinositol and 1- $\alpha$-phosphatidylserine. De fosfolipidecompositie van de BLMs had in deze experimenten maar een kleine invloed op de stabiliteit van het membraan tijdens de elektrische behandeling. Zoals al eerder beschreven, heeft cholesterol een beter zichtbaar effect dat afhankelijk is van de concentratie. Als laatste worden eiwitten gemodelleerd door het eiwitkanaal $\alpha$-hemolysin, en de inserties van dit molecuul in de membranen wordt kwantitatief gemeten. Er kunnen 6500 eiwitten in het membraan worden opgenomen, maar de elektroporatiemetingen zijn begrensd tot 1500 eiwitten. Bij deze concentratie heeft $\alpha$-hemolysin geen meetbaar effect op het elektroporatieproces.

Het effect van cholesterol wordt in meer detail onderzocht in hoofdstuk 5, waarbij gebruik gemaakt wordt van ternaire systemen bestaande uit een glycerolipide (1- $\alpha$-PC), een sphingolipide (SM) en cholesterol (Ch) (de drie hoofdklassen van de vetcomponenten die in celmembranen voorkomen) op dezelfde manier als in hoofdstuk 4. Dit is om het effect van eventuele microdomeinen te bestuderen op de eigenschappen van het membraan en op zijn gevoeligheid voor het aangebrachte elektrische veld. Deze domeinen ontstaan wanneer deze drie membraancomponenten gemengd worden. Voor deze ternaire systemen worden grote verschillen gevonden in de weerstand van het membraan tegen het aangebrachte elektrische veld. Deze variaties worden met succes gecorreleerd met de verschillende fases waarin de vetten zich bevinden. Hierdoor is het mogelijk om een ternair fasediagram op te stellen met behulp van de elektroporatiedata voor het $1-\alpha-\mathrm{PC} / \mathrm{SM} /$ cholesterol systeem dat gebruikt is in deze experimenten.

Om een beter begrip te krijgen van het gedrag van een membraan onder invloed van een elektrisch veld wordt in de laatste serie experimenten met BLMs de complexiteit van het systeem nog een stap verhoogd. Hiervoor wordt gebruik gemaakt van imitaties van echte celmembranen van drie verschillende cellijnen. De weerstand tegen elektroporatie van zowel het apicale en basolaterale deel van MDCK-cellen als van levercellen en hun kankervarianten wordt onderzocht. Uit deze experimenten komen drie hoofdconclusies 
naar voren. Ten eerst, een ternair systeem gemaakt uit een glycerolipid, een sphingolipid en cholesterol is niet afdoende als minimaal membraanmodel voor alle celmembranen. In de modellen moeten glycolipiden worden toegevoegd, zeker om de buitenste helft van het membraan te kunnen nabootsen. Ook kan de verscheidenheid van de glycerolipiden in de binnenste helft niet worden nagebootst door slecht één simpele fosfolipide zoals l- $\alpha$-PC. Ten tweede wordt van het apicaaldeel van de MDCK-cellen en de kankercellen aangenomen dat ze beter bestand zijn tegen agressies van buitenaf. Dit wordt inderdaad bevestigd door hun verhoogde weerstand tegen het aangebrachte elektrische veld. Dit duidt op de aanwezigheid van een universeel afweermechanisme van cellen om zich te beschermen tegen invloeden van buitenaf, door het veranderen van de vetcompositie in hun membraan. Als laatste zijn de resultaten die behaald zijn met deze cellijnen interessant voor het ontwerpen van protocollen voor in vivo behandelingen van cellen met elektroporatie (bijvoorbeeld voor elektrochemotherapie).

Het laatste hoofdstuk over BLMs beschrijft de miniaturisatie van de experimentele opstelling en de implementatie daarvan in een microfluidisch systeem. Een microfluidisch platform is ontworpen en geproduceerd. Dit systeem bestaat uit één locatie voor BLMexperimenten (een opening van $50 \mu \mathrm{m}$ ) gevormd in een dun Teflon substraat, dat op de kruising tussen twee loodrecht op elkaar staande kanalen is geplaatst. Omdat het een gesloten systeem is moeten bestaande BLM formatietechnieken worden aangepast om een BLM te kunnen maken in het systeem. BLMs worden gevormd door achtereenvolgens een vetoplossing en de meetbuffer door de kanalen te spoelen. Het ontstaan van een BLM kan zowel optisch (met behulp van fluorescentie) als elektrisch (met een patch-clampversterker) worden gevolgd en bestudeerd. De BLMs in het systeem zijn reproduceerbaar en erg stabiel (>7 uren). Als laatste worden enkele experimenten met het membraaneiwit $\alpha$-hemolysin uitgevoerd. Die bevestigen zowel de formatie van een bilaag als de elektrische gevoeligheid van het systeem.

In het laatste experimentele hoofdstuk worden de membraanmodellen verlaten en wordt er een geminiaturiseerd systeem ontworpen voor experimenten met cellen. Dit microsysteem is speciaal ontworpen voor het electroporeren van adherante cellen die worden gekweekt als monolagen in $200 \mu \mathrm{L}$ reservoirs. De cellen worden gekweekt onder polariserende en niet-polariserende condities en het effect van de membraanorganisatie op de uitkomst van het elektroporatieproces wordt onderzocht. Er is inderdaad een verschil in gevoeligheid voor de elektroporatiespanning: de cellen die onder de polariserende condities zijn gegroeid zijn beter bestand tegen porieformatie. Helaas kan de reden voor dit verschil niet met zekerheid geweten worden aan de mogelijke polarisatie aangezien andere experimentele parameters (zoals het verschil in de kwaliteit van de monolagen) de uitkomst kunnen beïnvloeden.

In het laatste hoofdstuk worden de conclusies van dit werk samengevat. Daarnaast worden de voorziene toepassingen van zowel de fundamentele als de toegepaste studies uit dit proefschrift beschreven en worden de twee ontwikkelde systemen besproken. 


\section{Dankwoord}

Daar zit je dan plotseling, na vier jaar en drie maanden hard werken, te schrijven aan het laatste gedeelte van je proefschrift: het dankwoord. Dit proefschrift was nooit tot stand gekomen zonder de hulp van vele mensen. Ik wil beginnen met alle mensen te bedanken die ik vergeet te noemen, er zijn zoveel mensen die je ontmoet en die je helpen tijdens je promotie dat ik er vast wel een paar mis, dus bij dezen: Bedankt!

Allereerst wil ik Albert bedanken voor het creëren van deze promotieplek en om mijn wens in vervulling te laten gaan door mij de mogelijk te bieden bij de BIOS groep AIO te worden. Daarnaast zijn jouw ideeën en inbreng tijdens de meetings altijd erg nuttig geweest.

Séverine, zoals je weet was zonder jou dit proefschrift nooit tot stand gekomen. Ontzettend bedankt dat je mijn begeleider hebt willen worden op een project dat toen al anderhalf jaar liep, het is niet makkelijk om ergens midden in te vallen en het je binnen de kortste keren eigen te moeten maken. Je hebt er ontzettend veel energie en tijd ingestoken om mij over de streep te krijgen en ik heb het je vaak niet makkelijk gemaakt. Dat je het toch hebt volgehouden en hulp bent blijven geven, daar ben ik je eeuwig dankbaar voor. Ook heb ik grote bewondering voor je enthousiasme, je creativiteit en je vasthoudendheid om dingen te begrijpen en voor elkaar te krijgen. Je hebt me weten te stimuleren om keer-op-keer het maximale uit mezelf te halen, en dat heeft uiteindelijk dit boek opgeleverd. Daarnaast hebben we ook een hoop lol gehad samen. Aan de gezellige en interessante tripjes naar Île de Berder, San Fransisco en Münster zal ik altijd met veel plezier terugdenken; naast nieuwe dingen leren ook leuke dingen doen (en lekkere cappuccino drinken!).

Daarnaast heb ik ook veel hulp van andere mensen gehad. Een groot deel van de experimenten zijn bij een andere groep van de Universiteit Twente uitgevoerd, de BPE (BioPhysical Engineering) groep. Van de BPE groep wil ik met name Yanina en Martin bedanken voor hun hulp met de BLM opstelling, Tom voor zijn hulp met de confocal microscoop en Vinod voor het toestaan dat ik in het lab heb mogen werken. Remco, zonder de gezellige gesprekken in het chemische lab was het een stuk minder leuke tijd geweest!

Ook vanuit de BIOS groep zijn er veel mensen die hebben bijgedragen aan dit werk. Hans, bedankt dat je altijd snel een passende oplossing wist te maken voor allerlei technische problemen. Johan, ontzettend bedankt voor het meerdere keren laatste-minuut sputteren van de glazen wafers, voor je bijdragen aan het ontwerp van de BLM chip en voor het aandachtig doorlezen en verbeteren van de fabricage procedure in hoofdstuk 7 . Daarnaast wil ik je, samen met Eddy, ook ontzettend bedanken voor het snelle verhuizen en opbouwen van mijn opstelling in het nieuwe lab. Zonder jullie hulp was dat nooit zo soepel verlopen. Jan $\mathrm{N}$, ontzettend bedankt voor jouw hulp met het werkend krijgen van de microscopen en de single-cell elektroporatie opstelling. Henk, bedankt voor je hulp om de instelling van de microscoop te optimaliseren en voor het opbouwen van de "gouden kooi". Paul, al het celwerk was niet tot stand gekomen zonder jouw hulp. Alhoewel het in leven houden van de cellen niet altijd even makkelijk bleek is het ons uiteindelijk toch gelukt! 
Hermine, je deur heeft altijd open gestaan voor al mijn vragen en voor een gezellig gesprek. Bedankt daarvoor!

Naast alle hulp van de bovengenoemde mensen hebben er natuurlijk ook een aantal studenten meegewerkt aan dit project. Brigitte, Julie en Christian, heel erg bedankt voor jullie bijdrage. Verena, ik noem jou apart, aangezien je ontzettend veel hebt bijgedragen aan dit project tijdens je master-opdracht. Heel hoofdstuk 7 beschrijft jouw werk. Ontzettend bedankt voor al de tijd die je erin hebt gestoken en voor je altijd aanwezige glimlach! Ik ben heel blij dat je hebt toegestemd om mijn paranimf te worden!

Werken in de BIOS groep was niet alleen erg interessant en leerzaam, maar ook heel gezellig! Ik heb even zitten tellen, en ik heb een hoop kamergenoten versleten in de afgelopen vier jaar: Arpita, Erik K, Fleur, Ganesh, Georgette, Kevin, Maarten, Rerngchai, Sebastiaan en Svetlana. Bedankt voor alle steun maar toch vooral voor alle gezellige gesprekken! Daarnaast wil ik ook de rest van alle collega's (Ad, Andries, Eddy, Edwin, Egbert, Evelien, Floor, Floris, Henriette, Istvan, Jan E, Justyna, Lingling, Loes, Lonneke, Mathieu, Mingliang, Muhammad, Songyue, Susan, Wouter O, Wouter S, en Yanbo) en oudcollega's (Erik F, Ana, Jurjen, Han, Bianca, Dietrich) van de BIOS groep bedanken voor een supergezellige tijd.

Als laatste wil ik alle vrienden en familie bedanken voor de steun de afgelopen jaren. In het bijzonder Donna voor het toestemmen om paranimf te zijn en voor alle gezellige etentjes met jou en Peter.

Lieve Oma en Saba, ook al woonden jullie duizenden kilometers van elkaar vandaan, toch wil ik jullie samen bedanken voor de interesse en liefde die jullie mij hebben gegeven. Uit alle telefoongesprekken met jou, oma, en de emails met jou, saba, bleek altijd maar weer jullie interesse en trots. Helaas kunnen jullie er beiden niet meer bij zijn, maar ik weet dat jullie hadden zitten stralen van trots.

Lieve broerTJES! (há, nu staat het zwart-op-wit), al zien we elkaar soms minder vaak dan we zouden willen, de gezellige telefoontjes en berichtjes over en weer zijn een hele steun geweest. De weekenden in Groningen, Utrecht, Leiden en Enschede zijn iets om met veel plezier naar terug (en vooruit) te kijken!

Lieve pap en mam, jullie onvoorwaardelijke steun en geloof in mij en mijn kunnen de afgelopen jaren raakt mij diep. Zowel op de momenten waarop ik het niet meer zag zitten als tijdens de hoogtepunten hebben jullie mij altijd met veel enthousiasme gesteund. Ik ben ontzettend dankbaar dat jullie zo hebben meegeleefd de afgelopen jaren; ik kon altijd bellen om mijn hart uit te storten, het gejuich aan de telefoon als er weer eens goed nieuws was, de interesse over waar ik nu precies mee bezig was, noem maar op. Het is erg fijn om te weten dat ik altijd kan bellen voor advies of een gezellig gesprek en dat de deur altijd open staat in Groningen.

Tsja, en dan blijft er nog maar ééntje over van de hele waslijst: Lieve Wouter, het is voor ons allebei niet altijd even makkelijk geweest de afgelopen periode; stress maakt een mens niet gezelliger. Zonder jouw steun, opbeurende woorden en relativerende opmerkingen had 
ik dit nooit gered. Bedankt dat je er altijd was (en bent) wanneer ik je nodig had en dat je me met beide benen op de grond hebt weten te houden. (En ja, ik zal weer wat meer gaan koken hoor! (;) 


\section{List of publications}

\section{Journal articles}

van Uitert, I. et al. (2010). The influence of different membrane components on the electrical stability of bilayer lipid membranes. Biochimica et Biophysica Acta (BBA) - Biomembranes 1798 (1), pp. 21-31. (Chapter 4)

van Uitert, I. et al. (2010). Determination of the electroporation onset of bilayer lipid membranes as a novel approach to establish ternary phase diagrams: example of the 1- $\alpha$ PC/SM/cholesterol system. Soft Matter, 6, pp. 4420-4429. (Chapter 5)

van Uitert, I. et al. (in preparation). Cell membrane heterogeneities affect the outcome of electroporation: implications for in vivo treatment in a clinical setting. (Chapter 6)

Stimberg, V. et al. (in preparation). Bilayer lipid membranes on an integrated microfluidic platform. (Chapter 7)

\section{Lectures}

van Uitert, I. et al., Influence of the membrane composition and the protein density on the breakthrough voltage of BLMs, 19 November 2008, MicroNano Conference 2008, Ede, The Netherlands

van Uitert, I. et al., Towards a Better Understanding of the Process of Electroporation Using Planar Models of Cell Membranes, 21 September 2009, Annual MESA+ meeting, Enschede, The Netherlands

van Uitert, I. et al., Towards a Better Understanding of the Process of Electroporation Using Planar Models of Cell Membranes, 14 January 2010, Mathias Winterhalter's Research Group, Biophysical Chemistry, Jacobs University, Bremen, Germany

van Uitert, I. et al., The Effect of Cholesterol on the Electroporation Process, 21 February 2010, Annual Meeting Biophysical Society 2010, San Fransisco, USA

\section{Conference contributions}

van Uitert, I. et al., Gene transfection protocols in single THP-1 cells using a microfabricated electroporation chip, 13 - 15 June 2006, BioNano Europe Conference 2007, Münster, Germany

van Uitert, I. et al., How to transfect genes into a single THP-1 cell using a microfabricated electroporation chip, 25-31 August 2007, Summerschool: Biosensing with Channels 2007: Channels, Chips and Nanopores, Ile de Berder, France 
van Uitert, I. et al., Transfecting genes into a single THP-1 cell using a microfabricated electroporation chip, 13 - 15 November 2007, Nanotech Montreux conference, Montreux, Swiss

van Uitert, I. et al. Transfecting genes into a single THP-1 cell using a microfabricated electroporation chip, 6 - 7 December 2007, MicroNano Conference 2007, Nijmegen The Netherlands

van Uitert, I. et al., Towards simultaneous electrical and optical investigation of BLMs using a novel microfluidic device, 12 - 16 October 2008, MicroTAS 2008 conference, San Diego, US

van Uitert, I. et al., The presence of ion channels in a membrane disfavors (electro)pore formation in model membranes: an electrical or mechanical contribution?, 15 - 17 June 2010, BioNano Europe Conference 2010, Münster, Germany

van Uitert, I. et al., The individual contributions of membrane constituents on the pore formation process during electroporation, 15 - 17 June 2010, BioNano Europe Conference 2010, Münster, Germany 